\title{
NUCLEAR PHYSICS FOR CULTURAL HERITAGE
}

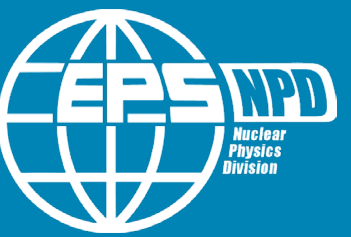

\section{A TOPICAL REVIEW BY}

the Nuclear Physics

Division of the European

Physical Society

\section{EDITED BY}

Anna Macková,

Douglas MacGregor,

Faiçal Azaiez,

Johan Nyberg,

and Eli Piasetzky

INTRODUCTION BY

Walter Kutschera

edpsciences 


\section{NUCLEAR PHYSICS FOR CULTURAL HERITAGE}

P U B L I S H D B Y

Nuclear Physics Division

of the European Physical Society,

October 2016

E D I T E D B Y

Anna Macková, Douglas MacGregor,

Faiçal Azaiez, Johan Nyberg,

and Eli Piasetzky

C O P Y R I G H T

(C)2016 The Authors. This is an open access article under the CC BY-NC-ND

license (https://creativecommons.org/licenses/ by-nc-nd/4.0/).

DOI: 10.1071/978-2-7598-2091-7

ISBN: 978-2-7598-2091-7

C O VER P I C T URE

Early example of an external proton-beam PIXE set-up at the Ion Beam Center, Helmholtz Zentrum, Dresden - Rossendorf, Germany to study the color composition of the panel Die vierzehn Nothelfer by Lucas Cranach the Elder (1472-1553). Figure from C. Neelmeijer, W. Wagner, H.-P. Schramm, Diagnose von Kunstwerken am Teilchenbeschleuniger, Restauro 5 (1995) 326-329.

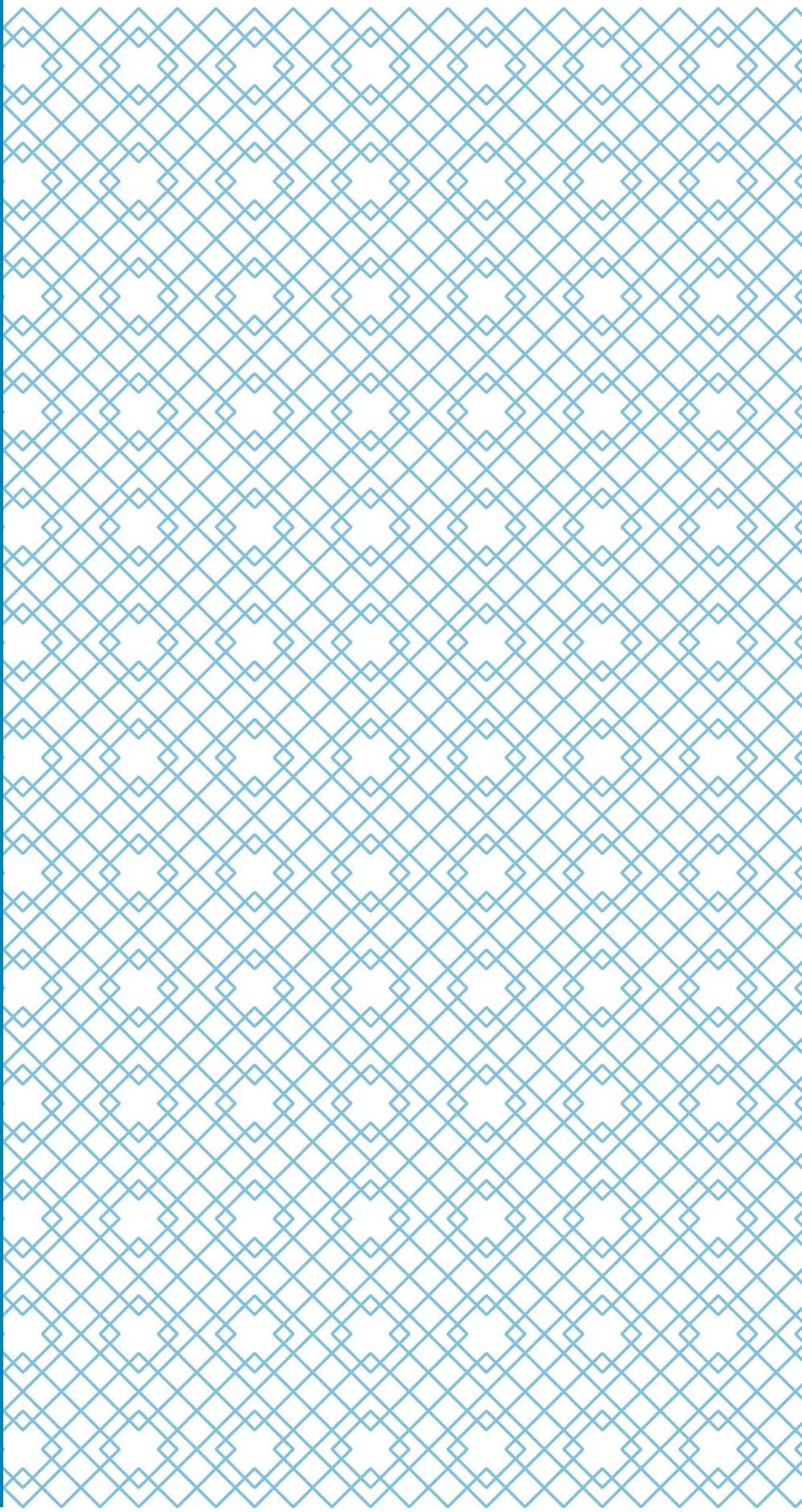




\section{TABLE OF CONTENTS}

FOREWORD

1. IMPORTANCE OF NUCLEAR PHYSICS FOR CULTURAL HERITAGE STUDY AND PRESERVATION

1.1. INVESTIGATION OF CULTURAL HERITAGE OBJECTS

1.2. PRESERVATION OF CULTURAL HERITAGE OBJECTS

1.3. PRESERVE THE OLD, BUT KNOW THE NEW

2.1. BASIC PRINCIPLES OF ION BEAM ANALYSIS (IBA) 


\section{TABLE OF CONTENTS}

5. COMPLEMENTARY METHODS: $\gamma$-BEAM TECHNIQUES, X-RAY FLUORESCENCE (XRF) AND NUCLEAR MAGNETIC RESONANCE (NMR)

5.1. BASIC PRINCIPLES

5.2. INSTRUMENTATION OF COMPLEMENTARY METHODS

5.3. APPLICATIONS OF COMPLEMENTARY METHODS

APPENDIX A: EUROPEAN FACILITIES USING NUCLEAR TECHNIQUES TO STUDY CULTURAL HERITAGE 
I.J. DOUGLAS MACGREGOR,

VICE-CHAIR NUCLEAR PHYSICS DIVISION, EUROPEAN PHYSICAL SOCIETY

\section{FOREWORD}

$\mathrm{N}$ uclear physics applications in medicine and energy are well known and widely reported. See, for example, the recent report "Nuclear Physics for Medicine", published by the European Science Foundation [1] or "Energy for the Future: the Nuclear Option", written by scientists at the European Physical Society (EPS)[2]. Less well known are the many important nuclear and related techniques used for the study, characterisation, assessment and preservation of cultural heritage. There has been enormous progress in this field in recent years and the current review aims to provide the public with a popular and accessible account of this work.

The Nuclear Physics Division of the EPS represents scientists from all branches of nuclear physics across Europe. One of its aims is the dissemination of knowledge about nuclear physics and its applications. Not only is the Division motivated to promote understanding of nuclear issues, it is in a unique position to do this. This review is led by Division board member Anna Macková, Head of the Tandetron Laboratory at the Nuclear Physics Institute, Řež, in the Czech Republic, and the review committee includes four other members of the nuclear physics board interested in this area: Faiçal Azaiez, Johan Nyberg, Eli Piasetzky and myself. To create a truly authoritative account we have invited contributions from leading experts across Europe, and this publication is the combined result of their work. We are grateful to all our contributors for sharing their specialist knowledge with you.

Of course there are previous reviews of work in this field which are aimed at experts. See for instance, "Nuclear Techniques for Cultural Heritage Research", published by the International Atomic Energy Agency [3]. We do not seek to duplicate this work, but rather to present an overview for the more general reader. The review is extensively illustrated with important discoveries and examples from archaeology, pre-history, history, geography, culture, religion and curation, which underline the breadth and importance of this field. The large number of groups and laboratories working in the study and preservation of cultural heritage across Europe (see appendix on European Facilities) indicate the enormous effort and importance attached by society to this activity.

We are grateful to Prof. Walter Kutschera for writing the introduction to our review. His expertise makes him ideally suited to describe the range of techniques, scope of investigation and the degree of innovation which has made this such an important field of study.

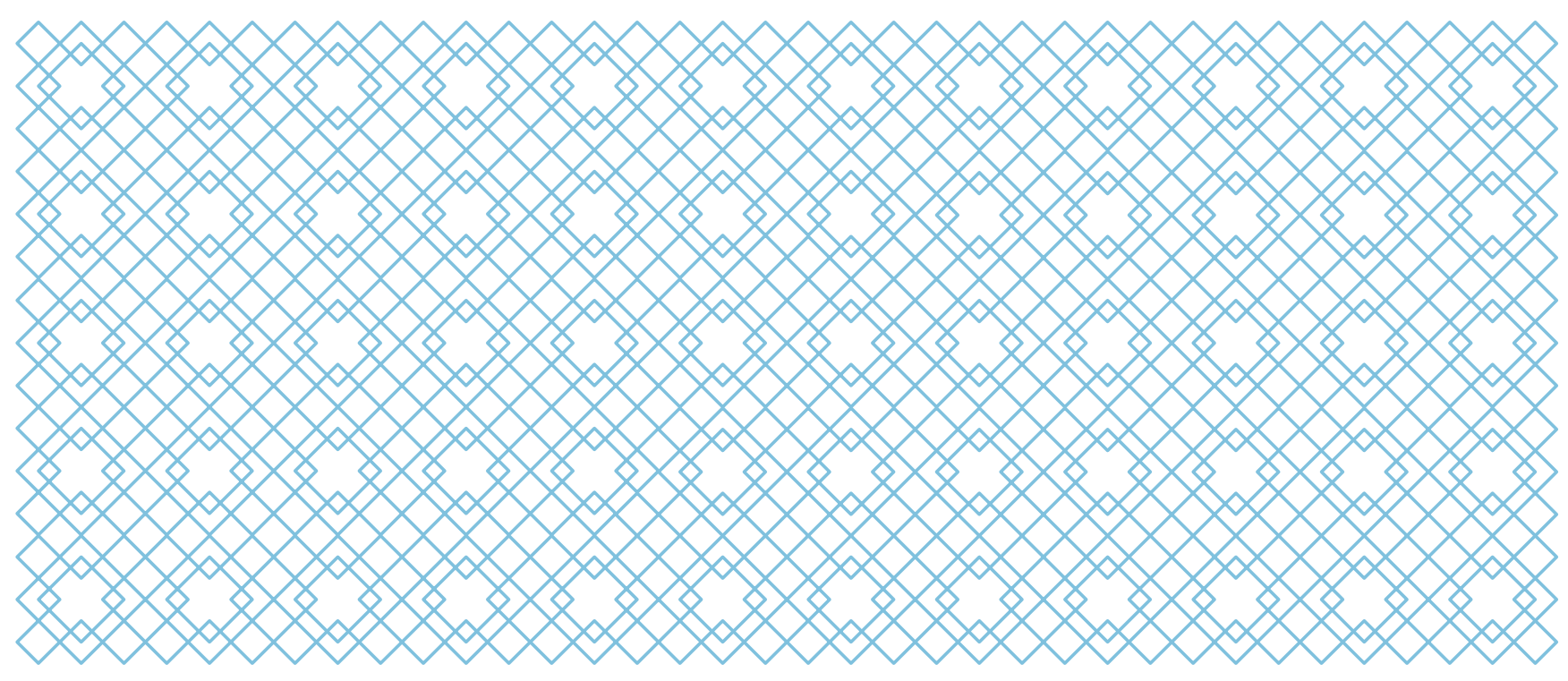




\section{IMPORTANCE OF NUCLEAR PHYSICS FOR CULTURAL HERITAGE STUDY AND PRESERVATION}

T he importance of cultural heritage for mankind was once well expressed by the Austrian artist Friedensreich Hundertwasser (1928-2000) when he said: "If we do not honour our past we lose our future. If we destroy our roots we cannot grow." This statement refers almost directly to the two pillars of this review: Investigation and preservation of our cultural treasures. The various contributions summarised in the current review demonstrate that the methods inherent to nuclear physics are capable of following Hundertwasser's vision. The basic concept is to use nuclear radiations of various kinds (X-rays, $y$-rays, electrons, neutrons and ion beams) to analyse the elemental and/or isotopic composition of an object, or to preserve it by irradiation processes.

\subsection{INVESTIGATION OF CULTURAL HERITAGE OBJECTS}

It is clear that precious cultural heritage objects should remain unaltered after they are exposed to analytical investigation. Therefore non-destructive methods are of crucial importance for investigations. This simply means that the (unavoidable) side-effects of an irradiation must not be noticeable on the object of interest, now or in the future.

This can be primarily achieved by reducing the intensity of the irradiation to very low levels. In order to obtain meaningful analytical information, the low primary irradiation has to be balanced by a correspondingly high detection efficiency of the secondary signal one wants to analyse. Great strides in this direction have been undertaking in recent times, opening up many possibilities to analyse valuable pieces of art.

\subsubsection{ION BEAMS}

Although ion beam analysis developed later than other methods - simply because suitable accelerators only became available in the second half of the $20^{\text {th }}$ century, it is now the most versatile technique for investigating objects of cultural significance. This is due to the flexibility of ion beams, where the beam species (protons, alphas, heavy ions), the energy, the intensity, and the diameter of the beam (sub-millimeter to sub-micron size) can be varied in a suitable way. In addition, the efficiency and resolution of detector systems for X-rays, $y$-rays, and charged particles have greatly improved over the years. An important aspect for ion beam analyses of art objects is the use of an external beam, because often these objects cannot be brought inside the accelerator vacuum system (as an example, see the cover picture of this report). A multitude of different ion beam techniques is now available: NRA (Nuclear Reaction Analysis), PIXE (Proton Induced X-Ray Emission), PIGE (Proton Induced Y-ray Emission), RBS (Rutherford Back-Scattering), ERDA (Elastic Recoil Detection Analysis). All of these are discussed in this review.

\subsubsection{X-RAY AND $Y$-RAY BEAMS}

Since the birth of nuclear physics around 1900, X-rays have been available from the bremsstrahlung radiation emitted by energetic electrons as they pass through materials, and from $X$-rays emitted when an electron vacancy is filled in an atomic orbit (characteristic $X$-rays). The most common method for cultural heritage investigation is XRF (X-ray Fluorescence). Due to its different depth-sensitivity it is complementary to PIXE, and is sometimes combined with it. Portable instruments make XRF a very valuable method for studying objects which cannot be moved to an accelerator. The invention of polycapillary focusing lenses for X-rays led to the development of Micro-XRF, which improved the spatial resolution and thus the versatility of analysing distributions of trace elements. Such developments are being further advanced by utilising the very powerful $X$-rays from electron synchrotrons which are pushing Micro-XRF into the nanometer spatial regime. High-energy $\mathrm{X}$-rays from free-electron laser facilities will likely add another dimension to the quest for ever more detailed X-ray studies of culture heritage objects. 
The European initiative for Extreme Light Infrastructure (ELI) laboratories at ELI-NP in Romania, will provide tunable $\mathrm{y}$-rays from inverse Compton scattering of laser light on a high-energy electron beam. This will allow Nuclear Resonance Fluorescence (NRF) studies of isotope-specific trace element distributions to be performed with unprecedented sensitivity.

\subsubsection{NEUTRON ACTIVATION ANALYSIS (NAA)}

Shortly after the neutron was discovered by Chadwick in 1932, Fermi and others started to convert stable isotopes of many elements into radioactive ones by neutron absorption. In 1936, Hevesy and Levi in Copenhagen realised the analytic power this method had to measure trace elements (particularly REE = Rare Earth Elements). To this day, NAA is used at research reactors, where highintensity neutron sources are available. In combination with high-resolution Ge detectors complex y-spectra from irradiated material can be disentangled, allowing the simultaneous measurement of the concentrations of up to 30 trace elements. Although NAA usually requires bringing the cultural heritage object (or a representative sample of it) to the reactor for neutron irradiation, chemical pre-treatment of the material is not necessary, preserving the original composition of the object. NAA turns out to be particularly useful in the study of trace element distributions in ceramics, helping to determine questions of provenance.

\subsubsection{NUCLEAR MAGNETIC RESONANCE (NMR)}

A frequently applied NMR method in medical diagnosis is called MRI (Magnetic Resonance Imaging), which allows details of soft tissue in humans to be studied by resonantly exciting the nuclear spin of hydrogen in a strong magnetic field. Since the excitation happens with radiofrequency radiation, only non-ionising radiation is used. $A$ big step towards using NMR for cultural heritage was the development of a portable NMR instrument called NMRMOUSE (Mobile Universal Surface Explorer).

\subsubsection{AGE DETERMINATION}

If the absolute age of an object containing organic carbon is of interest, ${ }^{14} \mathrm{C}$ dating is often used. Since this requires taking a small piece of material from the object, it is not a truly non-destructive method. However, counting ${ }^{14} \mathrm{C}$ atoms directly by accelerator mass spectrometry (AMS), rather than counting the infrequent $\beta$-decays (the original method), has increased the detection efficiency of ${ }^{14} \mathrm{C}$ by a factor of about $10^{6}$. This then allows ${ }^{14} \mathrm{C}$ measurements to be performed on very small samples, sometimes as low as a few micrograms of carbon, with negligible effects on the sampled object. The age range extends back to some ten half-lives of ${ }^{14} \mathrm{C}$, i.e. to about 50,000 years. The determination of an absolute age from the measured ${ }^{14} \mathrm{C}$ content requires a calibration curve, which is updated about every five years by an international collaboration committee.

An unusual help to uncover recent art forgery comes from the so-called ${ }^{14} \mathrm{C}$ bombpeak, an abrupt doubling of the atmospheric ${ }^{14} \mathrm{C}$ content around 1960 due to the intense atmospheric nuclear weapons testing period. Finding this ${ }^{14} \mathrm{C}$ excess in an object of supposedly prenuclear origin is an unambiguous proof of forgery.

Inorganic materials, such as ceramics, can be subjected to luminescence dating. Thermo-Luminescence (TL) and more recently Optically Stimulated Luminescence (OSL) are being used, preferably on selected quartz grains from the object to be dated. Here the age determination depends on the production of luminescence centres in a mineral through the radiation dose received from internal and environmental radioactivity. The latter is sometimes difficult to reliably assess for the whole time period to be dated, resulting in a somewhat lower precision than ${ }^{14} \mathrm{C}$ dating. On the other hand, the age range of $\mathrm{TL}$ and OSL is about 300,000 years, considerably longer than that of ${ }^{14} \mathrm{C}$.

\subsection{PRESERVATION OF CULTURAL HERITAGE OBJECTS}

Preservation often requires high intensities of irradiation which may induce changes in the object of interest. One of the main applications is the sterilisation of an object by $\mathrm{Y}$-rays, a method widely used for medical equipment, and sometimes for food as well. The purpose of the irradiation is to kill any bioactivity (e.g. bacteria, fungi, woodworms), which could have adverse effects on the conservation of an object. However, since finite effects are expected on the irradiated objects due to using a high dose of ionising radiation, a careful assessment of these effects must be performed prior to any preservation procedure. Another radiation-assisted procedure for the preservation of objects is consolidation by radiopolymerisation of suitable material added to the object. It is clear that in the various preservation procedures the benefit of a prolonged conservation must be weighed against the unavoidable side effects on the objects one wants to preserve.

\subsection{PRESERVE THE OLD, BUT KNOW THE NEW}

This well-known Chinese proverb can be a guideline for the importance of cultural heritage investigations and preservations - just like Hundertwasser's saying discussed earlier. This review paper demonstrates that we are well on the way to following these guidelines. It is gratifying that nuclear physics, which the public often connects only with the threat from nuclear weapons, radioactivity 
and disasters at nuclear power plants, can contribute in such a significant way to a deeper understanding of our cultural heritage.

There are countless objects of great value stored in museums around the world. The nuclear physics methods described in this review, as well as some other natural science methods, can be used to gain a deeper understanding of their cultural significance. Many of these objects are unique witnesses to the past, and should be investigated with the utmost care. Since one can expect a steady improvement in analytical methods in the future, the value of cultural heritage objects will increase. Therefore, preservation without alteration is a very important goal. In a way, the treatment of material from the moon brought back by the Apollo astronauts in the early 1970 s can be a model. Some of this material is stored away for future generations when improved analyses will be able to extract more information from it than is currently possible.

In general it seems likely that the desire to understand our cultural heritage will grow. This is based on the assumption that from more detailed studies of the past we will simply learn more about us, i.e. the human species which inhabits the Earth now. Besides the methods described in the current review, there are many other ways to enlarge our understanding of cultural heritage, both by methods of sciences and the humanities. Among them a very promising complementary technique is the rapidly evolving field of ancient DNA studies, which will undoubtedly make a major contribution to a better overall understanding of our cultural heritage - and ourselves as well.

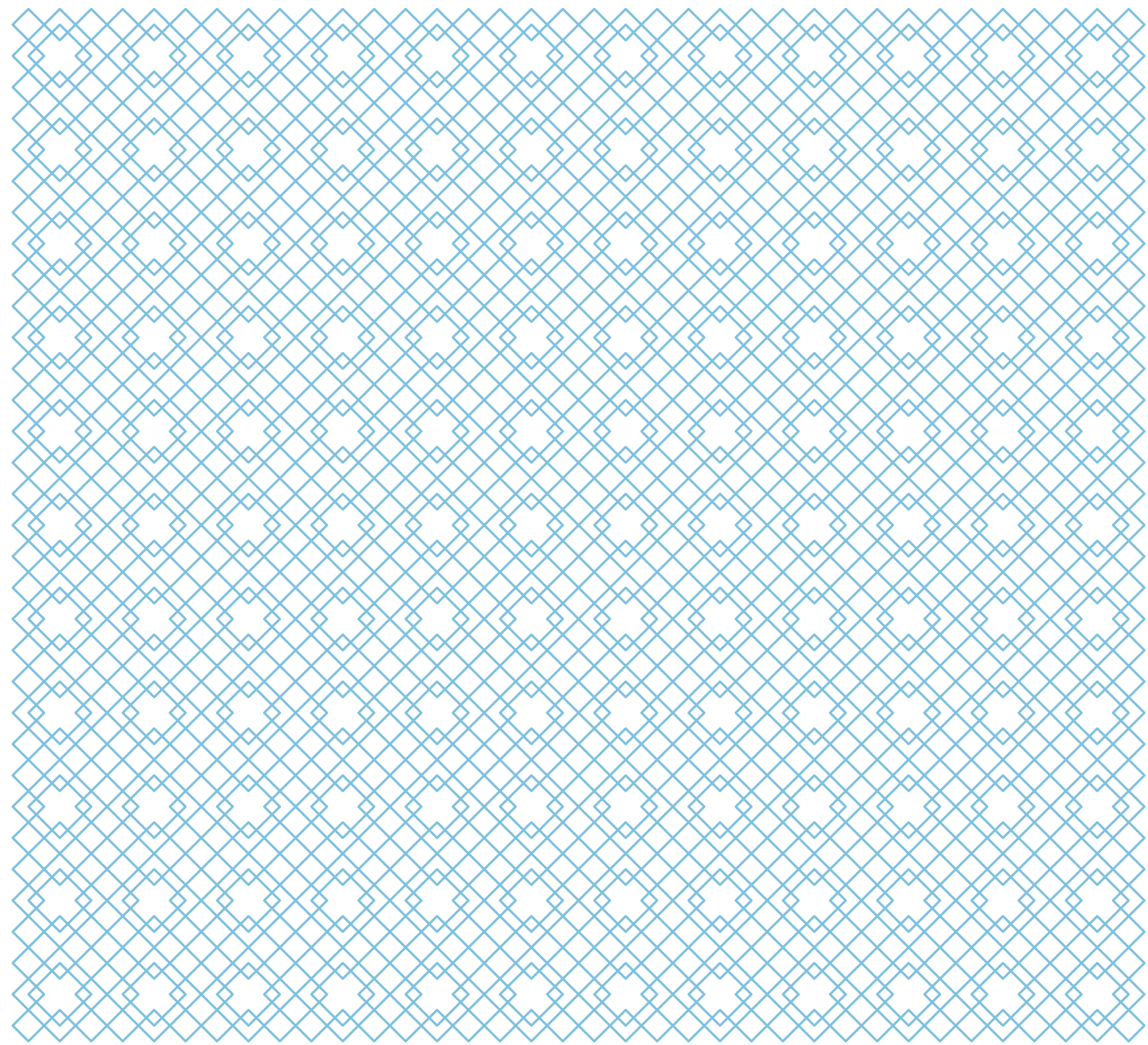




\section{ION BEAM ANALYTICAL METHODS}

T he use of accelerated ions has become an indispensable tool in the analysis of objects and materials in a wide range of scientific and technical studies. Historically, the first nuclear analytical method was Neutron Activation Analysis (NAA), based on principles discovered by Hevesy and Levi in 1936. Later, in the early 1960s, various types of Ion Beam Analyses (IBA) were invented and entered routine use. In the following decades nuclear analytical methods developed and matured, becoming highly valued analytic tools. The most recent development of IBA methods has been strongly related to progress in low energy accelerators, particle, X-ray and gamma-ray detectors and systems for accumulating and analysing experimental data.

\subsection{BASIC PRINCIPLES OF ION BEAM ANALYSIS (IBA)}

\section{NUCLEAR REACTION ANALYSIS (NRA)}

The energy spectrum of charged particles produced in nuclear reactions is measured in NRA. The yield of nuclear reaction products is proportional to the reaction cross section (defined by the probability of a specific interaction) and the density of atoms in the sample. Energy losses by both the incident ions and the reaction products may be used for depth profiling of particular isotopes.

In Resonant Nuclear Reaction Analysis (RNRA) high peak cross sections at resonances give higher sensitivity. Energy loss by the incident ion can be used to determine depth profiles by resonance scanning. Nuclear reaction methods are suitable for identifying a range of isotopes from ${ }^{1} \mathrm{H}$ to ${ }^{32} \mathrm{~S}$. The most frequently used reactions are $(p, a),(d, p)$, and $(d, a)$ in which incident protons, or deuterons, emit a-particles or protons. These reactions provide useful alternative methods for determining isotopes such as ${ }^{2} \mathrm{H},{ }^{12} \mathrm{C}$, and ${ }^{16} \mathrm{O}$, compared with Rutherford Back-Scattering spectrometry (RBS) or Elastic Recoil Detection Analysis (ERDA).

Cross sections of $10-100 \mathrm{mb} \mathrm{sr}^{-1}$ are observed for proton and deuteron-induced reactions on light isotopes, such as $\mathrm{D}, \mathrm{Li}, \mathrm{Be}$, and $\mathrm{B}$. Detection limits of the order of $10 \mu g^{-1}$ or even less are achievable with typical measuring times of the order of tens of minutes. Isotopes up to ${ }^{32} \mathrm{~S}$ can be determined in heavier matrices at $\mathrm{mgg}^{-1}$ levels depending on the maximum beam current that the sample can withstand. The use of glancing measurement geometries or heavy incident ions make possible depth profiling with typical resolutions at the surface of $10-100 \mathrm{~nm}$.

As a result of ion beam irradiation of a material, two types of collision occur: inelastic collisions and elastic collisions.

In inelastic collisions two phases exist. In the first phase particles are emitted (NRA - Nuclear Reaction Analysis). This is followed in the second phase by the emission of $y$-rays (PIGE - Particle Induced Gamma-ray Emission spectroscopy) or X-rays (PIXE - Particle Induced X-ray Emission spectroscopy) $[4,5]$. Nuclear reactions are isotopespecific (the reaction takes place on one particular isotope) with no direct relationship between the mass of the target nucleus and the energy of the emitted particles.

In elastic collisions two main phenomena are taking place: (i) the primary ion beam is backscattered and is used in Rutherford Back-Scattering spectrometry (RBS) and (ii) lighter atomic nuclei can be ejected, recoiling from the heavier projectile ions. This is the principle of Elastic Recoil Detection Analysis (ERDA).

\section{PARTICLE INDUCED GAMMA-RAY EMISSON SPECTROSCOPY (PIGE)}

PIGE or PIGME (particle-induced gamma-ray emission) is a versatile non-destructive analytical and depth profiling technique based on the $(p, \gamma)$ reaction [6-9]. The $y$-ray peaks are generally well isolated and the energy is high enough that no absorption correction is necessary.

The energies and intensities of the $\gamma$-ray lines indicate which elements are present and their respective amounts. 
For protons with energies from 1 to $3 \mathrm{MeV}$, the best sensitivities are found for $\mathrm{Li}, \mathrm{B}, \mathrm{F}, \mathrm{Na}$, and Al. These elements can be determined simultaneously in many cases. Concentrations of $\mathrm{F}$ and $\mathrm{Na}$ can be obtained with uncertainties below $1 \%$, in only a few minutes.

At proton energies above $3 \mathrm{MeV}$, the $\mathrm{Y}$-ray emission from medium and heavy elements begins to compete with that from light elements. The highest cross sections are for light isotopes $(A<30)$, which can be determined with a sensitivity of $1 \mu^{-1}$ or less.

\section{PARTICLE INDUCED X-RAY EMISSION SPECTROSCOPY (PIXE)}

PIXE uses X-ray emission for elemental analysis [4, 10-13]. Samples are irradiated by an ion beam from an accelerator and characteristic $X$-rays are then detected by $\mathrm{Si}(\mathrm{Li})$ or HPGe detectors. Ions, or protons, with energies of a few MeV ionise atoms in the sample and induce the emission of characteristic X-rays. PIXE is not a true nuclear technique, as the ionization of atoms by the ion beam and the subsequent emission of characteristic $\mathrm{X}$-rays are purely atomic processes. The energy of the emitted X-rays is a monotonously increasing function of atomic number (Moseley's law). Hence, the energy of a peak in the X-ray spectrum is specific to a particular element and its intensity is proportional to the element's concentration.

As a result of its short measurement time, PIXE is the preferred method for the analysis of thin samples, e.g. from air filters, or for the automated analysis of large numbers of geological or archaeological samples. The concentrations of up to about 20 elements may be determined simultaneously. The low absolute detection limit, and good sensitivity, for elements such as $\mathrm{S}, \mathrm{P}, \mathrm{Cl}, \mathrm{K}, \mathrm{Ca}$ and $\mathrm{Fe}$ make PIXE of great importance in biological, archaeological and medical applications.

The X-ray yield depends on the number of atoms in the sample, the ionisation cross section, the intensity of the ion beam, the energy-dependent detection efficiency of the semiconductor detectors used, the composition of the sample and several other additional factors. The determination of an absolute concentration of an element in an unknown matrix is a complex problem. In practice, the evaluation of sample composition involves the use of standards and reference materials to determine the calibration curve of a particular measurement set-up. Depending on the sample type and measuring apparatus, the concentration of elements with $Z>5$ can be determined with sensitivities of $0.1-1 \mu \mathrm{gg}^{-1}$. PIXE has very low detection limits from $10^{-8}-10^{-10} \mathrm{~g}$ in standard practice. This method is not used for elemental depth profiling, because of its low depth resolution.

The major advantage of PIXE's use of ions is a reduction in the background in comparison to that obtained when electrons are used as a probe (electron microprobe induced X-ray emission, EDX).

Differential PIXE (d-PIXE) is based on sequential measurements in the same locations so that protons reach different target depths. This is achieved either by variation of the incident proton angle or by variation of the proton energy. In either case, the strongest X-ray signal comes from the target surface, which largely screens out the contributions from inner layers. Sensitive numerical methods are then required to filter out these minute contributions. The results of the de-convolution procedure are concentration profiles, which can reach up to $10 \mu \mathrm{m}$ below the target surface.

\section{RUTHERFORD BACK-SCATTERING SPECTROSCOPY (RBS)}

In elastic collisions two main phenomena provide analytical information: (i) the energy transfer and (ii) the kinematics of elastic collisions between atomic nuclei and ions.

RBS is the most commonly used non-destructive nuclear method for elemental depth analysis of structures in the nanometer to micrometer thickness range $[4,5,13-$ 17]. Typical objects are thin surface films. The method is based on measurements of the energy spectra of several MeV ions (protons, singly charged helium $\mathrm{He}^{+}$, or heavier ions) elastically scattered from solid samples. The samples are irradiated in an evacuated target chamber and the scattered particles are detected by semiconductor detectors. The energy spectra are evaluated using standard codes and information on the sample composition and the depth distribution of particular components is obtained. As a consequence of the scattering kinematics, the energy of the scattered particles increases monotonically as a function of the element mass.

The scattering cross section is proportional to the sample element atomic number squared. Thus the technique is particularly sensitive to heavier elements. The quantity of a particular element in the target is proportional to the number of scattered particles. The incident and scattered particles penetrating through the sample material lose energy progressively and the measured energy loss can be transformed into a depth using the known particle stopping powers in the sample material. 

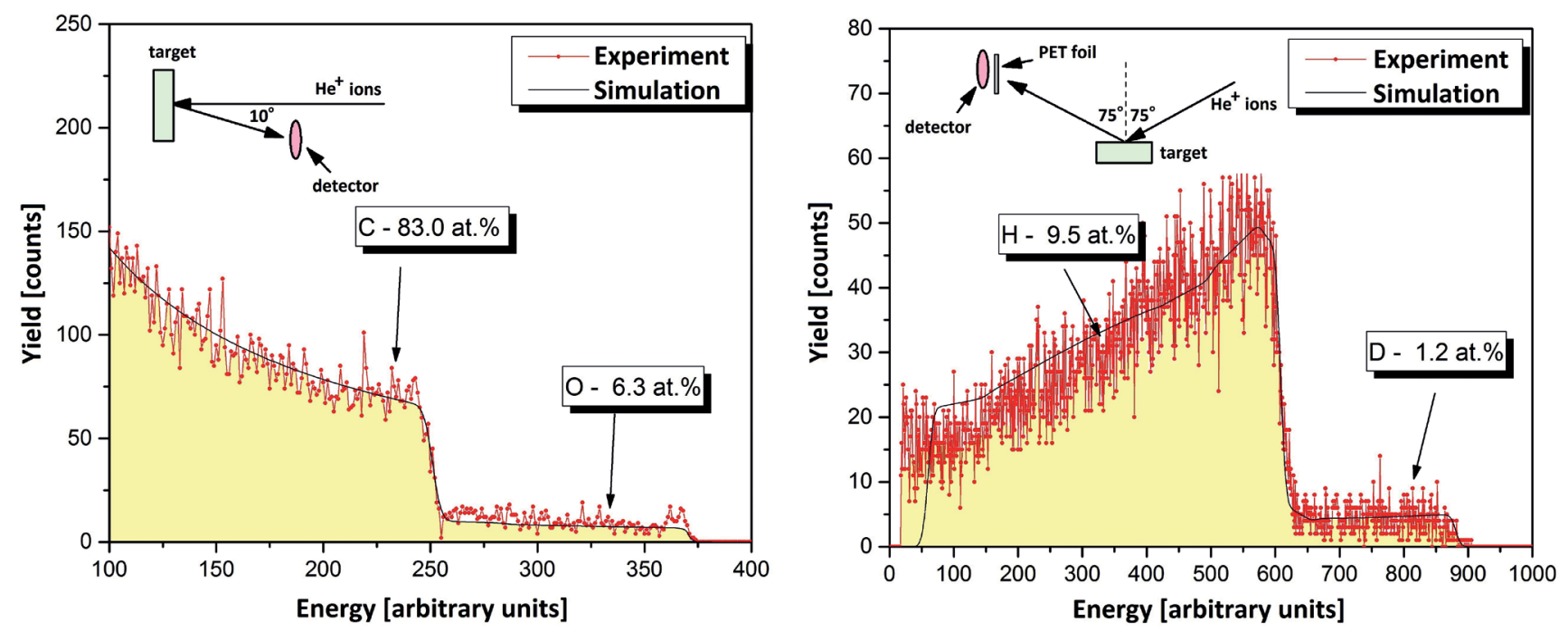

Figure 2.1: Complementary Rutherford Back-Scattering (RBS) and Elastic Recoil Detection Analysis (ERDA) analyses of deuteriumdoped graphene based structures for depth profiling of heavy impurities, compositional studies and light dopants [18].

This makes it possible to determine the depth distribution of particular elements with a resolution as low as $10 \mathrm{~nm}$. The sensitivity of RBS for the detection of trace impurities in bulk samples depends strongly on the sample composition and the experimental conditions. For heavy elements, in a light substrate, detection limits of about 0.01 atomic percent (at. \%) can be achieved.

The major strengths of RBS are its relative simplicity, its non-destructive nature and the possibility of determining the detailed structure of samples. Figure 2.1 shows the combined analysis of light elements in graphene based structures using RBS and ERDA, which is described later in this section.

\section{RBS-CHANNELLING (RBS/C) SPECTROMETRY}

RBS-channelling spectrometry is a method of investigating adventitious atoms located in the interstitial space of single crystals [19-21]. A beam of energetic ions is steered into open spaces (channels) between closepacked rows or planes of atoms in a crystal. Figure 2.2 shows the image of a single crystal rotated in a $2 \mathrm{MeV}$ $\mathrm{He}^{+}$beam.

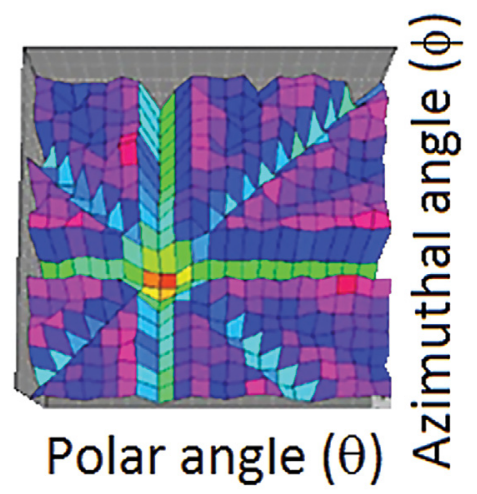

Figure 2.2: Image showing the RBS back-scattering yield of $2 \mathrm{MeV} \mathrm{He}^{+}$ions, from a single crystal as a function of the polar and azimuthal angles the crystal is rotated through.
The yield depends on the crystal orientation with respect to the ion beam and changes as the polar and azimuthal angles $(\theta, \varphi)$ between the crystal and the incident beam are varied. The observed intensities are reduced at angles corresponding to channelling between crystalline planes. The most prominent valley corresponds to an axial channel where the most ions are steered into the crystal and the back-scattered yield is very small. This direction corresponds to the orientation of the main crystallographic axis in the crystal.

The energy transfers or kinematics in elastic collisions between ions and atomic nuclei can give information about the composition and structure of the sample. The number of scattered particles measured by a detector can be converted to the concentration of a particular element in the target. The incident particle energy losses are much lower in the channelling regime compared to random incidence.

The energy spectrum of backscattered particles from an aligned crystal is dramatically different from that of non-aligned, randomly placed sample. In the aligned spectrum, the scattering yield from the bulk of the solid is reduced by around two orders of magnitude and a surface peak occurs. The presence of defects can significantly enhance the de-channelling yield comparing to a perfect crystal.

The backscattered yield from interstitial atoms does not exhibit the same decrease as that of the host crystal and can be used either for evaluation of the impurity position in a host crystal lattice or for the study of the displacement of host atoms from their lattice sites.

The major strength of RBS/C is an ability to determine the position of impurity atoms in a host crystal lattice. $\mathrm{RBS} / \mathrm{C}$ is usually employed for the analysis of samples of known composition with the focus on impurity atoms or the number of defects. 


\section{ELASTIC RECOIL DETECTION ANALYSIS (ERDA)}

ERDA is one of the most useful ion beam analysis techniques for depth profiling of light elements [22-26]. A beam of energetic ions is directed towards the sample. When the incident ion has a heavier mass than the sample atoms, a light target atom may be knocked out and detected in a forward geometry using a semiconductor detector (see inset of Figure 2.1). Atoms recoiling from the surface appear at different energies depending on their mass and measuring arrangement.

The sensitivity of ERDA depends on the experimental arrangement and the system dependent background level. Typically 0.1 at. \% of ${ }^{1} \mathrm{H}$ is observable and from 0.1 to 1 at. \% of heavier atoms. Simple ERDA, using charged particle detectors with a stopping foil in front, has a depth resolution of typically $20-60 \mathrm{~nm}$.

The stopping foil has to be thick enough to absorb primary ions elastically scattered from the sample. With higher mass projectiles, heavier elements such as $\mathrm{N}, \mathrm{O}$, and $\mathrm{F}$ can also be analysed by the simple ERDA technique. Absolute measurements of light atom content by ERDA are best achieved by using standards.

The arrangement with the stopping foil is not suitable for analyses of heavier elements using heavy projectiles; in this case heavy ion elastic recoil detection analysis (HIERDA) using ionisation chamber detectors and energy detectors, or time-of-flight techniques (TOF-ERDA), could be used to separate the masses and energies of the recoiling particles.

\subsection{INSTRUMENTATION OF IBA}

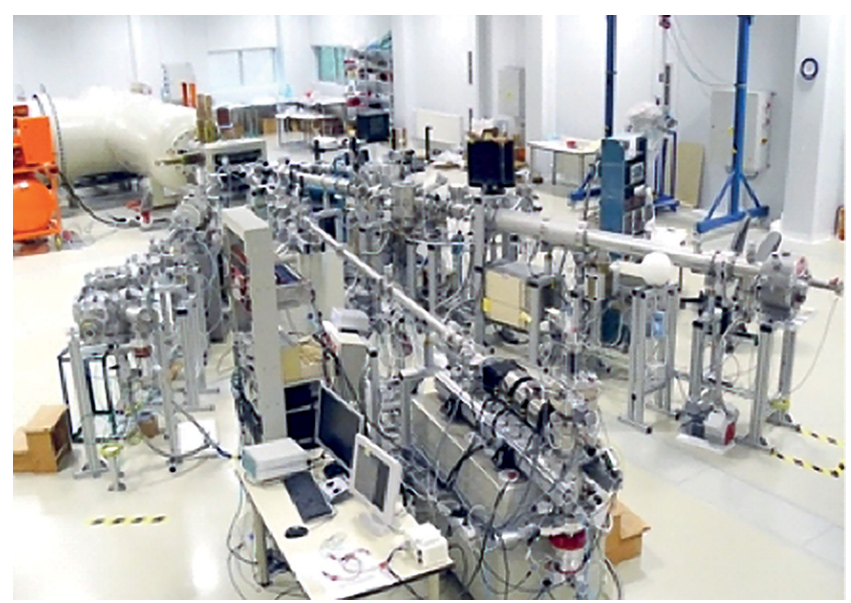

Figure 2.3: Tandetron accelerator with ion beam lines, vacuum chambers and detectors arrangement at the Center of Accelerators and Nuclear Analytical Methods (NPI CAS),

Czech Republic, used for various nuclear analytical methods.

Standard equipment for IBA analysis comprises an electrostatic accelerator (see Figure 2.3), providing the ions (protons, deuterons, He and heavier ions) with energies from $0.5-50 \mathrm{MeV}$, with associated ion beam-lines and vacuum target chambers in which the samples under study are irradiated. The samples are mounted, several per load, on the table of a goniometer for precise positioning and orientation of the samples with respect to the incoming ion beam.

The products of ions interaction with sample atoms are registered by semi-conductor detectors with associated electronic devices for processing detector signals and data acquisition. An important part of the equipment is a device monitoring the beam intensity; Faraday cups, rotating vanes intersecting the beam or a thin wire mesh inserted in the beam are common techniques.

In general, the ion beam hits the sample at normal incidence. If the ion energy used is equal to the resonant energy in the RNRA method, the resonance reaction takes place on nuclei located at the surface. If the beam energy is higher than the resonant energy, the resonance occurs at depth, because of energy losses of the initial ions. By measuring the yield for a constant accumulated charge and varying the beam energy in small steps, the yield as a function of ion beam energy can be interpreted as the quantity of the element at various depths. That is, it provides the concentration depth profile. Incident ion energies from 0.5 to $2 \mathrm{MeV}$ are most useful for minimising interference from reactions on heavy isotopes.

PIGE is mostly based on ( $p, y),\left(p, p^{\prime} \gamma\right)$, and ( $\left.p, a y\right)$ nuclear reactions induced by $\mathrm{MeV}$ protons where nuclear $\mathrm{y}$-rays are produced. In most cases, high purity germanium (HPGe) or scintillation detectors with multichannel acquisition systems are used for detection of $y$-rays. The lower the incident ion energy, the fewer resonances are involved in ion- $\gamma$ reactions and non-uniform angular distributions are more likely to be observed.

PIPS, or surface barrier detectors, are primarily used for detecting scattered ions in RBS and ERDA methods. A channelling RBS experiment requires a source of collimated high-energy ions from an accelerator, a detector for scattered particles (the same as for RBS), and an accurate crystal manipulator (goniometer). The goniometer is a crucial part of the equipment which allows the crystal axes to be aligned with the collimated particle beam.

ERDA relies on the ability to discriminate between forward scattered incident ions and recoiling light atoms. The typical experimental arrangement is a Mylar foil placed in front of the detector to block out the scattered incident ions but allow the lighter recoil atoms, which suffer considerably less energy loss, to pass through to the detector. Note that a $10 \mu \mathrm{m}$ Mylar foil completely stops $2.6 \mathrm{MeV} \mathrm{He}^{+}$ions, but $\mathrm{MeV}$ recoil protons pass through with low-energy losses. Thus, $\mathrm{He}^{+}$ions are used for hydrogen profiling.

Heavy ion-ERDA (HIERDA) is able to analyse light and medium elements. Typically heavier ions such as $\mathrm{Cl}^{\text {nt }}$ or $\mathrm{I}^{\mathrm{n}+}$ are used, with energies of tens of MeV. HIERDA needs an appropriate detection technique to distinguish the large 
numbers of different particles that recoil simultaneously. The technique uses either the simultaneous measurement of the energies and velocities of the detected particles (TOF measurement) to separate the mass of recoils, or a gas-filled ionisation chamber for mass separation. The velocities in TOF measurements are determined by measuring the elapsed time between the detection of a particle in two sequential detectors placed a fixed distance apart. Gas filled detector measurements determine both the total energy and the energy loss of the recoiling particles. The signals from recoil elements, which overlap on a simple energy spectrum, are separated by their different energy loss rates.

\section{SPECIAL INSTRUMENTAL ARRANGEMENTS}

In ion microprobe analysis, the samples are irradiated with an ion beam focused to a spot about $1 \mu \mathrm{m}$ in diameter and standard IBA techniques (PIXE, RBS) are used for the characterisation of the part of the sample which is irradiated. By scanning the beam across the surface of the sample a 3D distribution of elements can, in principle, be determined with a nm depth resolution and a lateral resolution limited only by the size of the beam spot. For this purpose the signals from the detectors are recorded as a function of the current position of the beam spot. See Figures 2.4 and 2.5 .

A fully equipped proton microprobe (PMP) chamber should include microscopes for transmission and reflective viewing of the specimen, a $\mathrm{Si}(\mathrm{Li})$ detector for detection of X-rays, surface barrier detectors for backward and forward collisions, and a detector for y-rays. Charged particle beams are focused by means of magnetic or electrostatic lenses. The achievement of good spatial resolution requires a good ion optics design, high precision in fabrication, careful alignment, and elimination of sources of interference.

When the ion passes through a thin specimen, the beam transmitted in the forward direction includes some particles that scattered elastically off atomic nuclei, or lost energy as a result of interaction with electrons, as well as those particles that were not scattered. An image formed with this forward transmitted beam is referred to as a bright field image.

In order to measure the distribution of elements along a line, or map the elemental distribution over an area, the focused beam spot must be scanned and the detector signal recorded as a function of the displacement of the beam from its normal position. When a beam of ions scans an area of a specimen, the emitted radiation carries

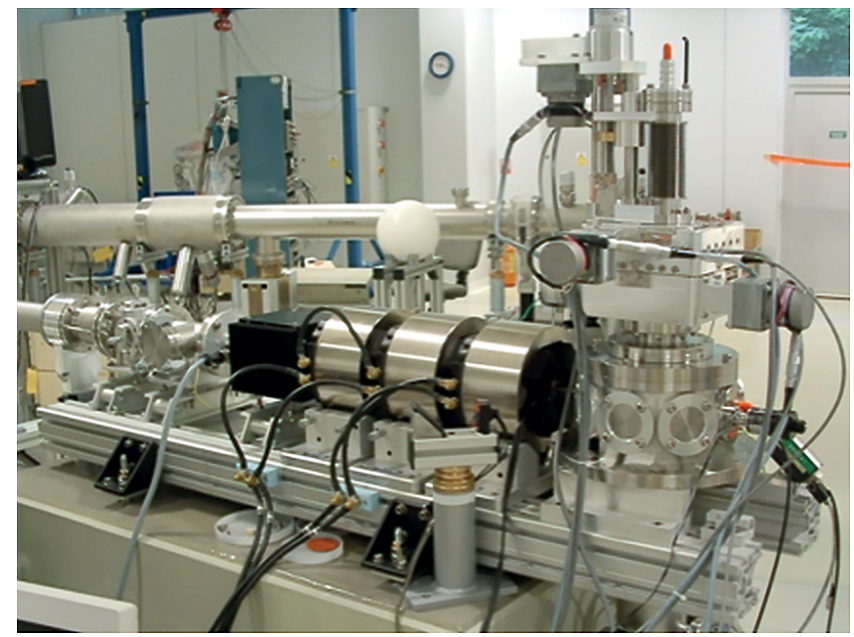

Figure 2.4: Microbeam arrangement at the Center of Accelerators and Nuclear Analytical Methods (NPI CAS), Czech Republic, showing the vacuum chamber for the specimen on the right and a triplet of magnetic quadrupole lenses for focusing the beam to sizes of a few micrometers.

information in 3 degrees of freedom - the two scanning dimensions and the energy. Scanning ion microprobe (SIMP) and scanning proton microprobe are very useful techniques for in situ element or isotope distribution analysis. See Figure 2.5.

With protons or heavy ions, the mean free path between ionising events is generally much shorter than the specimen thickness and multiple inelastic collisions occur. The energy-loss spectrum becomes a measure of specimen thickness rather than elemental content. In proton microprobe (PMP), with a typical energy of $3 \mathrm{MeV}$, the proton range is some tens of micrometers and the mean free path between inelastic collisions is under $100 \mathrm{~nm}$.

In bright-field transmission imaging, the transmitted beam runs directly into a detector and the beam current is restricted to about $10^{4}$ particles $\mathrm{s}^{-1}$. PMP gives a spatial resolution for microanalysis of about $1 \mu \mathrm{m}$, with 100 pA beams of protons or a-particles. Some effects must be taken into account, such as the charging of insulating components and the removal of some components by sputtering, which prevents repeated investigations. Image contrast may also arise from chemical or topographic rather than isotopic differences.

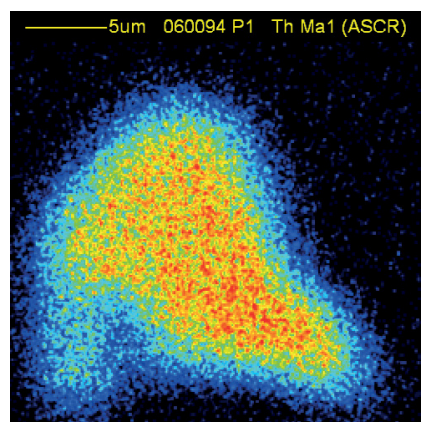

Figure 2.5: 2D microbeam mapping of the elemental composition of an inclusion in a granitic rock, obtained by scanning the microbeam. The colour indicates the concentration of the element studied with the highest concentration depicted by the red and yellow colours. 


\section{EXTERNAL BEAMS}

In practice materials or artefacts are often obtained which cannot be placed in a vacuum chamber because of their large size or because of volatile components. Such samples can be analysed using an external ion beam, extracted from an evacuated beam line into air through a thin window. This typically reduces the beam energy by 20-200 keV.

The window materials are either thin metal foils, such as aluminium or tungsten, or strong plastic materials like kapton or $\mathrm{Si}_{3} \mathrm{~N}_{4}$, which is now widely used. This material typically has a very low thickness, about $0.1 \mu \mathrm{m}$, to minimise the energy loss and angular straggling of the external beam. In a standard arrangement the beam spot at the target is a millimetre or less in diameter if the beam is shaped by slits, but may be as low as 10 to $30 \mu \mathrm{m}$ if the beam is focused using magnetic optics.

Targets are normally mounted on a computer-controlled $x-y-z$ placeholder. Practically all arrangements now allow the scanning mode of measurement that produces concentration maps. The target is encircled by an array of detectors: normally at least two X-ray detectors are used: a thin window detector for soft $X$-rays and a detector with a large solid angle, but equipped with an additional absorber, for hard X-rays. The target region may be flushed with helium to reduce $\mathrm{X}$-ray absorption and X-ray background arising from interaction of the ion beam with $\mathrm{Ar}$ in the air.

In recent measurement configurations, X-ray $\mathrm{Si}(\mathrm{Li})$ detectors are replaced by arrays of SDD (silicon-drift detector) diodes and induced $\mathrm{y}$-rays are measured by HPGe or scintillating ( $\mathrm{Nal}-\mathrm{TI})$ detectors. Analyses can also be carried out at external beam RBS and ERDA and, for this purpose, flushing with helium is necessary. Some other types of spectroscopy are installed at some facilities, such as ion-induced optical luminescence where optical spectra can reveal information on chemical bonding.

\subsection{APPLICATIONS OF IBA}

The application of atomic and nuclear techniques to the study of archaeological objects gives the historian or the archaeologist materials information that helps understanding life during ancient times. This knowledge is necessary to test the authenticity and provenance of artefacts and to prepare and carry out restorations. These objectives are common to the large community of people working in archaeometry, i.e. the application of science to art and archaeology. In the case of investigating metallic artefacts, the domain is called archaeometallurgy. For these research activities a multidisciplinary community of action is essential.
IBA techniques are based on relatively simple, well-known physical processes and the extraction of the required information from the measured spectra is reasonably straight-forward. IBA measurements and data evaluation can also be performed in a relatively short time. With a single experimental facility, simultaneous analyses using various IBA methods can be accomplished and more complex analytical information easily obtained. This work brings together physicists, chemists, archaeologists, numismatists, historians, geologists and conservators from different laboratories, institutes and museums.

\subsubsection{METALS}

Archaeological metals are efficiently studied by ion-beam methods; however, one has to consider that the range of $\mathrm{MeV}$ particles in metal is typically $10-30 \mu \mathrm{m}$, which renders the techniques such as PIXE surface-sensitive only. As archaeological metals are normally covered by oxides up to a millimeter thick, these have to be removed in order to get information on the bulk composition. Surface polishing is often not permitted by museum curators, though they may consent to it if the collected analytical information is valuable. Such corrosion problems are virtually absent with noble metals, though one has to be aware that some metal enrichment at the surface may occur due to selective oxidation or leaching of less noble metals.

As an illustrative application of metal analysis we show a study of Roman brass [27]. Brass, an alloy of copper and zinc, appeared relatively late among the set of historical alloys. The problem of alloying brass is a low evaporation temperature of zinc; zinc may evaporate before the copper melts. Brass was first produced only occasionally by melting together copper and zinc ores, until the invention of the so-called cementation technique. This procedure relies on the simultaneous reduction of zinc ores in the presence of copper in a sealed container which enables simultaneous diffusion of zinc into copper. The maximum percentage of zinc in cementation-produced brass is $28 \%$ [28]. Brass coins appeared among the Hellenistic Greeks around $100 \mathrm{BC}$.

Polished brass with its gold-like lustre was an attractive material for Roman military equipment, such as brooches and the fitments of sword sheaths. The onset of the application of brass in the Eastern Alpine region was determined from the composition of brooches [29].

Brooch types change frequently according to fashion and the occurrence of particular types is well dated archaeologically. This study showed that brass appeared in some examples of the brooch type Almgren 65, while later Alesia type brooches were generally made of brass. This puts the first use of brass at around $60 \mathrm{BC}$. This dating 
is considerably earlier than the Augustan money reform of $23 \mathrm{BC}$, which was traditionally considered as the onset of brass use, largely based on the introduction of the brass coin types dupondii and sestertii. The results of the brooch analysis showed that the use of brass started about 40 years earlier.

The use of brass further implies complex relations between the Romans and their barbaric neighbours. Several swords found at the border of the Roman Empire were made in Late Iron Age (La Tène) style, though their material is brass, i.e. typically Roman. Measurements were made using an in-air proton beam in order to allow the analysis of large objects and the samples were prepared for measurement by gently polishing small areas of the material [30].

The archaeologist $\mathrm{J}$. Istenic explains the disparity between the style and use of the material as a result of involved international relations: the objects were produced in Roman workshops and intended as gifts for noblemen that lived on the border of Roman Empire and had important contact with the Romans, yet their archaic taste was still bound to the Late Iron Age forms [31].
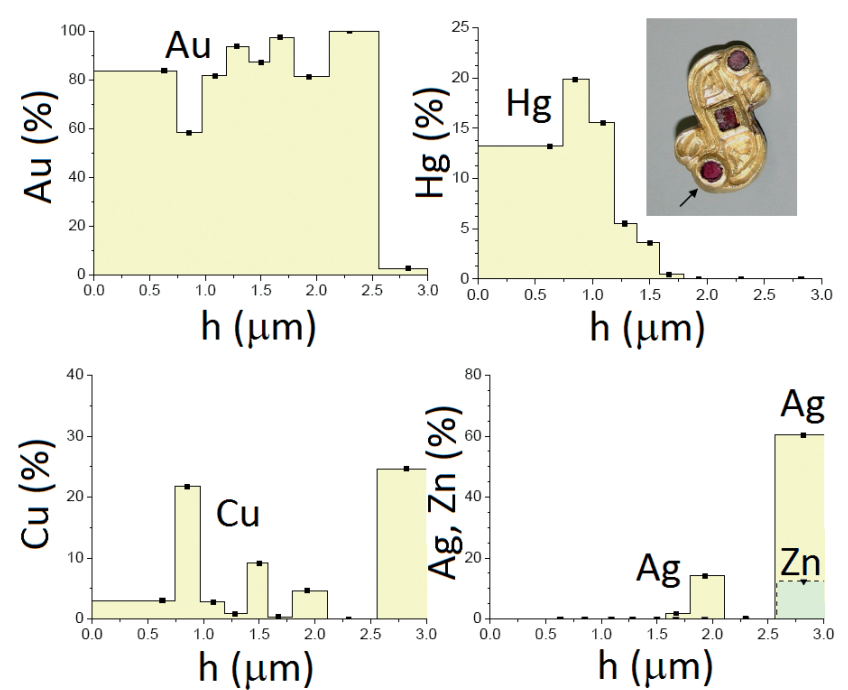

Figure 2.6: $\mathrm{Au}, \mathrm{Hg}, \mathrm{Cu}, \mathrm{Ag}$ and $\mathrm{Zn}$ concentration profiles of the gilded layer on a Late Antique brooch obtained by differential PIXE; $h$ is the distance from the surface [33]

The second example of metal analysis demonstrates identification of gilding techniques. The objects studied were from the Late Antiquity, which favoured gilded silver or bronze jewellery with inlaid garnets. The methods applied were differential PIXE and Rutherford spectroscopy with an in-air proton beam [32,33]. Figure 2.6 shows the measured $\mathrm{Au}, \mathrm{Hg}, \mathrm{Cu}$ and $\mathrm{Ag}$ concentration depth profiles of a Late Antique brooch [33].

The measurements were made at nine impact energies ranging from $2.78 \mathrm{MeV}$ to $740 \mathrm{keV}$. The gold layer is found to be about $2.5 \mu \mathrm{m}$ thick, but the gold is not pure: throughout the profile it is mixed with mercury, which undoubtedly reveals that a fire gilding or amalgamation procedure was used. For this technique, a paste of gold amalgam is applied to the object surface. The object is then heated until the mercury evaporates and a solid gold layer forms at the object surface. The evaporation of mercury is never complete; the gold layer typically contains up to $15 \%$ mercury, which then remains as a clear indication of the technique.

Gold and mercury layers may also be identified by RBS; however, the mass resolution of the experiment does not allow clear separation of the weak mercury and strong gold signals. The presence of mercury has to be confirmed using X-ray spectra. The codes for concentration profile de-convolution are still being developed, and the combined use of X-ray and backscattered particle spectra seems to be the most efficient [34].

Ion beam techniques can also be used for the identification of the surface layer of tin and silver, though the thickness of silver may exceed the range of particles at the highest impact energy, thus giving an impression that the object is made of solid silver.

\subsubsection{GLASS}

Glass is an artificial inorganic compound composed of many elements. Their relationship varies significantly over historic periods, so the analysis of glass may provide valuable historical information about the sources of raw materials and their transport routes.

Glass is generally composed of three main components: siliceous matrix, alkaline flux that lowers the melting point and alkaline earth oxides required for chemical stability. As the agents used for glass production are not chemically pure, but contain significant amounts of metal impurities, notably iron, common glass is usually coloured. The influence of iron is neutralised by decolourants, which turn valence-two iron into its three-valence form. The use of decolourants is also historically dependent.

Glass may further be coloured, yet the amounts of required colourants are so small (at the percentage level or below) that they hardly change the bulk composition.

The multi-element composition of glass is challenging for different analytical methods. The advantage of ionbeam methods is the non-destructive nature of the investigation: whole objects may be analysed at particular points, without sampling. Archaeological objects may be covered by a layer of oxides, which should be removed before measurement. Surface leaching of alkaline elements may occur and an assessment of this effect has to be made. Glass may be regarded as a mixture of metal oxides, so the lightest element to be detected is sodium.

In order to separate sodium and magnesium using the PIXE analytical method, the measurement has to be performed in vacuum or in a helium atmosphere using a thin window X-ray detector. Alternatively, low Z elements are determined by PIGE, which exploits detection of $\mathrm{Y}$-rays 
induced by inelastic proton collisions. Sodium lines are intensely produced in deeper regions of the target (below $10 \mu \mathrm{m})$, so detection of bulk sodium is straightforward. Detection of magnesium is less favourable, as the production rate of magnesium lines is lower, yet the most intense $y$-ray at $585 \mathrm{keV}$ lies very close to the $583 \mathrm{keV}$ y-ray, which exists in natural background radiation. With careful measurement, a detection limit of $0.2 \%$ magnesium can be reached, which is just sufficient for the glass of the Roman period.

Glass also contains important trace elements around strontium and zirconium, which are important indicators of raw material provenance. The sensitivity of ion-beam methods to these elements and to the rare earth elements is much lower than achievable by several chemical methods, yet useful data may be obtained from hard X-ray PIXE spectra.

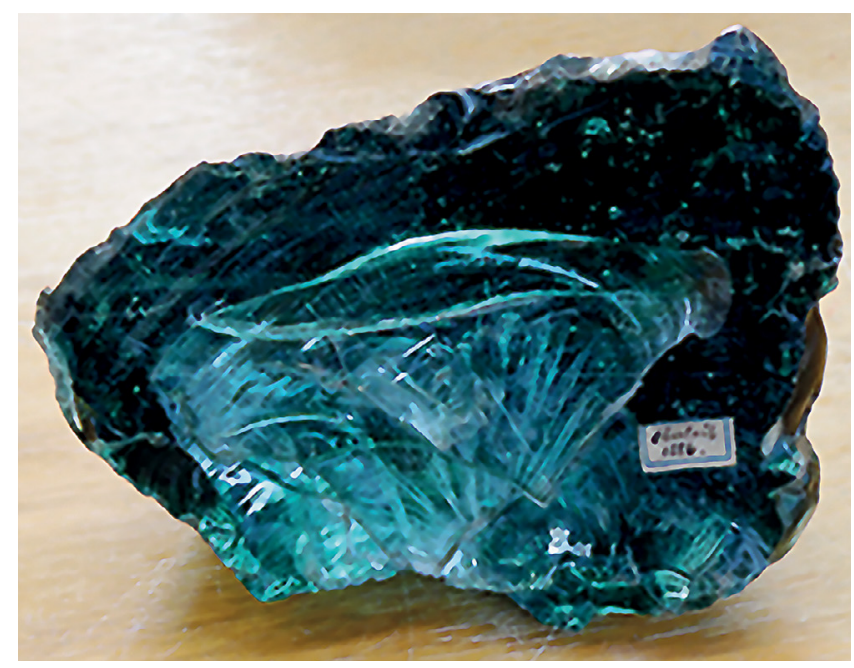

Figure 2.7: Chunk of glass discovered close to the Roman settlement Nauportus, presently Vrhnika in Slovenia [40]. Analysis based on the PIXE-PIGE method demonstrated that the material was natron-type glass characterised by sub- percent concentrations of $\mathrm{MgO}$ and $\mathrm{K}_{2} \mathrm{O}$. This indicates that the material represents primary Roman raw glass intended for further reworking in secondary workshops.

High energy $\mathrm{X}$-rays are detected by a detector with a large solid angle, simultaneously suppressing the intense low energy X-rays with an absorber. Recent glass studies [35] deduce important information from the isotopic ratios ${ }^{87} \mathrm{Sr} /{ }^{86} \mathrm{Sr}$ and ${ }^{143} \mathrm{Nd} /{ }^{144} \mathrm{Nd}$; this type of measurement is unfortunately not obtained by ion-beam methods.

Historic glasses are dominated by the choice of alkalis which are obtained from the ash of plants or from mineral deposits. The latter category involves glass produced with natron, a dried sediment from Egyptian lakes. Natronbased glassmaking developed in the area of present Egypt, Palestine and Syria and dominated glassmaking in the Greek, Roman and post-Roman world during the long period from $800 \mathrm{BC}$ to $800 \mathrm{AD}$. Glass produced in the second millennium BC in Bronze Age Egypt and
Mesopotamia was made of ash of halophytic plants of either maritime or dessert origin. The same species of plants were also used during the transition period between 800 and $1200 A D$ and represented the basis of Venetian glassmaking.

In central and northern Europe, beech ash was used instead, which produced potassium-rich forest glass. From the analytical viewpoint, it is relatively easy to distinguish between different types of glass. Sodium glasses are distinguished according to the level of magnesium and potassium impurities: natron is generally much purer than ash of halophytic plants. The concentration of sodium is small compared to potassium in forest glass. Ash was subject to different purification procedures. The most rigorous was making potash through precipitation, which can be recognised through the absence of soluble metal oxides in the finished glass objects.

The most important question regarding Roman glass is its primary production site. According to Pliny, glass was produced in the Levant area, but also in Italy, southern France and Spain. Archaeological and analytical data show that during the late Antiquity, raw glass was produced entirely in the Levant area and distributed to secondary workshops elsewhere in the Empire in the form of glass chunks. New studies based on isotope distribution suggest that raw glass was made in the western and eastern part of the Mediterranean during the Imperial period $\left(1^{\text {st }}-4^{\text {th }} \mathrm{C}\right.$. AD), but production centred in the eastern part in the late Antiquity [35].

Measurements performed at Ljubljana involved Greek glass from Apollonia Pontica [36] and Roman glass from Albania [37], Bulgaria [38] and Serbia [39]. In the latter case we identified a glass chunk (Figure 2.7) made of natron-type glass [40] and measured glass from a hilltop post-Roman settlement [41].

The results show multiple sources of raw materials for the Albanian glass which is consistent with the model of dispersed raw glass production. Glass in the later periods becomes less pure, mainly due to repeated recycling. New glass types came into use in late Antiquity, showing established commercial routes across the Mediterranean and Italy.

The first items to reveal new glass technology around $800 \mathrm{AD}$ were glass beads, produced in the Islamic East. As commercial items they spread much faster than the raw glass. A systematic study of glass beads excavated in Slavic graves in Slovenia [42] showed two significant groups: beads made of natron-type glass according to the Roman tradition and beads made of the ash of halophytic plants (Figure 2.8). The occurrence of the latter is important for dating: certain graves in Slovenia were dated to the $7^{\text {th }}$ and $8^{\text {th }} \mathrm{C}$. AD in early studies, but now have to be dated later, to the first half of the $9^{\text {th }} \mathrm{C}$. AD because of the presence of glass beads made from plant ash. This dating is consistent with the dating of the Köttlach culture in central Europe. 


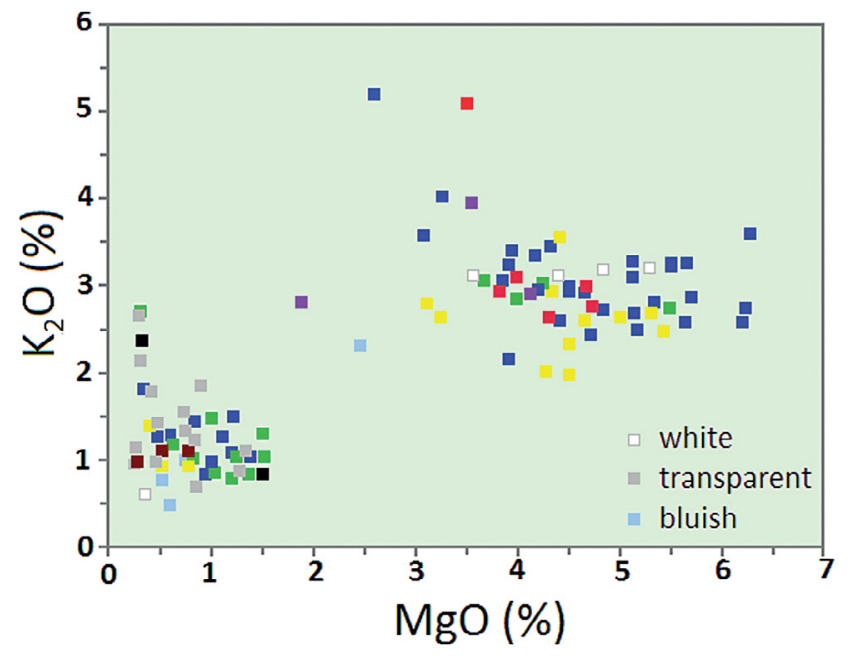

Figure 2.8: Concentrations of potassium and magnesium oxides in early medieval glass beads excavated from graves in Slovenia, determined by a combined PIXE-PIGE method [42]. Glass made from Egyptian natron (lower left, with low magnesium and potassium values) and glass made from ash of halophytic plants (upper right, high magnesium and potassium) form two distinct groups. The latter type of glass spread after 800 AD. This information can be used to date the graves.

Production of glass made from the ash of halophytic plants, collected at various Mediterranean coastal locations, peaked in the period starting roughly in the $13^{\text {th }}$ c. and ending in the $17^{\text {th }} \mathrm{c}$. The main producer was Venice.

Glass from Venice is known as Venetian glass. Glass produced in the same manner, but outside Venice, is labelled as glass à façon de Venise. For historical sites that show abundant glass finds it is important to know if they produced glass locally or if they imported it from Venice. The same question arises regarding Ljubljana, where about 800 glass fragments are kept in the National Museum and local production of glass is documented in historical records. The analysis using the PIXE/PIGE method of more than 300 specimens showed two distinct groups [43].

The comparison with other glasses from Western Europe and Italy showed that the two groups are universal and imply two different ash sources $[44,45]$. One is undoubtedly Venetian, but interpretations for the other group differ: though it also contains samples of Venetian origin, it may also indicate glass à façon de Venise [45]. However, as the same glass type was also identified among the much earlier medieval glass beads and glass around the Aral Sea [46], this type of glass may only suggest a specific ash source from a certain, presently unidentified plant. Further development of glass technology in Venice resulted in a much finer and transparent cristallo glass. In the $17^{\text {th }} \mathrm{c}$. cristallo was also produced outside Venice, for example in Antwerp.

The switch to the new technology was nicely observed among the glass of the Albanian city of Lezha: besides the two groups of common Venetian glass, a distinct group was identified that was made of purer silica and alkalis purified by precipitation. The glass was further discoloured by arsenic, which replaced manganese in the former Venetian glass [47].

Individual properties of particular glass producers may also be identified for the glass of the late $19^{\text {th }}$ and early $20^{\text {th }}$ c. A study on uranium-pigmented and red glass kept at the National Museum of Slovenia showed that the glasses were produced in three different places: one was very likely located in the present Czech Republic, while the other two were in Slovenia, one being identical with a present day glass factory in Hrastnik [48].

\subsubsection{CERAMICS}

Archaeological ceramics have a coarse-grained structure which renders analysis by millimetre beams unreliable, unless the samples are finely ground and homogenised. The advantage of non-destructive ionbeam methods is thus lost, and the powder obtained can be equally well analysed by wet chemical methods or in the form of pellets that can be analysed by a variety of methods. Using a particle microbeam on the cut and polished surface it is possible to identify particular mineral species. On the other hand, bulk analysis can be successful on objects made of cream-coloured ceramics [49]. The material is sufficiently homogeneous that measurements in selected, glaze-free, areas can give representative results.

Cream-coloured ceramics were invented in England in the $18^{\text {th }} \mathrm{C}$. as an inexpensive substitute for porcelain. Northern Italy (then part of the Austrian empire) soon

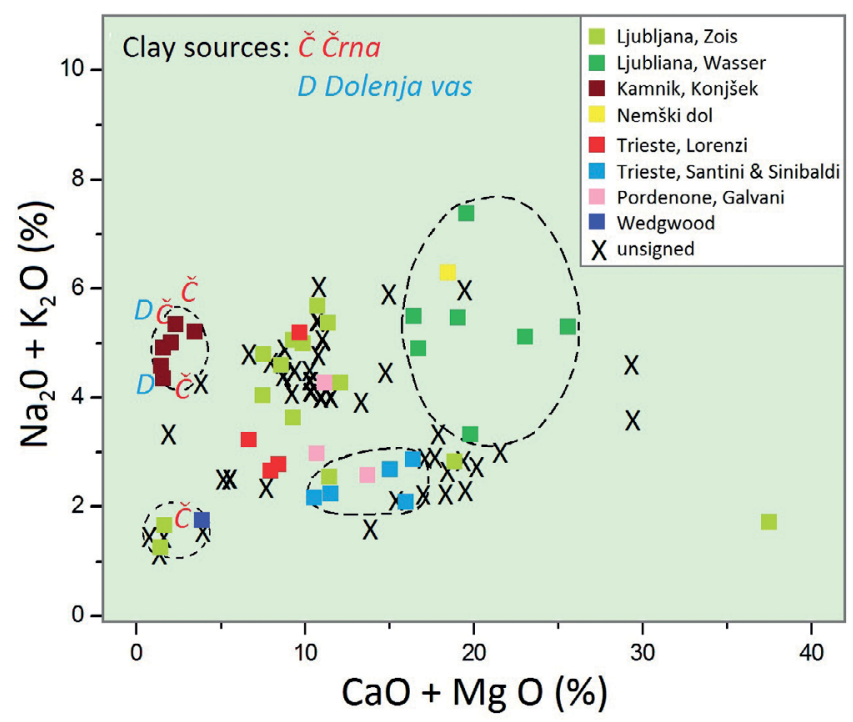

Figure 2.9: Compositional groups of cream coloured ceramics produced during the $19^{\text {th }} \mathrm{C}$. in the present day territories of Northern Italy and Slovenia, determined by PIXE-PIGE analysis. Items corresponding to two separate sources of white clay extracted in Slovenia both have low $\mathrm{CaO}+\mathrm{MgO}$ concentrations. 
followed as a significant production centre, as did the neighbouring countries of present day Slovenia where two deposits of white clay were discovered. The composition of selected objects from the National Museum of Slovenia was measured by the combined PIXE-PIGE method following the procedures developed for glass analysis.

A statistical analysis based on principal components was able to distinguish particular producers, but was not able to distinguish between the two clay sources (Č. Črna and $D$. Dolenja vas in Figure 2.9). The differences between the producers arose from mixing the primary batch with different minerals, notably limestone. It was possible to observe that one producer (S. Zois - lime green) largely experimented with different mixtures, as documented in his notebooks.

\subsubsection{PRECIOUS STONES}

Precious and semi-precious stones were popularly worn in Antiquity: emeralds by Romans and garnets by their barbaric successors. Analysis of precious stones is similar to the analysis of glass as their composition can be interpreted as a combination of metal oxides. PIGE is used for light elements and PIXE for medium and heavy elements. Emeralds contain a known fraction of beryllium which can be measured either directly or taken into account numerically for the calculation of matrix effects.

The provenance sites of precious stones are of particular interest as they indicate the extension of commercial routes first established by the Romans. However, the determination of emerald sources is not unambiguous [50] and additional mineralogical and gemmological investigations have to be carried out.

For example, the fluid channels in a set of emeralds excavated in a Roman grave from Slovenia point to a source in Egypt, while emeralds from another grave may be traced to Afghanistan [51]. Interestingly, the emeralds were not from a nearby source at Habachtal in Austria.

Elemental analysis proved useful for provenance studies of garnets. The majority of garnets in Europe came from India and Sri Lanka. There was a disruption of transport routes by the end of the $6^{\text {th }} \mathrm{C}$. AD due to the Sassanid seizure of the Arabian peninsula. Garnets from Bohemia (present day Czech Republic) and Portugal were subsequently used in the Merovingian kingdom [52-55]. Interestingly, Bohemian garnets are absent in the territory of present day Slovenia despite the proximity of the sites.

The reason is very likely to lie in the incursions of Avars and Slavs who did not follow the fashion of their Germanic predecessors [55]. But also in the West, the low quality of new stones heralded a decline in garnet embroidered jewellery.

\subsubsection{PIGMENTS AND PAINTINGS}

In-air particle beams are an ideal tool for the investigation of paintings and the identification of metal-based pigments. Low intensity beams and short exposure times cause no radiation damage to pigment layers. A brief measurement can identify the type of pigment, which may have a historic context, and can discover later repairs and curator treatments. For example, a very short measurement can distinguish between lead, zinc or titanium white; the third is a pigment introduced in the $20^{\text {th }} \mathrm{C}$. The measurements are particularly interesting for $19^{\text {th }} \mathrm{c}$. paintings, since the rapidly developing chemical industry of the period introduced many new synthetic pigments.

Though pigment identification can be equally well established by a much cheaper X-ray apparatus, the main advantage of ion beam methods is a combined application of different methods, including differential measurements. Performing a series of measurements in the same spot, it is possible to determine the composition of particular paint layers. For example, using differential PIXE it is possible to determine the composition of the layer with the signature of the painter [56].

\subsubsection{PAPER}

Records on paper preserve a crucial part of human cultural heritage. Historical archives contain many hand-written documents with iron-gall ink on an acid paper. With time, the ink acts corrosively on the paper and virtually destroys it. Ion beam methods have been successfully applied to detect iron-based inks and can also provide the concentrations of other heavy elements such as copper and zinc. Though the methods cannot be used for the precise identification of the chemical state of these elements, they are efficient in the fast detection of iron-gall inks [57]. They can also distinguish between particular inks and thus between particular writers.

PIXE on graphic works can provide other important information: e.g. this technique can identify the types of pencils used (graphite, lead or silver) or pigments, such as lapis lazuli, in hand-painted incunabula. It is also possible to identify printing inks and a number of printing offices.

\subsubsection{CHINESE MING POTTERY (FROM ANGKOR THOM)}

The purpose of the investigation was to assess the possible origin of Chinese pottery shards (presumably dating to the Ming dynasty) found in excavated material from an ancient pool at the royal palace grounds of Angkor Thom. As the former imperial city was abandoned shortly after its sacking by the Thai armed expedition in $1431 \mathrm{AD}$ it seems most probable that the artefacts found on the grounds had been imported by the Royal court while still at Angkor Thom, that is some time before that date. 
The study aimed to separately analyse the composition of the glaze and painted sections containing cobalt.

A further aim was to find the possible origin of the kilns in China where the shards found were manufactured. This would be done by comparing the composition of the cobalt with reported measurements of elemental composition made with PIXE on shards found at various kiln locations in China.

The shards were sliced into thin sections and examined with a microscope coupled to a camera to allow identification of the blue pigment depositions and the glaze. The samples were placed in aluminium holders and irradiated with a proton beam of about $150 \mathrm{pA}$, focused to a $1 \mu \mathrm{m}$ spot using an Oxford triplet of magnetic quadrupole magnets. The proton beam energy of the Tandetron accelerator at the Řež Microprobe Facility, was varied between 2 and $3 \mathrm{MeV}$ according to the molecular weight of the elements studied in a particular experimental session.

The X-ray emissions from irradiated shard slices were recorded using an $80 \mathrm{~mm}^{2} \mathrm{Si}(\mathrm{Li})$ detector. Furthermore, the RBS spectra were measured simultaneously using a PIPS detector. The diameter of the focused beam was equivalent to 9 pixels of the resulting 2D-maps, which showed the concentration of each particular element. Up to 20 elements were determined during each measurement.

The maps of individual elements were constructed from their emission spectra. The advantage of the measurements reported here over those reported previously was their superior spatial resolution which enabled pertinent sites to be targeted, reducing the partial volume effects associated with measurements using wider beams.

The 2D maps of elemental composition revealed a high calcium content, greater than $10 \%$, in the pottery glaze. This rather high concentration is compatible with values for calcium compositions reported for Chinese pottery of the Ming dynasty, presumably produced prior to 1431 AD.

The cobalt pigment sections showed high concentrations of arsenic. Arsenic is a major constituent of asbolite that was presumably the source of the pigment imported during the Ming dynasty from Persia. Cluster analysis of the elemental compositions determined for the glazes on numerous shards showed the feasibility of ascertaining their provenance. From the elemental composition of the cobalt pigment and glazes, it appears that the pigment was most likely imported from Persia and that the shards analysed were manufactured in kilns at two distinct locations in China [58].

\subsubsection{TYCHO BRAHE - WAS HE MURDERED OR WAS HE NOT?}

World-renowned Renaissance astronomer Tycho Brahe died on 24 October 1601, after 11 days of sudden illness. Several conspiracy theories, suggesting mercury poisoning, were aired shortly after his death. In 2010, Brahe's grave in Prague was reopened and samples of his bones, hair, teeth and textiles were procured and analysed. The hairs with identifiable roots (Figure 2.10.) were cut into sections about $5 \mathrm{~mm}$ long and washed using the IAEA recommended procedure [59].

Sectioned samples from 20 to 25 individual hairs weighing 200 to $300 \mathrm{mg}$ were sealed in pre-cleaned high-purity quartz ampoules and irradiated for 20 hours at the Czech Academy of Sciences LVR-15 nuclear reactor in Řež at a thermal neutron fluence rate of $3 \times 10^{13} \mathrm{~cm}^{-2} \mathrm{~s}^{-1}$. The ${ }^{203} \mathrm{Hg}$ produced was separated after 2 to 3 weeks decay using an RNAA procedure (see chapter 3) [59] based on $\mathrm{Hg}$ extraction with $0.01 \mathrm{~mol} \mathrm{~L}^{-1}$ $\mathrm{Ni}$ diethyl dithiocarbamate $\left(\mathrm{Ni}(\mathrm{DDC})_{2}\right)$ and measured by high-resolution $\gamma$-spectrometry. Unsectioned hair samples were also analysed by $\mu$-PIXE, using a Tandetron 4130 $\mathrm{MC}$ accelerator with a $2.6 \mathrm{MeV}$ proton beam focused to a diameter of $1.5 \mathrm{~mm}$. Multiple scans were performed over $500 \mathrm{~mm}$ sections of hair at a $0.1 \mathrm{nA}$ beam current for 1 to 3 hours.

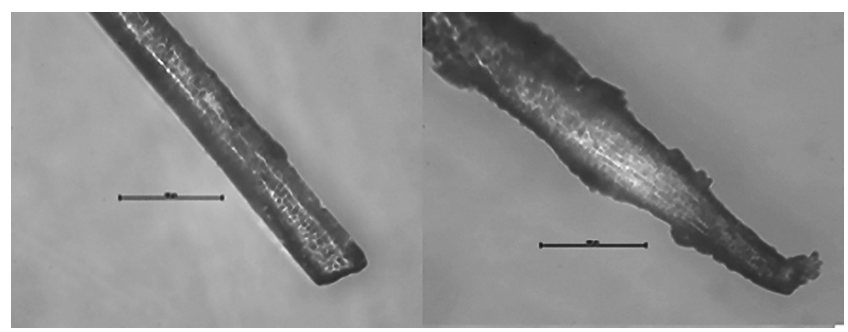

Figure 2.10: The hair tip and root from a sample of Tycho Brahe's hair. The inserted scale is $100 \mu \mathrm{m}$.

Figure 2.11 shows excellent agreement between the RNAA and $\mu$-PIXE results for one analysed hair sample and compares the values found with the median and range of $\mathrm{Hg}$ content in the contemporary unexposed population. Hair provides a lasting record of exposure to trace metals over the last few months of life. The hair samples analysed in this study reflect the $\mathrm{Hg}$ intake over approximately the last 2 months prior to the death of Tycho Brahe, assuming the most frequently cited hair growth rate of $10 \mathrm{~mm}$ per month [60].

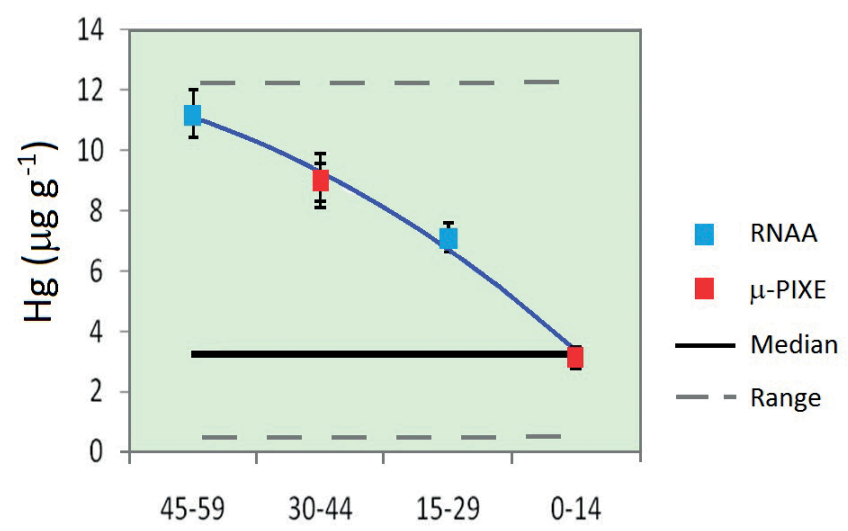

\section{Days before death}

Figure 2.11: Time dependence of the $\mathrm{Hg}$ content in one sample of Tycho Brahe's hair measured using RNAA and $\mu$-PIXE techniques, compared to the median (black solid line) and range (grey dotted lines) of $\mathrm{Hg}$ content in contemporary unexposed populations. 
The highest $\mathrm{Hg}$ values found are slightly above the median of normal values, and well within the normal range. Their reduction towards Brahe's death, suggests that he was not exposed to excessive $\mathrm{Hg}$ doses shortly before his death (no acute poisoning).

Analysis of Brahe's bones also revealed no long-term exposure to $\mathrm{Hg}$ (no chronic poisoning). Thus the analyses carried out prove that the famous astronomer was not poisoned by $\mathrm{Hg}$ and a murder scenario has become a much more remote possibility than previously thought.

\subsubsection{ORIGINS OF THE SARMIZEGETUSA GOLD BRACELETS}

It is already well-known that trace elements are more significant for determining the provenance of archaeological artefacts than the main components for gold, silver, obsidian or copper-bronze items. Because a high sensitivity elemental analysis of valuable museum objects is quite difficult - especially expensive due to transport and security problems - an adequate solution is to complete in-situ XRF analysis in museums. Micro samples are taken and studied using advanced micro-spectrometric X-ray methods. The micro-PIXE technique, which is sensitive at a level of a few ppm and has excellent lateral resolution, is capable of microinclusion detection and is one of the best available methods of providing information on the provenance of archaeological artefacts.

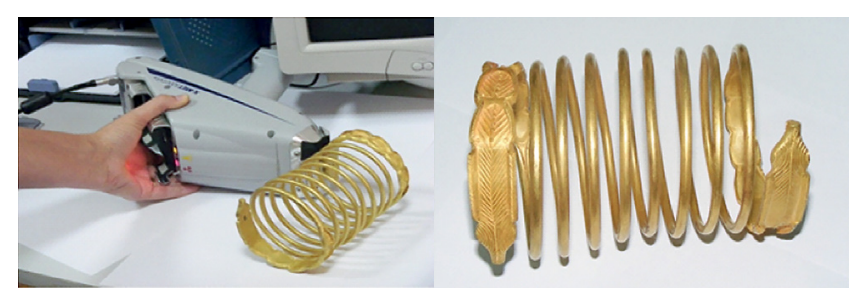

Figure 2.12: Dacian spiral gold armbands studied by XRF analytical methods.

For the authentication of ancient gold artefacts (jewellery and coins) found on Romanian territory, the most likely use of unrefined Transylvanian gold must be considered.

In ancient times, and up to the Middle Ages, the most important source of gold was placer deposits. Alluvial gold is derived from weathered rocks containing veins of gold deposits. Gold is highly resistant to weathering; its particles are washed down mountains along with weathered rocks, and are subsequently deposited in the sand and gravel of rivers.

Naturally occurring gold contains several impurities, most notably silver and, in a much smaller proportion, copper. During the weathering and transport of gold particles, silver and copper are more susceptible to dissolution or leaching, depending on the $\mathrm{pH}$ value of the environment. Consequently the overall silver and copper content of alluvial gold is somewhat less than the content of the vein gold from which it is derived.

A relevant demonstration of the use of alluvial gold in prehistoric times in Transylvania - one of the richest gold producing regions in Antiquity - is a series of complex studies performed between 2007 and 2012 on Dacian gold artefacts, including 13 spiral gold armbands, see figure 2.12. (These artefacts were discovered illegally in the Sarmizegetusa area by treasure hunters using metal detectors.) The compositional analysis of the $\mathrm{Au}-\mathrm{Ag}-\mathrm{Cu}$ content of the armbands confirmed they were made of native Transylvanian gold. The presence of tin indicated an alluvial deposit. Some antimony, which is one of the main fingerprints of a primary Transylvanian gold vein, was also observed [61-64].

\subsubsection{PROVENANCE OF LAPIS LAZULI}

Lapis lazuli is a semi-precious blue stone which has been used widely since antiquity for many different purposes. However, information regarding the quarries used by different civilisations to extract the mineral and information about its trade in ancient times remains scarce.

Historical sources of lapis lazuli are located in inaccessible places, such as the Afghan and Pamir Mountains and stones were transported for thousands of kilometres. Unfortunately, these trade routes are largely incomplete and unknown. Only a few sources exist in the world because of the restricted compositional and physical constraints in which lapis lazuli can form [65]. Therefore assigning sources of raw material to man-made objects can help historians and archaeologists reconstruct ancient trade routes.

A systematic study (see Figure 2.13) of this fascinating stone compared the physico-chemical properties of rocks from four different sources (Afghanistan, Tajikistan, the Lake Baikal region and Chile) [66-72]. Many analysed lapis lazuli rocks and objects come from the collections of the Museo di Storia Naturale (University of Florence, Italy). About fifty pieces of lapis lazuli are conserved in this collection. Half of these are blocks of rock, which are rough or partially polished, and the other half consist of carved objects of exquisite workmanship and fragments or whole tesserae for inlays.

Recently other groups have started to study lapis lazuli, both as rock and as ultramarine blue pigment, for provenance identification, using different approaches and obtaining interesting results [73-80].

Due to the paragenetic mineralogical heterogeneity of lapis lazuli, the single mineral phases were analysed to search for markers useful for a provenance study. Since 


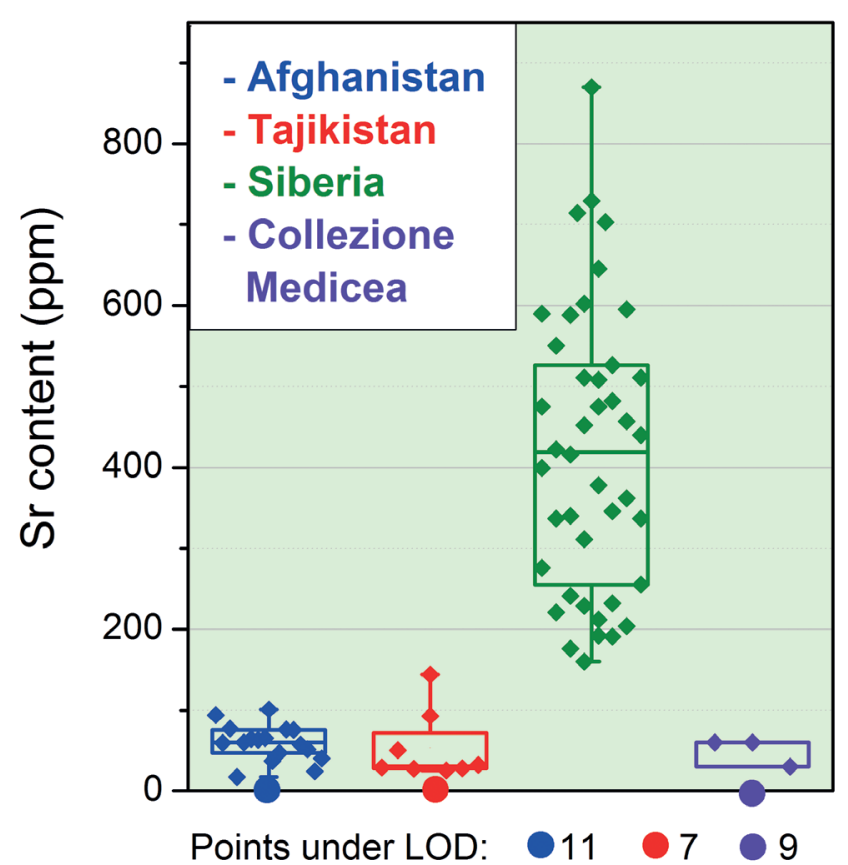

Figure 2.13: Sr content in diopside minerals from various sources. The boxes contain the central $50 \%$ of measurements, while the vertical bars indicate the full range of the measured data. The horizontal lines in the boxes divide the data in two identically sized groups. The large dots at zero content indicate points with $\mathrm{Sr}$ content below the detection limit.

crystal dimensions vary from a few microns to hundreds of microns, high spatial resolution techniques are mandatory. IBA techniques with an external proton microprobe were used. External IBA allows for non-invasive, multi-technique (PIXE, PIGE, and ion beam induced luminescence IBIL) studies of objects of almost any shape and dimension; see for example Figure 2.14.

The study focused on identifying markers, such as the presence (or absence) of a specific mineral phase in the stone, the concentration of trace elements in a mineral or the luminescence features of a particular crystal, in order to identify the provenance of the stone.

A multi-technique approach, including optical microscopy, SEM-EDX, cathodo-luminescence, Raman spectroscopy and in-vacuum IBA is used to identify markers in the analysis of rocks from a particular provenance. A non-invasive IBA analysis is performed on the artworks containing lapis lazuli and the previously discovered markers are used to identify the origin of the stone used.

Some of the markers identified on stones have been successfully used in the study of six precious artworks from the Collezione Medicea (see Figure 2.14) made in lapis lazuli [73].
The identification of the regions to be analysed was carried out using a broad beam ion luminescence microscopy setup [81]. This gave a preliminary indication of the distribution of different mineralogical phases and allowed the biggest and most homogeneous crystals to be selected.

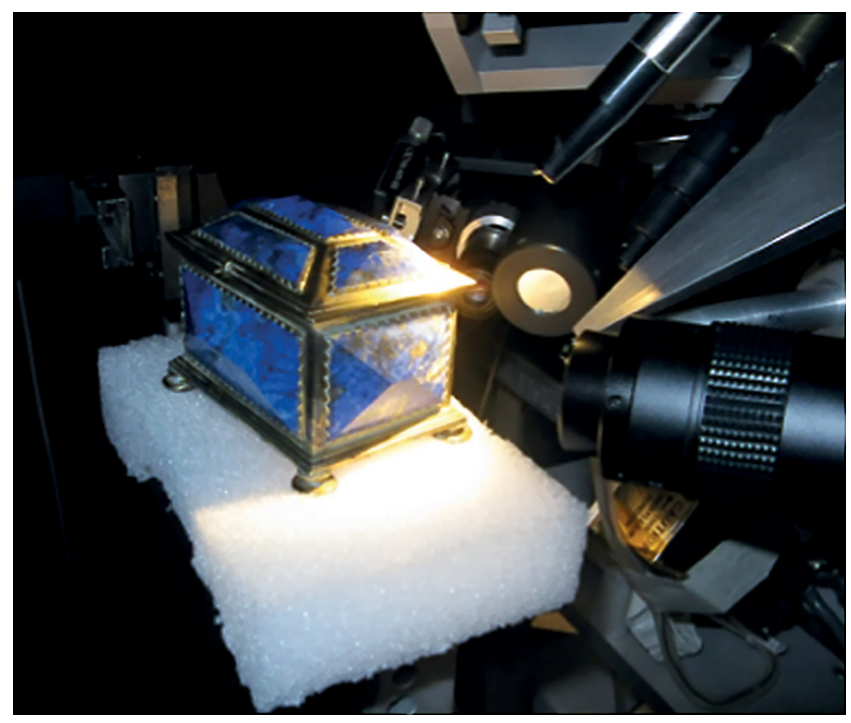

Figure 2.14: An artwork from the Collezione Medicea photographed during an external microbeam IBA analysis carried out at INFN-LABEC, Florence.

After this preliminary selection, ion microbeam analyses ( $\mu$-IBIL and $\mu$-PIXE) were performed on selected crystals. Ion luminescence allowed a Chilean origin to be excluded because of the absence of wollastonite, a mineralogical phase typical of Chilean provenance. Wollastonite is characterised by a particular luminescence pattern which was not observed in the IBIL measurements on the artworks.

The rocks from the three Asian provenances are all characterised by the presence of diopside, a luminescent mineralogical phase commonly present in lapis lazuli. The content of trace elements inside this phase in all the artworks studied was measured using $\mu$-PIXE and compared to the rock database.

Among the detected elements, Sr has the sharpest capability to discriminate between different provenances: a quantity higher than $150 \mathrm{ppm}$ has only been detected in Siberian samples. In the Collezione Medicea artworks, the $\mathrm{Sr}$ content is always below 100 ppm, so the Siberian provenance can be excluded (Figure 2.13.).

The amount of other trace elements (titanium, vanadium, chromium) in this phase, combined with some luminescence features, allowed an Afghan origin to be ascribed to the material used for five of the six artworks studied. For the sixth artwork a Tajikistan provenance cannot be excluded and a further analysis, e.g. checking markers related to other phases such as pyrite, is needed to confirm or reject an attribution of origin to the Afghan provenance. 


\subsubsection{LA MADONNA DEI FUSI, BY LEONARDO DA VINCI}

The depth sequence of elements in paintings can be obtained using differential PIXE (d-PIXE). PIXE measurements are made at the same place with a range of beam energies. The sequence of layers can be inferred, at a semi-quantitative level, by comparing the X-ray spectra collected at different incident beam energies.

The interpretation of d-PIXE data is complicated by several factors. For example, the number of layers and their thickness is unknown, so that the choice of the beam energies to separate the contributions from the various layers is not straightforward. Moreover the particle energy distribution becomes larger with depth, so that a clear-cut discrimination of the layers cannot be readily achieved.

A PIXE/d-PIXE/PIGE study was performed on the famous painting by Leonardo da Vinci the Madonna dei fusi (ex-Redford version) [82,83], shown in Figures 2.15 and 2.16. This study aimed to discover the peculiar features of his painting technique. Particular care was devoted to the characterisation of the protective varnish and the blue pigments, in both the original and the restored parts.

Very safe experimental conditions were adopted, in order to avoid any risk of damage: the $2.7 \mathrm{MeV}$ proton beam was extracted into a He atmosphere, with a beam current of some tens of $\mathrm{pA}$ in measurements which lasted a few hundred seconds. The beam was about $1 \mathrm{~mm}$ in diameter.

The varnish covering the Madonna dei fusi made it difficult to detect light elements which are characterised by low energy $\mathrm{X}$-rays. The lower the energy, the higher the X-ray absorption. In addition, the varnish contained many trace elements, detected by PIXE measurements, which constituted a source of "contamination" for the pigment identification.

The varnish thickness was estimated by calculating the range of protons in organic material at the highest beam energy where the contributions from the pigment layer were not yet visible. Values ranged from 30 to $50 \mu \mathrm{m}$.

The first step of the study was the determination of the varnish composition and thickness. As the beam energy is reduced to the point where the contribution to the spectrum from elements characteristic of the paint layers disappears, it can be assumed that the protons stop in the overlying varnish.

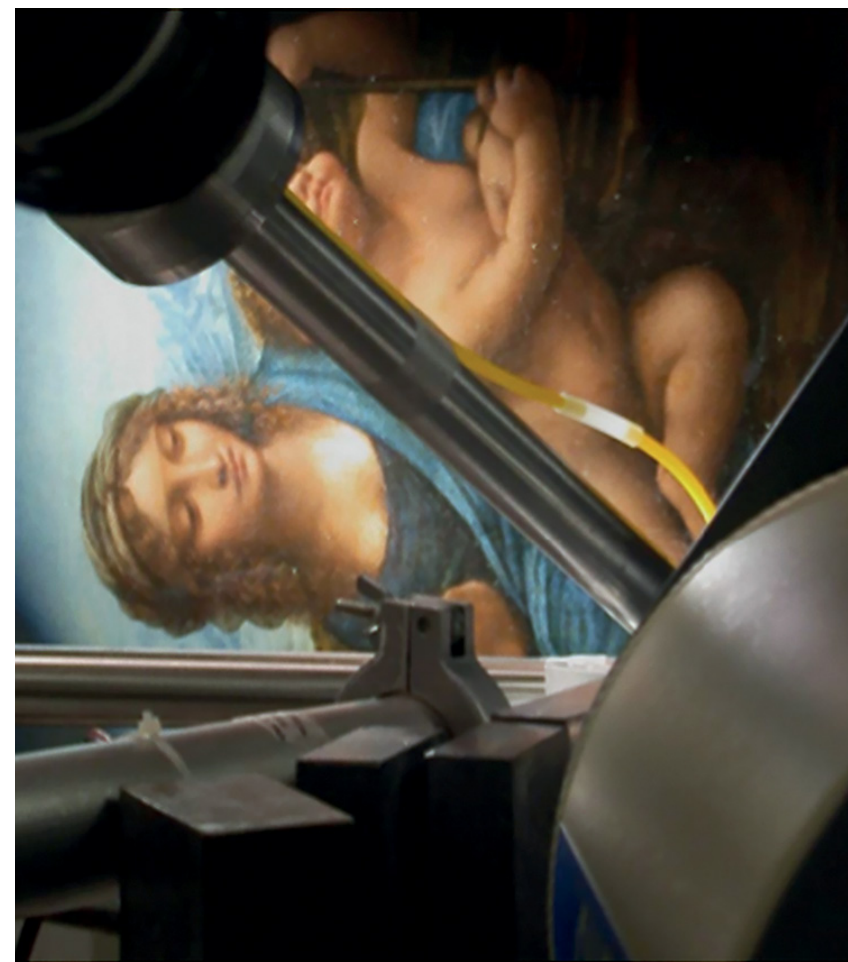

Figure 2.15: The Madonna dei fusi at the PIXE-PIGE set-up in Florence.

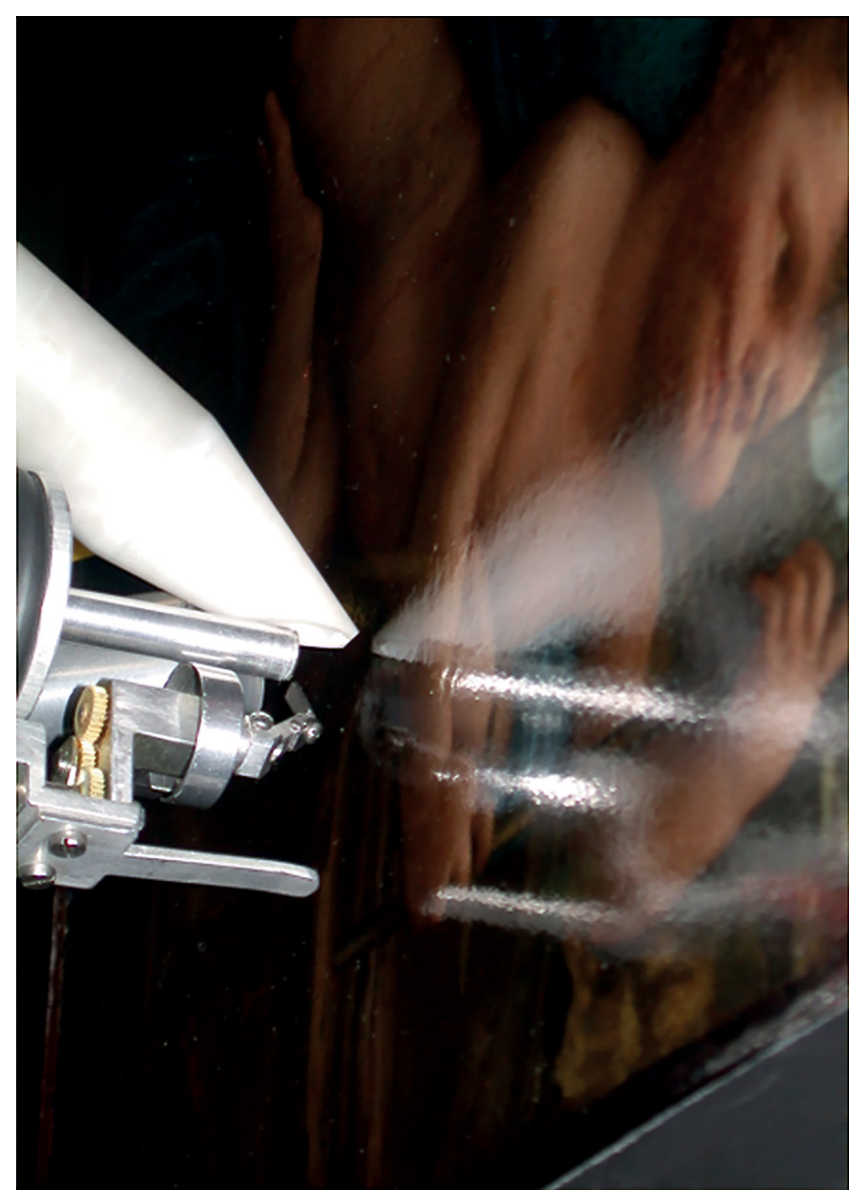

Figure 2.16: The Madonna dei fusi during the measurements. The protective varnish is evidenced by the mirror effect. 
In the second phase, we carried out a PIXE analysis of some of the blue areas. Large quantities of $\mathrm{Zn}$ and Co (the latter associated with relevant amounts of $\mathrm{Al}$ ) were detected and were attributed to the presence of zinc white and cobalt blue (cobalt aluminate). These pigments have been used only since the beginning of the $19^{\text {th }} \mathrm{C}$. and consequently those areas were identified as restorations.

When bombarding other blue areas, only a large amount of $\mathrm{Pb}$ was found, clearly associated with lead white; no elements characterising a blue pigment were detected by PIXE. The use of lapis lazuli for these blue zones could only be hypothesised: the use of the most probable periodcompatible alternative blue pigment (azurite, copper carbonate) was excluded, since $\mathrm{Cu} X$-rays would have been detected even in the presence of the varnish. Due to their rather high energy (about $8 \mathrm{keV}$ ), $\mathrm{Cu}$ X-rays have a good transmission through some tens of microns of organic material.

A direct demonstration of the use of lapis lazuli is typically achieved through the detection of $\mathrm{Na}$ X-rays. $\mathrm{Na}$ is the fingerprint of lazurite, the mineral responsible for the beautiful blue colour of the stone. Unfortunately, the presence of the varnish over the pigments makes it difficult, or even impossible, to detect light elements: the lighter the element, the lower the energy of the produced $X$-rays and the higher their absorption in the varnish. This is especially true for $\mathrm{Na}$, the lightest element detectable by external PIXE. For instance, $10 \mu \mathrm{m}$ of organic varnish (a value which can be considered a lower limit for the thickness of a varnish) absorbs more than $97 \%$ of the $1.041 \mathrm{keV}$ Na X-rays.

The highest-Z element of lazurite, $S$, which is less affected by absorption, is hard to identify in the presence of a large amount of $\mathrm{Pb}$ (as in this case, where the blue pigment was mixed with lead white), as the $\mathrm{Pb}_{\mathrm{Ma}}$ lines $\left(\mathrm{E}_{\mathrm{Ma}}=2.345 \mathrm{keV}\right)$ largely overlap the $\mathrm{S}_{\mathrm{Ka}}\left(\mathrm{E}_{\mathrm{Ka}}=2.308 \mathrm{keV}\right)$ lines.

Finally, detecting Al and Si by PIXE cannot be considered a fingerprint of the presence of lapis lazuli, since these elements are found in many other pigments which may be added to the paint layer e.g. to modify the chromatic shade.

The PIGE method was successfully applied to the study of the blue pigment of la Madonna dei fusi. All the spectra acquired in the original areas showed a strong peak at $441 \mathrm{keV}$, while those corresponding to the restored zones showed no peak at all in the same energy interval. This directly demonstrated Leonardo's use of the lapis lazuli pigment, notwithstanding the overlying protective varnish. To the best of our knowledge, this is the first time this method has been used to obtain evidence of the use of lapis lazuli blue where a protective varnish is present.

The PIGE technique provided a direct demonstration of Leonardo's use of lapis lazuli blue in the la Madonna dei fusi.

\subsubsection{RITRATTO DI GENTILUOMO, BY ANTONELLO DA MESSINA}

A complete characterisation of the materials and the structure of paintings is hard to achieve, due to their heterogeneous nature. The composition and structure of the Ritratto Trivulzio by Antonello da Messina was characterised in a non-invasive and non-destructive way by combining PIXE, differential-PIXE (d-PIXE) and scanning-mode PIXE (s-PIXE).

At the LABEC external scanning microbeam in Florence, beam spot sizes of down to about 7 microns can be obtained on samples in a He atmosphere, by extracting the ion beam through a $100 \mathrm{~nm}$ thick $1 \times 1 \mathrm{~mm}^{2} \mathrm{Si}_{3} \mathrm{~N}_{4}$ window. A target monitoring system uses three TV cameras with adjustable magnifications for sample positioning and real time monitoring. The beam charge monitor detects Si X-rays produced by the beam in the exit window. An Ion Luminescence (IL) apparatus for the simultaneous acquisition of IL/ PIXE/ PIGE/ RBS spectra and maps is also used. IL is a natural complement of the more common IBA. This configuration is well suited to various applications in the field of Cultural Heritage.

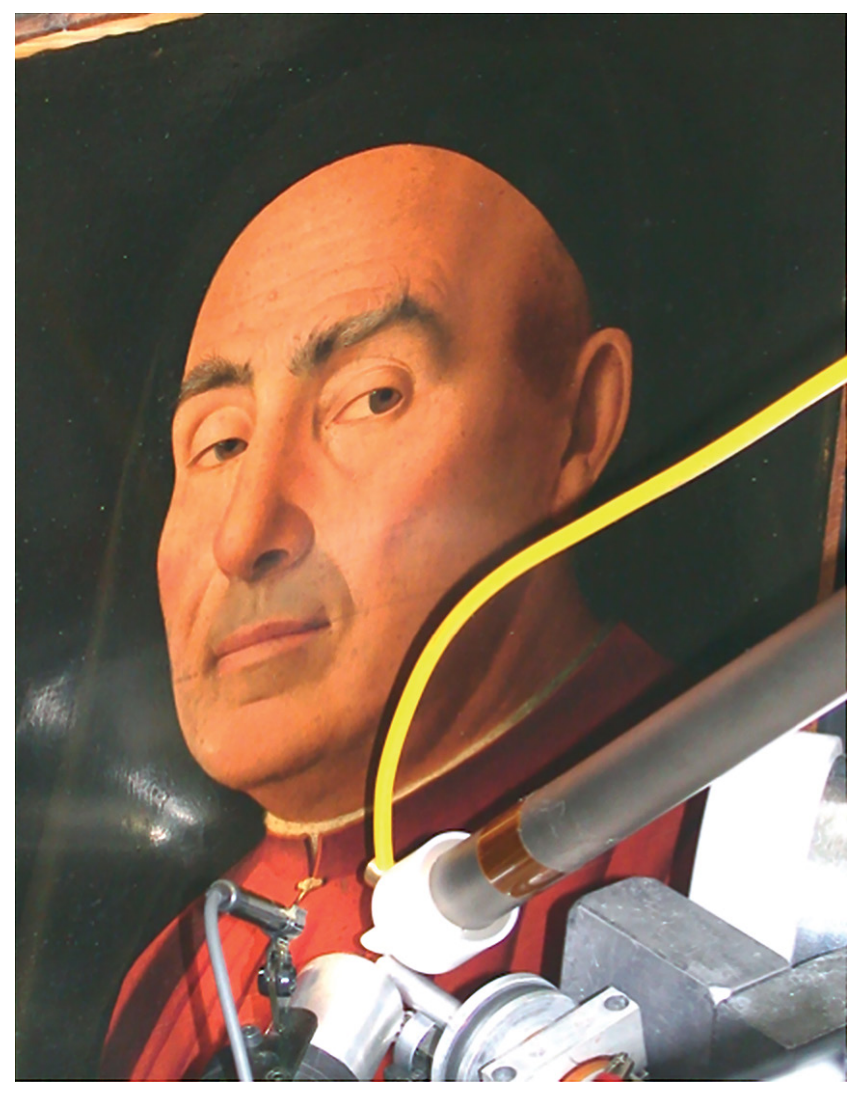

Figure 2.17: The painting Ritratto Trivulzio by Antonello da Messina $\left(15^{\text {th }} \mathrm{c}\right.$.) during measurements at the LABEC external microbeam in Florence. 
Extensive PIXE investigations were performed at the LABEC laboratory on the painting Ritratto Trivulzio by Antonello da Messina [84], one of the great Italian masters of the $15^{\text {th }} \mathrm{c}$. and a pioneer in modern oil painting; see figure 2.17 .

Non-destructive and non-invasive external beam measurements in a He atmosphere were carried out. Single-spot mode PIXE was first used for a characterisation of different areas of the painting. Then differential PIXE was used to obtain information about the layering. Finally elemental imaging, by scanning PIXE, was carried out to solve some specific problems which arose during the previous PIXE and d-PIXE studies. Here are some of the more interesting results.

The combination of PIXE, d-PIXE and s-PIXE provided information to restorers and art historians which was useful for the identification of the materials used by Antonello and to unveil some aspects of his painting technique.

- The gentleman's dark head-cloth was made of a Cubased pigment, most likely azurite (PIXE).

- In the less dark areas of the head-cloth $\mathrm{Pb}$ is associated with $\mathrm{Cu}$, suggesting the use of lead white (PIXE).

- The dark background was mainly composed of a Cubased pigment (azurite) (PIXE).

- The thickness of the Cu-based layer was found to be of the order of 30-45 $\mu \mathrm{m}$ (d-PIXE).

- The presence of $\mathrm{Sr}$ in a deep layer was explained by hypothesising the use of calcium sulphate, a typical preparation for wood paintings (d-PIXE).

- The use of cinnabar ( $\mathrm{HgS}$ ) as the main red pigment for the mantle was apparent from all the spots analysed in this area; all spectra were indeed dominated by $\mathrm{Hg}$ X-ray peaks (PIXE).

- The whitish button of the gentleman's collar was made using $\mathrm{Pb}$-based pigments painted over a red layer made with cinnabar (d-PIXE).

The whole area of the mantle was very inhomogeneous (s-PIXE).

In particular, the surface was unexpectedly characterised by darker spots of sub-millimetre size.

Thanks to the use of the high spatial resolution and the imaging capabilities of s-PIXE, it was possible to ascertain that the $\mathrm{Al}$ and $\mathrm{K}$ originated from a thin layer over the cinnabar. For the restorers, this was a strong indication of the use of a red lake pigment (prepared by reaction of the organic dyestuff with potash alum) over the cinnabar. Again according to experts, the spotted effect was probably due to shrinking of the lake during drying (s-PIXE).

\subsubsection{DATING GALILEO'S WRITINGS}

Until recently ( $19^{\text {th }} \mathrm{c}$.), the standard ink in Europe was iron gall ink. This ink was generally prepared by adding iron sulfate to a solution of tannic acid; see Figure 2.18. The gallotannic acid was usually extracted from oak galls, hence the name. Inks used in ancient manuscripts are characterised not only by Fe but also by other metals, most commonly $\mathrm{Zn}, \mathrm{Cu}$ and $\mathrm{Pb}$ : their relative abundances represent a fingerprint of each particular ink. PIXE is an ideal technique to study these inks since it can provide all the required information without risk of damage to the paper.

This project focused on Galileo's folios dedicated to studies of the laws of motion [85-87]. The notes on experiments, statements of the properties of natural motion and calculations are simply notes and are undated.

There is a great interest in reconstructing the time evolution of Galileo's thought. In particular, it is of the utmost interest for the history of science to know when

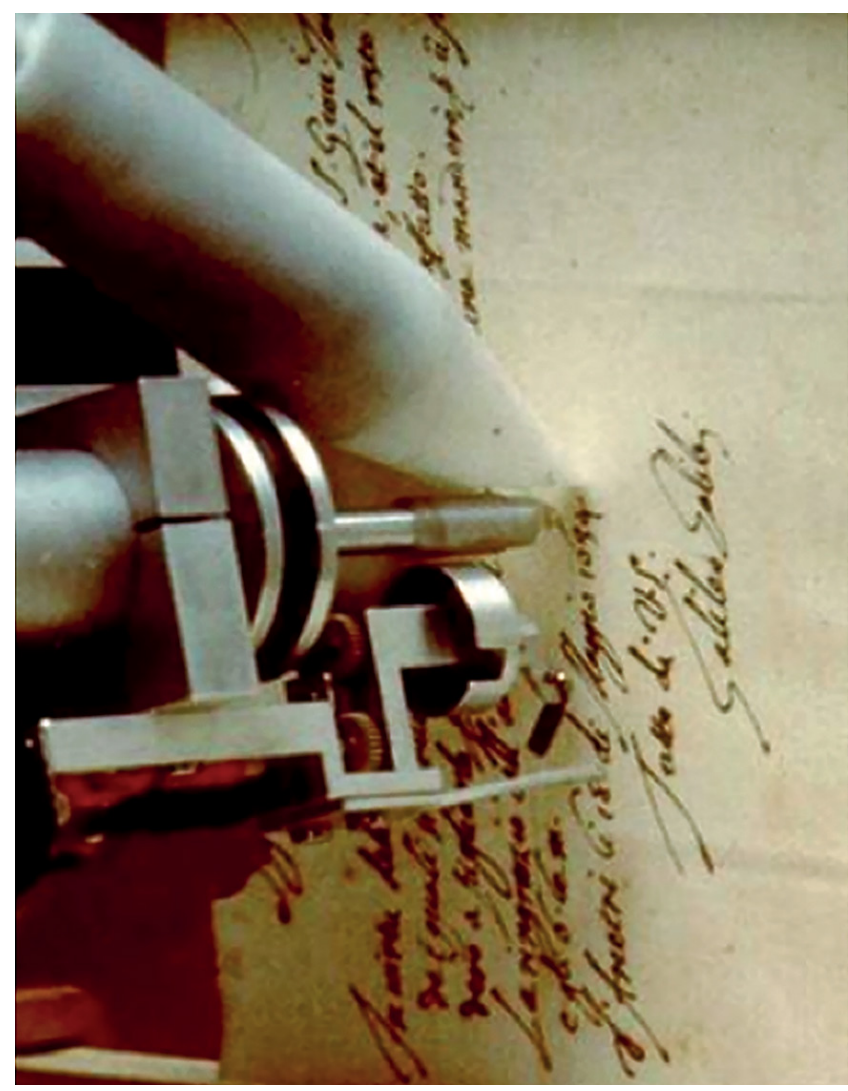

Figure 2.18: A letter signed by Galileo examined at the external beam PIXE set-up. 
Galileo began to realise that contemporary ideas regarding the motion of falling bodies were incorrect and when he arrived at a proper description of the laws of motion.

Historians of science have dedicated a great deal of effort to reconstruct the chronology of Galileo's thoughts, basing their deductions on elements such as text analysis, handwriting and ink colour. However, there continued to be much debate about the possible dating of many documents and even about the dating of single sentences.

Radiocarbon dating, the obvious choice when dating problems are concerned, is not feasible for this task. ${ }^{14} \mathrm{C}$ dates have a minimum overall uncertainty of some tens of years, whereas the resolution of uncertainties in the chronology of Galileo's thoughts, requires a time resolution of a few years. In Galileo's time, ink was hand-made by apothecaries (at the time speziali or drysalters).

This explains why ink composition is expected to vary - at least in terms of relative quantities - from one batch to another. An extensive examination of dated documents (Galileo's agenda), which covered different periods of Galileo's life, was carried out to obtain ink composition profiles.

Results obtained from dated documents indicated that the elements detected were always the same at a qualitative level $(\mathrm{S}, \mathrm{Fe}, \mathrm{Cu}, \mathrm{Zn}, \mathrm{Pb}$, and at times traces of $\mathrm{Mn}$ and light metals such as $\mathrm{Al}$ and $\mathrm{Na}$ ). However, in quantitative terms, the ratios of elements related to different periods of Galileo's life are well differentiated from each other, while these ratios remain reasonably constant within short time intervals.

These results were followed by PIXE measurements on the inks of the undated folios. Inks were analysed and the composition profiles of the undated inks were obtained. By comparing the ink compositions on the dated and undated documents, it was possible to propose a tentative chronology of Galileo's writings and to resolve issues raised by previously proposed time sequences.

Here two examples of particular interest to science historians, which deal with the laws of natural motion, are reported. In the first example, the previously proposed date was confirmed by the PIXE measurements; in the second case the previously proposed date was rejected.

\section{First example.}

Folio 128 contains a demonstration by which Galileo reaches a correct conclusion about natural motion, starting from the wrong assumption that velocities are proportional to the distance travelled, rather than the elapsed time. Science historians had dated this demonstration to the autumn of 1604 (letter to Fra Paolo Sarpi).
In folio 128 the $\mathrm{Pb} / \mathrm{Fe}$ atomic ratio is of the order of 0.5 , while in all the other examined inks it never exceeds 0.025 . In the entries dated from August 8 to December 24, 1604 (Galileo's agenda), the $\mathrm{Pb} / \mathrm{Fe}$ ratio is about 0.5 . The proposed date seems to be very reasonable!

\section{Second example.}

Arguing from an analogy with uniform motion, the second proposition on folio 164 verso states a wrong law of motion regarding bodies falling from the same height. The third proposition on folio 164 verso, on the contrary, clearly states the correct law of speeds. The second and the third propositions were supposed to come from the same period. All the considered ratios of elements $(\mathrm{Cu} / \mathrm{Fe}, \mathrm{Zn} / \mathrm{Fe}, \mathrm{Zn} / \mathrm{Cu} \mathrm{Fe} / \mathrm{Pb}$ ), measured on many points of the two sentences, look notably different, thus allowing for the rejection of the initial assumption.

\subsubsection{CORROSION OF ANCIENT GLASS}

The main advantage of the TOF "(Time of Flight)" spectrometer for ERDA is its capability to separate neighbouring light elements (starting with hydrogen) that recoil from the sample surface. Since ERDA is also a depth profiling technique it is widely utilised in material science applications. In contrast to NRA and PIGE that are generally applied to the analysis of specific light element isotopes, "TOF ERDA" is truly multi-elemental analysis technique. It is therefore very useful in studying samples that have a completely unknown composition as most cultural heritage objects are. The sampling depth is typically of the order of $500 \mathrm{~nm}$ or less, dependent on the ion mass and energy.

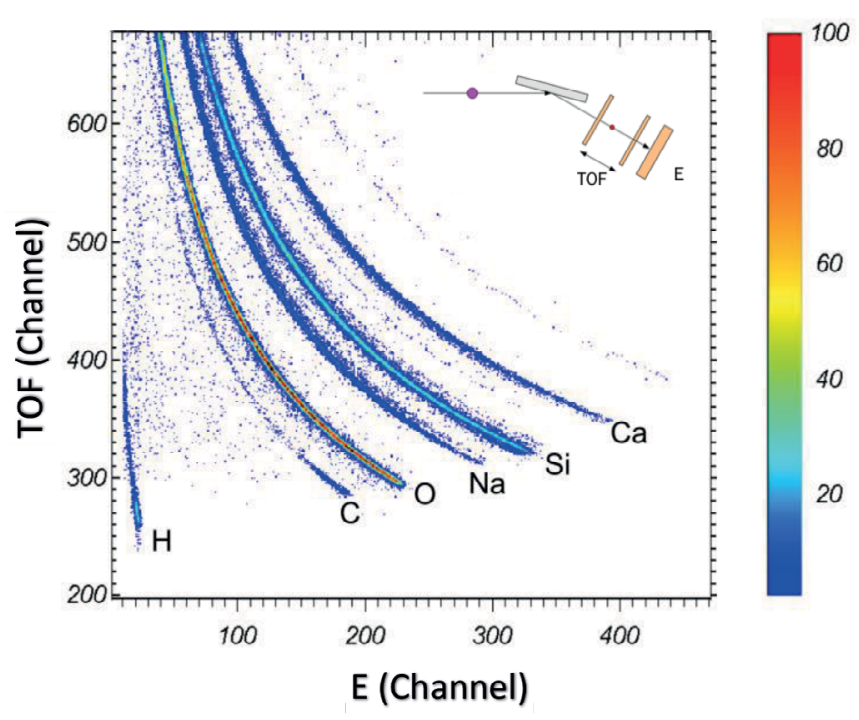

Figure 2.19: Two dimensional TOF-ERDA spectrum with schematic inset showing the experimental setup. 
In spite of the obvious advantages, ERDA can provide useful quantitative results only if the geometry of irradiation is well defined. Samples have to be flat and, if the depth profile is required, surface roughness has to be comparable to the analysis depth. Irradiation with a heavy ion beam implies a significant energy deposition, which excludes ERDA applications from materials that are sensitive to the heat induced by the ion beam.

Alloys and glasses are among the materials that are well suited to TOF-ERDA and for which there is a need to observe changes to their surfaces when exposed to the ambient atmosphere. Unfortunately, the chemical stability of glass and several metals and alloys (e.g. silver) is insufficient and the appearance of such art works can be strongly affected by being exposed to different atmospheres.

Here results of the TOF-ERDA analysis of ancient glass fragments that originate from the Sokol fort in the Dubrovnik region of Croatia are presented. These fragments come from a period between the $14^{\text {th }} \mathrm{c}$. and the $15^{\text {th }} \mathrm{c}$. While modern glass is considered stable (e.g. it is used to contain acids or even to stabilise nuclear wastes), historic glass, especially medieval glass, suffers from atmospheric pollution, predominantly in recent centuries.

As seen in Figure 2.19, where a two dimensional spectrum of the energy versus time-of-flight of detected charged particles is shown, all the major elements that form glass are clearly resolved. The spectrum was recorded using a 23 $\mathrm{MeV}$ iodine beam with the sample positioned relative to the beam at a grazing angle of $20^{\circ}$. The detection system of the TOF spectrometer at the Ruder Boskovic Institute in Zagreb is positioned at an angle of $37.5^{\circ}$ [88]. The lower edge of each individual isotope contribution in the $2 \mathrm{D}$ spectrum corresponds to the sample surface.

It is clearly seen that the $\mathrm{H}$ and $\mathrm{C}$ concentrations are more intense close to the surface, while $\mathrm{Na}$ and $\mathrm{Ca}$ concentrations decrease near the surface.

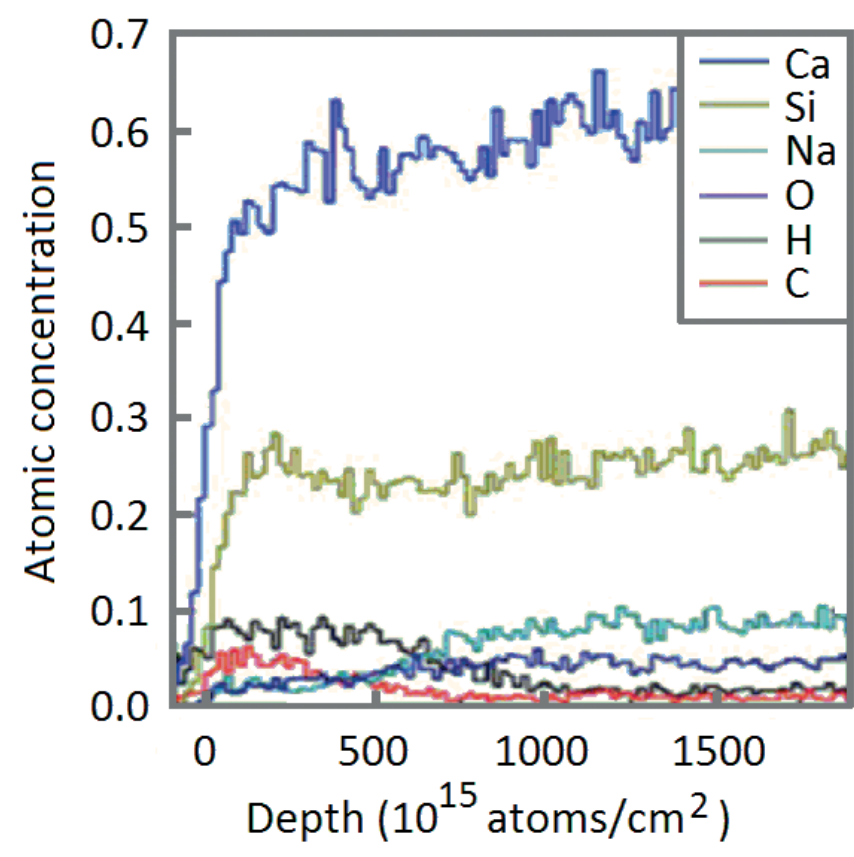

Figure 2.20: Depth profiles of selected elements in atomic concentration as a function of depth. $10^{15}$ atoms $/ \mathrm{cm}^{2}$ corresponds to approximately $0.125 \mathrm{~nm}$ of glass thickness.

As ERDA relies on pure Rutherford cross sections for the recoil particles, the accuracy of depth profiles is quite reliable. In this example the software package Potku [89] has been used. The results for the concentrations of different isotopes (in atoms $/ \mathrm{cm}^{2}$ ) are shown in Figure 2.20.

The observed glass corrosion comes from the so-called network modifiers $\left(\mathrm{Na}^{+}, \mathrm{K}^{+}, \mathrm{Ca}^{2+}\right.$, or $\left.\mathrm{Mg}^{2+}\right)$ present in the silicate structure. When water is present on the surface and the $\mathrm{pH}$ is acidic $(\mathrm{pH}<7)$ they are extracted from the glass into the solution. In order to maintain electrical neutrality in the glass, the cations are replaced by $\mathrm{H}^{+}$or other hydrogen bearing species such as $\mathrm{H}_{3} \mathrm{O}^{+}$, which is exactly what has been observed in the depth profile shown in Figure 2.20.

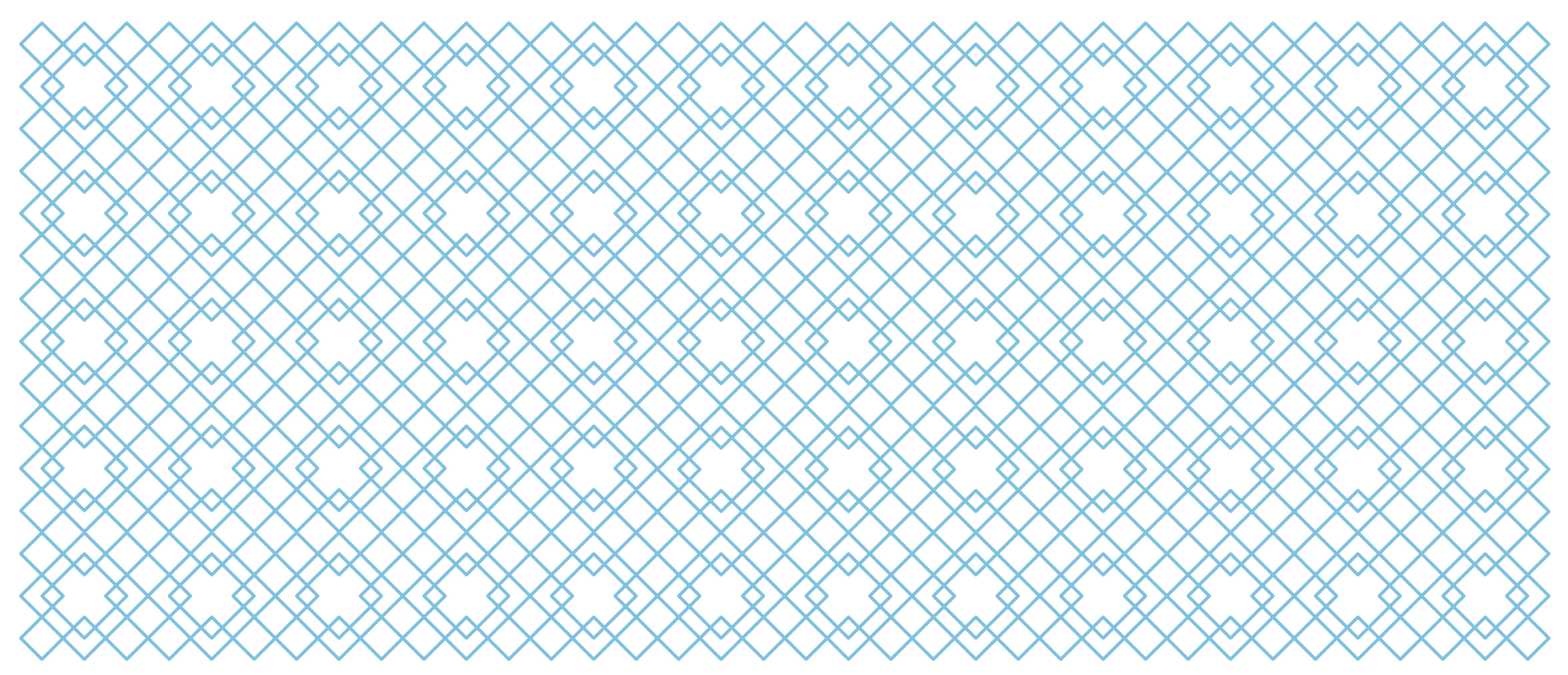




\section{NEUTRON BEAM ANALYTICAL METHODS}

$\mathrm{T}$ he neutron was discovered by James Chadwick in 1932. It is a sub-atomic particle found in the nuclei of all atoms heavier than hydrogen. It has zero electric charge and is slightly heavier than the proton. Neutrons bound inside stable nuclei are themselves stable. However, a free neutron is unstable and will $\beta$-decay into a proton, emitting an electron and an antineutrino with a half-life of 10.23 minutes.

$$
n^{0} \rightarrow p^{+}+e^{-}+\bar{v}_{e}
$$

Soon after its discovery, scientists realised that beams of free neutrons could be used as probes to explore properties of matter. Due to its zero electric charge, the neutron can penetrate deep into matter and can provide information through a variety of neutron capture or scattering interactions [90].

\subsection{BASIC PRINCIPLES OF NEUTRON BEAM ANALYSIS}

Analytical neutron techniques require sources with constant and sufficiently high neutron fluxes, and suitable neutron energies. Isotopic sources are commonly used for small-scale laboratory studies. The radioisotope ${ }^{252} \mathrm{Cf}$ produces neutrons directly in its decay. Other sources combine a-emitting radioisotopes with a ${ }^{9} \mathrm{Be}$ target. Neutrons are released when the beryllium disintegrates under a-bombardment. Common sources of this type are ${ }^{210} \mathrm{Po}-{ }^{9} \mathrm{Be},{ }^{239} \mathrm{Pu}-{ }^{9} \mathrm{Be}$ and ${ }^{241} \mathrm{Am}-{ }^{9} \mathrm{Be}$. However, the neutron flux provided by isotopic sources tends to be small.

For detailed studies scientists require much more intensive neutron fields from which neutron beams may be extracted. Such fields may be produced in nuclear reactors (also called research reactors), through the fission of nuclear fuel such as ${ }^{235} \mathrm{U}$. A constant and intense neutron flux is produced by a controlled and sustained chain reaction in the reactor. $A$ large number of research reactors have been built all over the World. Many of them (e.g. those in Garching, Delft, Saclay, Budapest and Řež near Prague) still provide very reliable and effective neutron fields for research [91].
In addition to fission reactors, neutron generators can produce $14 \mathrm{MeV}$ fast neutrons, using a beam of deuterons on a tritium target, through the ${ }^{3} \mathrm{H}(\mathrm{d}, \mathrm{n})^{4} \mathrm{He}$ reaction. Fast neutrons with varying energy in the range from 20 to 35 $\mathrm{MeV}$ can also be produced using several nuclear reactions using accelerated protons and deuterons with deuterium, beryllium and lithium targets [92].

Another large scale neutron source is the spallation source, where a high energy accelerated proton beam hits a heavy metal (e.g. Tungsten) target. As a result intense neutron radiation is emitted. The largest spallation sources used for research purposes in Europe are ISIS in the UK and SINQ in Switzerland. Both fission reactors and spallation sources are very large, expensive facilities, but they serve scientific aims in a very effective way [93].

The most common questions addressed by neutron beam analysis are the provenance of objects (i.e. the origin of their raw material), or the workshop or technique used in its production. Sometimes, it is important to know whether the object is an original or counterfeit. In many cases these questions can be answered using non-destructive analytical methods.

There is a large variety of neutron techniques, based on different types of interaction with matter, which offer a wide range of methods to explore the composition or structural features of samples. The bulk elemental composition of objects can be determined from the detection of characteristic $\gamma$-rays produced in $(n, \gamma)$ reactions. Investigation of elastic or inelastic scattering patterns can provide information on the atomic, molecular or nano-scale structural properties including their crystalline or amorphous morphology, phase composition, mechanical strains, impurities, etc. Neutrons can also be used to screen objects whose inner structure is unknown. Using neutron techniques, $2 \mathrm{D}$ or $3 \mathrm{D}$ images of objects can be reconstructed in a manner similar to X-ray radiography or tomography [94].

\section{NEUTRON ACTIVATION ANALYSIS (NAA)}

Neutron activation analysis is a multi-element analytical technique used for qualitative and quantitative analysis of major, minor, and trace elements. Samples with masses 
typically in the range from $\mathrm{mg}$ to hundreds of $\mathrm{mg}$ are irradiated with neutrons. This results in the formation of radioisotopes from isotopes of elements present in the samples, mostly via the $(n, y)$ nuclear reaction with thermal neutrons (neutron radiative capture).

The newly formed radioisotopes decay by particle emission or, more importantly, by emitting $\mathrm{Y}$-rays characteristic of the radioisotopes produced in the capture reaction. The irradiation is usually carried out in a nuclear reactor but other neutron sources (radioisotopic or accelerator based) can also be used. The neutrons used for irradiation are categorised as cold, thermal, epithermal (resonance) or fast, according to their energy.

In general, the lower the neutron energy, the higher the probability of the $(n, y)$ nuclear reaction. Detection limits are primarily determined by neutron capture cross-sections, i.e. the probability of the $(n, y)$ reaction.

Thermal neutron capture cross-sections can vary over seven orders of magnitude. Those elements with the largest neutron capture cross-sections can be measured with the lowest detection limits. These are some rare-earth elements, namely $\mathrm{Eu}, \mathrm{Dy}, \mathrm{Ho}, \mathrm{Sm}, \mathrm{Lu}$, as well as $\mathrm{Mn}$, In, Re, Ir and Au. On the other hand, elements that are hard to detect are those with low neutron capture cross-sections. These include Si, S and Fe. The element $\mathrm{Pb}$ cannot usually be determined at all, because it only forms a short-lived ${ }^{207} \mathrm{~Pb}$ radionuclide with a half-life of $0.8 \mathrm{~s}$.

The irradiation time and the time gap between irradiation and the subsequent $y$-ray measurements are chosen to optimise detection limits for the elements of interest. Commonly $y$-rays are detected and analysed using spectrometers with a High Purity Germanium (HPGe) semi-conductor detector. The evaluation of the spectra is performed offline using $Y$-ray spectroscopy computer programs.

In general, the energies of detected $y$-rays identify isotopes of particular elements in the sample and their intensities are proportional to the element concentration. The analyses are usually calibrated and quantified against appropriate element standards, irradiated and measured under the same conditions or using the $k_{0}$-standardisation process, in which neutron flux monitors are used.

This mode, which uses a purely instrumental, nondestructive, approach is commonly called instrumental neutron activation analysis (INAA). Sometimes the sample is subjected to chemical separation in which case an element of interest is selected before neutron irradiation (pre-separation NAA, PS-NAA), or a radiochemical separation of particular radioisotopes is carried out from the irradiated sample (radiochemical NAA, RNAA). In this way, it is possible to remove undesirable radionuclides and to improve substantially the detection limits for selected elements. NAA can detect up to 74 elements depending on the experimental procedure, with minimum detection limits ranging from $10^{-7} \mathrm{~g} / \mathrm{g}$ to $10^{-12} \mathrm{~g} / \mathrm{g}$, depending on the elements and matrix composition.
The NAA technique requires a small sample to be taken from the object analysed, (e.g. by drilling in an inconspicuous place) but the size of the sample is usually so small that damage to the object is minimised. Due to its high potential for accuracy and well defined theoretical background (all sources of uncertainty can be experimentally evaluated or modeled), NAA with relative standardisation has recently been recognised as a primary method of measurement, e.g. a method with the highest metrological properties.

A widespread INAA technique has been developed based on work by George Hevesy in 1936. In this case, an encapsulated sample, about $50 \mathrm{mg}$, is positioned inside the core of a research reactor and irradiated for a known period of time. Following a necessary cooling time, the so-called delayed or decay radiation is counted, usually by $\gamma$-spectrometry. Several $\gamma$-spectroscopy measurements may be performed a few minutes, days, or weeks after irradiation in order to detect the short-, medium- and long-lived products, respectively. Since the neutron flux in the reactor core is typically $10^{12}-10^{14} \mathrm{~cm}^{-2} \mathrm{~s}^{-1}$, the method is very sensitive for a series of trace elements. However, because of the high neutron flux, there is a risk of significant radiation damage and of producing nuclides with long half-lives in the sample [95].

\section{PROMPT GAMMA ACTIVATION ANALYSIS}

A slightly different technique is prompt gamma activation analysis (PGAA or PGNAA). In this case even large-sized samples can be placed in a guided neutron beam extracted from a reactor. Due to their low energy and relatively low intensity $\left(10^{7}-10^{9} \mathrm{~cm}^{-2} \mathrm{~s}^{-1}\right)$ guided neutron beams are perfect tools to study valuable and irreplaceable cultural heritage objects and artefacts, where destructive or invasive methods are out of the question. Generally neutron investigations cause no observable damage to artefacts and most of the induced radioactivity decays within a few days.

When the sample is irradiated, both the prompt and some delayed $y$-rays are detected during the irradiation. Since every chemical element, except He, emits prompt $\mathrm{Y}$-rays, the method can in principle detect all elements in the periodic table, but with very different sensitivities. The most sensitive elements include B, Cd, Nd, Sm, Eu and $\mathrm{Gd}$, whereas the least sensitive elements are $\mathrm{Be}, \mathrm{C}$, $\mathrm{N}, \mathrm{O}, \mathrm{F}, \mathrm{Pb}$ and $\mathrm{Bi}$. Since the number of prompt $\mathrm{Y}$-rays is much higher compared to decay $\gamma$-rays, the prompt $y$-ray spectrum is generally much more complicated than the INAA spectrum. In both instances the identification of chemical elements and their quantitative analysis is based on the precise determination of energies and intensities of $\mathrm{Y}$-rays.

The first applications of PGAA were performed in Saclay and Grenoble in the late 1960s and early 1970s. This method has become more widely used since the 1980s, 


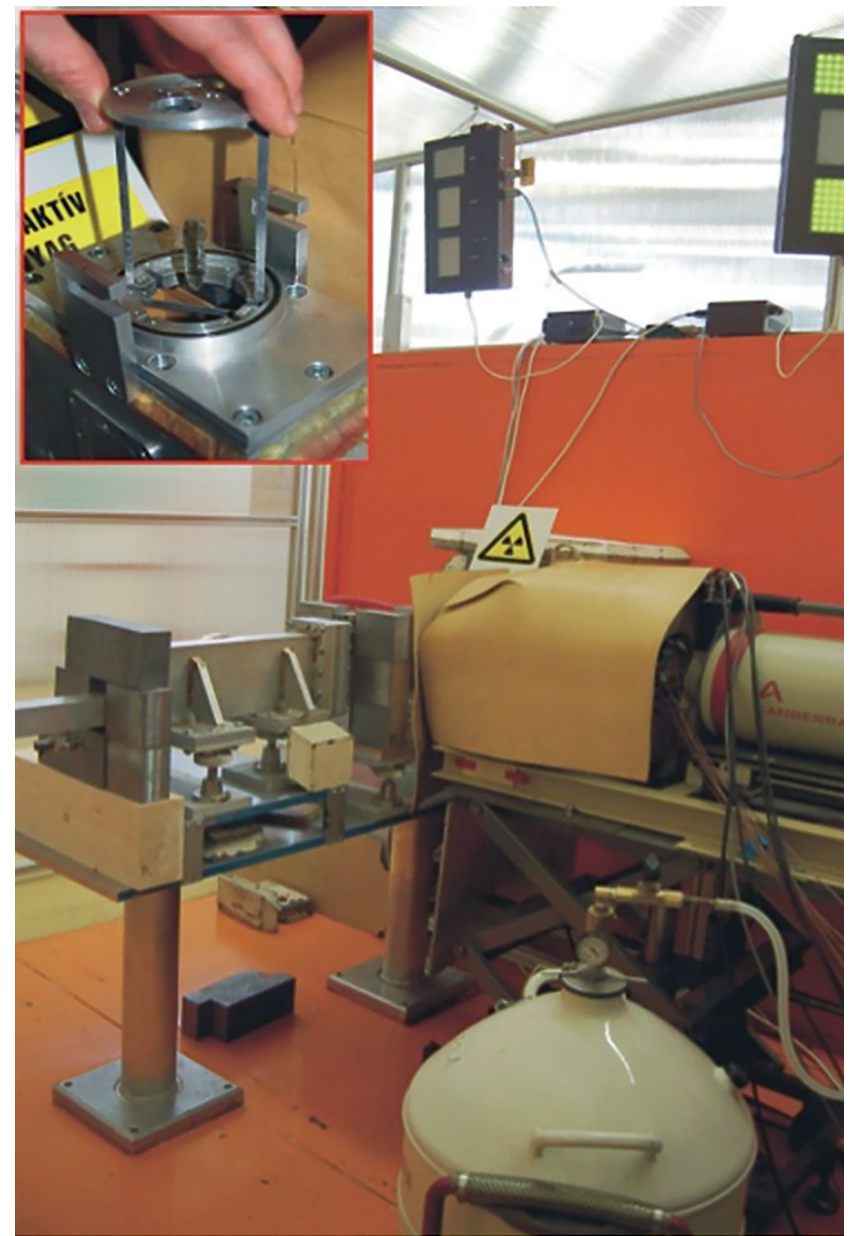

Figure 3.1: The Prompt Gamma Activation Analysis facility at the Budapest Neutron Centre. Insert photograph: A sample holder with a small obsidian sample.

when high intensity neutron guides with super mirrors were built next to research reactors and high resolution HPGe detectors became widely available. See Figure 3.1.

INAA induces at least some minimal damage to objects, but is very sensitive for trace element analysis. In contrast, intact objects remain after PGAA with almost no induced radioactivity, because of the lower $\left(10^{7}-10^{9} \mathrm{~cm}^{-2} \mathrm{~s}^{-1}\right)$ neutron flux in the beam.

Thus PGAA is more sensitive to major components and provides information on only a few trace elements with high neutron absorption cross sections $(\mathrm{H}, \mathrm{B}, \mathrm{Cl}, \mathrm{Cd}$ and rare-earth elements). For this reason, these two standard neutron activation techniques are complementary.

\section{NEUTRON DEPTH PROFILING (NDP)}

Neutron Depth Profiling is a prompt analysis technique which uses neutron-induced nuclear reactions accompanied by the emission of charged particles with a specific kinetic energy [96-98]. It is one of the most powerful nondestructive techniques for depth profiling of particular light elements, especially ${ }^{10} \mathrm{~B}$ and ${ }^{6} \mathrm{Li}$, which have very high thermal neutron capture cross-sections. The samples are placed in a thermal neutron beam (usually from a nuclear reactor) and the energy spectra of emitted charged particles are measured using semiconductor detectors (see figure 3.2 top). NDP is extensively used for the determination and depth profiling of ${ }^{3} \mathrm{He}\left(10^{-3}\right)$, ${ }^{6} \mathrm{Li}\left(10^{-3}\right),{ }^{10} \mathrm{~B}\left(10^{-2}\right),{ }^{14} \mathrm{~N}(1)$, and ${ }^{35} \mathrm{Cl}(10)$. The numbers in parentheses are typical detection limits in units of $10^{15}$ atoms $/ \mathrm{cm}^{2}$.

If the neutron-capture reaction takes place beneath the sample surface, the energy loss of the charged particles emerging from the sample can be used to obtain information about the element concentration profile as a function of depth (see figure 3.2 bottom). The magnitude of the energy loss is simply related to the distance that the charged particle has travelled within the specimen.
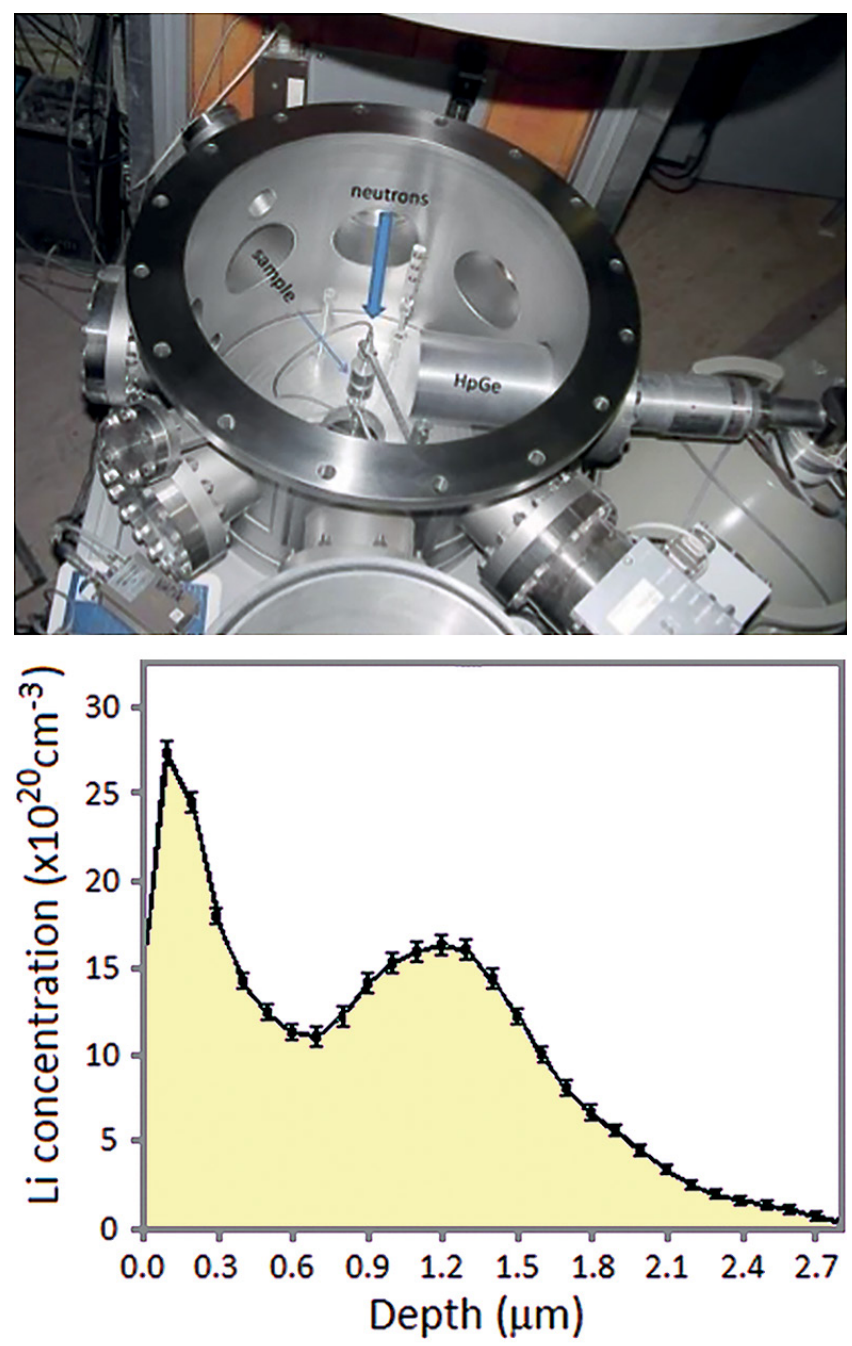

Figure 3.2: Top: Vacuum chamber for NDP analyses used by CANAM NPI at the nuclear reactor (Research Centre Řež Ltd.). Bottom: Depth profile of lithium atoms in tantalum determined from measurements of the energy spectrum of ${ }^{3} \mathrm{H}$ and ${ }^{4} \mathrm{He}$ particles emitted in the reaction ${ }^{6} \mathrm{Li}+\mathrm{n}$. The sample was implanted with $300 \mathrm{keV} \mathrm{Li}$ ions and annealed at $1800^{\circ} \mathrm{C}$. [97] 


\subsection{INSTRUMENTATION OF NEUTRON BEAMS}

Neutron beams are perfect tools for archaeometric studies. Various neutron techniques offer a wide range of methods of exploring the compositional or structural features of samples. Due to the low energy and relatively low intensity $\left(10^{7}-10^{9} \mathrm{~cm}^{-2} \mathrm{~s}^{-1}\right)$ of guided neutron beams, no damage to objects is observed, and the induced radioactivity decays within a few days.

Research reactors with compact cores, or acceleratorbased spallation sources, provide intense neutron beams for experiments. These are usually taken off from cold or thermal moderators by sophisticated neutron optical systems. These neutron beam transport devices are known as neutron guides - they provide collimated, highly parallel beams of thermal and cold neutrons for measuring instruments and spectrometers.

Based on different types of interactions of neutrons with matter, capture or scattering reactions can be used. For example, by detecting characteristic $y$-rays produced in $(n, y)$ reactions, the bulk elemental composition of objects can be determined. These techniques (NAA, PGAA, NDP) are described in detail in the previous section. On the other hand, through investigation of elastic or inelastic scattering patterns, information can be obtained on the atomic, molecular or nano-scale structural properties, including information on crystalline/ amorphous morphology, phase composition, mechanical strains, and impurities. The most important techniques and instrumentation relevant to investigations of cultural heritage objects are now described.

Neutron diffraction or elastic neutron scattering is the application of neutron scattering to the determination of the atomic or magnetic structure of a material. The technique is similar to $X$-ray diffraction but due to their different scattering properties, neutrons and X-rays provide complementary information: $X$-Rays are suited for superficial analysis; strong $x$-rays from synchrotron radiation are suited for shallow depths or thin specimens; neutrons, which have a high penetration depth, are suited for bulk samples.

Neutron diffraction is most commonly performed as powder diffraction, which only requires a polycrystalline powder or amorphous sample. The incoming neutron beam needs to be mono-chromatised, to sort the energies of the incident neutrons (higher energy neutrons are faster). This can be done using a single crystal monochromator. The time-of-flight (TOF) technique can also be used. No monochromator is needed in the TOF technique. Rather, a series of aperture elements (choppers) are synchronized to filter neutron pulses with the desired wavelength (energy). Neutrons coming from the sample are detected in counters to measure the angular distribution of the scattered neutrons. A typical example of such a diffractometer is in use at the Budapest Research Reactor.
This unique type of TOF powder diffractometer is used on a continuous beam chopped to produce several welldefined wavelength bands, which vary periodically within a selected wavelength range. This type of instrument usually outperforms a conventional crystal monochromator powder diffractometer and has increased resolution in atomic position determination. See Figure 3.3.

This TOF instrument plays an essential role in exploring the material properties of archaeological objects, e.g. crystalographic phase analysis can reveal ancient technologies. Differences in the diffraction patterns are usually due to different compositional and/or treatment features.

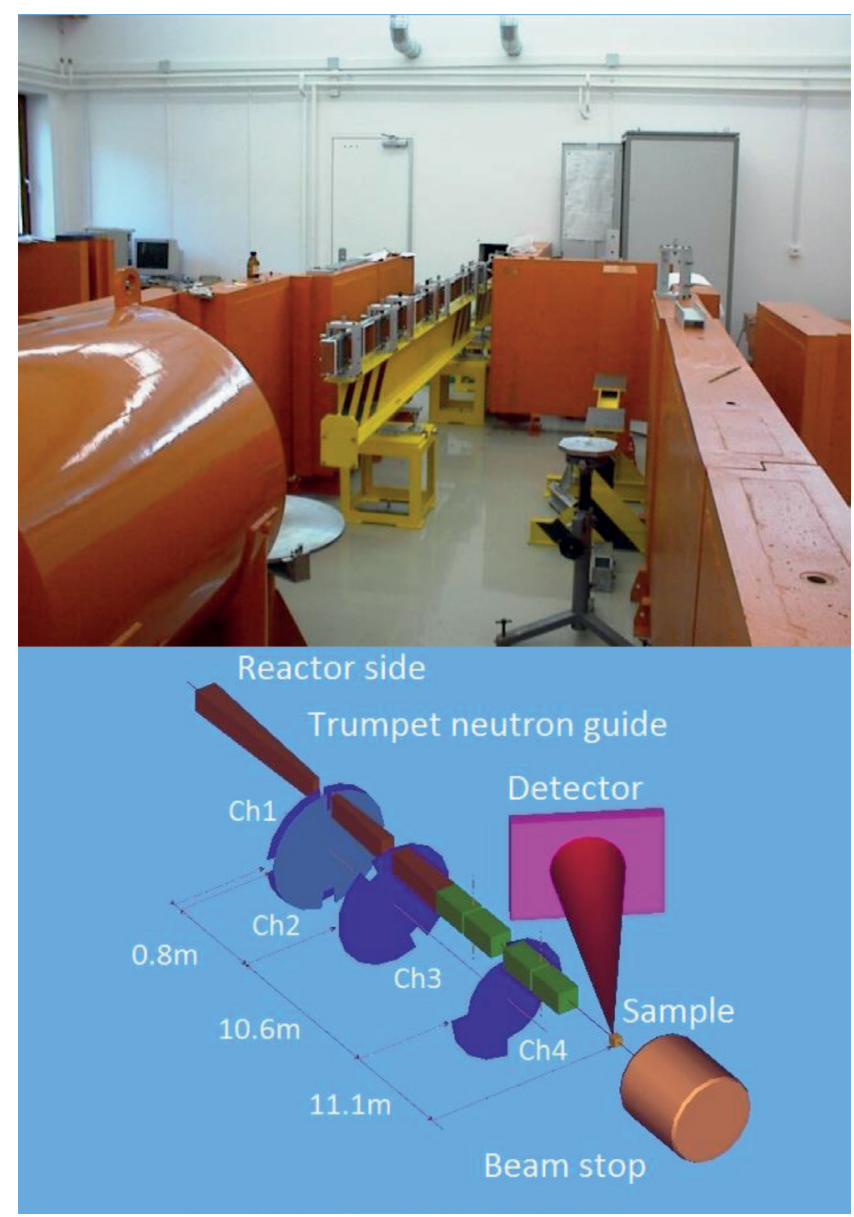

Figure 3.3: Photograph and Schematic illustration of the neutron TOF instrument set-up showing the real neutron guide together with its shielding components.

A typical application of this instrument is in the study of lapis lazuli, a beautiful light blue stone, which was a high grade gemstone and pigment in the Near-East, Egypt, and later in Europe. Its geological occurrences are well known and each have particular differences in their detailed chemical composition.

There were already attempts in antiquity to produce artificial lapis lazuli, such as Egyptian Blue, i.e. $\mathrm{CaCuSi}_{4} \mathrm{O}_{10}$. Both PGAA and time-of-flight neutron diffraction TOF-ND are now capable of distinguishing between false and true lapis lazuli [94]. 


\section{SMALL ANGLE NEUTRON SCATTERING (SANS)}

SANS is a technique for studying nanometer scale structural features in materials. The information obtained, however, is characteristic of the whole irradiated volume of the sample.

Practically, the measured piece is free from deformation, physical, chemical or structural changes and there is no need for sampling.

The SANS technique can determine void sizes in porous media such as cements and marble. It can also identify anisotropies in precipitate orientation in minerals or metals and facilitates the investigation of particle agglomeration in ceramic bodies and the evolution of pores during different types of processing.

In the majority of SANS experiments the scattering of neutrons is isotropic; the studied particles can be regarded as spheres. So far anisotropic SANS experiments were mostly used to study the shape and orientation of defect agglomeration in single crystals based on the asymptotic behaviour of the scattering pattern. However 2D data analysis software has been developed and successfully used for non-isotropic SANS data.

The Yellow Submarine SANS spectrometer, operating at the cold neutron source of the Budapest Research Reactor, is a large-scale instrument that covers a study range of material inhomogeneities from $50 \AA$ to $1500 \AA$.

SANS can be a proper tool for investigation of structural differences between marble, ceramic, metal objects of different provenance or technologies.
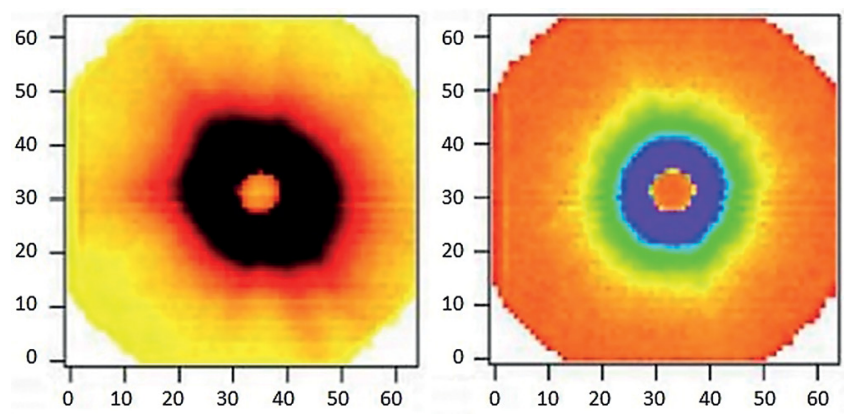

Figure 3.4: Comparison betwen two 2D neutron scattering patterns, measured using a $64 \times 64$ pixel position sensitive neutron detector, from two different marble samples.

A study on archaeological marble samples is presented in Figure 3.4. The left sample shows a strong anisotropy, which is caused by the morphology of the marble as a consequence of its geological history, while the right sample is isotropic.

\section{NEUTRON DEPTH PROFILING (NDP)}

Samples are irradiated with thermal neutrons and the energy spectra of charged particles are measured by semiconductor detectors (PIPS, PINs) connected to standard electronic devices for data acquisition [99]. The samples are irradiated in a vacuum chamber placed on external neutron beam from a nuclear reactor. The neutron beam is usually transported to the chamber via a neutron guide, transparent for thermal neutrons. The size of the neutron beam can be limited by collimators of neutron absorbing materials. The beam size can further be reduced, down to micrometer size, by neutron lenses constructed from glass capillaries.

\subsection{APPLICATIONS OF NEUTRON BEAMS}

The great advantage of neutron-based methods in researching Cultural Heritage objects is that the majority of techniques are completely nondestructive and non-invasive, although some may require a small sample to be extracted discretely from the object. Generally this means that no visible modifications are observed following investigations. Information on both the composition and structure can be obtained from priceless artefacts made of rocks, metals, ceramics, glass, etc. The combination of neutronbased techniques with other methods, such as PIXE, can provide even more comprehensive and valuable information.

\subsubsection{PROVENANCE OF PREHISTORIC STONE TOOLS}

In prehistoric times people knew where good quality materials could be quarried to make everyday tools. Sometimes, final or semi-final products were carried hundreds of kilometres from the localities where the raw materials were mined. If we can analyse the composition of tools and fingerprint chemical components characteristic of the material's provenance, this can enormously help archaeologists to reconstruct prehistoric trade and migration routes.

Non-destructive studies can also help distinguish between different basic types of raw materials (e.g. obsidian, flint, silex, felsitic porphyry) which are sometimes easy to confuse on the basis of visual examination [100]. In the Budapest PGAA laboratory, studies have found that knowledge of the bulk elemental composition can differentiate between obsidian, silex and felsitic porphyry.

The easiest task is to determine the provenance of obsidian - a volcanic glass which was popular as a raw material from the early Palaeolithic period. See Figure 3.5. The geological sources are quite well known and their compositions are distinctive of the geological formation and thus define the locality. Besides trace elements of $R b$, $\mathrm{Nb}, \mathrm{Yb}$, etc. that can be measured by destructive INAA, B and $\mathrm{Cl}$, which are both easy to measure non-destructively by PGAA, were found to be fingerprints as well. 
Using a PGAA obsidian database, a better overview of the distribution of obsidian material in Central Europe has been obtained. In particular a border zone between the distribution areas of the so-called Carpathian and Lipari obsidians has been identified. This border falls in the inland part of modern Croatia [101].

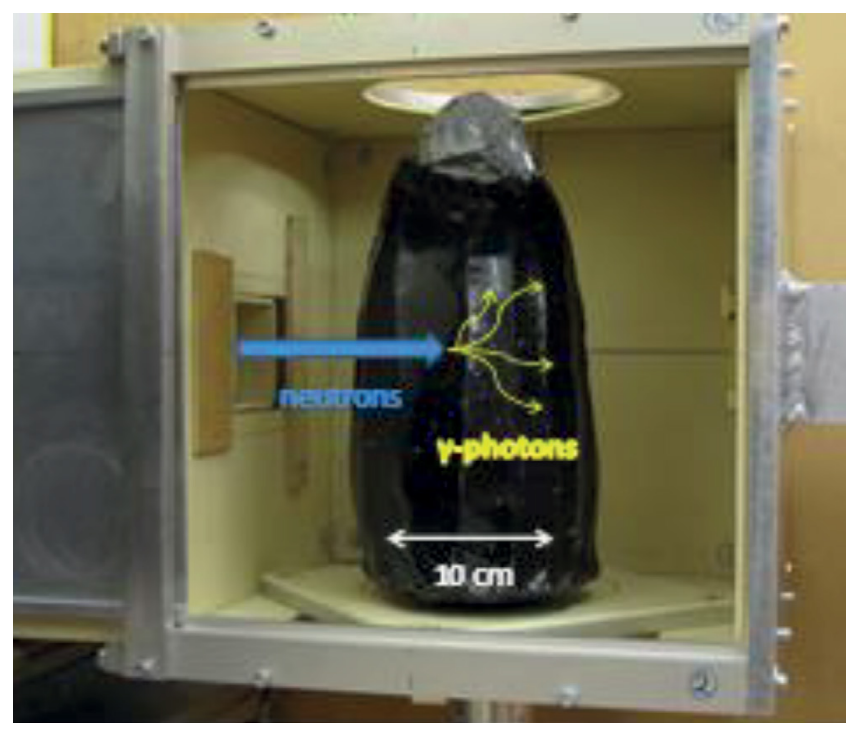

Figure 3.5: An obsidian core from Nyirlugos, Hungary held in the sample holder of the NIPS (PGAA) station of the Budapest Neutron Centre. The object is from the collection of the Hungarian National Museum.

In cases where it is allowed to take samples from the objects, INAA could help to quantify additional trace elements. With an extended database including both major and trace components, one can try to perform provenance analysis. In the investigation of stone objects the PGAA method has a great advantage when it is necessary to determine the average bulk composition of a rock, without sampling any unique objects.

\subsubsection{TRACING ANCIENT POTTERY}

From Neolithic times, ceramics have formed the most abundant group of archaeological discoveries. Various styles, production techniques and workshops can be identified on the basis of typology, as well as using analytical methods. The results can provide information about the exchange of goods and the spread of techniques, etc.

Since ceramic is a composite material consisting of clay, temper and sometimes glaze or paint layers on the surface, one has to be careful choosing the most appropriate method and properly interpreting the results.

From the chemical composition of clay, one can identify deposits where the raw material might originate. Major clay components can be determined using PGAA (in fact an average composition is determined for just a few $\mathrm{cm}^{3}$ ). Characteristic trace elements can be determined with the help of INAA or X-rays. Further microscopic studies of thin sections can help to identify workshops or cultures.
A project lead by the Simon Bolivar University in Caracas, Venezuela, aimed to identify the raw material used to make pre-Columbian $\left(13^{\text {th }}-15^{\text {th }}\right.$ c. $\left.A D\right)$ pottery figurines made in the Valencia Lake Basin region [102]. Fragments from the continental (Valencia Lake Basin) as well as from the nearby Caribbean islands (Los Roques Archipelago) have been analysed by PGAA and by INAA. As a result we have found, with high confidence, that inhabitants of the Los Roques Archipelago used local clay to produce the figurines instead of importing the raw material from the continent. See Figure 3.6.

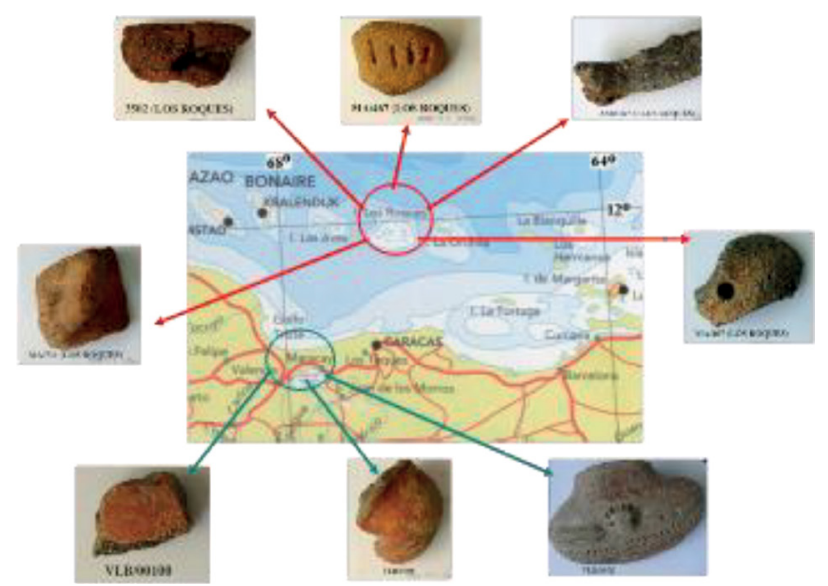

Figure 3.6: Origin of pre-Columbian pottery fragments studied by PGAA at the Budapest Neutron Centre.

PGAA and NAA have been applied in many research projects on archaeological ceramics from Europe [103], as well as from the New World [102,104]. Besides the chemical composition, the structure of ceramic materials on a micro or nanoscale may deliver information about the characteristics of production (fineness of clay, firing temperatures, etc.). For this purposes, TOF-ND and Small Angle Neutron Scattering (SANS) can be used [105].

\subsubsection{DEVALUATION OF NOBLE METALS}

Silver and gold have been some of the most valuable materials in history from ancient Egypt ( $3^{\text {rd }}$ millennium B.C.) up to present times. Since their value can be considered relatively constant with time, silver and gold functioned as currency. The production of silver from silver-bearing minerals (argentite $-\mathrm{Ag}_{2} \mathrm{~S}$ or galenite - PbS with $1 \%$ $\mathrm{Ag}_{2} \mathrm{~S}$ ) was first carried out in ancient Anatolia. However, during the time of the Roman Empire, the values of coins were intentionally modified by different Emperors. In the course of an economic crisis, the silver content of coins was gradually reduced. Interestingly, this was done without any visible sign on the coin surfaces.

Thanks to non-invasive PGAA, it was possible to show that the total silver content of later coins were significantly lower. On the other hand, it was impossible to detect this tendency, either by visual observation or by surface sensitive XRF analysis [106]. 


\subsubsection{METEORIC JEWELLERY}

A study combining various neutron beam methods has revealed that 5000 year old Egyptian iron beads have been found to be made from hammered pieces of meteorites [107]. The study focuses on the earliest known iron artefacts - nine small beads securely dated to circa 3200 BC, from two burials in Gerzeh, northern Egypt. The iron beads were strung into a necklace together with other exotic minerals such as lapis lazuli, gold and carnelian, revealing the status of meteoritic iron as a special material on a par with precious metal and gem stones. See Figures 3.7 and 3.8.

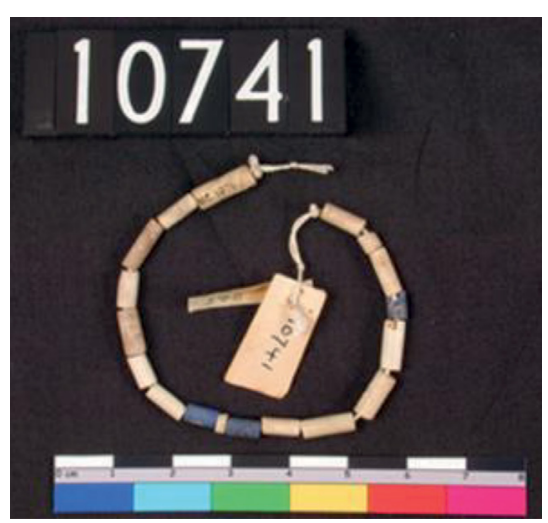

Figure 3.7:

Collection of

stone and faience beads from Tomb 67. Modern restringing, without the iron beads.

Figure 3.8: Neutron radiographs of the three beads 10740 , 10739, 10738 (from top), in side view and longitudinal.
The experimental study of three iron beads was performed at the Budapest Neutron Centre. Neutron and proton beam techniques were applied: PGAA, TOF neutron diffraction and PIXE measurements provided compositional data showing that the beads were made from meteoritic iron.
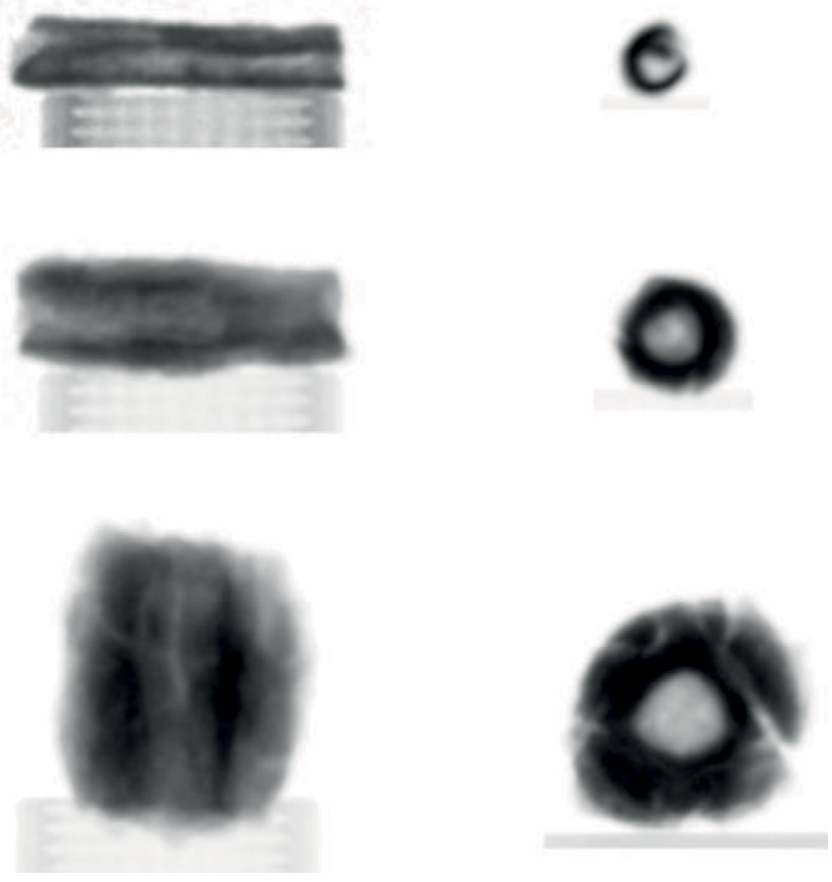

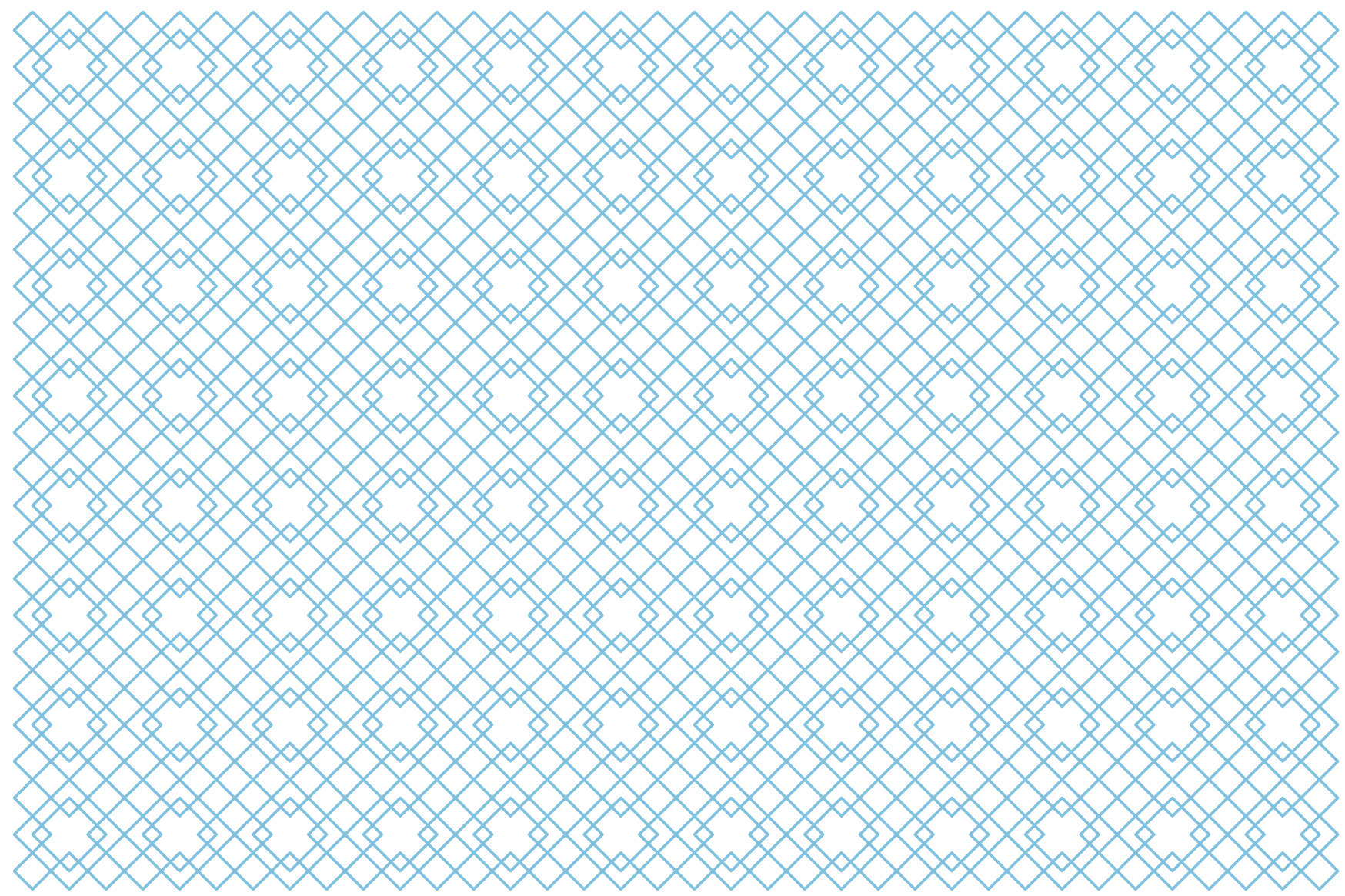




\section{DATING METHODS - LUMINESCENT DATING AND ACCELERATOR MASS SPECTROMETRY}

\subsection{BASIC PRINCIPLES OF DATING METHODS}

Radiocarbon dating provided by Accelerator Mass Spectrometry (AMS) has proved to be one of the most useful dating tools for many groups of scientists: archaeological, environmental and geological studies all benefit from the possibility of dating organic materials directly related to events under study.

Luminescence dating is an absolute dating method suitable for determining the age of archaeological ceramics as well as the age of sediment layers at archaeological sites. It relies on natural ionising radiation and the luminescence properties of minerals.

\section{LUMINESCENCE DATING}

Traces of natural radioisotopes are present in all materials, so objects are exposed continuously to their own radiation as well as to radiation from their environment. In steady state conditions the energy of this ionising radiation is absorbed in matter at a fixed rate $(d)$, so the total absorbed dose $\left(D_{L}\right)$ is specified by the so-called age equation [108]:

$$
D_{L}=d \times t .
$$

In luminescence dating the dose-rate value $d$ can be estimated by measurements of the radioactivity of natural radio-isotopes or by using in situ dosimetric methods. The total absorbed dose can be determined by a measurement of the luminescence of the material under study. Hence the age of the material $(t)$ can be determined.
Ionising radiation generates free carriers (electrons and holes) in minerals present in ceramic objects. These free carriers continuously undergo relaxation, but a small fraction can become trapped in long-lived metastable states, related to specific defects in the crystal lattice. If kept free of stimulating factors such as light or heat, the carriers can remain in these states for hundreds of thousands of years, depending on the features of the trapping states. The longer free carriers are generated by radiation the higher the total number of trapped carriers. Therefore this number can serve as a measure of the total accumulated dose $D_{L}$. The trapped electrons or holes are counted when they are freed using an external stimulus, and then relax by photon emission.

The best known kinds of stimulated luminescence are thermoluminescence $(T L)$, where the external stimulus is heating, and optically stimulated luminescence (OSL) where the stimulation factor is light of selected wavelengths. The stimulating factor, either heating or light, releases the carriers from the traps. Following efficient stimulation, the luminescence decays and subsequent stimulation will not cause any further luminescence until the mineral grains are again exposed to ionising radiation.

This means that any initial luminescence due to the radiation dose absorbed by the minerals before the creation of a ceramic object is erased during the firing process. The same considerations apply to the luminescence of minerals from a sediment layer that was exposed to sunlight before it was covered by another layer.

The annual dose absorbed by mineral grains extracted from an object of interest is generally determined by one of two distinct dosimetric methods. A direct method makes use of highly sensitive commercial TL or OSL dosimeters to determine the external dose rate due to y radiation. A set of TL or OSL pellets is placed in the location from which the sample was collected and left there for periods of up to a year. Alternative indirect methods, which rely on measurements of the concentrations of 
radioactive isotopes such as ${ }^{238} \mathrm{U},{ }^{232} \mathrm{Th}$ and ${ }^{40} \mathrm{~K}$ in the sample and its surroundings, are more common [109]. The effective dose-rate values in these indirect methods are generally obtained by applying tabulated conversion factors [110].

Regardless of which type of dosimetry method is used to determine the annual dose, factors such as the radioactivity distribution within the sample and its environment, water content, cosmic radiation and sensitivity to a radiation also have to be taken into account.

In the simplest case radioactivity is distributed uniformly within a sample. However, in practice the situation is often more complex. For instance, when large diameter grains are used for luminescence measurements the distribution is likely to be non-uniform because of the specific radioactivity of different minerals.

Feldspar (especially potassium feldspar) contains considerable amounts of ${ }^{40} \mathrm{~K}$, while quartz is commonly free of any traces of radioactivity.

In general, the range of radiation within grains must be taken into account. The ranges of a-particles are so small that removing the outer layer of grains by etching allows the a dose-rate in coarse quartz grains to be neglected. The maximum ranges of $\beta$ particles are about $2 \mathrm{~mm}$. For most objects it is necessary to use attenuation factors in order to account for the non-uniformity of the absorbed $\beta$ dose within the grain volume. However, in the case of ceramic shards, removing a $2 \mathrm{~mm}$ thick surface layer is sufficient to allow the external $\beta$ dose to be ignored. The mean $y$ range for natural radiation is typically around $30 \mathrm{~cm}$. This means that a particular sample absorbs $y$-radiation originating from within a 50-60 cm diameter sphere and the non-homogeneity of the surroundings in such a relatively large volume has to be taken into account. In more complex cases the annual dose assessment may need computer simulations of the transport of radiation and its interaction with matter [110].

\section{AMS DATING}

When a living organism dies, it stops incorporating carbon, and its existing ${ }^{14} \mathrm{C}$ decays at a known rate because it is radioactive. Thus, the ${ }^{14} \mathrm{C}$ concentration decreases with time. As the decay rate is well known, measuring the proportion of ${ }^{14} \mathrm{C}$ in organic materials provides the information necessary to calculate the time since the organism died. This is the basic principle of radiocarbon dating.

The basic principles of radiocarbon dating have been well studied and understood since the method was proposed by W.F. Libby in the 1950s [111]. Briefly, ${ }^{14} \mathrm{C}$ is formed in the atmosphere through the interaction of cosmic rays with ${ }^{14} \mathrm{~N}$, the most abundant nitrogen isotope. The ${ }^{14} \mathrm{C}$ oxidises to $\mathrm{CO}_{2}$ and enters the food chain via photosynthesis or dilution in the oceans.
Due to the continuous exchange of carbon between living organisms, the whole biosphere is in a dynamic equilibrium and the organisms contain a small but fixed proportion of ${ }^{14} \mathrm{C}$ (typically one carbon atom in $10^{12}$ ). The two stable isotopes, ${ }^{12} \mathrm{C}$ and ${ }^{13} \mathrm{C}$ represent $98.9 \%$ and $1.1 \%$ of the total, respectively. Of course there are several details that make the whole process more complex, but fortunately there is a high level of understanding of these issues which are taken into account by radiocarbon practitioners.

A major issue in radiocarbon dating is how best to detect radiocarbon atoms. Initially radiometric techniques were employed, which detected radioactive isotopes by analysing the radiation they emit. However, in the late 1970 s a new ultrasensitive tool: Accelerator Mass Spectrometry (AMS) was developed. This is now the most widely used technique to determine radiocarbon concentrations.

\subsection{INSTRUMENTATION OF DATING METHODS}

\section{LUMINESCENCE DATING}

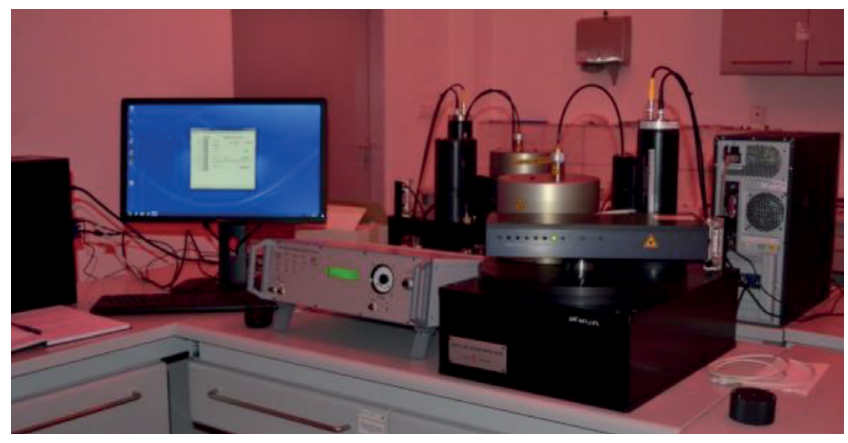

Figure 4.1: Equipment used to measure TL (thermoluminescence) and OSL (optically stimulated luminescence) in a dating laboratory.

The maximum age of an object which can be investigated using luminescence dating is determined by two factors - the concentration of trapping states in the material and the level of radioactivity it is subjected to, either from its own internal radioactivity or from its surroundings

The total absorbed dose $\left(D_{L}\right)$ of an object can be determined by luminescence measurements on grains extracted from the sample. In recent times, optically stimulated luminescence is the most common method used for this purpose [112-114]. To improve the precision in measuring $D_{L}$ a series of measurements using many aliquots, or many grains, may be used. Normally, the average precision achieved is about $2 \%$. However, this value can be reduced to $0.5 \%$ when very sensitive grains or objects are investigated.

The equipment used for luminescence dating measurements is commercially available. The systems on 
offer (see Figure 4.1) allow very low luminescence signals to be detected. They also include the radiation sources necessary for the laboratory excitation of luminescence and provide standard automated procedures to measure large numbers of aliquots or grains.

The nature of stimulated luminescence and natural radiation imposes particular requirements on the objects selected for dating. The zeroing of the luminescence signal arising prior to the object creation is a fundamental requirement. It limits acceptable materials to those that were heated to over $500{ }^{\circ} \mathrm{C}$ (e.g. any kind of ceramics, stones from fireplaces etc.) or exposed to light over all, or a significant part, of their volume prior to burial (e.g. sediment layers or buried pieces of glass). The time determined by the dating procedure is then the time since this zeroing process.

When carriers have filled all the available traps in the material, further radiation exposure will not produce any additional increase in the luminescence signal. On the other hand, the higher the level of radioactivity the faster the carriers will fill up the traps and the earlier the luminescence signal will saturate. Therefore there is a general, approximately known, age limit for the luminescence dating of a given material. In the case of quartz grains this limit is around 300,000 years, whereas $1,000,000$ years is possible for potassium feldspar. Such time ranges significantly surpass the oldest objects related to human activity.

Another consideration is the stability of the radioactivity background of the sample throughout the dating period. The moisture content and thickness of the soil layer covering an object influences its annual dose. One should not therefore attempt to date relics for which changes in these factors could be significant and for which the range of fluctuation is unknown.

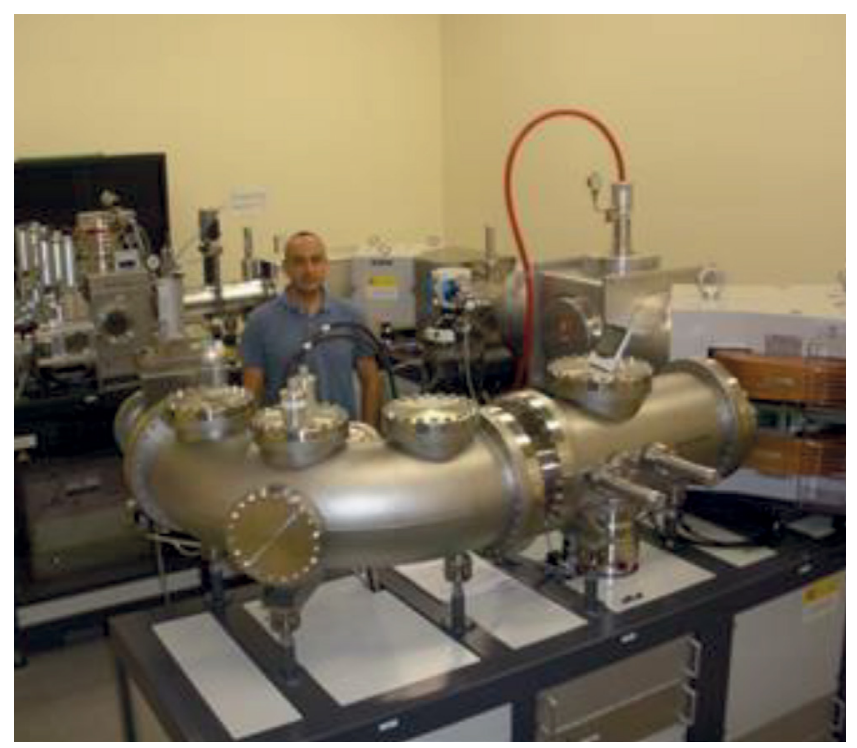

Figure 4.2: Modern compact AMS facility at CNA Seville, Spain [115].

\section{AMS INSTRUMENTATION}

The AMS technique does not use the radioactive character of ${ }^{14} \mathrm{C}$ for detection. Instead, it uses microscopic differences in isotopic masses to separate ${ }^{12} \mathrm{C},{ }^{13} \mathrm{C}$ and ${ }^{14} \mathrm{C}$.

In this process, a solid graphite sample, previously prepared in the laboratory, is placed in an ion source and carbon ions are extracted as a beam. The beam passes through a magnetic field where different isotopes have different trajectories, depending on their masses and charges. This way, ${ }^{14} \mathrm{C}$ ions can be selected. AMS is a very sensitive technique in which the beam is accelerated, using a particle accelerator, to much higher energies than available in standard mass spectrometric techniques, before passing through one or more mass analysers. See Figure 4.2.

AMS is a very valuable technique for radiocarbon dating which can identify one ${ }^{14} \mathrm{C}$ atom in $10^{15}$ carbon atoms. The process uses a very small quantity of material extracted from the original sample. Labs usually prepare AMS samples from milligrams, or even micrograms, of material. Typical measurement times are less than an hour. The laboratory can be as small as a few square meters and good results are obtained with accelerating voltages as low as $200 \mathrm{kV}$.

Negative ions from the source are initially attracted to a positive voltage terminal and then during the acceleration stage, the beam passes through a stripper channel, filled with gas. Here the accelerated ions collide with gas atoms. In the stripping process the negative ions lose electrons, become positively charged, and are repelled by the positive voltage, gaining even more energy. In addition, molecules which happen to have the same mass as the ion of interest, and which would otherwise pass the mass filters, split up, and are eliminated in subsequent filters. At the end of the beam line a detector identifies and counts events recognized as ${ }^{14} \mathrm{C}$. At the same time, the stable ${ }^{12} \mathrm{C}$ and ${ }^{13} \mathrm{C}$ isotopes are also measured in the system.

The first AMS facilities were very large instruments, accelerating ions through several million volts. Nowadays, AMS systems are small and compact, simpler to use, and are extremely effective in detecting radiocarbon ions. Modern facilities are able to measure directly over gaseous $\mathrm{CO}_{2}$ samples, avoiding the graphitisation process. 


\subsection{APPLICATIONS OF DATING METHODS}

\subsubsection{BRICK FOUNDATIONS OF THE GOTHIC ST. JAMES CHURCH IN TORUŃ}

Bricks were one of the first objects to be dated using luminescence techniques, with the earliest measurements made over fifty years ago [116,117]. However, compared to pottery or geological sediment, brick dating is not often reported in the literature [118-127].

St. James Church in Toruń, see Figure 4.3, is one of the most important gothic buildings in Poland. Construction of the current church started in 1309 with its presbytery. The magnificently pro-portioned building was constructed from bricks, with plain windows and rosettes. A series of excavations were carried out over several years to investigate the possible remains of a previous church which existed on the same site before the present church was built.

Foundations around the church were studied to provide information on the design and construction of the

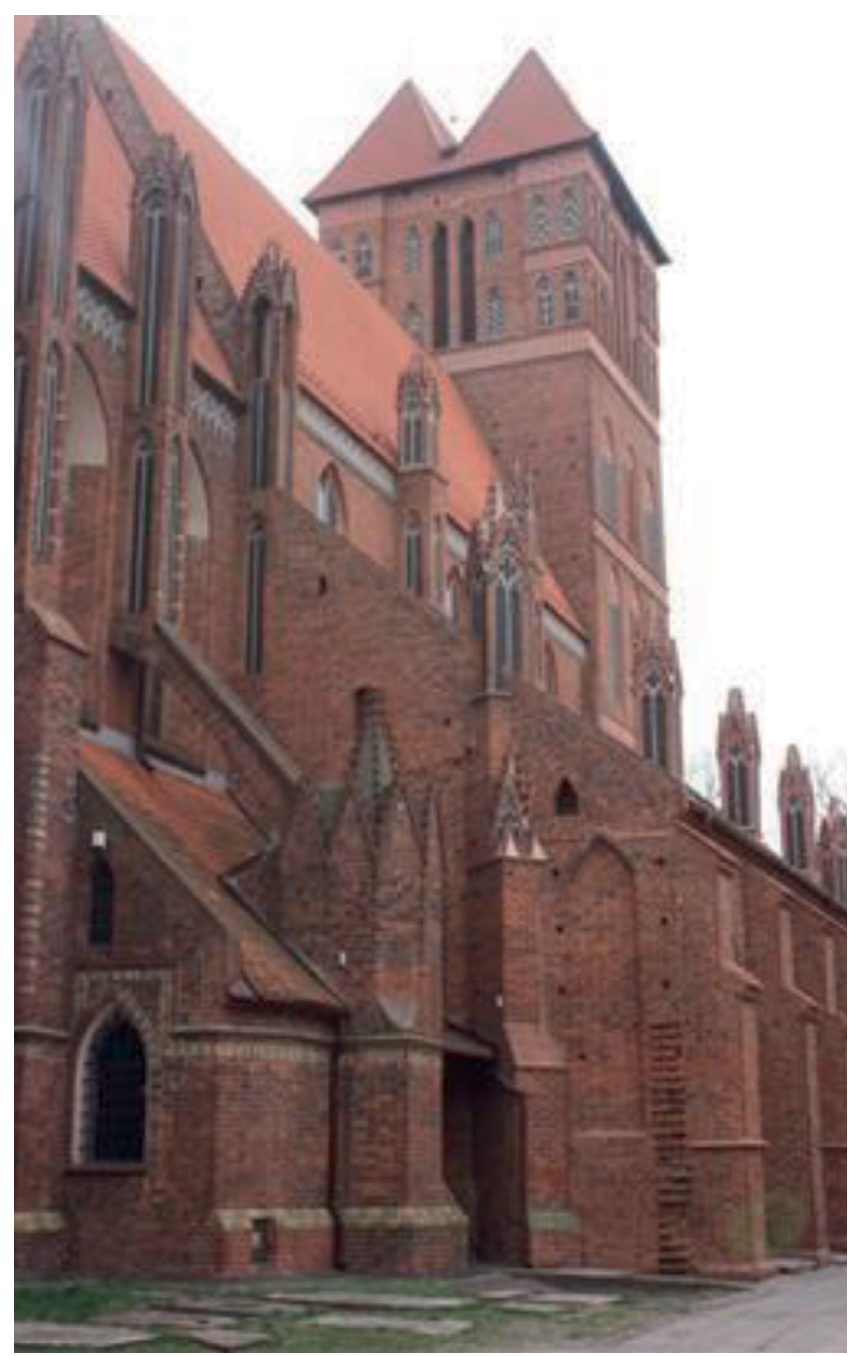

Figure 4.3: St. James Church in Torun, Poland. presbytery. In trenches on the north-western side, next to the first and second buttresses, a brick foundation with two distinct offsets was discovered. Both the buttresses resting on the foundation are at a different angle to the foundation. It is also seen that the second buttress was also considerably shorter and narrower than the foundation, and had no offsets.

There are two possible explanations for this. The first possibility is that the foundation could have supported an older brick or wooden church erected in the $13^{\text {th }} \mathrm{c}$. An alternative explanation is that it shows a change in the initial design by a builder overseeing the construction in the $14^{\text {th }} \mathrm{c}$. The excavations on the opposite, south-eastern, side of the presbytery showed much shallower and more regular foundations.

Altogether twelve brick samples were collected in five excavations carried out around the present presbytery and vestry. The luminescence dating of samples from the deep foundations on the north-western side of presbytery proved that these foundations are earlier than the wall of present basilica [123]. However, the brick samples taken from the shallower foundations turned out to be of different ages. Some had an age similar to the older bricks from the deep foundations while others had ages which coincided with the time of construction of the present gothic church. Moreover, the detailed $\gamma$-spectrometry radioactivity data revealed an analogous relationship. The bricks of similar ages had similar radioactivity characteristics.

The results of luminescence dating support the theory that a brick building existed earlier at the location of St. James Church. The parts of the building above ground were dismantled. However, its foundations were incorporated into the foundations of the new basilica and some of the bricks from the demolition were used for the construction of the new presbytery.

\subsubsection{DATING OF MEDIEVAL MANUSCRIPTS FROM THE UNIVERSITY OF SEVILLE LIBRARY}

The General Library of the University of Seville, Spain, was created in 1502 and opened to the general public in 1843. Manuscripts, books and other materials were collected from donations, inheritances and purchases made using donated funds. Its current location dates from the 1950s when the University moved to the Real Fábrica de Tabacos.

The Antique Section of the Library owns around 800 manuscripts, more than 300 incunabula, and nearly 40,000 volumes from the $16^{\text {th }}-18^{\text {th }}$ C., which form a major part of the cultural heritage of the University of Seville. They are preserved in a special security room under strictly controlled conditions of temperature and humidity. See Figure 4.4. 
In order to estimate the age of ancient manuscripts palaeographical methods are commonly applied. See Figure 4.5. These rely on the evolution of the art of writing styles and decorative patterns. They are not absolute methods, and estimates usually result in a rather wide time range. Radiocarbon dating provides an independent method which can corroborate previous findings. As AMS uses very small amounts of material, the damage caused is almost negligible.

In this study, 10 manuscripts written on parchment or paper were selected. All of these were estimated to belong to the $14^{\text {th }}$ or $15^{\text {th }} \mathrm{c}$. Samples were obtained in the Library and taken to the lab, where chemical treatments were applied to clean the material from exogenous carbon, and prepare it for AMS measurements.

In general, radiocarbon dating results agree well with palaeography estimates, but provide additional information that can help reduce uncertainty in manuscript dates. In some cases, radiocarbon dating gave dates older than previous estimates. This could arise if the parchment was reused after washing off previous writing. This was not unusual due to the high cost of parchment. Another interesting study looked at two samples from different parts of the same manuscript. The parchment looked very different and there were doubts as to whether they had been written at the same time. However radiocarbon dating confirmed that both parts were most likely contemporaneous.

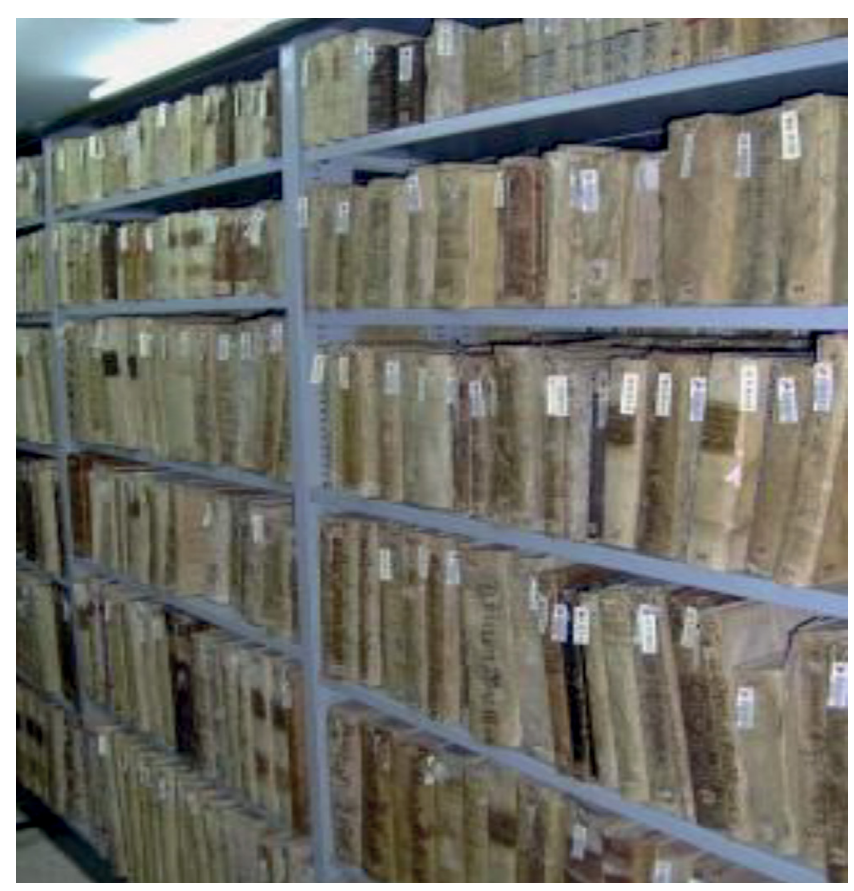

Figure 4.4: Part of the historic collection at the Library of the University of Seville.

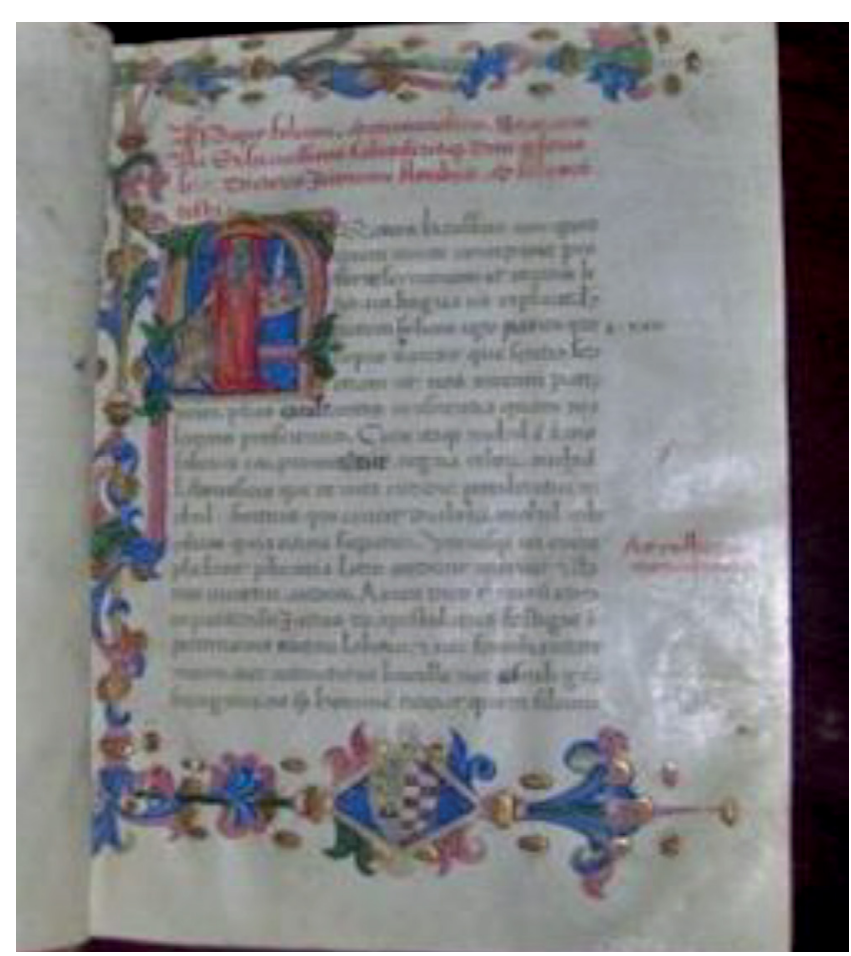

Figure 4.5: One of the manuscripts from the collection of the Library of the University of Seville investigated using AMS.

\subsubsection{THE ${ }^{14} \mathrm{C}$ BOMB PEAK ANALYSIS OF MODERN AND CONTEMPORARY ART}

The Bomb Peak is the well-known expression used to describe the huge variations of the ${ }^{14} \mathrm{C}$ concentration in the atmosphere starting from the mid-1950s as a consequence of many nuclear weapons tests. In fact, nuclear explosions produced a large excess of neutrons that induced an increase in the radiocarbon production rate. The increase was so evident that the ${ }^{14} \mathrm{C}$ concentration almost doubled in less than ten years, from 1955 until about 1963, when the Nuclear Test Ban Treaty put an end to the tests in the atmosphere.

The radiocarbon concentration then began to decrease, due to the rapid exchanges between the atmosphere and the other carbon reservoirs (i.e. the oceans and the whole biosphere). All the organisms living in that period were characterised by large variations of the ${ }^{14} \mathrm{C}$ content. The same also applies to those materials, like e.g. canvas and cardboard, used as support of artworks, which were derived from those organisms.

The radiocarbon Bomb Peak has been exploited in many fields (biology, food safety, forensics), thanks to the fact that very precise dating measurements can be performed on samples of this period. The possibility to use it for Cultural Heritage applications has been investigated too. In particular, even though we cannot precisely date contemporary artwork due to the heterogeneity of the materials and the possibility of their re-use, the Bomb Peak can be used to discover recent forgeries of artefacts that are supposed instead to have been created before 1955 , for instance in the first half of the $20^{\text {th }} \mathrm{c}$. 
A prime example of how useful the Bomb Peak can be for modern art authentication is represented by the case of the painting Contrastes de Formes of the Peggy Guggenheim collection [128].

This artwork, see Figure 4.6, supposed to be painted by the French artist Fernand Léger in 1913-14, was bought by the art collector but has never been exhibited because, already in the middle 1970s, an art critic questioned its authenticity.

A small piece of the cotton canvas was cut from the excess fabric around the frame. The sample was treated to remove any possible contamination and then reduced to graphite for the AMS measurement. The result was surprising but unquestionable: the measured radiocarbon concentration was found to be $(129.05 \pm 0.68)$ pMC (percent Modern Carbon) units. Any result larger than 100 pMC clearly indicates that the cotton plant was cut after the beginning of the Bomb Peak around 1955. As Léger died in 1955: the painting could not be original! It is a forgery, as first suspected in the 1970s.

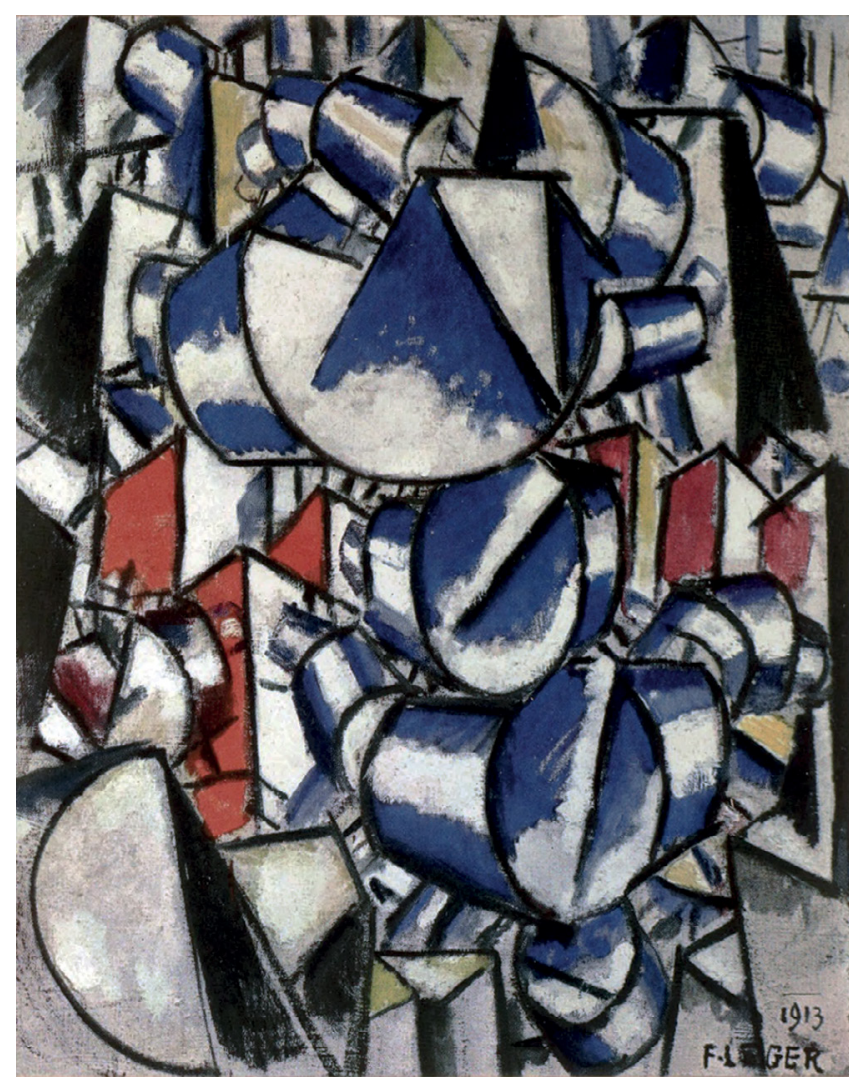

Figure 4.6: The painting Contrastes de Formes formerly attribuited to Fernand Léger, was measured with the AMS technique at the LABEC external microbeam in Florence.

\subsubsection{THE CASE OF THE ARTEMIDORUS PAPYRUS}

The so-called Artemidorus papyrus is a long papyrus scroll of very remarkable dimensions (about $2.5 \mathrm{~m}$ long and $32 \mathrm{~cm}$ high), now kept at the Museo di Antichità in Turin, Italy. It became quite famous some years ago due to a fierce debate about its authenticity. The scroll was reconstructed by assembling together many fragments (more than a hundred) found in a konvolut, i.e. a mass consisting of paper, glue and gypsum that had been probably used as the filling of a small Egyptian mummy, maybe a small animal.

${ }^{14} \mathrm{C}$ can provide a direct dating of the material constituting the manuscript support, the papyrus; indirect information can be also inferred using analytical techniques such as Ion Beam Analysis (IBA) to measure the composition of the ink. In fact, different inks have been used in different historical periods; for example, iron gall inks were used routinely only since the late Middle Ages, while carbon black inks were commonly used from a few millennia BC.

When papyrologists reconstructed the document, they found a text written in ancient Greek reporting the beginning of the second book of Geographoumena, a treatise on geography by Artemidorus of Ephesus (about $1 \mathrm{c}$. BC).

The text describes the Iberian Peninsula and it is combined with a sketch of a map. Moreover, the rest of the surface is full of drawings: on the recto there are sketches of heads, hands and feet, while on the verso there are images of real (e.g. a tiger, a giraffe and some fish) and fantastic (e.g. a griffin) animals. While some scholars think that it is the first known transcription of the work of Artemidorus, later used as a sort of sketch book before being thrown away to become part of a mummy, some others claim it is a $19^{\text {th }} \mathrm{c}$. forgery, probably made by the Greek forger Costantinos Simonides. In this context, the issue of dating is fundamental.

For ${ }^{14} \mathrm{C}$ dating, five samples were collected from five different areas of the scroll, see figure 4.7, in order to avoid possible ambiguities due to the fact that the document consists of several different fragments. The mass of each sample was less than $5 \mathrm{mg}$. Samples were pre-treated and converted to graphite to be measured by AMS.

Figure 4.7: Recto of the Artemidorus papyrus: the locations of five samples collected for radiocarbon dating are indicated.
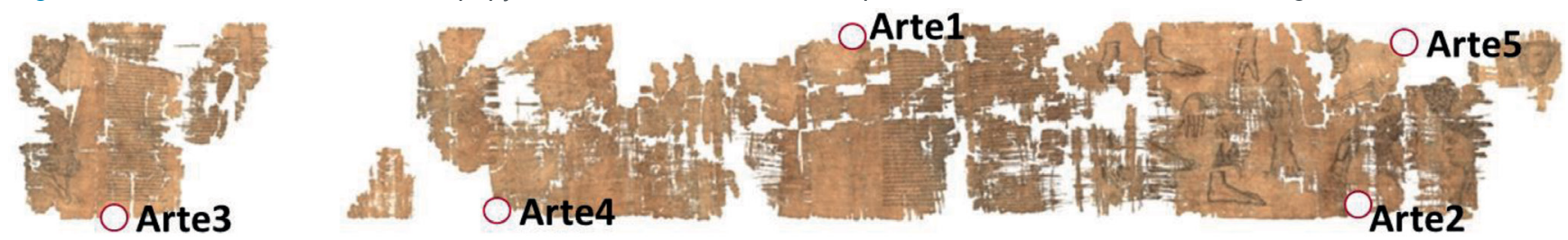


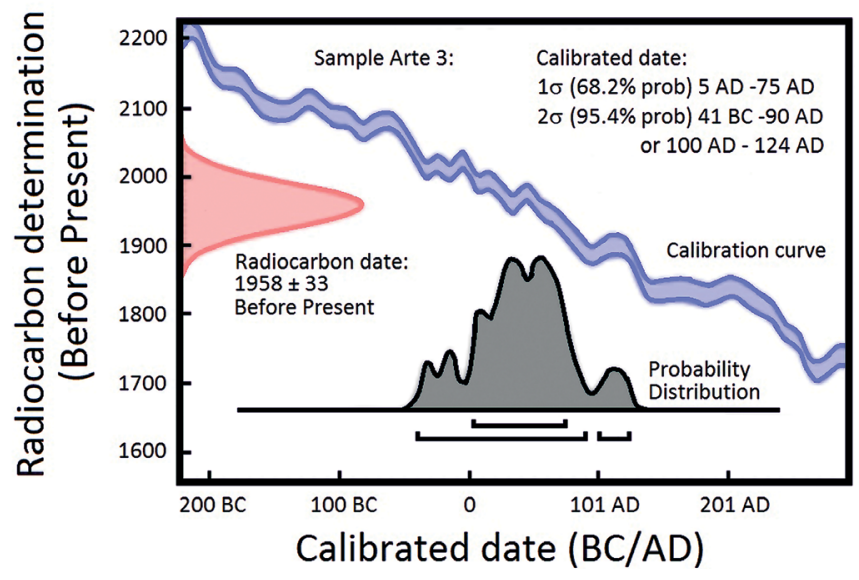

Figure 4.8: Radiocarbon measurement of the sample Arte 3 from the Artemidorus papyrus [131]. The conventional radiocarbon age (red) was found to be $1958 \pm 33$ years before the present time. Using the $0 \times \mathrm{Cal} v 4$ and IntCal0 4 calibration curve (blue) the calibrated date is within the range $41 \mathrm{BC}$ to $90 \mathrm{AD}$ or $100 \mathrm{AD}$ to $124 \mathrm{AD}$, with $95 \%$ probability.

AMS measurements were performed at INFN-LABEC, Florence (samples Arte1, Arte2, Arte3) and at CIRCE, Second University of Naples, Caserta (Arte 4 and Arte5). All the results were in good agreement with each other and consistent with the hypothesis that the papyrus support is an original material of the period between the $1^{\text {st }} \mathrm{c}$. BC and the $1^{\text {st }} \mathrm{c}$. AD.
The results of radiocarbon measurements on sample Arte 3 are shown in figure 4.8.

Ink composition was studied by PIXE (Particle Induced $X$-ray Emission) and BS (Backscattering Spectroscopy); all the measurements were done at INFN-LABEC, Florence, at the external microbeam set-up, using a $2 \mathrm{MeV}$ proton beam as the probe $[129,130]$.

Elemental mapping by PIXE showed no evidence of elements that may be associated with iron gall inks: indeed, carbon-based inks are organic and thus invisible to PIXE measurements in external environments. On the contrary, BS spectra showed an enrichment of carbon and oxygen corresponding to the ink trace. This suggested that the ink used was organic and thus compatible with materials typically used in ancient Greek and Roman times [129-131].

We can thus conclude that ${ }^{14} \mathrm{C}$-AMS and IBA-PIXE measurements have contributed new information supporting the hypothesis of originality of the materials used in the papyrus. However, such a difficult authenticity issue cannot be solved without the fundamental expertise of historians, phylologists and papyrologists.

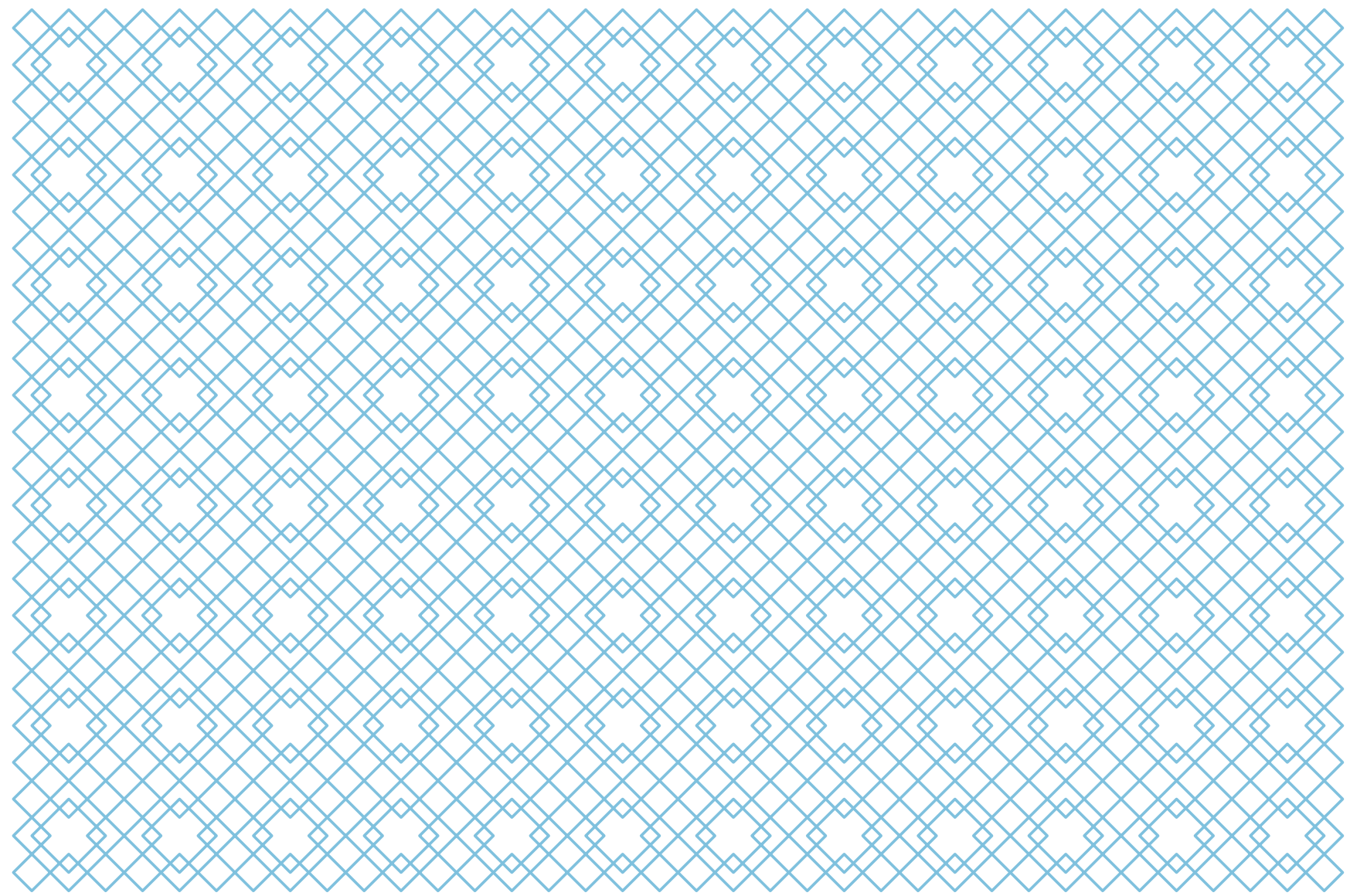




\section{COMPLEMENTARY METHODS: $\mathrm{Y}$-BEAM TECHNIQUES, X-RAY FLUORESCENCE (XRF) AND NUCLEAR MAGNETIC RESONANCE (NMR)}

In this chapter we look at a range of techniques which can provide complementary information on the properties of Cultural Heritage objects. The first of these, Nuclear Resonance Fluorescence (NRF), is a novel technique which tunes high energy $\mathrm{Y}$-rays to particular nuclei. The high energy $Y$-rays can probe the volume of cultural heritage artefacts, providing elemental analysis information. The technique of Computed Tomography (CT), using high energy $y$-beams, provides a new imaging method to map the internal densities of artefacts. These techniques complement the more established method of X-ray Fluorescence (XRF), which identifies elements, near the surface of objects, on the basis of their characteristic $X$-ray emission. Nuclear Magnetic Resonance (NMR) imaging is another imaging technique, extensively used in medical imaging. However, its use in imaging Cultural Heritage artefacts is less well known. Here the use of NMR probes to study the restoration of painitings is described.

\subsection{BASIC PRINCIPLES}

\subsubsection{Y-BEAM ANALYTICAL METHODS}

In many cases it is desirable to study the bulk composition of Cultural Heritage objects. The ideal probe for in-depth element analysis should provide a non-destructive and non-invasive study and should not influence the object in any way. It should have a high penetrability and should allow 2D or 3D imaging of the object. It should be sensitive to small element admixtures in the object matrix for studies of its provenance, i.e. the origin of the raw material, the workshop where it was produced, or the technique used for its production.

Atomic and nuclear IBA techniques can only be applied for surface studies of objects. This limitation is due to the finite range of the ion beam in matter, which is typically a few tens of microns. Neutron-activation analytical techniques meet the requirements for indepth studies to some extent. However, they depend on neutron-capture cross-sections, which are strongly isotope dependent, and generate a problem of residual radioactivity. A probe, which meets fully the requirements for in-depth analysis is a brilliant quasi-monochromatic gamma beam with energies from a few hundred keV to several $\mathrm{MeV}$, remaining well below the photonuclear reaction energy threshold.

The Extreme Light Infrastructure - Nuclear Physics (ELI-NP) is a pan-European laboratory currently under construction at Magurele, Romania. It will provide $y$-beams with high brilliance and narrow bandwidth, orders of magnitude better than existing facilities.

Such an ultra-bright, energy-adjustable and monochromatic $\gamma$-ray beam in the range $0.2-19.5 \mathrm{MeV}$ produced by the laser-Compton backscattering technique is ideal for non-destructive testing applications. Consequently, this $Y$-beam satisfies the criteria for investigations of largesized objects, with added capabilities of isotope detection and $3 \mathrm{D}$ imaging through the use of the nuclear resonance fluorescence (NRF) technique.

This advantage opens up numerous potential applications of NRF methods for characterising the internal structure of valuable historical artefacts, industrial products, etc. In contrast to other nuclear methods used 
for composition analysis, NRF requires very limited manipulation of the materials and does not noticeably affect the investigated objects.

\section{NUCLEAR RESONANCE FLUORESCENCE}

The experimental program of the ELI-NP laboratory includes the development of different applications, using NRF. NRF is an attractive non-destructive analysis method because it provides signatures for a wide variety of materials, signatures that can be used to characterise the irradiated samples. In general, the $y$-beam is tuned to the energy of a specific state of the isotope of interest and the absorption of $y$-rays is monitored. Thus, the method provides selectivity for all elements.

So far, this technique has not yet been applied to studies of archaeological artefacts or work-of-art objects. To demonstrate the sensitivity of the NRF method the concentration of ${ }^{13} \mathrm{C}$ in a diamond sample has been studied [132], confirming the results of Raman scattering while demonstrating the feasibility of identifying milligram quantities from the target nuclide.

Two major applications of $y$-beams which are appropriate for Cultural Heritage studies are envisaged at ELI-NP: measurements based on the NRF technique, and the radioscopy and tomography of large objects. Both exploit the unique characteristics of the ELI-NP $\mathrm{Y}$ - beam. The non-destructive assay based on high-brightness $y$-rays can be successfully applied to elemental analysis of largesized objects. Radioscopy and computed tomography performed at ELI-NP have the potential to achieve high spatial resolution and high contrast sensitivity. Practically, using these $y$-beams it is possible to detect and measure nuclides non-destructively.

This is a key technology for nuclear applications such as trace element analysis of Cultural Heritage objects. Moreover, the new source of $\gamma$-rays could be a perfect

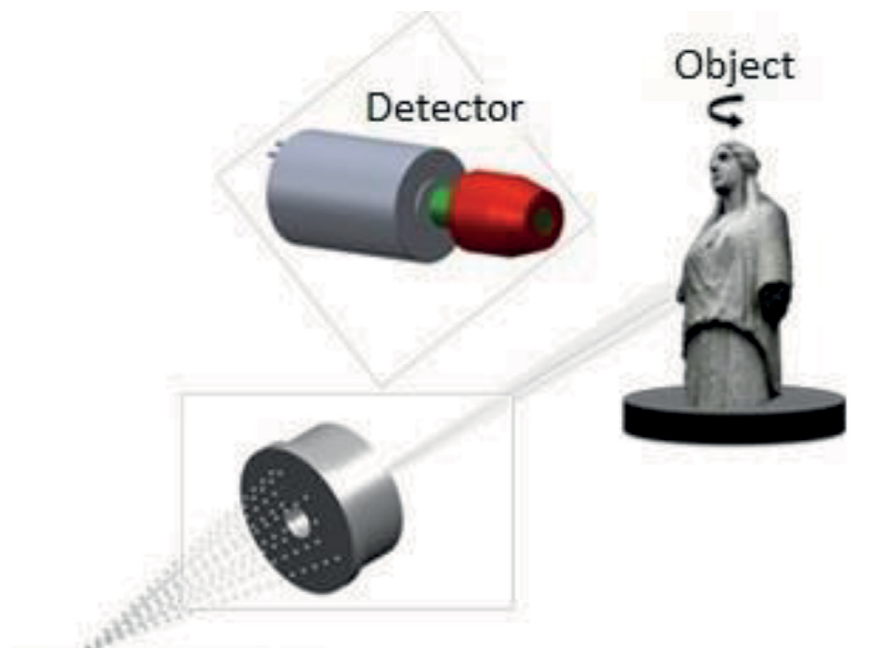

\section{Gamma source}

Figure 5.1: Schematic view of the backscattering NRF setup. solution to meet all the technical requirements for investigations of large-sized and complex archaeological artefacts and works of art.

A few studies have been carried out in other fields of science which demonstrate the high potential of the NRF method. Its applicability was demonstrated for chemical compounds and organic materials by non-destructive inspection of a shielded explosive, where the abundance ratio of chemical components, ${ }^{12} \mathrm{C} /{ }^{14} \mathrm{~N}$, of a melamine compound was successfully extracted [133].

The sensitivity of the method to the presence of heavy metals in foods or other organic matrices is in the range from $0.1 \mathrm{mg} / \mathrm{kg}$ up to $200 \mathrm{mg} / \mathrm{kg}$. As noted above, the applicability of the NRF method is quite general and broad. Moreover, the NRF method can be used in conjunction with tomography to produce elemental maps in objects of various compositions. For example, a 2D-image of the isotope distribution of ${ }^{208} \mathrm{~Pb}$ was demonstrated recently by using the NRF technique [134].

The two detection schemes used for NRF-based investigations are the scattering and transmission methods. In both cases the $y$-beam is used to induce the resonant absorption while the de-excitation photons are detected either in a backscattering geometry or a transmission, self-absorption, geometry. The general setup employed in the scattering NRF experiments is illustrated in Figure 5.1. Here the investigated object is placed in the beam and the resonant photons are detected in a backscattered geometry by a detector located away from the beam. Except for the resonant photons, all the other scattered photons have a low energy due to the nature of Compton scattering. Therefore the high energy resonant photons can be easily distinguished.

The second detection method proposed for NRF measurements is the self-absorption or the transmission method (see Figure 5.2). In this method the $y$-beam, which has penetrated the studied object, strikes a sample (witness foil) that contains an isotope of interest. This isotope is expected to exist in the object with a small concentration. The detection of resonant photons scattered by the witness foil is carried out with an offbeam detector in a backscattering geometry, often referred to as a notch detector. The flux of the transmitted off-resonance photons may be detected using an in-beam detector placed downstream of the witness foil as pictured in figure 5.2. Collimators may be used to define the beam and to remove scattered radiation.

Contrary to the scattering NRF detection method where the photons that undergo NRF in the object are registered in the detector, in the transmission method the notch detector measures the attenuation of the resonant photons in the witness foil. The measured attenuation of particular resonant photons in the witness foil can indicate the amount of the isotope of interest in the investigated object. 


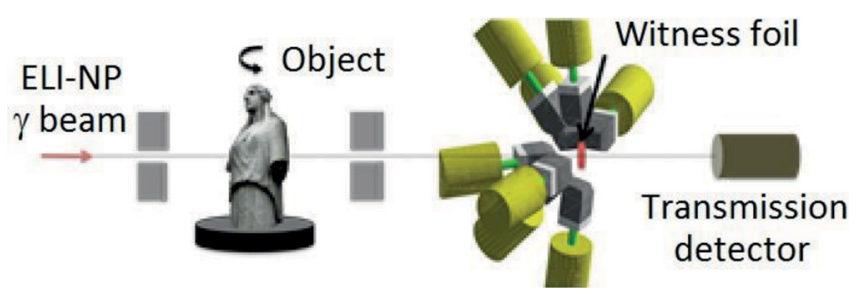

Figure 5.2: Schematic view of the trans-mission NRF setup.

While the application of the NRF method in different fields (e.g. safeguards and nuclear waste management as well as Cultural Heritage studies) shows great promise, there are some technical issues that need to be taken into account when choosing the test case for the initial feasibility estimate. The point is that information about the NRF resonances is not known for many nuclides of interest and an extensive database still needs to be developed.

An estimate of the experimental opportunities for such measurements at the ELI-NP gamma-beam system was made through comparison with a recent NRF study [135] performed at the High-Intensity Gamma-Ray Source (HlyS) at Duke University, USA, the world's present leading $y$-beam facility [136]. In a transmission experiment, the $y$-beam was fired at a $1.3 \mathrm{~cm}$ thick slab of depleted uranium (DU), shielded by a $1.3 \mathrm{~cm}$ slab of tungsten. A thick DU target was used as a witness foil, followed by a Cu foil for beam normalisation. Four high purity germanium (HPGe) coaxial detectors were positioned at backward angles relative to the beam, facing the witness foil. A total of $110 \mathrm{NRF}$ DU events were recorded in about 25 hours in the most unfavourable case [135].

The conclusion was reached that a six-hour measurement would be required for a six-sigma level detection of a sensitive material in the object when using the 100 photons/(eV s) flux at Hlys [135]. At ELI-NP, using a conservative value for the spectral density of $2.8 \times 10^{4}$ photons / (eV s) [137], the same statistics could be reached in less than two minutes.

\section{ISOTOPE IMAGING}

A high penetrability and a weak elemental dependence of the attenuation co-efficients of several MeV $\mathrm{Y}$-rays make it possible to visualise the density distribution inside a massive object by the technique of computed tomography (CT) [138]. This measures the transmission of $Y$-rays through objects. In addition, the distribution of isotopes of interest inside an object could also be visualised by measuring NRF $y$-rays [139]. The attenuation of NRF $y$-rays, absorbed in the investigated object, can be inferred from the detection of the NRF $Y$-ray scattered by a notch target (witness foil) placed downstream.

To examine the feasibility of CT imaging of specific isotopes in high dense objects at ELI-NP, a simulation study was performed [139] using the design values of the laser Compton backscattered $y$-ray beam [137]. A simulation setup based on the NRF transmission method was used to obtain the transmission factor of the $y$-rays of $2176 \mathrm{keV}$ (excitation energy of ${ }^{238} \mathrm{U}$ ) through an object that contains both ${ }^{235} \mathrm{U}$ and ${ }^{238} \mathrm{U}$. A clear distinction between the ${ }^{235} \mathrm{U}$ and ${ }^{238} \mathrm{U}$ distributions in the investigated object can be made when the transmission factor of on-resonance $y$-rays normalized by that of off-resonance $y$-rays is used for the CT image reconstruction. This example illustrates that the proposed method to visualise the isotope distribution is feasible at ELI-NP.

In short, so far the NRF technique has not yet been applied for Cultural Heritage studies. However, in view of its universal applicability, we expect that in the near future the international Cultural Heritage community will have at its disposal a new powerful tool for precise element analysis and 3D imaging of bulk objects. This technique will be developed and implemented at ELI-NP, taking advantage of the brilliant, quasi-monochromatic, tunable $\mathrm{Y}$-beams which will be soon available in the laboratory.

\subsubsection{X-RAY FLUORESCENCE (XRF) SPECTROSCOPY}

$\mathrm{X}$-ray Fluorescence (XRF) spectroscopy is often used nowadays in the analysis of Cultural Heritage objects because it is a non-invasive, non-destructive, highly sensitive and quantitative multi-elemental technique that in many cases can be used directly on the surface of objects to be studied without any prior sample preparation.

When a low energy $\mathrm{Y}$-ray or $\mathrm{X}$-ray beam impinges on a sample it is very probable that a photoelectric effect takes place and an electron from one of the internal atomic shells will be ejected. The photon from the impinging beam will disappear and all its energy will be transferred to the ejected electron which acquires a kinetic energy equal to the difference between the energy of the incident photon and its binding energy in the atom. The creation of a vacancy in the internal atomic shells leaves the atoms in an excited state and, in order to return to the ground state, an electron from a higher level will fill the vacancy. The difference in energy between both electronic levels will be immediately radiated in the form of X-rays or, alternatively, Auger electrons.

As the electronic levels are characteristic of the different elements of the periodic table, the emitted X-rays (or Auger electrons) are also characteristic. The emission of characteristic X-rays can be used as a fingerprint of the different chemical elements that a sample is composed of. The process of excitation by a primary X-ray beam and re-emission of characteristic X-rays is known as X-ray fluorescence (XRF) and can be used as an analytical technique for elemental analysis [140]. 
However, it is necessary to take some precautions when analysing Cultural Heritage objects due to the relatively low depth penetration into the sample, which could be a problem if the object surface suffers any alteration from its bulk composition. Indeed, the limited penetration of the primary beam (only a few tens or hundreds of micrometres, depending of its energy and the sample composition) and also the attenuation that the characteristic $X$-rays undergo from the sample to the detector, restrict the accessible depth which can be analysed.

XRF has been applied to almost every kind of Cultural Heritage object [140,141]: paintings, glasses, jewels, coins of different alloys, gems, marbles, bones, ceramics, inks, graphic documents, enamel, porcelain, obsidian, ivory, etc. . In all these materials, the non-destructive, multi-elemental and quantitative character of the technique and its high sensitivity has provided very useful information for studies of provenance, authentication, etc.

\subsubsection{NUCLEAR MAGNETIC RESONANCE}

The Nuclear Magnetic Resonance (NMR) technique employs strong magnetic fields [142]. Its application in medical diagnostics is known as Magnetic Resonance Imaging (MRI). In chemistry NMR spectroscopy is used to analyse the structure of molecules. NMR spectroscopy makes use of homogeneous magnetic fields while inhomogeneous fields are used in MRI and in NMR relaxation studies of solid materials, which provide information on their physical properties [143].

Nuclear Magnetic Resonance (NMR) is a physical resonance phenomenon employing magnetic fields. It is best known from its use in medical diagnostics where it is referred to as Magnetic Resonance Imaging (MRI). NMR spectroscopy is another important branch of NMR employed in chemistry to analyse the molecular structure of chemicals. NMR spectroscopy requires extremely homogeneous magnetic fields.

The measurement of NMR relaxation is popularly known as NMR relaxometry [144,145]. Relaxometry can be performed in the inhomogeneous stray field outside a magnet with spatial resolution reminiscent of MRI studies inside the magnet. Whereas homogeneous magnetic fields are typically generated in dedicated laboratories by superconducting magnets producing high fields, inhomogeneous magnetic fields are readily produced with compact permanent magnets, which can be transported to the site of a measurement [146-148]. Also note that recently compact benchtop NMR instruments have become available for chemical analysis [148].
All atomic nuclei which have an odd number of protons, an odd number of neutrons or an odd number of both, are magnetic and give an NMR signal. All magnetic nuclei have a quantum mechanical property known as spin and are often just referred to as spins. In the presence of a static magnetic field spins precess with a characteristic frequency around that field. See Figure 5.3.

Resonance in NMR is commonly tested by invoking an impulse response from nuclear magnetization in a magnetic field with at least one radio-frequency (rf) pulse. There are many different $r f$ pulse sequences which may be used to investigate different aspects of condensed matter. NMR studies of materials exposed to the inhomogeneous stray field of magnets are not limited to small samples and can be conducted on complete objects and even large walls $[149,150]$.

Using stray-field NMR it is not possible to measure spectra for chemical analysis but it is possible to noninvasively determine elemental density, diffusion, and NMR relaxation. These quantities provide information that can be interpreted in terms of material elasticity, moisture content and moisture transport. Applications of mobile stray-field NMR analyses range from testing roads and bridges to analysing master paintings and sealed food items. Just about any object containing hydrogen atoms can be studied, because ${ }^{1} \mathrm{H}$ is the most sensitive NMR-active nucleus.

\subsection{INSTRUMENTATION OF COMPLEMENTARY METHODS}

\section{XRF INSTRUMENTATION}

The instrumentation needed for XRF is usually not too complex and essentially has two main components: the $\mathrm{X}$-ray generator for the primary beam and a detector to collect the secondary characteristic $X$-rays emitted by the sample to be analysed. Usually, radioactive sources or $\mathrm{X}$-ray tubes are used as generators for the primary beam.

Sometimes, a more intensive X-ray source is needed and Synchrotron radiation is used as primary beam (SRXRF or SRIXE). For detection, two kinds of systems can be used: energy dispersive (EDS) or wave-length dispersive (WDS) detectors. With EDS detectors, usually semiconductor devices, a wide range of energies can be simultaneously recorded and a multi-elemental analysis of the sample can be directly obtained.

In WDS systems, diffraction crystals are used and typically only one wavelength is counted at a time. Using WDS detectors, a sequence of successive analyses are needed to complete the multi-elemental analysis of an object although better energy resolution, compared with 
EDS detectors, can be achieved. Consequently interference between $\mathrm{X}$-rays from elements with emissions close in energy is more easily avoided.

EDS systems have been miniaturised in the last few years, so there are many portable commercial systems that allow in situ measurements. More recently, such systems have become increasingly used in the analysis of Cultural Heritage objects, and offer the possibility of performing a quantitative analysis of an object with good accuracy in only a few minutes [151].

Another important development in recent years has been the use of polycapillary fibres in micro-focus $X$-ray tubes to produce micro $X$-ray beams with a lateral resolution of a few tens of micrometres. When using these micro-XRF systems with detectors which are also equipped with a polycapillary lens, a confocal XRF analysis of the sample can be carried out, allowing detailed information to be obtained non-destructively.

\section{NMR INSTRUMENTATION}

The precessional angular frequency $\omega$ is called the Larmor frequency. It is proportional to the strength $B_{0}$ of the magnetic field, $\omega=\gamma B_{0}$.

Typically $\omega$ is in the radio-frequency ( $\mathrm{rf}$ ) regime. Here the constant $y$ is the gyromagnetic ratio which is a property of the type of nucleus. The precessing nuclei act like miniature magnets and tend to align with the magnetic field in competition with thermal energy, which breaks the alignment through random motion. As a result only about 10 in a million spins are aligned on average with the magnetic field at room temperature, giving rise to a rather small NMR signal. The average ratio $n_{\text {down }} / n_{\text {up }}$ of nuclei anti-parallel or parallel to the magnetic field is estimated from Boltzmann statistics,

$$
n_{\text {down }} / n_{\text {up }}=\exp \left(-\Delta E / k_{B} T\right),
$$

where the energy difference $\Delta E=h \omega /(2 \pi)$ between the two alignment states scales with the Larmor frequency $\omega . h$ is Planck's constant, $k_{B}$ is Boltzmann's constant and $T$ is the absolute temperature in Kelvin.

As soon as the nuclei experience the magnetic field they start to align while precessing and build up a magnetic polarisation or longitudinal magnetisation $M_{z}(t)$ parallel to the magnetic field $B_{0}$, which by convention is taken to be in the z-direction. Usually this process is exponential with a time constant $T_{1}$ known as the longitudinal relaxation time,

$$
M(t)=M_{0}\left(1-\exp \left\{-t / T_{1}\right\}\right) .
$$

The longitudinal magnetisation (Figure 5.3a) can be rotated away from the longitudinal direction $z$ with a magnetic field $B_{1}$ oscillating in resonance with the precessing spins and oriented orthogonally to the main magnetic field $B_{0}$. If the $B_{1}$ field is turned off after a quarter rotation, the longitudinal magnetisation ends up in the $x y$ plane and becomes transverse magnetisation. Following this rf pulse, the transverse magnetisation precesses around the $B_{0}$ field with the Larmor frequency $\omega$. The precessing magnetisation acts like a rotating magnet, which induces a voltage in a nearby receiver coil, which is recorded and further processed. As the precession continues individual magnetisation components run out of phase and the magnetisation spreads out in the $x y$ - plane.

The time related to this loss of coherence in the $x y$-plane is called the transverse relaxation time. It is denoted by $T_{2}$ if the coherence loss arises in a homogeneous magnetic field and by $T_{2}$ * if it happens in an inhomogeneous field. In simple cases the transverse relaxation of the recorded signal $s$ decreases exponentially with time $t$.

$$
s(t)=s_{0}\left(1-\exp \left\{-t / T_{2}\right\}\right)
$$

The signal decay in a homogeneous field arises from time-dependent local magnetic field fluctuations, due to molecular motion, and accompanying destructive interference of magnetisation components. In an inhomogeneous field the magnetisation components experience locally different but time-invariant magnetic fields. The latter signal attenuation can be eliminated using echoes, which stroboscopically refocus the transverse magnetisation components.

In an inhomogeneous field the magnetisation components experience locally different but time-invariant magnetic fields. The latter signal attenuation can be eliminated using echoes, which stroboscopically refocus the transverse magnetisation components.

The NMR-MOUSE (Nuclear Magnetic Resonance - MObile Universal Surface Explorer) is a singlesided NMR sensor (Figure 5.3b), with permanent magnets and a surface rf coil, which is used to perform experiments and record data.

This process can be repeated many times and is done so in the popular Carr-Purcell-Meiboom-Gill (CPMG) sequence (Figure 5.3c). Important times in the CPMG sequence are marked on the time line in Figure 5.3e.

At $t_{\mathrm{a}}$ the $90^{\circ}$ pulse rotates the magnetisation into the tansverse plane. At $t_{b}$ the rf pulse is turned off and the transverse magnetisation starts to decay with time constant $T_{2}{ }^{*}$ in the inhomogeneous magnetic field. The decay lasts until time $t_{c}$. At $t_{d}$ a $180^{\circ}$ pulse flips the magnetisation components to the opposite side in the transverse plane so that the slower components are now ahead of the faster components.

At time $t_{f}$ the fast components have caught up with the slower ones and an echo forms. From then on the procedure repeats, and an echo train is generated (Figure 5.3d) [148]. The echoes are recorded and the envelope of the echo train is analysed to obtain the transverse relaxation time $T_{2}$ and the spin density. 


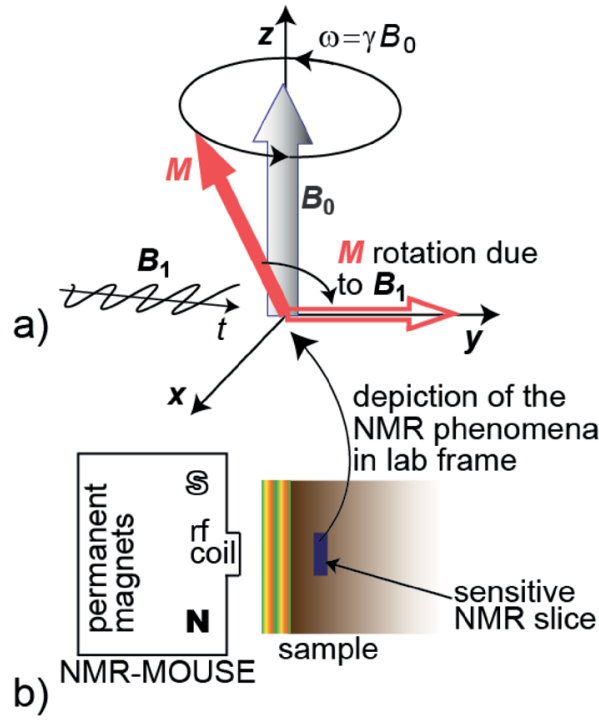

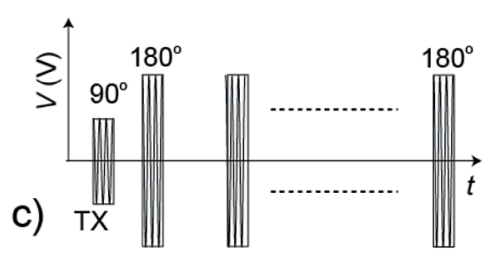

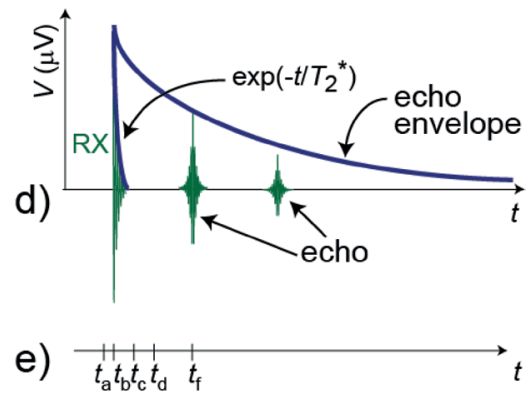

Figure 5.3: NMR relaxometry and the NMR-MOUSE. a) Illustration of the magnetisation vector $\boldsymbol{M}$ interacting with a magnetic field $\boldsymbol{B}_{1}$ in the rotating frame which oscillates with precession frequency $\omega$. M rotates about both fields, $\boldsymbol{B}_{1}$ and $\boldsymbol{B}_{0}$. b) Diagram of an NMR-MOUSE next to a sample with the sensitive slice inside. c) The rf pulses from the transmitter TX rotate the magnetisation through $90^{\circ}$ and $180^{\circ}$. d) Magnetisation echoes recorded as a voltage signal by the receiver $\mathrm{RX}$. The decay time of the envelope determines the transverse relaxation time $T_{2}$. e) Time line.
For non-destructive testing, the magnetic field is provided by the strong field of an array of permanent magnets. Two permanent magnets are placed in an anti-parallel orientation on a yoke to form a U-shaped combination. The geometry is carefully chosen to create a thin flat region with a particular magnetic field a few millimetres away from the surface of the magnet combination. A strong magnetic field gradient pointing towards the surface keeps the region of constant magnetic field thin.

A surface coil is placed between the magnets and the flat field region. This defines the sensitive slice from which the NMR signal is collected (Figure 5.3b). This slice is generally between $1 \mathrm{~mm}$ and $25 \mathrm{~mm}$ from the coil, depending on the model of the NMR-MOUSE. Relaxation experiments are performed with the sensitive slice at a particular depth.

NMR measurements are then generally repeated with the sensitive slice at several depths within the sample and the recorded data are used to create a depth profile. In this process the sensor is automatically moved away from the sample using a precision stage operated under computer control.

\subsection{APPLICATIONS OF COMPLEMENTARY METHODS}

\subsubsection{A TRIPTYCH BY MARTIN DE VOS}

Martin de Vos was one of the most important Flemish painters. He was active in Antwerp in the second half of the $16^{\text {th }} \mathrm{c} .(1532-1603)$ and had great influence on later artistic periods. Many altarpieces in Antwerp churches were created by him and his workshop. Several of those can be found today in different museums around the world. The Fine Arts Museum in Seville conserves one of his works, a triptych of high artistic quality from the St.
Augustine Monastery in Seville, which was painted around 1570 (see Figure 5.4). In the past several restorative interventions were carried out on the altarpiece, however not much is known about the pigments and materials applied, which led to the present research.

All three panels were studied with non-destructive techniques that allow in situ analysis and do not require extraction of samples. The paintings were examined with UV light to observe later interventions. This is possible due to the visible UV fluorescence obtained by radiation of ultraviolet (UV) rays from the lamp that reacts with the material. Next, infrared reflectography (IRR) was applied in order to discover possible preparatory drawings and pentimenti, that is, changes in the composition of figures.

The principal objective was to obtain data on the pigments, painting supports, later interventions and possible preparatory drawings hidden beneath the colour layers. As the wavelengths of IR rays are higher than that of visible light, they emerge from deeper in the surface and allow us to see what is hidden under the painted layers. A special IR camera, placed on a semi-automatic support, with two halogen light reflectors, was used for analysis (Figure 5.5).

As a further step, X-ray Fluorescence (XRF) was used for material analysis. Portable equipment, comprising an X-ray tube and a silicon drift detector (SDD), was used (See Figure 5.6).

Two lasers, coupled to the tube, determine a fixed distance to the irradiated point and therefore provide a direct comparison of spectra obtained from different analysed points. 

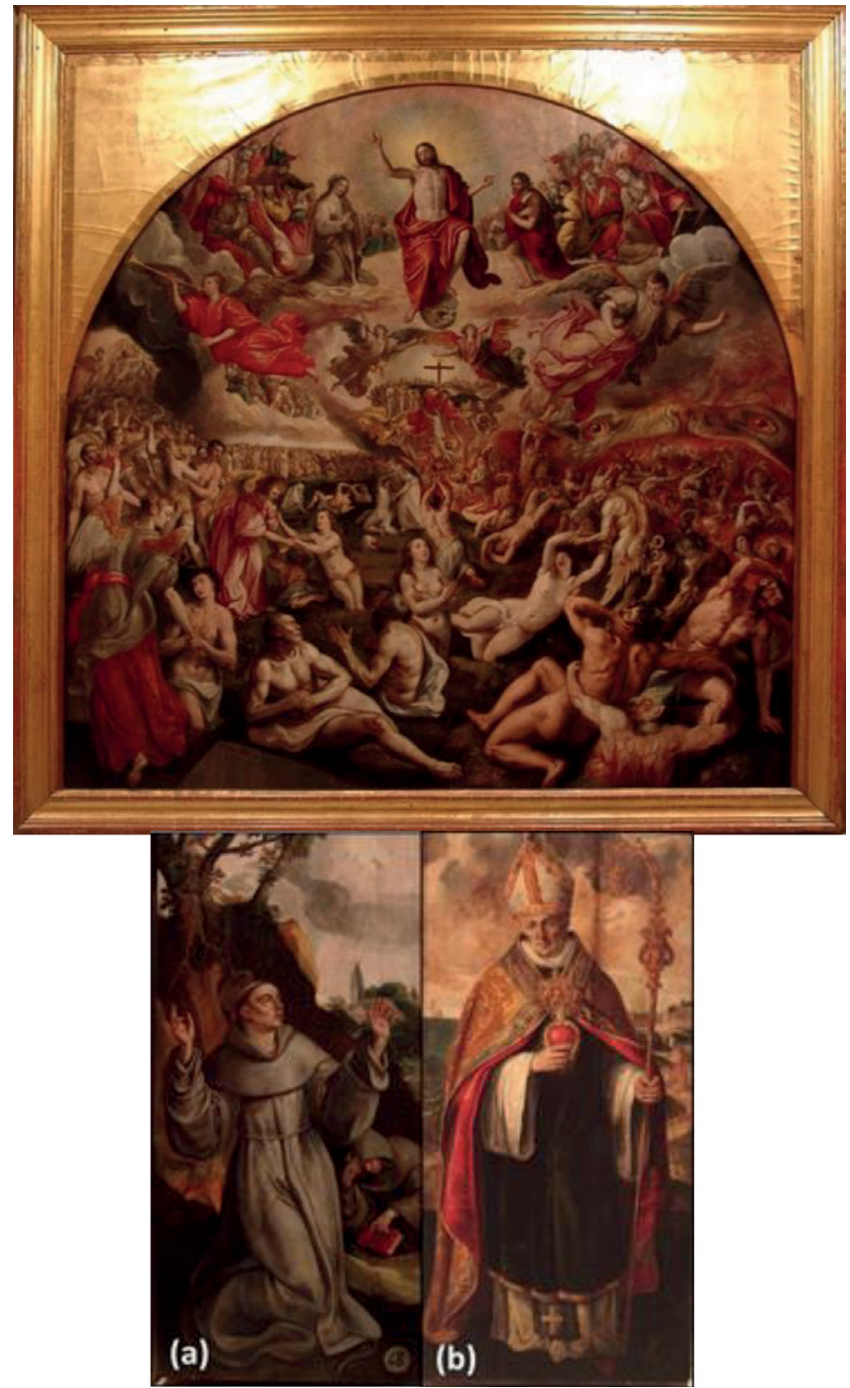

Figure 5.4: Top: Last Judgement, the large central panel of the triptych from St. Augustine Monastery in Seville (Spain). Bottom: Two small side panels from the same triptych: (left) St. Francis of Assisi, (right) St. Augustine. All three panels were painted by Martin de Vos around 1570. Fine Arts Museum, Seville.

The XRF technique enables an elemental analysis and can identify chemical elements with Z>14 (Si). This allows inorganic pigments to be identified. On every painting, several points were selected that include different colours, tonalities, shadows, highlights, sampling both the original surface and later interventions. Pigments are identified through a semi-quantitative analysis of characteristic X-ray energies, which correspond to specific elements.

Under UV light wide brownish areas are observed, revealing later interventions. The lower part of the central panel was almost entirely restored, as were the joints between the wooden panels. Also large areas of both of the side panels have been retouched (Figure 5.7).

IR reflectography was applied with the principal objective of discovering preparatory drawings by Martin de Vos. These are usually carried out by the principal artist himself and reveal his dexterity; the preparatory drawing

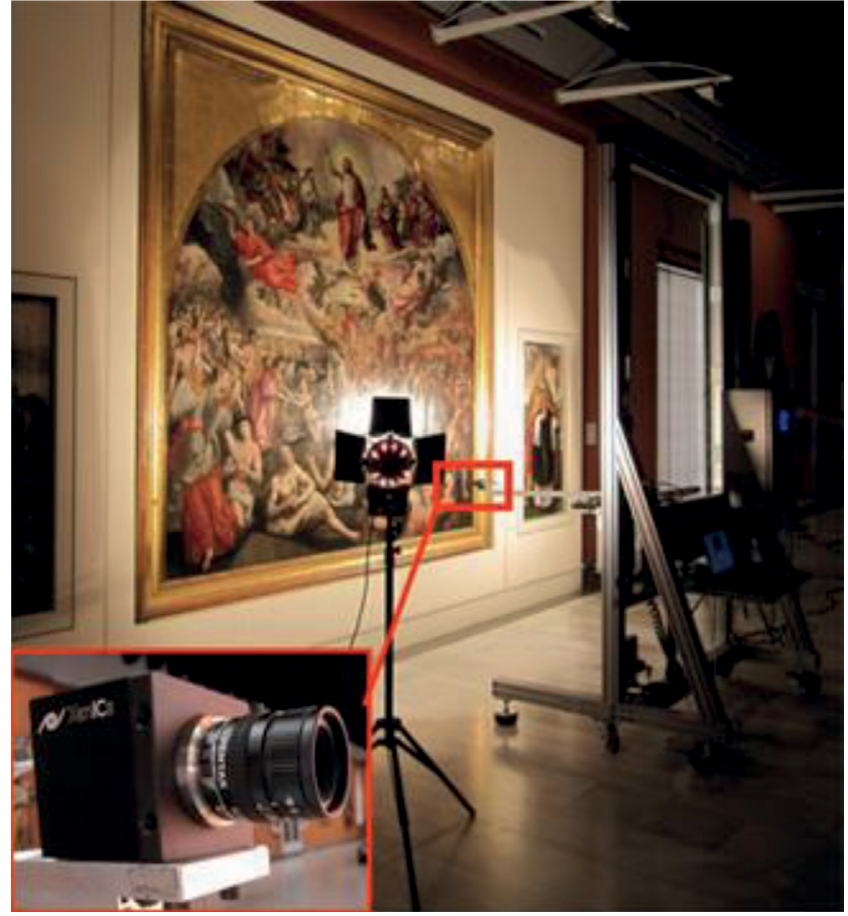

Figure 5.5: The triptych analysed in situ by IRR, placed on a semi-automatic support. The inserted image shows the InGaAs camera.

could be just a sketch or could be much more detailed. It can also reveal changes made to the composition, as one found in the central panel of the triptych (Figure 5.8). Martin de Vos' drawing reveals a confident and fast brushstroke, confirming the dexterity of the artist.

Lead white, $\left(\mathrm{PbCO}_{3}\right)_{2} \cdot \mathrm{Pb}(\mathrm{OH})_{2}$, is identified by $\mathrm{Pb}$ peaks. For yellows both yellow ochre, $\mathrm{Fe}(\mathrm{OH})_{2}$, and lead-tin yellow, $\mathrm{Pb}_{2} \mathrm{SnO}_{4}$, were applied. Red colour was obtained with red ochre, $\mathrm{Fe}_{2} \mathrm{O}_{3}$, and vermilion, $\mathrm{HgS}$, and probably also an organic red colourant. There were two blue pigments found. The most widely used is smalt, $\mathrm{SiO}_{2}+\mathrm{K}_{2} \mathrm{O}+\mathrm{Al}_{2} \mathrm{O}_{3}+\mathrm{CoO}$, which was also the most important pigment in Flemish painting.

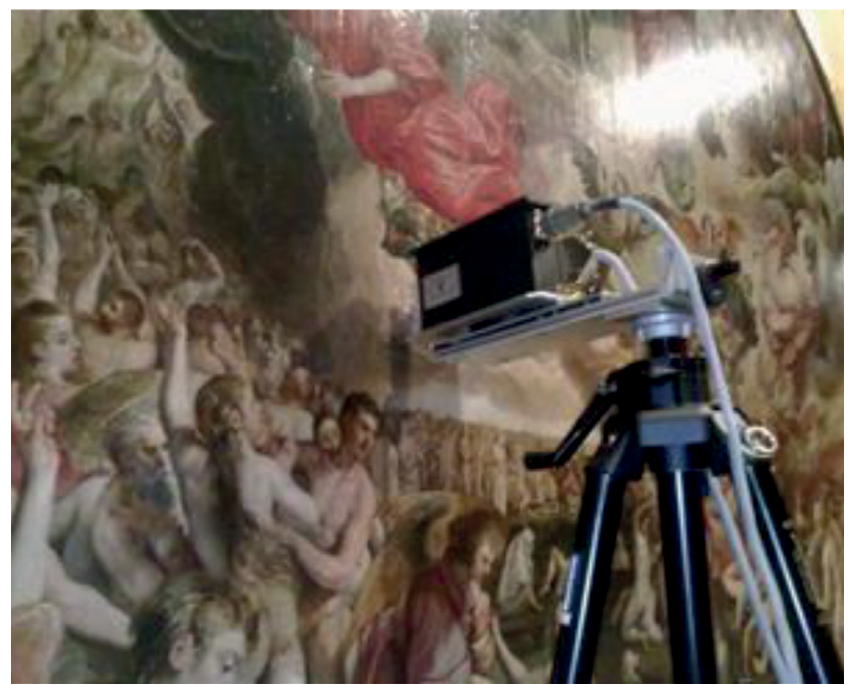

Figure 5.6: Analysis of pigments in situ by a portable XRF device. 


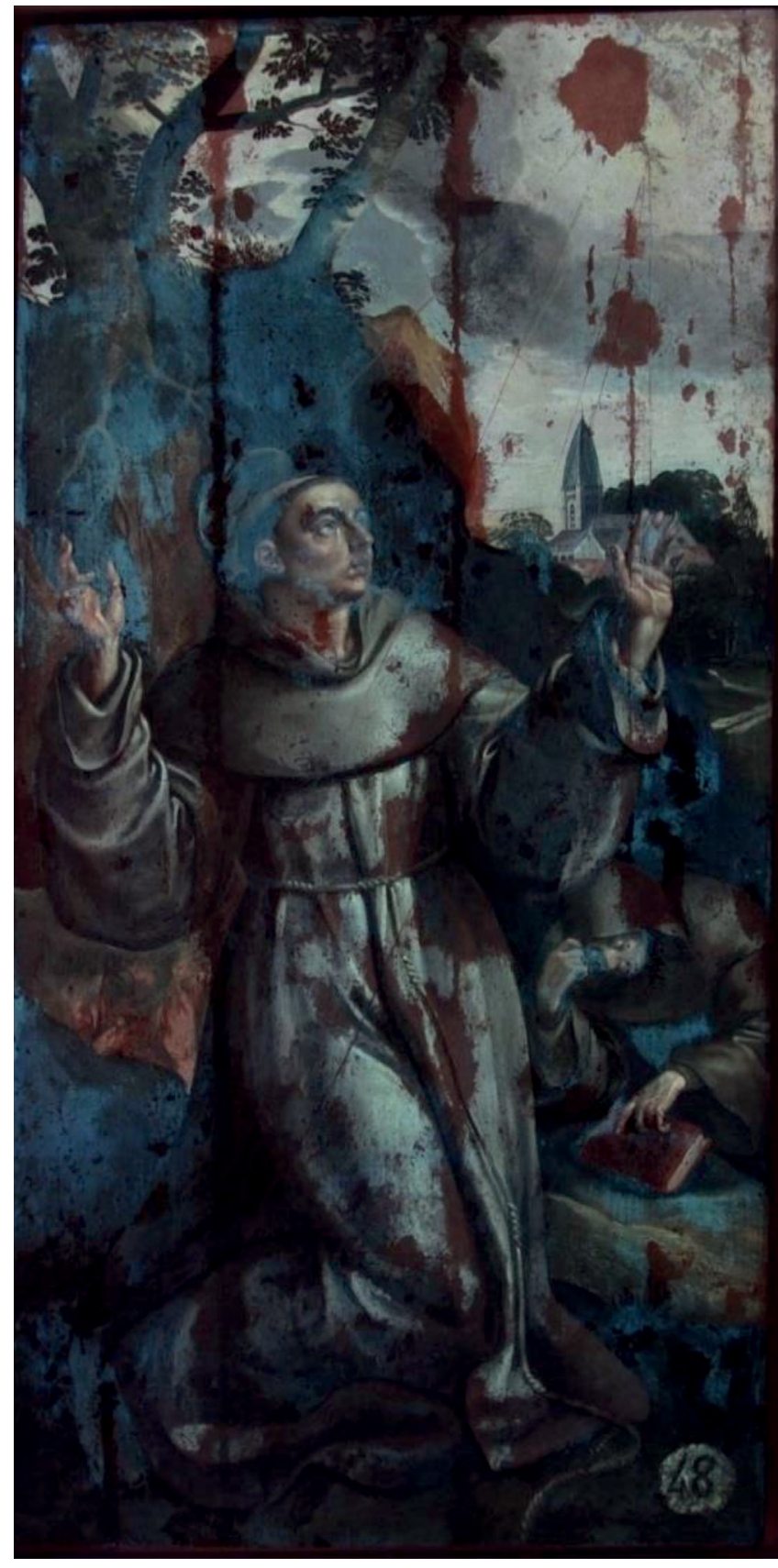

Figure 5.7: With UV light, later retouches can be observed, seen as brownish areas on the painting surface. (St. Francis of Assisi.)

Azurite identified by Cu peaks, $2 \mathrm{CuCO} \cdot \mathrm{Cu}(\mathrm{OH})_{2}$, was applied only for some small details, as it was more expensive than the other blue pigment. Green was obtained with a copper based green pigment, probably copper resinate, $\mathrm{Cu}\left(\mathrm{C}_{19} \mathrm{H}_{29} \mathrm{COO}\right)_{2}$. For shades, brown umber, $\mathrm{Fe}_{2} \mathrm{O}_{3^{\prime}}$ and a bone black, $\mathrm{Ca}_{3}\left(\mathrm{PO}_{4}\right)_{2}+\mathrm{CaCO}_{3}+\mathrm{C}$, were used.

In most cases, two or more pigments were mixed together or overlaid to obtain the desired colour. For example the carnations have lead white $(\mathrm{Pb})$ mixed with smaller amounts of vermilion $(\mathrm{Hg})$, red or yellow ochre $(\mathrm{Fe})$, a copper based green pigment $(\mathrm{Cu})$ and sometimes also lead-tin yellow $(\mathrm{Pb}, \mathrm{Sn})$. Shades of red vestments were made with red ochre $(\mathrm{Fe})$, azurite $(\mathrm{Cu})$, smalt $(\mathrm{Co}, \mathrm{Ni}, \mathrm{As})$ and organic black or red pigment (Ca) on a basic vermilion $(\mathrm{Hg})$ layer. The quantity of pigments in the mixture depends on the colour and tonality that the artist required.

Several points of intervention, or retouching, observed previously with UV light, were analysed using XRF. The results confirm the use of modern pigments such as titanium $\left(\mathrm{TiO}_{2}\right)$ and zinc $(\mathrm{ZnO})$ whites, chrome green $\left(\mathrm{Cr}_{2} \mathrm{O}_{3}\right)$ and cadmium red (CdSe) or yellow (CdS).

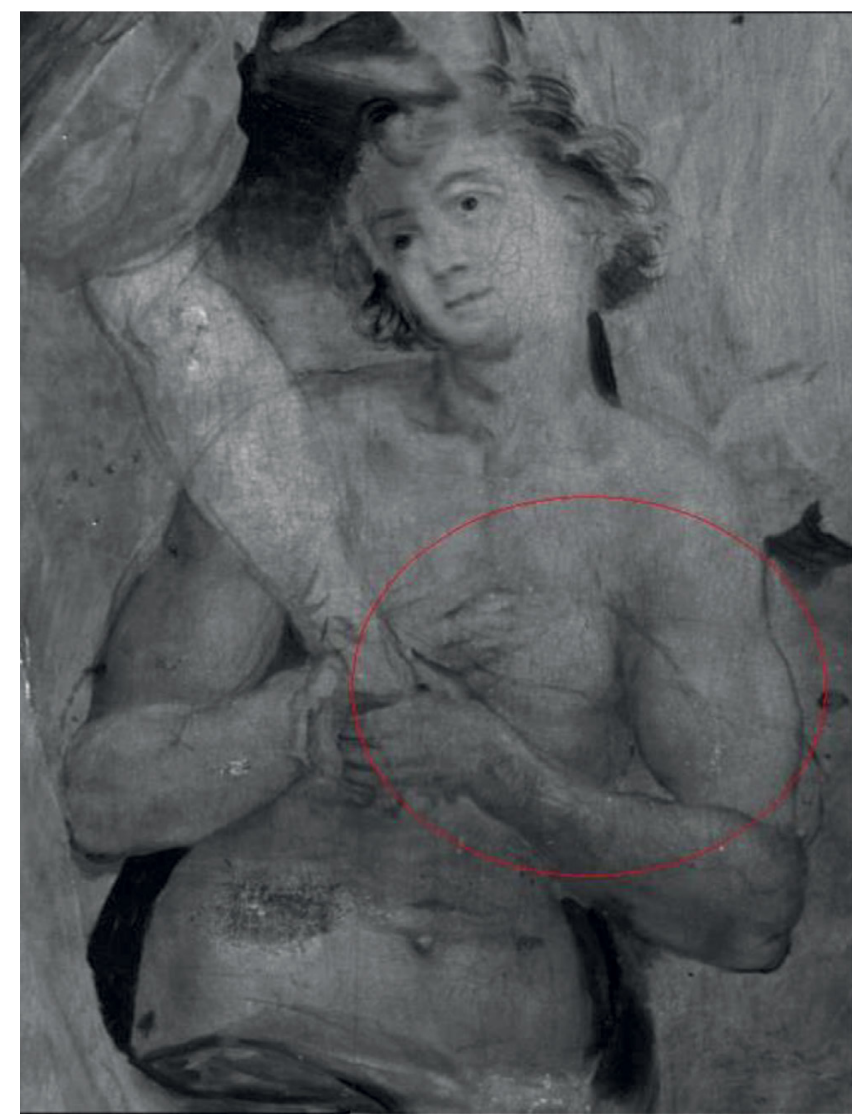

Figure 5.8: Detail of Last Judgement seen with IR reflectography. The underdrawing reveals changes in the position of the arms of the figure.

Although the triptych was restored over wide areas, high quality artistic execution can still be appreciated. The results provided detailed knowledge regarding this special triptych and will help the Museum's restorers with their planned intervention on the St. Augustine panel.

The XRF analysis gave information on preparation layers and the pigments applied. The presence of $\mathrm{Ca}$ and $\mathrm{Pb}$ in every spectrum identifies the existence of preparation, made with gypsum $\left(\mathrm{CaCO}_{4}\right)$ or calcium carbonate $\left(\mathrm{CaCO}_{3}\right)$, while the priming layer was lead white $\left(\left(\mathrm{PbCO}_{3}\right)_{2} \cdot \mathrm{PB}(\mathrm{OH})_{2}\right)$. The painter's palette was the same in all three panels showing pigments common in the $16^{\text {th }} \mathrm{C}$. 


\subsubsection{MANUFACTURING TECHNIQUES OF TARTESIC JEWELLERY}

In recent years, the application of non-destructive physical and chemical methods has allowed significant advances to be made in studies of cultural heritage materials. The archaeometry (applied nuclear physics group) of the CNA (National Accelerator Centre) and various other departments at the University of Seville have used a multidisciplinary approach to study the production processes of ancient gold objects.

The gold elements used to decorate jewellery can carry relevant information about the materials used, the applied heat treatment or the welding/soldering process employed, which is one of the most important ways to characterise gold metalwork. Ancient historical sources, such as Pliny the Elder's Natural History or Leiden Papyrus $X$, have given us accounts of old welding and soldering processes.

Different alloys and soldering processes have been reproduced in order to simulate the manufacturing techniques used in jewellery in the Southern part of the Iberian Peninsula between the $7^{\text {th }}$ and $5^{\text {th }} \mathrm{C}$. BC. The soldered replicas were produced at the CNA laboratories using a protocol based on ancient accounts. The differences between three soldering techniques are reflected in variations in the concentrations of the main elements $(\mathrm{Au}, \mathrm{Ag}$ and $\mathrm{Cu})$ in the soldered area.

Most studies carried out up to now mainly concern objects with a gold content of less than 90 wt.\%. Solders of precious gold work objects, such as some Tartesic jewellery, with high gold concentrations, seem to show different characteristics compared to those with lower gold content. For this reason the CNA research group has systematically studied the manufacturing process of metal objects with alloys containing very high proportions of gold.

A portable micro-XRF set-up was developed to perform in situ non-destructive analysis of objects that

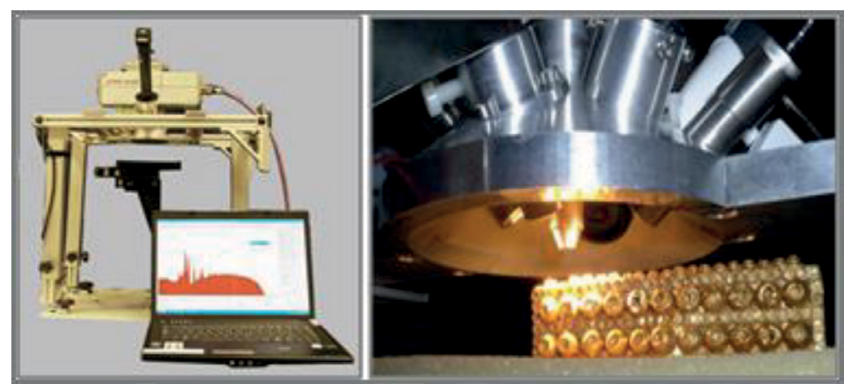

Figure 5.9: In situ micro-XRF analysis of a piece of Tartesic jewelery using portable equipment. cannot easily be moved from museums to a laboratory. This device is equipped with a low power Rh X-ray tube with a polycapillary lens that produces a $30 \mathrm{~mm}$ beam (Figure 5.9).

This system combines high spatial resolution and great sensitivity with portability, enabling identification of soldering techniques for jewellery in exhibition/ storage places.

The study of a material's structure and the production processes used to make metal objects reveal the technological skills of the cultures that created precious decorated artefacts. The scientific study of metals used in hand-crafted products, in particular precious raw materials such as gold, may provide complementary information about ancient trades and the evolution of technology.

Malachite $\left(\mathrm{CuCO}_{3} \cdot \mathrm{Cu}(\mathrm{OH})_{2}\right)$, sodium carbonate $\left(\mathrm{Na}_{2} \mathrm{CO}_{3}\right)$ and acetic acid $\left(\mathrm{CH}_{3} \mathrm{COOH}\right)$ have been used to obtain solid-state diffusion bonding with copper salts. In order to reproduce the ancient decoration patterns and simulate a real case, gold granules were obtained by heating small fragments of gold sheet using a gas welding torch. The granules were then positioned on top of the gold sheet, using a brush impregnated with copper-salt solution, and then heated again. The XRF scanning result across the soldered area shows an increase in copper concentration and a simultaneous decrease of silver and gold content (Figure 5.10).

Three different soldering techniques have been used in old times: solid-state diffusion bonding with copper salts, brazing (hard and soft soldering), and welding without the addition of external material, also known as autogenous welding.

For brazing, a recipe of Pliny the Elder's Natural History was reproduced. This soldering method employs a brazing alloy for joining the two pieces together. This alloy should have a melting point lower than the parts to be connected. Scanning the joining zone highlights a large increase in $\mathrm{Ag}$ and $\mathrm{Cu}$ concentrations and a depletion of Au that occurs at the same time (Figure 5.11).

The resulting composition of the joint differs from that of the primary brazing alloy. In fact during the heating a thin film is formed with an intermediate composition between the compositions of sheet alloy and the brazing alloy. This is a feature that enables the identification of the soldering area, although the original composition of the brazing alloy cannot always be determined. 
Autogenous welding is achieved through local heating by blowing the flame from a welding torch onto the joining area. This type of welding is not easy to carry out, as the temperature cannot be controlled. It therefore demonstrates the great skill of ancient goldsmiths. The

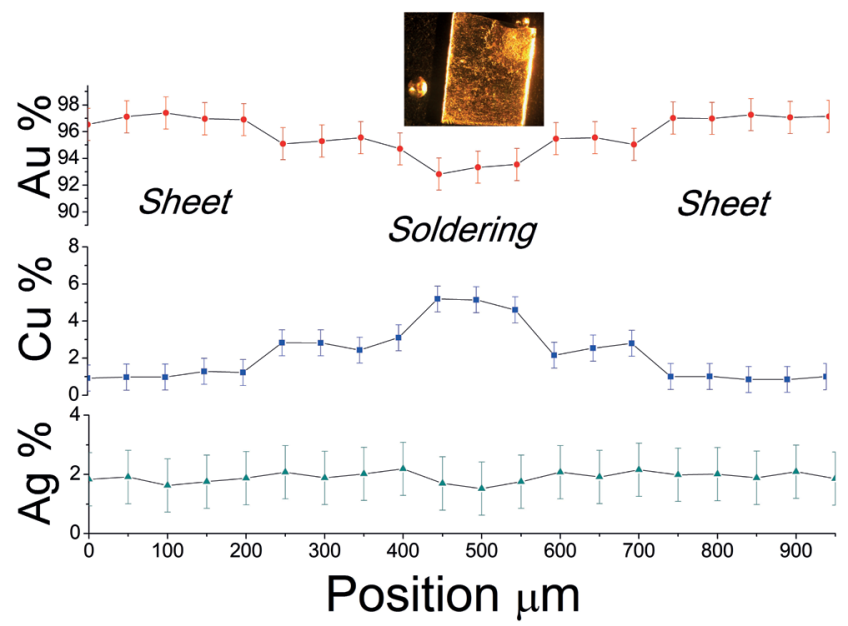

Figure 5.10: XRF scan of diffusion bonding across the copper salt area.

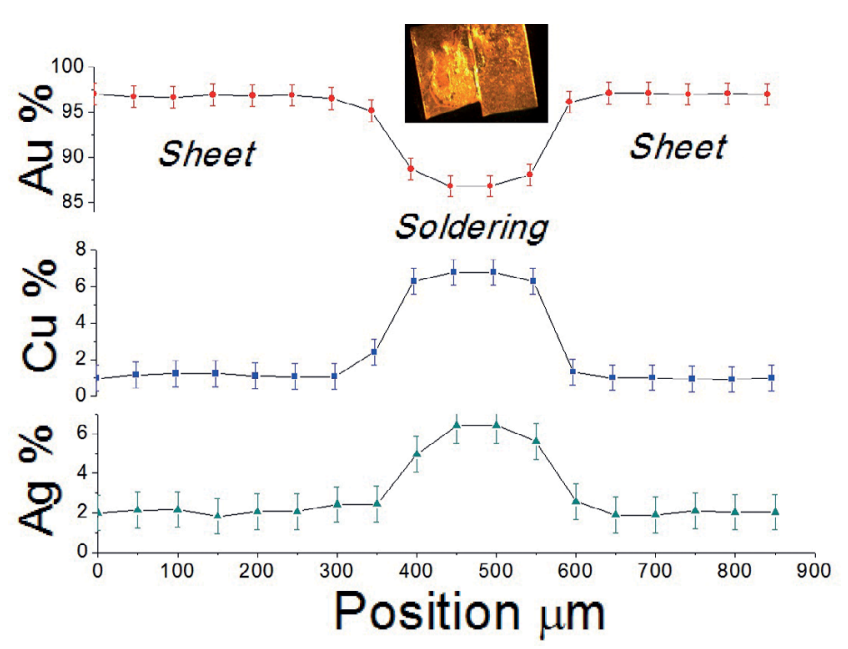

Figure 5.11: XRF scan of the brazing area.

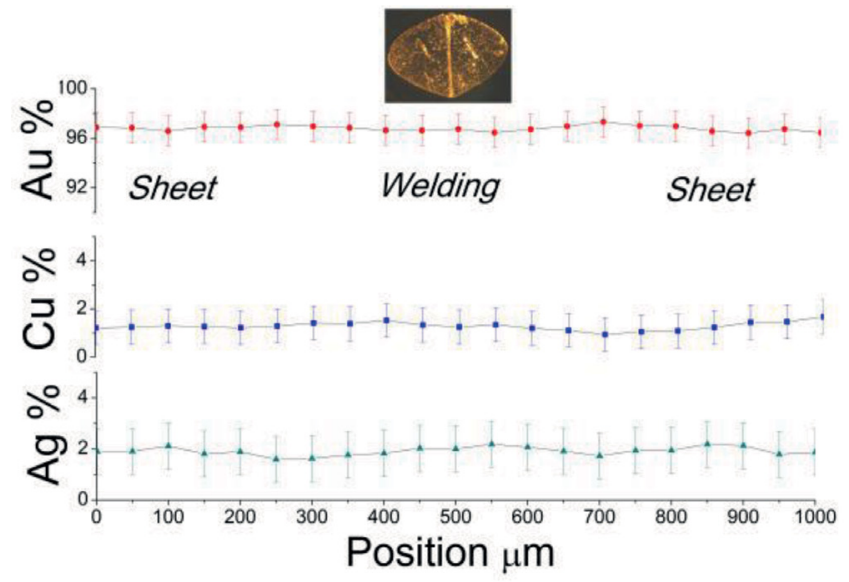

Figure 5.12: XRF scan of the autogenous area. scan of this joining area shows no clear evidence of a change in the alloy composition (Figure 5.12). In fact, this type of welding does not employ any type of soldering material, and consequently the joint has the same composition as the welded pieces.

\subsubsection{NMR STUDIES OF PAINTINGS}

The painting Oil on shaped wood by Lorenzo Lippi (Florence, 1606-1665) (Figure 5.13 inset), is a shaped wooden board painted with angels on both sides. The angel on the front side is painted in colour and holds a candelabrum, while the angel on the reverse side is in monochrome yellow [145-150]. Its front side has been restored by the Opificio delle Pietre Dure (OPD), Florence, Italy. In former times this side has been touched up many times for numerous scratches and paint abrasions. It is very likely that this figure belonged to a series, comprising at least one other angel. The paintings on both sides suggest that the artwork was not meant to hang on a wall but perhaps hung atop an altar or on the top of the partition of a monastery church.

An NMR-MOUSE measurement was arranged at the OPD, Florence. The angel was horizontally positioned with the help of an easel. The points selected for the measurements (Figure 5.13 inset) were decided following consultation with the restorer. The most interesting parts were the knee due to least restoration, the chest because of maximum restoration and the face with no restoration.

A $5 \mathrm{~mm}$ depth range was used with a $17.1 \mathrm{MHz}{ }^{1} \mathrm{H}$ resonance frequency. Each point in the depth profile was measured 512 times and 10 echoes were acquired with a $50 \mu$ s echo time. A $4 \mu$ s long rf pulse with $350 \mathrm{~W}$ was used. The repetition time was $300 \mathrm{~ms}$. A relaxation-weighted spin density was measured with the CPMG pulse sequence. After each measurement the sensor was automatically moved away from the painting by $15 \mu \mathrm{m}$.

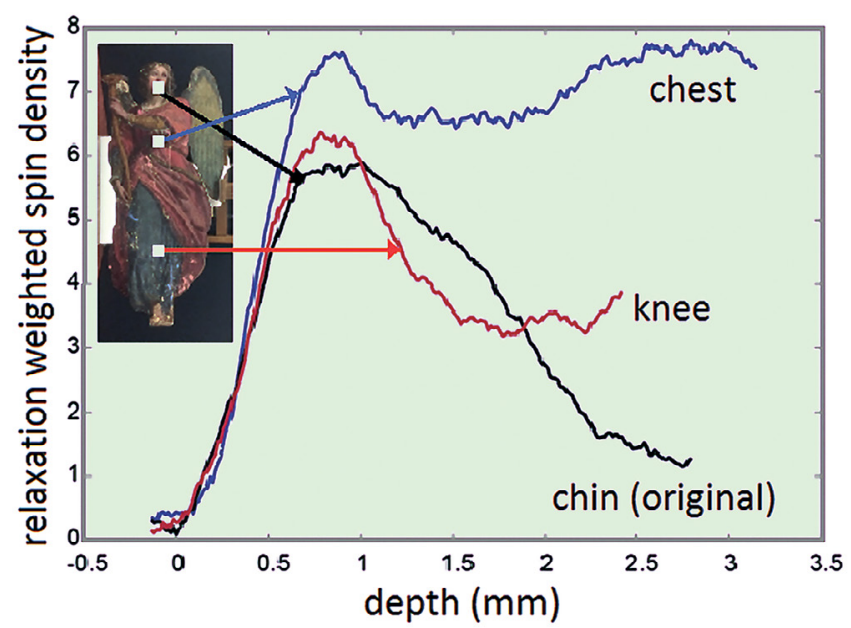

Figure 5.13: NMR studies of Oil on shaped wood by Lorenzo Lippi. Inset shows multi-colour front side of the angel after partial restoration. 1D NMR depth profiles through show the difference between the original chin (black) and the restored chest (blue) and knee (red). The light green spots on the inset mark the three measurement points. 
The sleek look of the two angels and the colour range of the image suggest that it is from Matteo Rosselli. However, certain aspects of the face and the hot colours in the garment cast doubt on its suggested origin. These concern the momentum imparted to the figures when viewed from below and by the act of holding the candelabrum, which is twisted and inflates his clothes, as well as the pathos of the faces and the hot pitch of the outer garment on the front. The strong undeviating characteristics of Rosselli's other works suggest that this cannot be his work. It is therefore considered more reasonable to assign the painting to Lorenzo Lippi, a student of Matteo Rosselli who began to work in close conformity with the model of his Master and soon gained a personal reputation for his own works [152].

Starting at a depth of around $2.5 \mathrm{~mm}$, the measurement was repeated until the sensitive slice reached the sample surface. The depth profile amplitude was calculated from the CPMG echo train envelope as the average over the first 4 echo amplitudes resulting in a relaxation-weighted spin density. A low signal amplitude is received from the old wooden sample because the proton density is very low. Moreover, rapid relaxation of rigid material leads to lower profile amplitudes than slower relaxation from more mobile molecules where the material is more entropy elastic or softer.

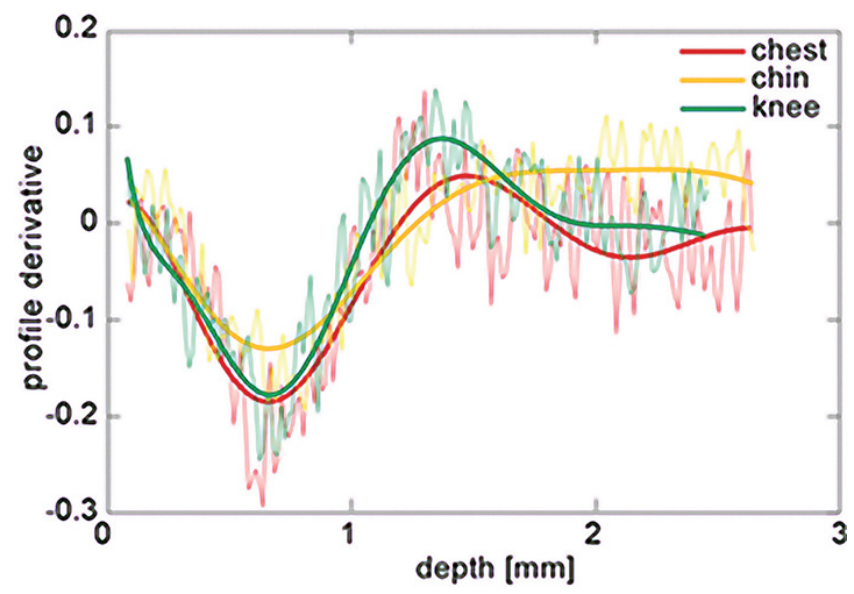

Figure 5.14: Gradients of the depth profiles. The gradient of the original chin is different in amplitude and varies less at the deeper end compared to that of the restored knee and chest.

The face of the angel had not been touched or restored. Interestingly, the signal loss is the most at about $2.5 \mathrm{~mm}$ below the sample surface for the original face region (Figure 5.13). The highest signal is from the chest and it remains high all across the investigated depth range. The knee shows the steepest signal loss (Figure 5.14, green).
The initial peak in all three profiles (Figure $5.13 b$ ) shows the signal from the paint and the base coat which is about $0.5 \mathrm{~mm}$ thick and then the signal drops. The most dramatic drop is seen at the knee after $1 \mathrm{~mm}$ where, according to the restorer, there were considerable interventions.

The fact that there is higher signal at the restored points at large depths indicates that the materials applied during restoration penetrated quite deeply into the wood. It is interesting to note that the gradients (Figure 5.14) of the signals from the touched-up points are similar. They are more negative in the beginning and then 'wavy' for both chest and knee, which may indicate restoration agents having diffused into the sample, while the profile gradient tappers slowly for the original (Figure 5.13, yellow) and is constant at depths greater than $1.5 \mathrm{~mm}$. The measurements support the suggestion that a minimal quantity of restoration materials should be applied so that the materials beyond the varnish and the paint are least affected.

\subsubsection{TRADITIONAL SINGLE POINT XRF ANALYSIS FOR CULTURAL HERITAGE DIAGNOSTICS}

X-Ray Fluorescence (XRF) is one of the most used diagnostic techniques in the field of Cultural Heritage $[154,155]$, thanks to its many favourable aspects, such as the possibility of carrying out quick (only a few minutes per spectrum) multi-elemental, non-destructive/ non-invasive analyses. Moreover, the portability of the instrumentation allows in situ measurements to be carried out in the museum or in the restoration laboratory.

As XRF is a completely non-destructive/noninvasive, multi-elemental, in-situ analysis, it is one of the most widespread techniques in the field of cultural heritage diagnostics and is particularly suitable for analysis of the composition of materials.

Detecting particular elements allows the material composition of art objects, such as pigments, to be determined. The main limitations of this type of portable spectrometer, which works at atmospheric pressure, are the lack of information about molecules and its inability to provide information on elements lighter than sodium. Thus XRF needs to be complemented by other diagnostic techniques in order to obtain a complete characterisation of a work of art.

The LABEC laboratory of the National Institute of Nuclear Physics (INFN) in Florence is a facility dedicated to Cultural Heritage and Environmental studies [156]. In 2011 an XRF spectrometer was developed which was especially customized for Cultural Heritage diagnostics [157]; see Figure 5.15. This spectrometer has a high sensitivity for 


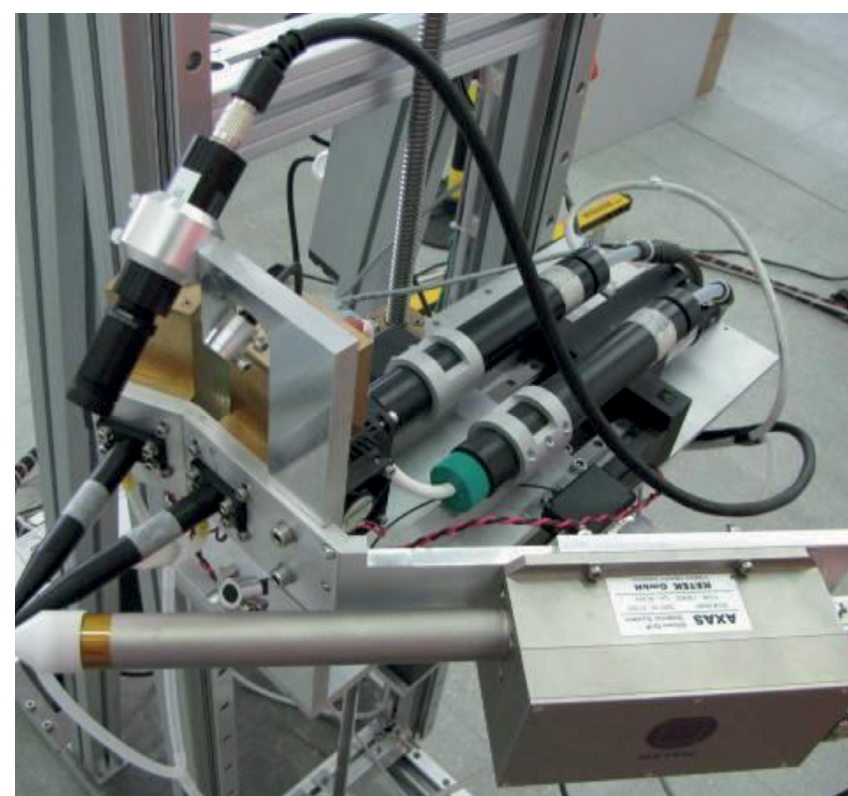

Figure 5.15: The LABEC XRF measuring head.

low- $Z$ elements, because the production and detection efficiencies of low-energy $X$-rays are enhanced by a $\mathrm{He}$ flow along the $X$-ray paths from the source to the sample and from the sample to the detector.

The efficiency is maximised over a wide range of $X$-ray energies by exploiting tubes with different anodes. In the measurements described below we used tubes with molybdenum and tungsten anodes, equipped with $125 \mu \mathrm{m}$ thick Beryllium windows. The collimator gives a spot with a diameter of 1 millimetre. Each spectrum was collected over $120 \mathrm{~s}$ at $25 \mathrm{kV}$ (or in some cases at $38 \mathrm{kV}$ for tin detection) with a current of $0.3 \mathrm{~mA}$ and analysed using WinQXAs software.

The entire system, including the measuring head and the translation stages, is remotely controlled. The distance between the spectrometer and the controller is limited only by the length of the $6 \mathrm{~m}$ connecting cables. This distance allows safe operation and measurement even when the artwork is not easily accessible, e.g. in this case, where the painted surface extends up to some metres above the floor and it is necessary to mount the spectrometer on scaffolding.

LABEC spectrometer - High sensitivity at low energies obtained through:

- the use of non-contemporary multiple-X-ray tubes using anodes of different materials;

- Helium atmosphere in the path of the X-rays.

This instrument has been successfully used for many case studies in the field of Cultural Heritage diagnostics [157-159]. Here we show one of the most interesting applications, a study of the frescoes by Beato Angelico.

\section{THE FRESCOES BY BEATO ANGELICO}

Beato Angelico (1400-1450) is an Italian painter and one of his most famous masterpieces is the fresco cycle, painted between 1438 and 1445, in the monastery of San Marco in Florence. Two frescoes, the Annunciazione (see Figure 5.16) and the Madonna delle Ombre (see Figure 5.17) have been analysed by XRF.

Beato Angelico mainly used the buon fresco technique in which paint is applied to the freshly laid plaster and the pigment is dispersed using water. In contrast, in the secco technique, the pigment is tempered in an organic medium (such as egg yolk or whole egg) and then applied to the dried plaster. These two painting techniques make use of different pigment types. For instance, lead-based pigments become dark if employed in the fresco technique, due to basic environment created by calcium carbonate in the plaster. Therefore lead-based pigments can only be used with the secco technique.

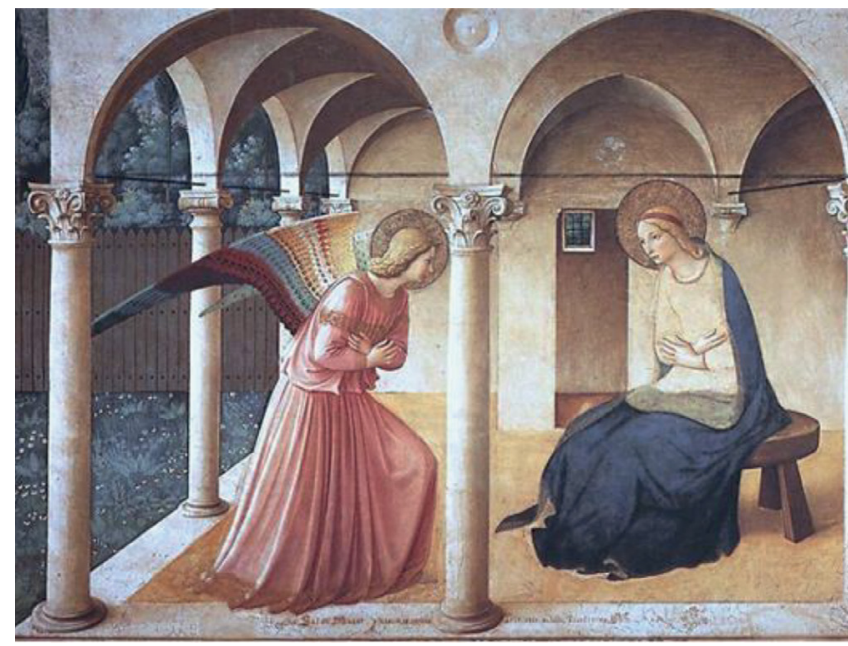

Figure 5.16: Annunciazione, by Beato Angelico, in Museo di San Marco, Florence, Italy.

Figure 5.17: The Madonna delle Ombre, by Beato Angelico, in Museo di San Marco, Florence, Italy

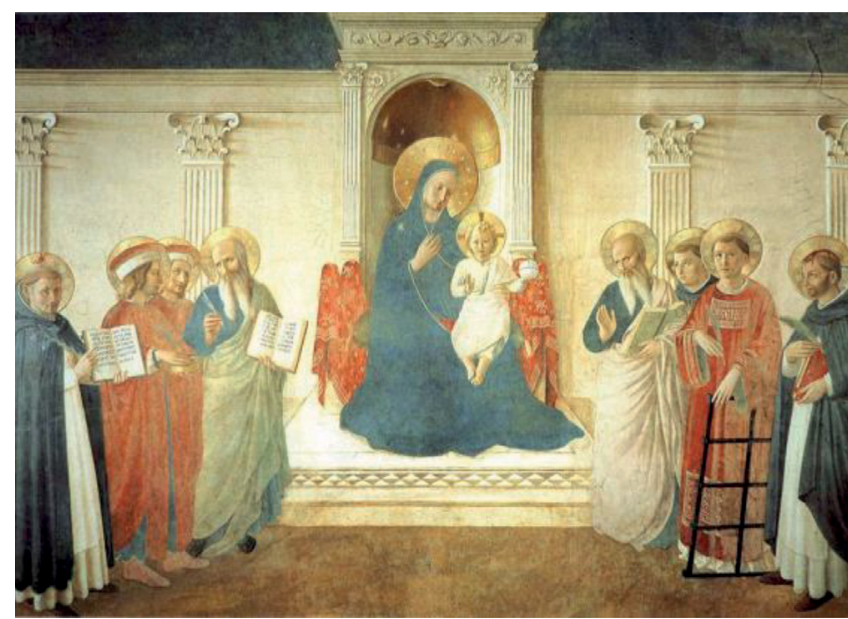


The Madonna delle Ombre is an exception in the San Marco cycle because it is made with a mixture of both the fresco and secco painting techniques. This feature makes this mural of particular interest as it allows a direct comparison of the two techniques applied by the same artist. These two different painting techniques, which require different kinds of pigments, can be easily characterised by XRF analyses.

\section{PRELIMINARY CONSIDERATIONS FOR XRF ANALYSES}

As is well known, the study of a mural painting is a challenging task for many reasons, the most important of which are: - The presence of plaster, pigments, degradation products, pollution and restorations provides a huge amount of information which is difficult to separate, as it is not trivial to clearly link a particular element to the corresponding material.

- The same element may have different origins, increasing the ambiguity of the material characterisation. For example, $\mathrm{Ca}$ and $\mathrm{S}$, two elements always present in the plaster, are also involved in sulfation, i.e. the formation of gypsum $\left(\mathrm{CaSO}_{4} \cdot \mathrm{H}_{2} \mathrm{O}\right)$.

- In spectral analysis, the presence of so many elements gives rise to problems de-convoluting the X-ray lines required for element identification.

\section{CHARACTERISATION OF THE PAINTING PALETTE AND TECHNIQUE}

The fresco palette of Beato Angelico is not so varied because he used only poor pigments, mainly iron-based pigments or earths. Many types of earth pigments exist, from yellow to red, brown and black, depending on the quantity and quality of impurities. A high percentage of pyrolusite $\left(\mathrm{MnO}_{2}\right)$, for example, gives dark shades as in the case of umber, while the prevalence of hematite $\left(\mathrm{Fe}_{2} \mathrm{O}_{3}\right)$ gives the well-known brick-red hue. Also the composition of green earths is similar, but the content of glauconite (Fe K phyllosilicate) and celadonite (phyllosilicate of $\mathrm{K}, \mathrm{Fe}$-in both oxidation states-, Al and hydroxide) produces different hues $[160,161]$.

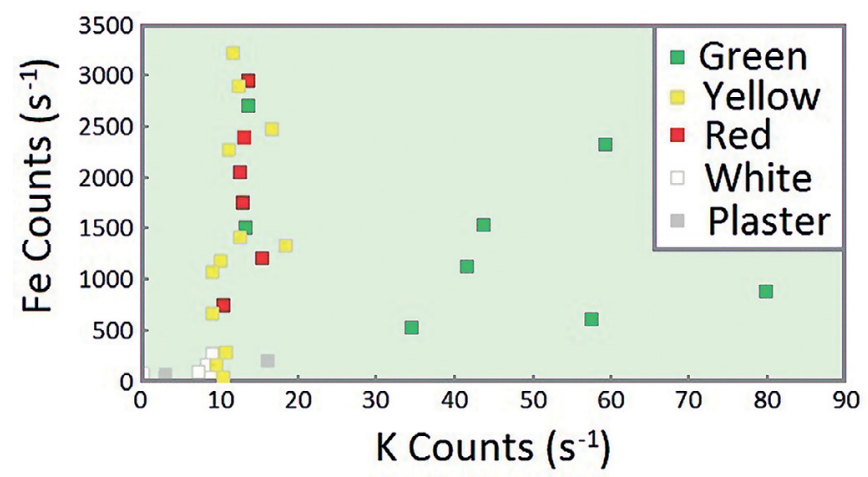

Figure 5.18: Iron vs. potassium counts in the Annunciazione (counts/sec); error bars are smaller than the symbols.
Among all the earth varieties described above, by far the most abundant element is iron. The only way to discriminate different earths is to study the trace elements, such as for example the lower- $\mathrm{K}$ K, Si, Mg and $\mathrm{Al}$ contributions which are the most useful in our case, and also $\mathrm{Zn}$ or Ti. In this way it is possible to characterise pigments and, in the most favourable cases, to point out the use of a mixture of earths.

Two examples from the Annunciazione are reported in Figures 5.18 and 5.19. Here different earths, presenting different colours (green, yellow and red), are compared with white areas and the plaster. In figure 5.18 we compare the $\mathrm{K}$ counts with $\mathrm{Fe}$, while in figure 5.19 we compare the Zn counts with Fe.

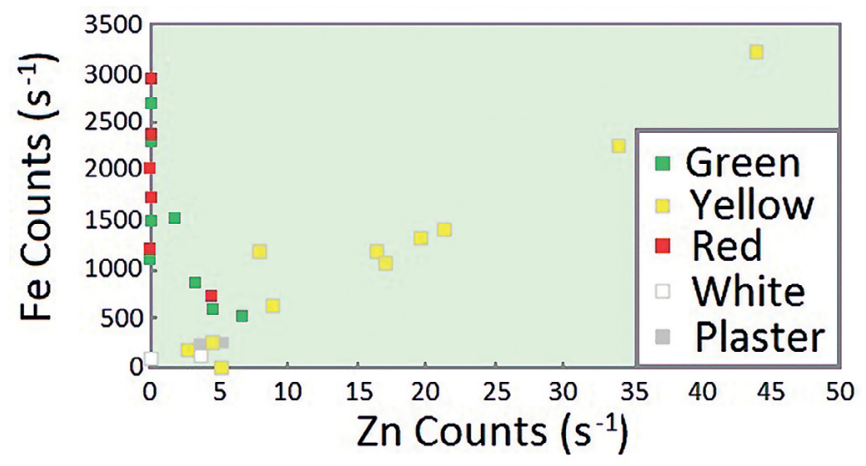

Figure 5.19: Iron vs. zinc counts in the Annunciazione (counts/ $\mathrm{sec})$; error bars are smaller than the symbols.

Figure 5.18 shows that green earths generally have large quantities of potassium; this suggests that the two green points with a $\mathrm{K}$ concentration comparable with those of the other pigments, correspond to an area painted not with a green earth but with a mixture of other earths. The dispersion of $\mathrm{K}$ counts is due to the variable percentage of the K-containing minerals (glauconite and celadonite).

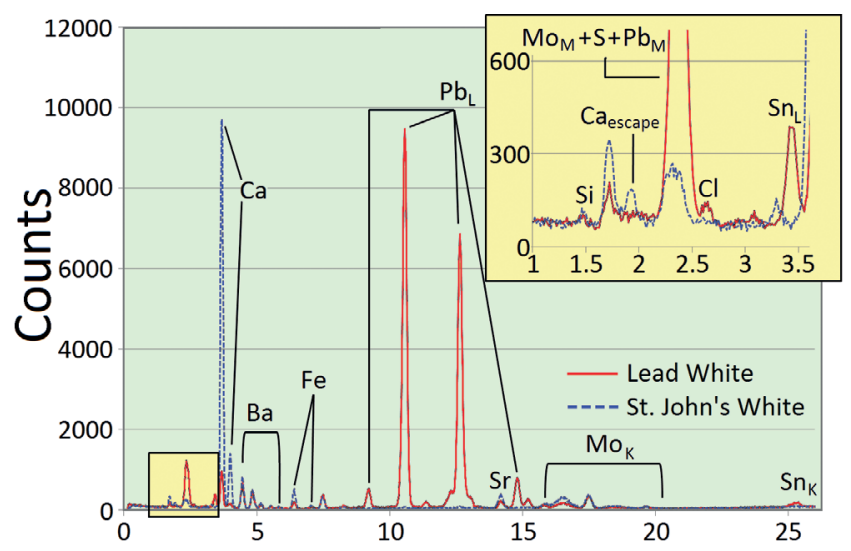

Figure 5.20: Comparison of XRF spectra from two white areas of the Madonna delle Ombre (red solid line) and the Annunciazione (blue dashed line). The inset shows large differences between the two paintings in the $X$-ray region below $3.5 \mathrm{keV}$. The spectra obtained from both paintings are comparable because the experimental set-up was exactly the same, including the sampling time, current and voltage. 
Figure 5.19 clearly shows that the yellow earths are characterised by $\mathrm{Zn}$ traces, not present in the other pigments. In this case it is possible to recognize the use of mixtures: the presence of $\mathrm{Zn}$ in green and red areas suggests that the yellow earth was added in order to achieve a different hue.

As mentioned previously, the Madonna delle Ombre is mainly made with the secco technique. We found, indeed, the characteristic elements of the secco technique, as $\mathrm{Hg}$, $\mathrm{Pb}, \mathrm{Sn}$ and $\mathrm{Cu}$, supposedly for vermillion, lead white, tin yellow and azurite/malachite respectively.

Figure 5.20 shows the XRF comparison between two white areas in different paintings, which are painted with different pigments: the Saint John white (calcium carbonate $\mathrm{CaCO}_{3}$ ) from the Annunciazione, and lead white from the Madonna delle Ombre.

The sampled area of the Annunciazione was found to be composed mainly of St. John's white (perhaps with small amounts of earths), as mainly $\mathrm{Ca}$ and elements characteristic of the plaster were detected. In contrast, the points of analysis in the Madonna delle Ombre clearly show the presence of lead, indicating the use of lead white and some small amounts of lead-tin yellow.

The use of a working voltage of $38 \mathrm{kV}$ permitted the tin $\mathrm{K}$-lines to be revealed. This is not possible with the more common lower voltage $30 \mathrm{kV}$ tubes. This feature is crucial for Sn identification when $\mathrm{Ca}$ and $\mathrm{K}$ are also present, since the Sn L-lines ( $3.44 \mathrm{keV}$ ) overlap with the $\mathrm{K}$ and $\mathrm{Ca} \mathrm{K}$-lines (3.31 keV and $3.69 \mathrm{keV}$, respectively).

\subsubsection{XRF IMAGING}

Non-invasive in-situ analyses are strongly preferred for studies in the Cultural Heritage field. X-ray Fluorescence (XRF) is one of the most widespread techniques for noninvasive compositional characterisations [162-164].

Most objects of artistic or historical interest have quite inhomogeneous structures, even within apparently uniform areas. "Anomalous" details may be present, even on small scales down to a hundred microns or less. Such non-homogeneous texture can become problematic for compositional analysis, since these features are not easily identified by visual examination [165], and "spot" analysis can result in misleading information on the composition of the material under study (see for example [157-159]).

In order to overcome the risk of misleading or ambiguous information, a portable XRF scanner was developed. The measuring head is coupled to three linear axis stages (Figure 5.21), allowing information to be acquired over a large area

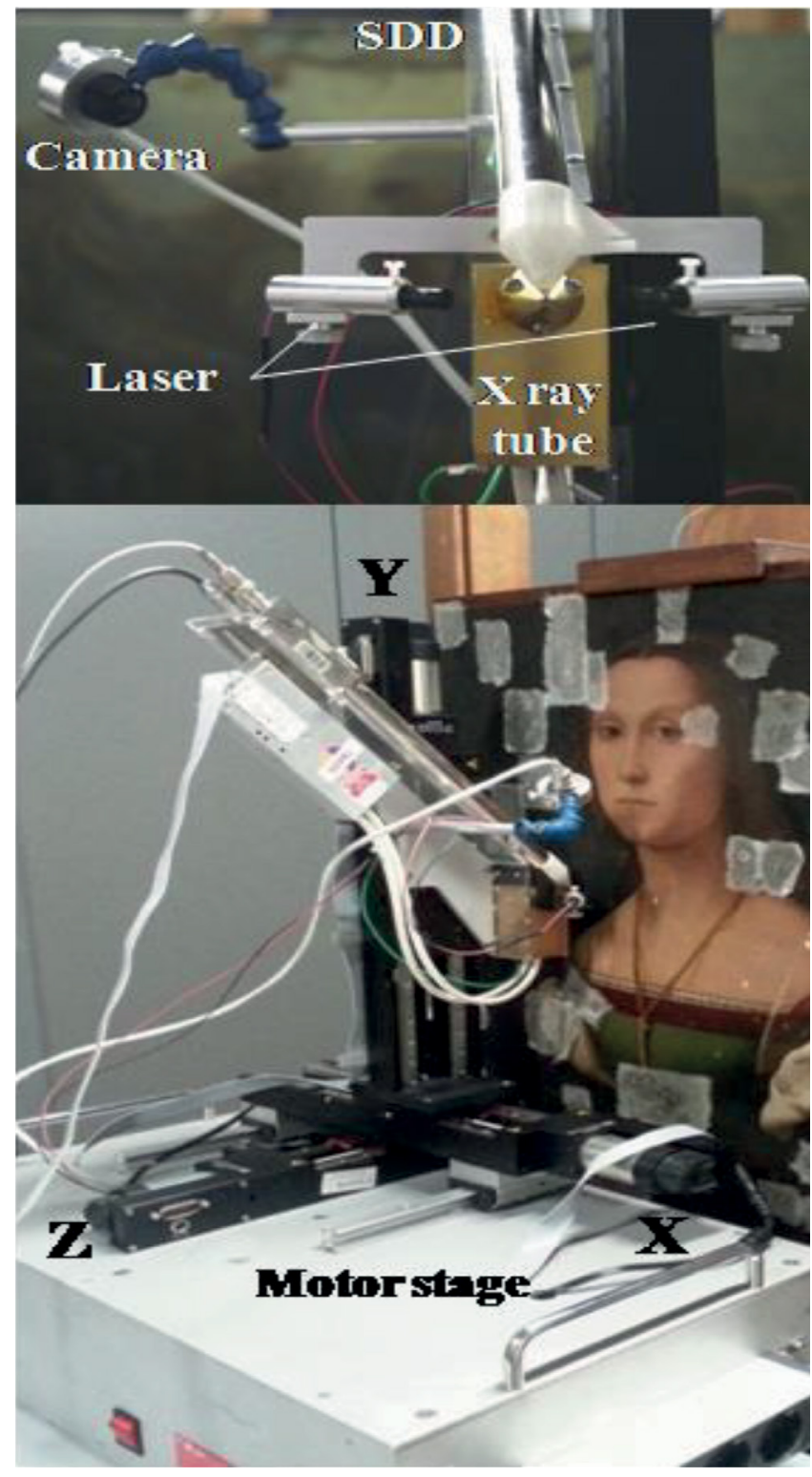

Figure 5.21: The XRF scanner (bottom) and a blow up of the measuring head (top), mounted on the three axis motor stages. The picture was taken during measurements on Raffaello's painting La Muta.

up to $20 \times 20 \mathrm{~cm}^{2}$. Both the spectra and the corresponding spatial coordinates are recorded at the same time, permitting distribution maps of elements to be obtained. This system is easily adapted for diverse cultural heritage applications.

Here we report a few examples illustrating the immediacy and the effectiveness of the imaging XRF.

The first example looks at the painting palette of Sandro Botticelli in the fresco Sant'Agostino nello Studio (See Figure 5.22).

The Fe and $\mathrm{K}$ spatial distributions, shown in figure 5.22, were interpreted as an indication of the use of ochre (iron oxide) $[161,166]$ for the yellow/orange and green areas, while the increase of $\mathrm{K}$ in the book holder was explained assuming the use of a green earth (typically obtained by K-rich minerals) 
A key feature provided by XRF imaging is the possibility of presenting information on an analysed area by studying and comparing elemental maps from that area. This approach reduces the number of single spot analyses needed, saving time for both data acquisition and analysis.

The $\mathrm{Cu}$ and $\mathrm{Pb}$ maps suggest the use of green copper based [167] and white lead [168] pigments. The presence of $\mathrm{Cu}$ in a small area of the book holder, which is instead mainly made with green ochre, suggests the presence of a restoration. This idea is supported also by the $\mathrm{Pb}$ map, which points out an increase of both $\mathrm{Pb}$ and $\mathrm{Cu}$ in that area. Lead white was not used with the fresco technique, as it is unstable in presence of water and tends to become brownish [169], so it must have been added later.

The second example deals with the panel painting Crocifissione by Simone Martini (Figure 5.23). Pb and Zn maps overlap in a small portion of the studied white area. This feature points out the presence of the lead white pigment, typically used for glazing (as the Christ's loincloth), and the zinc white pigment, which appeared on artists' palettes after the middle of $18^{\text {th }} \mathrm{c}$. [170].

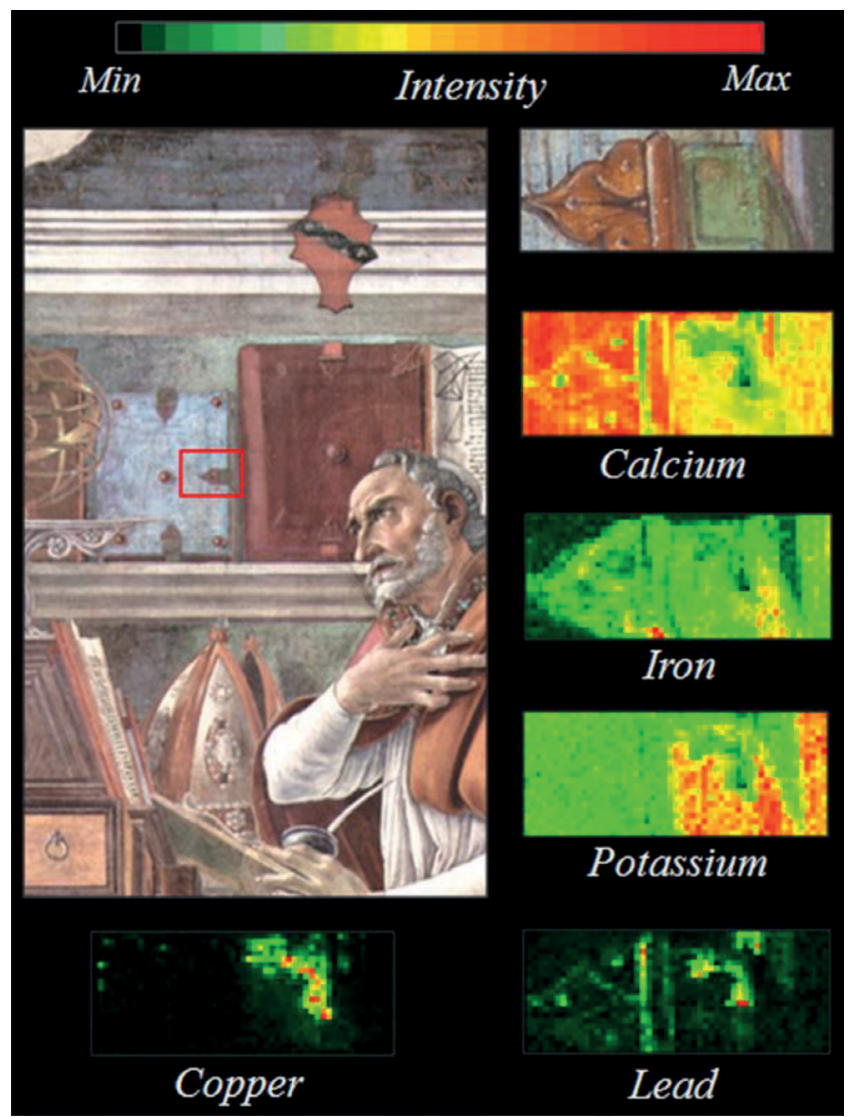

Figure 5.22: Sandro Botticelli's painting: Sant'Agostino nello studio. The red box indicates the detailed area studied by XRF and the smaller panels show the corresponding elemental maps obtained. Measurement conditions: $5.5 \times 2 \mathrm{~cm}^{2}$ area, $1 \mathrm{~mm} / \mathrm{s}, 20 \mathrm{kV}, 100 \mu \mathrm{A}, 18 \mathrm{~min}$ acquisition time.

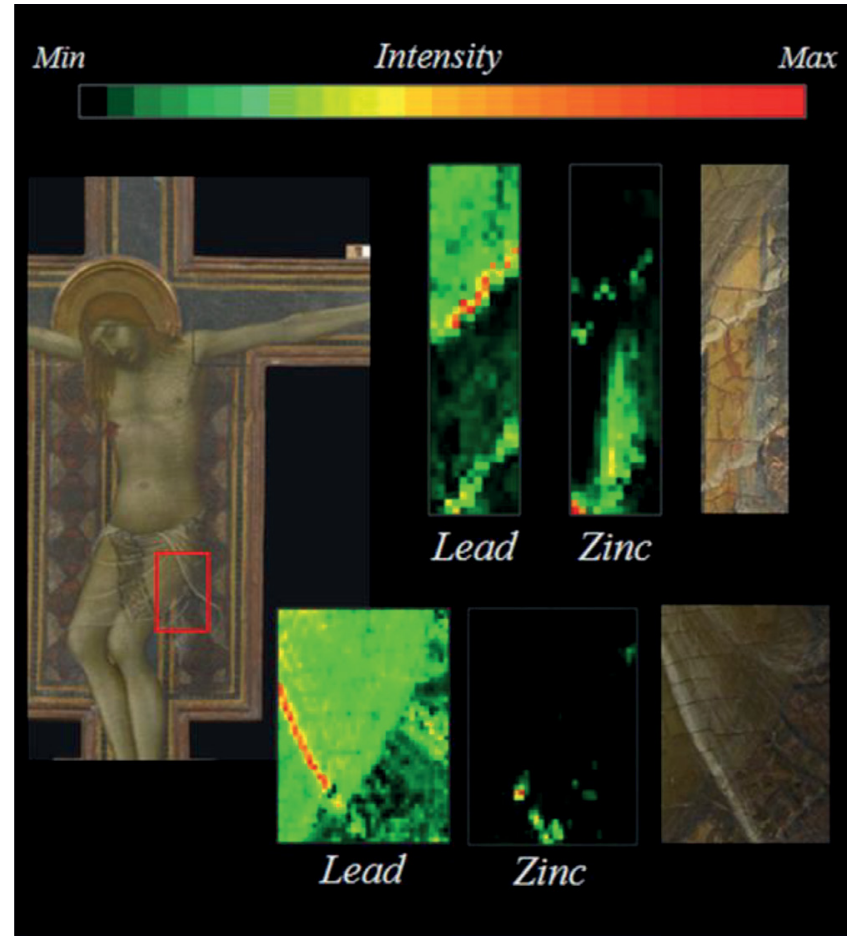

Figure 5.23: Simone Martini, Crocifissione, and the details of Christ's loincloth. Measurement conditions: $1 \mathrm{~mm} / \mathrm{s}$, $35 \mathrm{kV}, 100 \mu \mathrm{A}$. Area 1: $5 \times 3 \mathrm{~cm}^{2}(25 \mathrm{~min})$; area $2: 5 \times 4 \mathrm{~cm}^{2}$ (33 min. acqusistion time)

While the former is compatible with the original pigments, the latter is clearly connected to a restoration. The $\mathrm{Pb}$ and $\mathrm{Zn}$ maps allow the identification of the transition from the lead white, reasonably original, to the zinc white, a modern retouching.

The third example studies a pentimento in the Raffaello's La Muta [171]. This is an underlying image in the painting which is evidence of revision by the artist.

In area 1 of figure 5.24 (element maps shown at the top of the figure), the Cu distribution indicates two green ribbons, which were painted with a copper based pigment. The upper one is not visible by eye, as it has been overpainted with a red colour characterised by $\mathrm{Hg}$, which indicates the use of cinnabar [172]. The IR reflectography [173] of area 1 also highlights the presence of both ribbons.

Area 2 of figure 5.24 contains the bow of the bodice (element maps shown at the bottom of the figure). In this case IR reflectography could identify the second bow, since the pentimento is painted in a black area, where IR is blind.

Conversely, XRF imaging allowed the presence of the pentimenti to be deduced. The two bows are clearly visible in the $\mathrm{Hg}$ map, suggesting the use of cinnabar for the red colour.

The final application in this section is a good example of how XRF scanning allows for different approaches to a study. This study started with a reconnaissance of the area to investigate and then focused on details of interest, discovered thanks to the first, quick reconnaissance. This procedure, only possible with an imaging approach, 


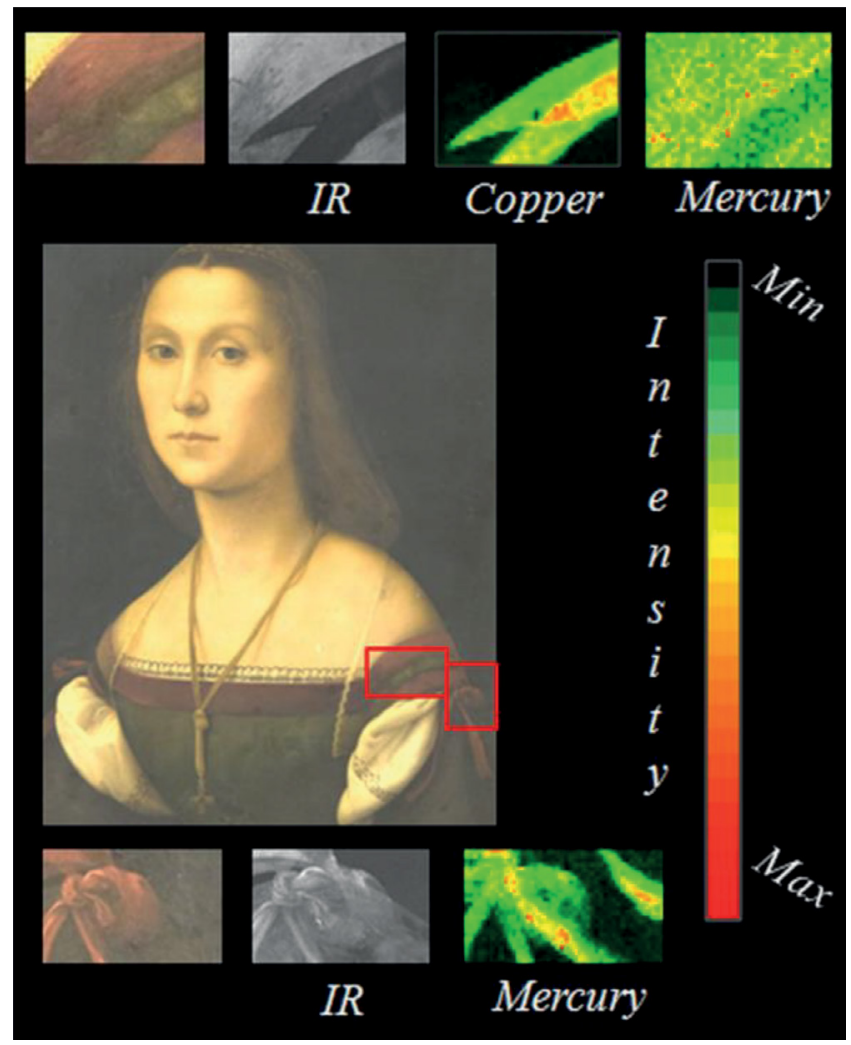

Figure 5.24: Raffaello's La Muta and the details of pentimenti. Measurement conditions: $1 \mathrm{~mm} / \mathrm{s}, 35 \mathrm{kV}, 100 \mu \mathrm{A}$. Area 1 (left): $5 \times 3 \mathrm{~cm}^{2}(25 \mathrm{~min})$; area 2 (right): $5.5 \times 2.5 \mathrm{~cm}^{2}(23 \mathrm{~min}$. acquisition time).

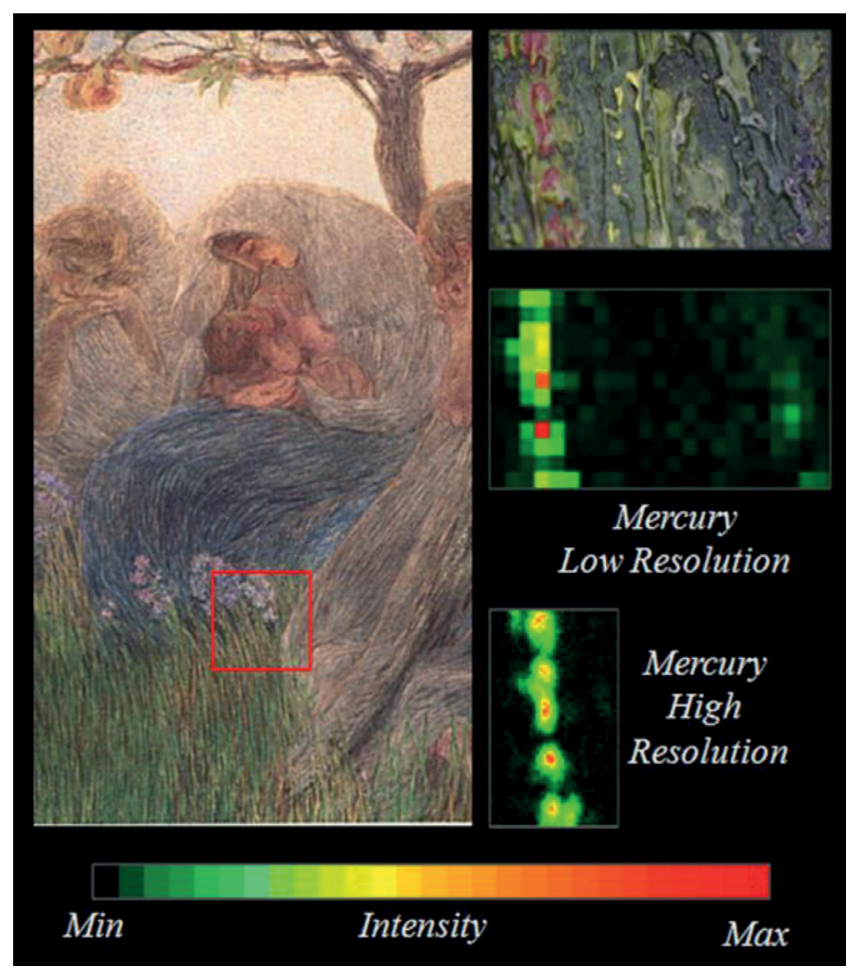

Figure 5.25: Gaetano Previati, La Maternità, with a blow up of the grass detail. Experimental conditions: $30 \mathrm{kV}, 100 \mu \mathrm{A}$. $\mathrm{Hg}$ maps were acquired at different resolutions. Top (low res.): $1 \mathrm{~mm} / \mathrm{s}, 2.3 \times 1.2 \mathrm{~cm}^{2}$ (4.6 min.); Bottom (high res.): $0.5 \mathrm{~mm} / \mathrm{s}$, $0.8 \times 1.2 \mathrm{~cm}^{2}(12.8 \mathrm{~min}$. acquisition time). proved to be very useful in the study of painting palette used by Gaetano Previati in La Maternità.

Previati was a Divisionist, thus he combined the colours optically on the canvas by simply juxtaposing them, without physically mixing pigments on the palette $[174,175]$. With this technique, pigment composition changes on millimetre scale, as shown in figure 5.25.

The red pigment was identified as cinnabar, and the use of pigments in separate, adjacent regions was observed. This confirmed what Previati wrote in his notes about his use of colours. Also the shape of a single brush stroke is evidenced by the element maps.

\subsubsection{THE SNEAKY TITANIUM}

Although the detailed knowledge of the palette of a painter could certainly be very important and useful for art historians and restorers, the most frequent question from the public, whether a particular painting is authentic or a forgery, cannot be answered in general on the basis

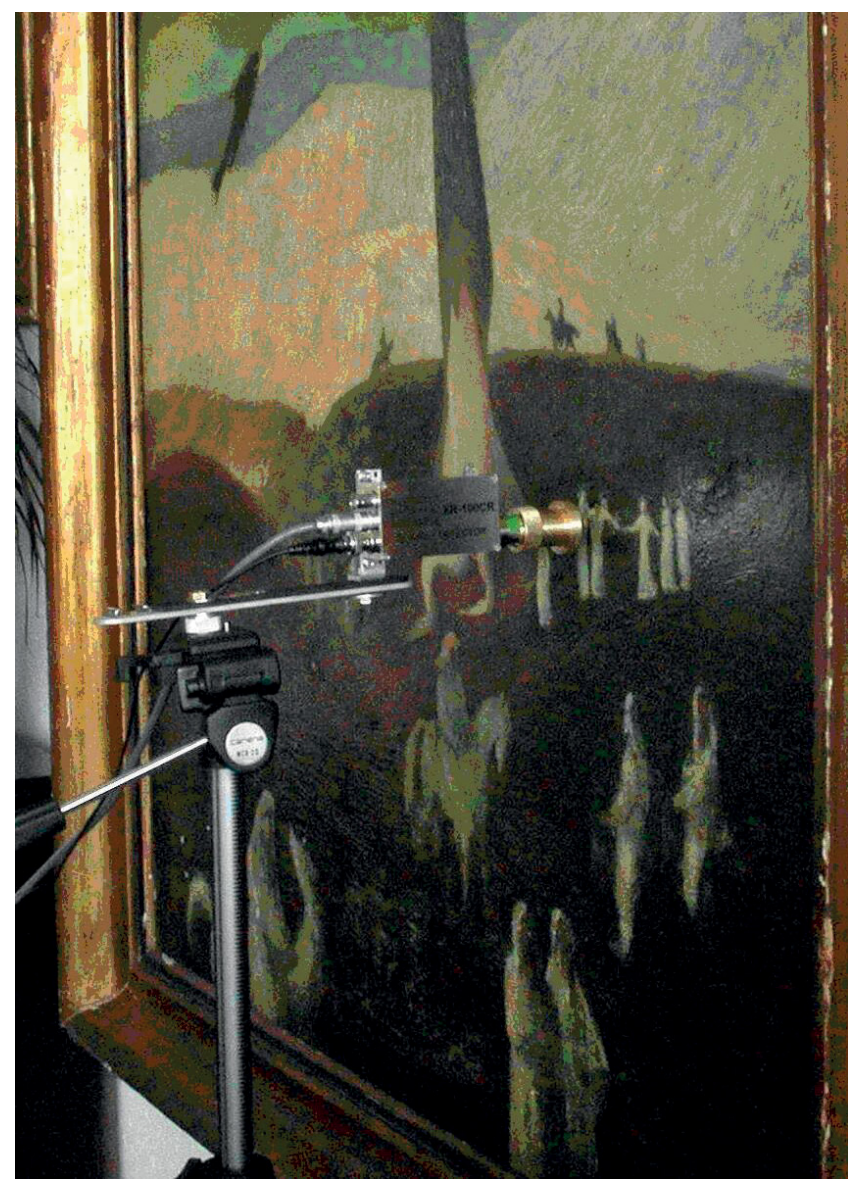

Figure 5.26 shows The third cedar, a painting attributed to Tivadar Csontváry Kosztka (1853-1919). Serious doubts have been raised about its originality. However, the opinion amongst experts was not unanimous. One skeptic expert referred to the sometime detection of $\mathrm{Ti}$ in the paints, but no documentation of this observation was presented. In order to justify or reject this opinion an in situ XRF analysis was commissioned. 
of the elemental composition. There are, however, a few special cases, when the presence of a particular element provides unambiguous evidence for the possible age of the painted spot. The most well-known example is the presence of titanium in white coloured spots. As titanium white $\left(\mathrm{TiO}_{2}\right)$ has only been available since around 1920 , its presence in a painting by an artist who lived and worked before this date provides indisputable indication for either forgery or later repainting [176].

This Ti method was successfully used to distinguish two forgeries previously assigned as genuine paintings by Géza Mészöly (1844-1887). In all the selected white points of the suspicious paintings a strong titanium $\mathrm{K}_{\alpha}-\mathrm{K}_{\beta}$ doublet dominated the radioisotope-excited XRF spectrum, while no traces of Ti were observed in the $X$-ray spectrum of an authentic Mészöly painting. This result has provided a strong scientific argument to decide that the paintings in question were fakes. In addition, the strong detected $\mathrm{Pb}$ L-lines supported the assumption that Géza Mészöly used lead or Chremnitz white $\left(2 \mathrm{PbCO}_{3}\right.$ or $\left.\mathrm{Pb}(\mathrm{OH})_{2}\right)$.
Figure 5.26 shows The third cedar, a painting attributed to Tivadar Csontváry Kosztka (1853-1919). Serious doubts have been raised about its originality. However, the opinion amongst experts was not unanimous. One skeptic expert referred to the sometime detection of $\mathrm{Ti}$ in the paints, but no documentation of this observation was presented. In order to justify or reject this opinion an in situ XRF analysis was commissioned.

Although at first sight it appeared that there was some Ti present in the paint, a more detailed analysis of the $X$-ray spectrum clearly showed that the observed peaks did not come from Ti but rather belonged to the $L X$-ray multiplet of $\mathrm{Ba}$. (The Ti-K $\mathrm{K}_{\mathrm{a}} \mathrm{Ti}-\mathrm{K}_{\beta}$ doublet and the Ba- $\mathrm{L}_{a}$ and $B a-L_{\beta}$ lines almost fully overlap.) Since the highly resistant barium sulphate (baryte or permanent white) is very frequently used as substrate, the presence of $\mathrm{Ba}$ is not a surprise, at all. In conclusion, any judgement classifying The third cedar as a forgery should not refer to the presence of titanium white, it should contain other arguments.

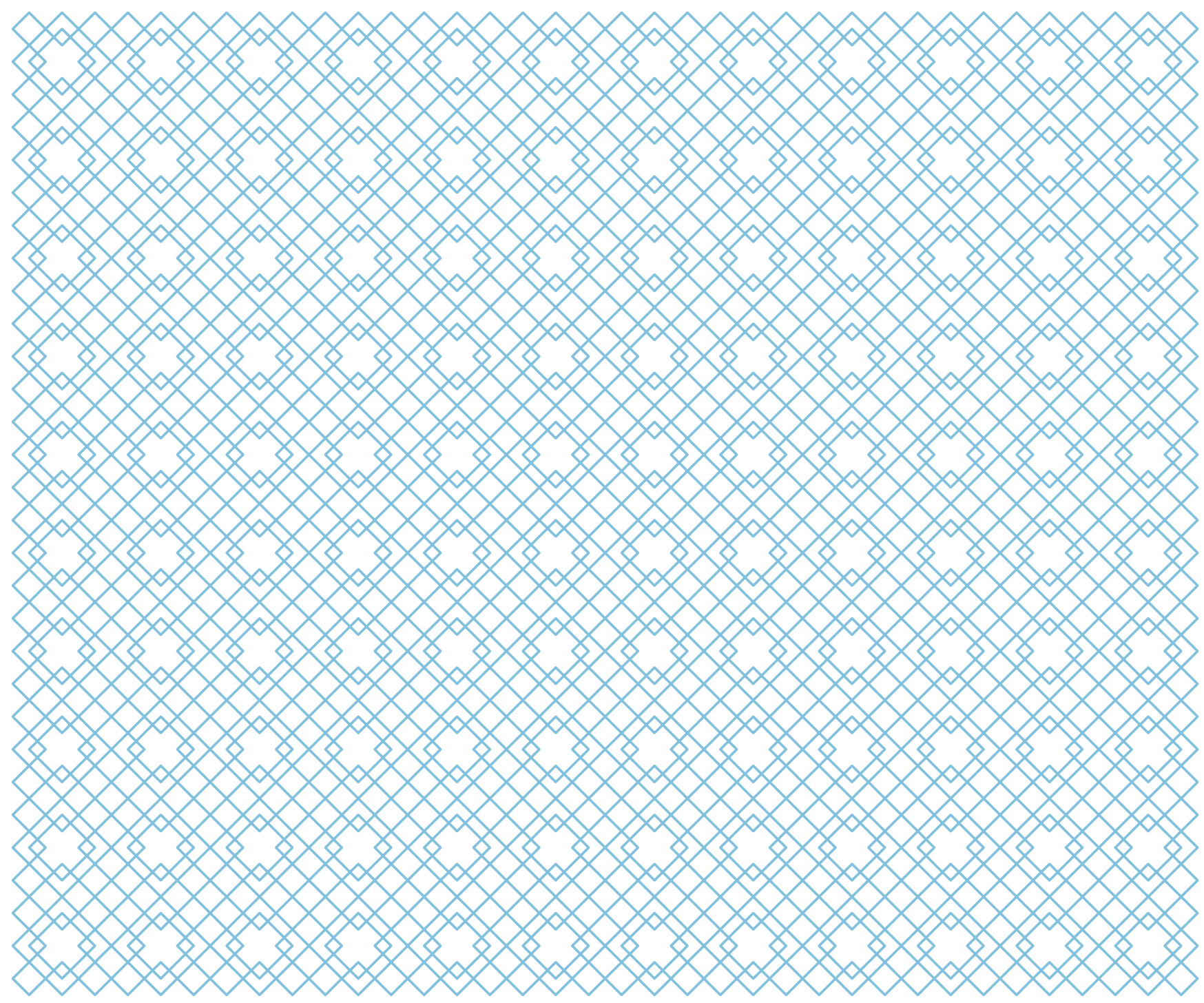




\section{PRESERVATION OF CULTURAL HERITAGE}

lonising radiation is increasingly used in a wide range of industrial and scientific processes, including the sterilisation of medical devices, food irradiation and polymer cross-linking. For more than 40 years industrial irradiation facilities have used gamma rays or electron beams for these purposes. In this chapter we explore applications of ionising radiation in the preservation of cultural artefacts.

\subsection{BASIC PRINCIPLES}

High energy photons or electrons can break molecular bonds, initiating further chemical reactions. The induced scission of both strands in a cell's DNA double helix is the main cause for the radiation biocide effect and the basis of applications related to "killing" unwanted pests (bacteria, fungi or insects). At the same time, care needs to be taken to prevent any unwanted degradation of the irradiated material. The desired effect is achieved by exposing items for a predetermined time in an intense radiation field.

Two irradiation processes have been used in the preservation of cultural artefacts [177-179]. In the first process, radiation treatment is used for the disinfection of insects and moulds in materials.

In addition, resins may be used to impregnate the material and consolidate its structure. Here the role of irradiation is to polymerise the resin. This second technique is especially useful for wooden artefacts. It halts further decay, but does this at the expense of changing the material's structure and properties, including mass and colour. It is irreversible.

Both techniques were initially developed using $y$-radiation. See Figure 6.1. The first experiments using

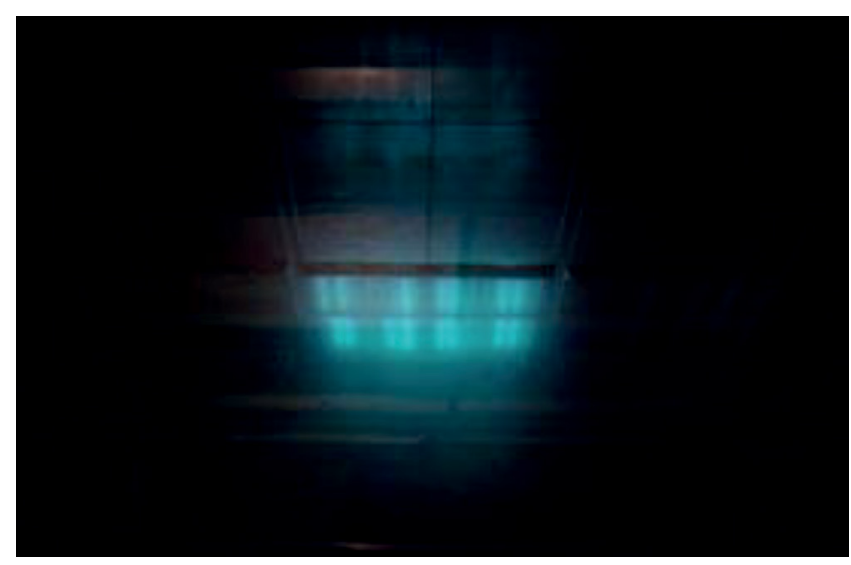

Figure 6.2: High activity ${ }^{60} \mathrm{Co}$ sources (about $500 \mathrm{kCi}$ in 2014) stored under water at the IFIN-HH (National Institute for Nuclear Physics and Engineering "Horia Hulubei", Bucharest, Romania) $y$ irradiation facility. Blue Cerenkov radiation is produced by the high energy $\beta$ particles from the ${ }^{60} \mathrm{Co}$ sources that pass through the water.

$Y$-radiation to preserve cultural artefacts were carried out more than 50 years ago [180].

Even now, $y$-radiation is the most used type of ionising radiation for cultural heritage applications. However, irradiation now also includes accelerator-produced electron beams and $\mathrm{X}$-rays. To avoid disrupting atomic nuclei in the irradiated material, electron beam energies are limited to a maximum of $10 \mathrm{MeV}$ and generated $\mathrm{X}$-rays to a maximum of $5 \mathrm{MeV}$. $y$-rays obtained from radioisotopes generally do not have high enough energies to initiate nuclear reactions.

$\gamma$-emission generally follows spontaneous $\beta$-decay of unstable nuclides. One or more photons with characteristic energies are produced in each decay event. The most widespread y source in current use is ${ }^{60} \mathrm{Co}$ (see Figure 6.2). This artificial isotope is produced on a large scale by activation of ${ }^{59} \mathrm{Co}$ in nuclear reactors. For example, CANDU reactors can produce ${ }^{60} \mathrm{Co}$ in parallel with energy production.
Figure 6.1: Spectrum of electromagnetic radiation. $\mathrm{X}$-rays and $\mathrm{Y}$-rays are ionising radiation, while radio waves, infra-red (IR), visible (VIS) and ultra-violet (UV) radiation do not have enough energy for radiation treatments.

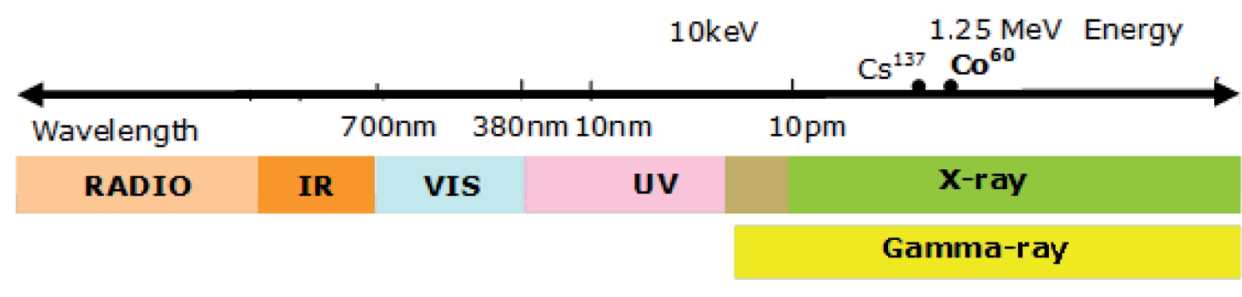




\subsection{INSTRUMENTATION OF NUCLEAR PRESERVATION METHODS}

There is considerable diversity in the design of $y$ irradiation facilities [179] but two characteristics are common: a system for controlling the exposure of irradiated items to the radiation source and shielding to protect humans and the environment from the effects of the high radiation field.

The control of the exposure time is usually achieved by temporarily shielding the radiation source in a lead or concrete containment (dry storage) or using a water pool (wet storage).

The irradiation is carried out in a dedicated room shielded by concrete walls see Figure 6.3. Small research irradiators have their irradiation chambers (cells) shielded by lead but their small size generally makes them unsuitable for the study of cultural artefacts. Industrial scale irradiators tend to have conveyor systems for transporting the goods in and out of a special irradiation room. Some cultural heritage items (paper, textiles, and books) can be loaded in standard boxes increasing the efficiency of the procedure see Figure 6.3.

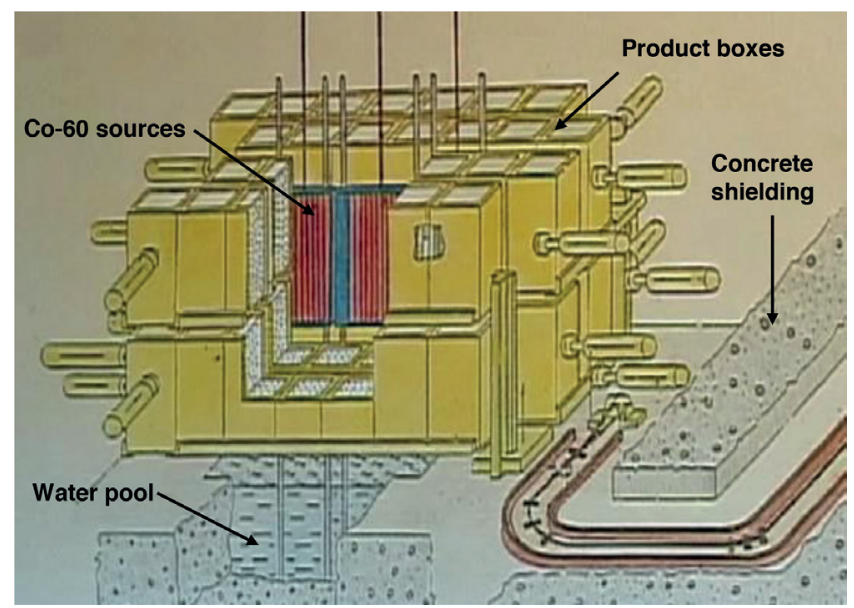

Figure 6.3: Outline of the IFIN-HH ${ }^{60} \mathrm{Co}$ irradiation facility. The concrete shielding is designed for a maximum ${ }^{60} \mathrm{Co}$ intensity of 2 million Ci. Up to $10 \mathrm{~m}^{3}$ of material can be simultaneously irradiated and an extra space is available between the source and the walls (up to $8 \mathrm{~m}^{3}$ ) which allow the irradiation of large items (e.g. large wooden icons or furniture).

For the irradiation consolidation technique some supplementary equipment is required: vacuum/ pressure chambers or impregnation baths are needed to introduce the consolidation matrix into the material being conserved prior to its irradiation [178].

\subsection{APPLICATIONS OF NUCLEAR PRESERVATION METHODS}

\subsubsection{RADIATION DISINFECTION}

Radiation resistance is normally characterised by one of two measures [179]: the so-called $\mathrm{LD}_{50}$ lethal dose (the dose equivalent which kills $50 \%$ of a certain population) or $\mathrm{D}_{10}$ (the absorbed dose which produces a $90 \%$ decrease in a population). Whereas the $L D_{50}$ lethal dose is about 10 Gy for mammals and $100 \mathrm{~Gy}$ for insects, the $\mathrm{D}_{10}$ dose for fungi and bacteria can be $1000 \mathrm{~Gy}$ or more.

Treatment by gamma irradiation: From shortly after its discovery, ionising radiation was observed to produce irreversible damage to living cells. The interaction of radiation modifies molecular structures and bio-chemical processes. Radiation resistance is large for simple organisms (bacteria, fungi) but is much lower for more complex organisms.

Strengths: $y$-rays have a high penetration into artefacts, assuring a strong biocide effect, but do not damage artefact components. The process is fast and ideal for emergency situations.

Weaknesses: The cost of $\mathrm{y}$-irradiation facilities are high, but these can be shared with medical and food irradiation. Stringent radiation protection procedures are necessary. Artefacts have to be transported to the facility.

The biocide effect of ionising radiation was first used for sterilisation of medical equipment in order to obtain materials free of any living micro-organisms. Later it was used in food irradiation to reduce populations of microorganisms or to eradicate insect pests.

These two uses led to major technological developments over the past 50-60 years and produced a large body of data on the radiation resistance of micro-organisms and insects. To a good approximation, the survival curve for a given micro-organism is observed to follow an exponential decay (See Figure 6.4). This allows the disinfection rate due to ionising radiation to be predicted.

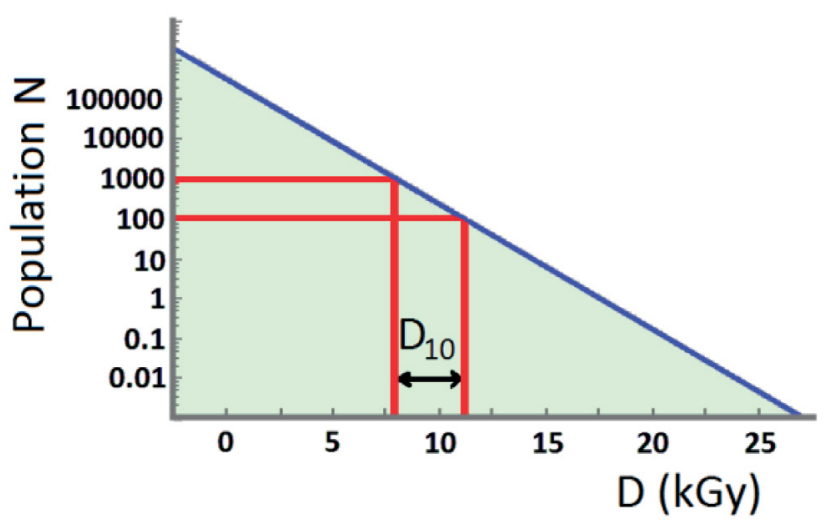

Figure 6.4: Schematic survival curve showing an exponential relation between the surviving population of a micro-organism population $\mathrm{N}$ and its absorbed radiation dose $\mathrm{D}$. $\mathrm{D}_{10}$ is the absorbed dose which produces a $90 \%$ decrease in a population. 
Many cultural heritage items are threatened by biological attacks. The organic constituents (cellulose, collagen or proteins) are sources of food for microorganisms and insects. Improper storage conditions lead to active attacks even in museums, archives or libraries. Such attacks are difficult to stop or eradicate because of the large quantities of items involved. Conservators and restorers can easily treat individual items but the mass treatment of collections is problematic.

Methods involving fumigation, liquid chemicals or anoxic atmospheres have their own particular advantages and disadvantages in terms of efficacy and efficiency which we will not detail. Rather we will focus on gamma radiation treatment, in which the high penetration of ionising radiation assures the required biocide effect.

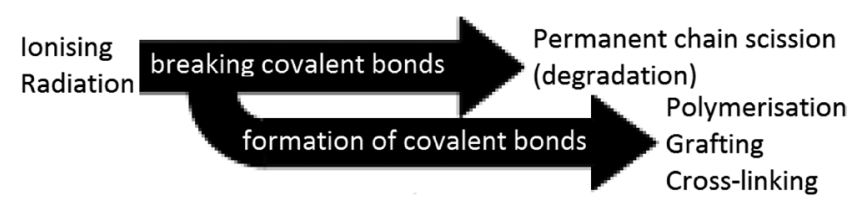

Figure 6.5: The contrasting effects of ionising radiation on macro-molecules.

\section{Wood}

Wooden artefacts are commonly affected by woodworm or wood-boring beetle infestations. The radiation resistance of insects is relatively low and these pests are easily eliminated by irradiation, regardless of their stage of development (larvae, eggs). Fungi are inactivated at higher irradiation doses, which are well tolerated by wood. Despite the fact that cellulose is known to be degraded by irradiation, the lignin-cellulose assembly shows no changes when subjected to a large dose. If wood is painted, there is a question as to the extent to which the pigments or varnish of the artwork are affected by radiation. In the literature there are reports on the dose responses of more than 30 pigments and varnishes. It is generally accepted that a dose of $10 \mathrm{kGy}$ will not induce painting degradation, while insects and most fungi are killed $[178,181]$.

The consolidation of wooden samples by impregnation with resins, hardened by radiation polymerisation, is a specialist application that merits separate discussion. In this case, the disinfection is implicit and an important improvement in the mechanical strength of highly degraded wood may be obtained.

\section{PAPER}

The major threat to paper artefacts is from fungi. Increased humidity can easily activate fungal growth, which can spread rapidly. Some bacteria also affect paper and woodworms can damage collections of books with wooden covers. Other insects do not digest cellulose but are attracted by binding materials and contribute to the disintegration of books creating a premise for further micro-biological attack.

Paper is made from cellulose fibres but with no, or low, lignin content. Consequently degradation due to irradiation is an issue and solutions are difficult. At low irradiation doses the degree of cellulose polymerisation is reduced. See Figure 6.5. However, this is not reflected in the macroscopic properties of the paper. The determination of the irradiation dose is sensitive and should take into consideration the severity of the biological attack and the radiation resistance of the contaminants. A number of reports show no changes to paper properties (mechanical strength, colour, $\mathrm{pH}$, water sorption, etc.) for doses in the range of 5 to $10 \mathrm{kGy}$ [181-183].

\section{Textiles, leather and parchment}

Textiles can be equally affected by insects, fungi or bacteria. Micro-organisms (fungi) are the main threat for historical leather. Sometimes, insects (woodworms in the case of old book covers) are also destructive. Due to reduced water retention, parchment is more resistant to pest attacks but is generally fragile because of its age [184]. The lack of information on the irradiation effects on textile, leather or parchment artefacts can be attributed to the smaller quantities of such items. The selection of the irradiation dose should take into consideration each of the materials (cotton, wool, silk, leather, etc) and the extent to which the artefacts have already been degraded by biological attack [179].

\subsubsection{IRRADIATION CONSOLIDATION OF WOODEN ARTEFACTS}

Consolidation of wooden artefacts by impregnation with synthetic polymers, radiation polymerisation and/ or cross-linking is a field which involves both radiation physics and radiation chemistry. In the case of disinfection the only concern is to not have unwanted degradation of the materials; for consolidation a careful control of the chemical processes induced by radiation is essential. Dose, dose-rate, and concentration of reagents are all parameters which need to be controlled. Care also needs to be taken as exothermic reactions can lead to irreversible heat damage to artefacts.

Research on this topic started worldwide in the 1960s but the field was pioneered and first applied on a large scale in ARC-NUCLEART (Atelier Regional de Conservation), Grenoble, France [185].

Starting in 1970, NUCLEART have a long history of achievements for both radiation disinfection and radiation consolidation [186-188]: consolidation of the $19^{\text {th }} \mathrm{C}$. mosaic parquetry (flooring) of ancient Grenoble city hall (1970); disinfection of the famous Pharao Ramses II mummy from the Cairo Egyptian Museum (1977); disinfection of the baby mammoth Kroma, found in permafrost soil in Northern Siberia (2010). The works 
developed currently at NUCLEART include consolidation and restoration of a large number of religious items (e.g. statues) and very large wooden objects (e.g. furniture pieces).

\begin{abstract}
APPLICATION
The principle of consolidation is in many ways similar to methods for producing composites. A close contact between the material and the consolidation matrix is necessary. In case of cultural heritage artefacts, air or water is removed from the pores and micro-pores of the material and a liquid matrix is introduced. The procedure can be applied to various materials, but the best results are obtained with degraded wood. The weight of the object is increased by $50-100 \%$. $A$ range of different consolidant materials have been tried (monomers, pre-polymers) but the best results have been obtained using radiopolymerisation of polyester resins.
\end{abstract}

A special technique was developed at NUCLEART for waterlogged wood consolidation (e.g. parts of ancient Viking shipwrecks). Consolidation gives a new lease of life for highly degraded wooden artefacts and, in some cases, is the only practical preservation method (e.g. for waterlogged wood) $[179,187]$.

\subsubsection{GAMMA RAY TREATMENTS AT IRASM IFIN-HH}

$\mathrm{Y}$-irradiation treatments to preserve cultural heritage form an important project at the IRASM - Radiation Processing Centre of IFIN-HH. Experiments started 20 years ago, and large quantities of important cultural artefacts (wood, polychrome wood, paper, textiles, leather and parchment items) have been treated since then $[179,189]$.

One remarkable achievement was the irradiation of the iconostases from two Romanian historical churches [189]. See Figures 6.6 and 6.7. The treatment was reported as a success story by the International Agency for Atomic Energy (IAEA) in 2012. An economic study revealed that restoration of the iconostases costs only $10 \%$ of the price of producing new ones. A classical (chemical) disinfection treatment would have required years of work; whereas using radiation treatment the iconostases were fully restored in several months. For transport and irradiation, the large iconostases $\left(6 \times 4 \mathrm{~m}^{2}\right)$ were dismantled into pieces up to $2.5 \mathrm{~m}$ in size. Several collections, containing more than 400 icons, with sizes from $30 \times 40 \mathrm{~cm}^{2}$ to $150 \times 200 \mathrm{~cm}^{2}$ were also treated.

An extremely complex irradiation treatment was performed in the case of the "Theodor Aman" Museum, of the Bucharest City Museums network [179]. The building,

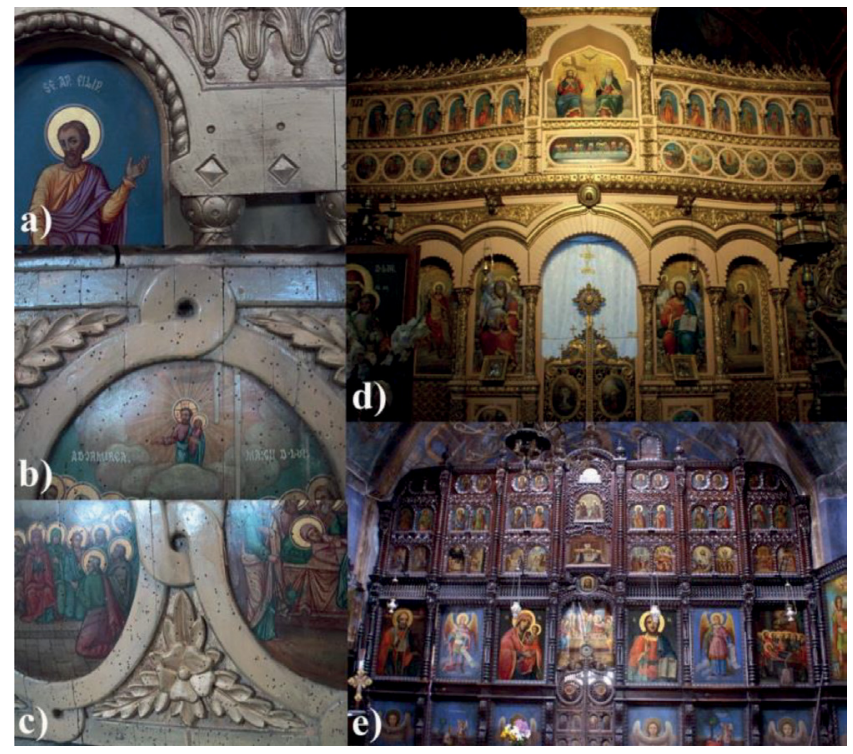

Figure 6.6: Iconostases from Izvoarele churches in Dambovitza county, Southern Romania: a), b) and c) details of parts of the iconostases, showing marks (holes) due to woodworm attack; d) and e) iconostases of the two churches after their complete restoration.

a temple of art in neo-classical and Renaissance style, suffered severe damage due to lack of care in recent years. The damaged roof produced advanced degradation of both the building structure and the collections it contained. When funding became available for a complete refurbishment of the building, a disinfection of its complete inventory was necessary.

The decision was taken to use radiation treatment. All the items from the Memorial museum (furniture, flooring, doors, wainscot panelling, wallpaper, tools and the personal items of the painter) were treated to remove woodworm and fungi. Today the museum is open to the public.

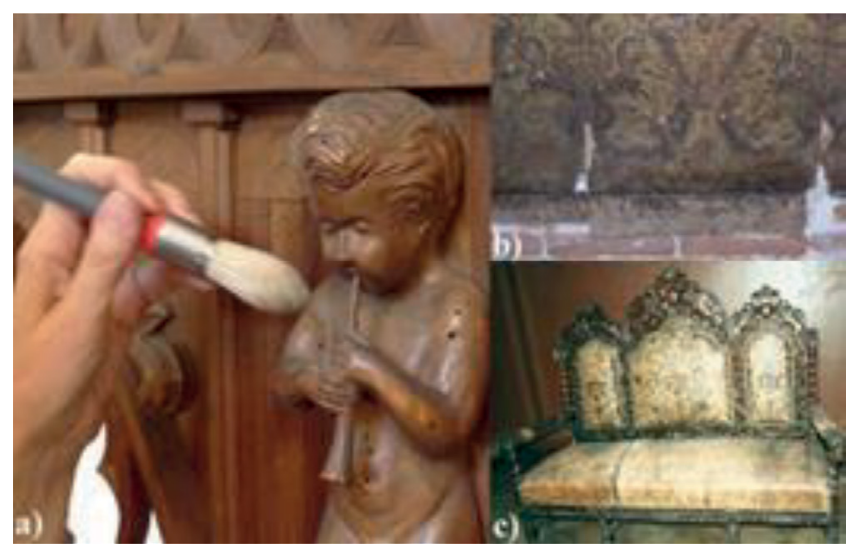

Figure 6.7: Items from the Theodor Aman Museum: a) detail of a cabinet with woodworms marks; b) and c) wallpaper and sofa with marks due to fungal attack.

Romanian painter (1831-1891) 


\subsubsection{IRRADIATION CONSOLIDATION OF WOODEN ARTEFACTS AT IRASM IFIN-HH}

Since 2012 co-operation with NUCLEART, Grenoble, France, has helped IRASM IFIN-HH to introduce irradiation consolidation methods to Romania. The first work was performed using test equipment designed for the consolidation of small items. See Figure 6.8.

Irradiation consolidation is an irreversible treatment which is sometimes controversial because it changes the original material. However, it gives new life to highly degraded items which are impossible to save using other preservation methods.

A first test of this technique was performed on a traditional wooden object: an old butter churn. Before consolidation, this churn could not support its own weight. After irradiation consolidation it gained more than $50 \%$ in weight and can now stand in an upright position. Consolidation was performed with a polyester-styrene mixture (NUCLEART method). After irradiation the styrene

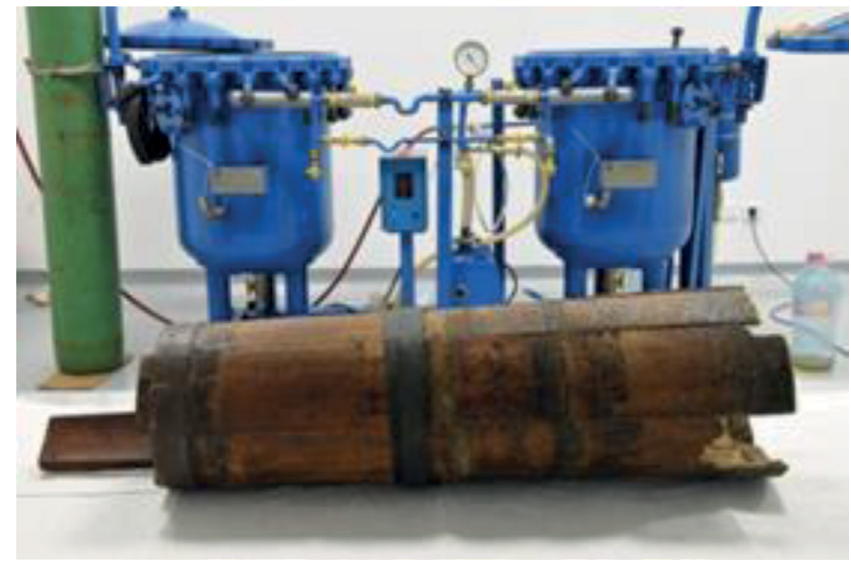

Figure 6.8: Test consolidation facility at IRASM IFIN-HH: 100 liter vacuum/pressure impregnation equipment (back); wooden butter churn degraded by woodworm atack and consolidated by radio-polymerisation (front).

formed bridges between the polyester chains and created a tri-dimensional molecular structure [188].

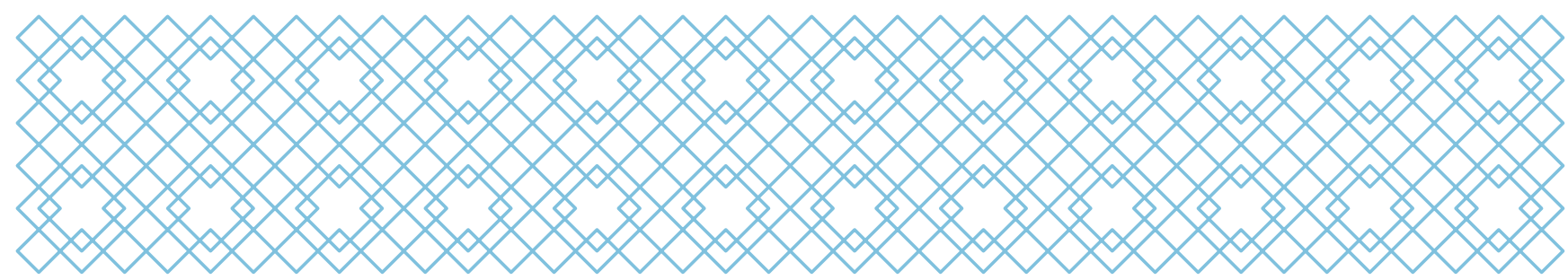

\section{CONCLUSION}

T he application of atomic and nuclear techniques to the study of archaeological objects gives the historian or archaeologist material information that can help them to understand life during ancient times. This knowledge is necessary to test the authenticity and provenance of artefacts and to prepare and carry out necessary restorations. All these objectives are common to a very large community of people working in the field of archaeometry, i.e. the "application of science to art and archaeology". For all these research activities a multi-disciplinary approach is essential, bringing together physicists, chemists, archaeologists, numismatists, historians, geologists and conservators from different laboratories, institutions and museums.

This topical paper, brought to you by the Nuclear Physics Division of the European Physics Society, provides a useful opportunity to show the public, and also the professional community, just how broad and important the field of application of nuclear techniques has become in the study of cultural heritage, its characterisation and preservation.

This publication reports the very fruitful collaboration of many scientists from different fields who are all focused on the same goal - to enlarge and deepen our knowledge about our cultural heritage and to keep it safe for future generations. 


\section{APPENDIX A: EUROPEAN FACILITIES USING NUCLEAR TECHNIQUES TO STUDY CULTURAL HERITAGE}

The map below shows laboratories and centres with facilites relevant to nuclear physics studies of Cultural Heritage objects. These are grouped into four categories:

$\square$ Ion Beam Analysis Facilities in Europe

$\square$ European Accelerator Mass Spectrometry Facilities

$\square$ European Neutron Sources

$\square$ Other European Centres, Facilities and Laboratories

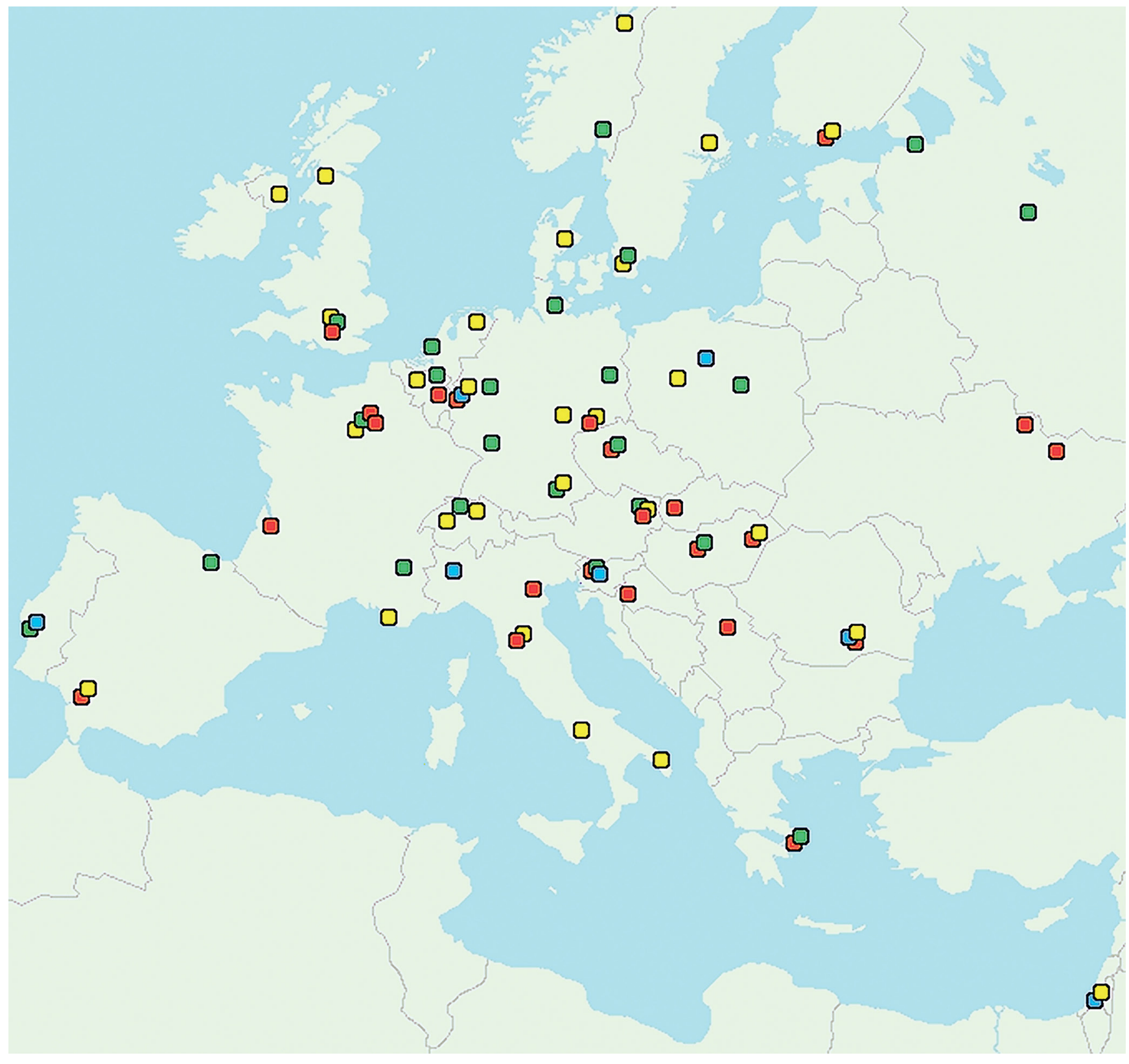




\section{ION BEAM ANALYSIS FACILITIES IN EUROPE}

Austria, NSIL Nuclear Science and Instrumentation Laboratory, IAEA, Seibersdorf, https://www.seibersdorf-laboratories.at/en/home

$\square$ Belgium, Centre Européen d'Archéométrie (CEA) \& Institut de Physique Nucléair, University of Liege, http://www.cearcheo.ulg.ac.be/Accueil.html

$\square$ Belgium, ALTAïS Accélérateur Linéaire Tandetron pour l'Analyse et l'Implantation des Solides, Namur http://www.unamur.be/sciences/physique/larn/

- Croatia, Laboratory for Ion Beam Interactions, Ruđer Bošković Institute, CEMS Centre of Excellence for Advanced Materials and Sensing Devices, Zagreb, http://cems.irb.hr/en/

$\square$ Czech Republic, CANAM Centre for Accelerators and Nuclear Analytical Methods, Nuclear Physics Institute, ASCR Academy of Sciences of the Czech Repullic, Řež, http://canam.ujf.cas.cz/

$\square$ Finland, University of Helsinki, Ion beam analysis laboratory, Helsinki, https://tuhat.halvi.helsinki.fi/portal/en/equipment/ion-beam-analysis-1(2c267889-4d75-446f-95b3-c1384de3e9c4).html

- France, AGLAE, Accélérateur Grand Louvre d'Analyses Elémentaires, Paris http://c2rmf.fr/analyser/un-laboratoire-de-haute-technologie-pour-les-collections-des-musees/aglae

口 France, CENBG Centre d'Études Nucléaires de Bordeaux Gradignan, http://www.cenbg.in2p3.fr/

$\square$ France, SAFIR Système d'Analyse par Faisceaux d'Ions Rapides, Institut des NanoSciences de Paris, http://www.insp.jussieu.fr/Systeme-d-Analyse-par-Faisceaux-d.html

$\square$ Germany, HZDR Helmholtz Zentrum Dresden-Rossendorf, Ion Beam Analysis Group, Dresden, http://www.hzdr.de/db/Cms?pOid=35575\&pNid=2744

$\square$ Greece, NCSR National Centre for Scientific Research DEMOKRITOS, Athens, http://www.demokritos.gr/

$\square$ Italy, INFN LABEC, Florence, http://labec.fi.infn.it/

— Italy, Laboratori Nazionali di Legnaro, AN2000 accelerator, INFN Legnaro, http://www.Inl.infn.it/index.php/en/ accelerators-3/an-2000

- Hungary, MTA Atomki, Laboratory of Ion Beam Applications, Institute for Nuclear Research, Debrecen, http://w3.atomki.hu/index_en.html

$\square$ Hungary, MTA Wigner FK, Department for Materials Science by Nuclear Methods, Wigner Research Centre for Physics, Budapest, http://wigner.mta.hu/en

$\square$ Romania, Horia Hulubei National Institute for Nuclear Physics and Engineering, Bucharest-Magurele, http://www.nipne.ro/

- Serbia, FAMA Facility for Modification and Analysis of Materials with Ion Beams, Vinča Institute of Nuclear Sciences, Belgrade, http://www.vin.bg.ac.rs/010/index.html

$\square$ Slovak Republic, Advanced Technologies Research Institute, Trnava, http://www.mtf.stuba.sk/english/institutes/advanced-technologies-research-institute/

口 Slovenia, MIC Microanalytical Centre, Jožef Stefan Institute (JSI), Ljubljana, http://www.rcp.ijs.si/mic/

$\square$ Spain, Centro Nacional de Aceleradores, CNA, is a joint centre of Universidad de Sevilla, Junta de Andalucía and CSIC, Seville, http://acdc.sav.us.es/cna/

口 United Kingdom, Ion Beam Centre, University of Surrey, Guildford, http://www.surrey.ac.uk/ati/ibc/

$\square$ Ukraine, ISSPMT Institute of Solid State Physics, Materials Science and Technologies, National Academy of Sciences of Ukraine, Kharkov, http://www.kipt.kharkov.ua/kipt_sites/isspmst/main_site/ENG34Main.html

- Ukraine, Institute of Applied Physics, National Academy of Sciences of Ukraine, SUMY, http://iap.sumy.org/viewexpbase/en/?id=15

\section{EUROPEAN NEUTRON SOURCES}

Austria, TRIGA Mark II Reactor, TU Vienna, Vienna, http://ati.tuwien.ac.at/reactor/EN/

$\square$ Belgium, IRMM Institute for Reference Materials and Measurements, Geel, https://ec.europa.eu/jrc/en/institutes/irmm

$\square$ Czech Republic, Department of Neutron Physics, Nuclear Physics Institute, ASCR, Academy of Sciences of the Czech Republic, Řež, http://neutron.ujf.cas.cz/

$\square$ Europe, Central European Research Infrastructure Consortium (CERIC-ERIC), http://www.ceric-eric.eu/

$\square$ France, Institut Laue-Langevin (ILL), Neutrons for Science, https://www.ill.eu/

- France, Laboratoire Léon Brillouin (LLB), Gif-sur-Yvette, http://www-llb.cea.fr/en/

$\square$ Germany, Forschungs-Neutronenquelle Heinz Maier-Leibnitz (FRM II), Garching, https://www.frm2.tum.de/en/home/

$\square$ Germany, GEMS German Engineering Materials Science Centre, Helmholtz-Zentrum Geesthacht, http://www.hzg.de/index.php.de 
Germany, Helmholtz-Zentrum Berlin für Materialien und Energie (HZB), Berlin, https://www.helmholtz-berlin.de/index_en.html

口 Germany, Jülich Centre for Neutron Science, Jülich, http://www.fz-juelich.de/jcns/EN/Home/home_node.html

$\square$ Germany, TRIGA Mark II reactor, Johannes Gutenberg University, Mainz, http://www.kernchemie.uni-mainz.de/eng/234.php

$\square$ Greece, NCSR National Centre for Scientific Research DEMOKRITOS, http://www.demokritos.gr/

- Hungary, Budapest Neutron Centre (BNC), Budapest, http://www.bnc.hu/

T The Netherlands, RID Reactor Institute Delft, Delft, http://www.rid.tudelft.nl/en

$\square$ Norway, IFE Institute for Energy Technology, Kjeller, http://www.ife.no/en

$\square$ Poland, MARIA National Centre for Nuclear Research, Otwock-Świerk, http://www.ncbj.gov.pl/en/maria

口 Portugal, Centro de Ciências e Tecnologias Nucleares (C2TN), Instituto Superior Técnico (IST), Lisbon, http://c2tn.tecnico.ulisboa.pt/en

Russia, Joint Institute for Nuclear Research (JINR), Dubna, http://wwwold.jinr.ru/

口 Russia, PNPI Petersburg Nuclear Physics Institute, Gatchina, St. Petersburg, http://www.pnpi.spb.ru/

$\square$ Slovenia, TRIGA Reactor Infrastructure Centre, Ljubljana, http://www.rcp.ijs.si/ric/reactor-a.htm

$\square$ Spain, ESS Bilbao, Bilbao http://www.essbilbao.org/index.php/en/

$\square$ Sweden, MAX IV and European Spallation Source (ESS), Lund, http://www.lunduniversity.lu.se/research/max-iv-and-ess

$\square$ Switzerland, SINQ: The Swiss Spallation Neutron Source, Paul Scherrer Institute, Villigen, https://www.psi.ch/sinq/

U United Kingdom, ISIS Neutron and Muon Source, Didcot, http://www.isis.stfc.ac.uk/

\section{EUROPEAN ACCELERATOR MASS SPECTROMETRY FACILITIES}

Austria, VERA Vienna Environmental Research Accelerator, Universität Wien, Vienna, https://isotopenforschung.univie.ac.at/en/vera/

$\square$ Belgium, Radiocarbon Dating Laboratory, RICH Royal Institute for Cultural Heritage, Brussels, http://www.kikirpa.be/EN/52/353/Datation+C14.htm

$\square$ Denmark AMS Department. of Physics and Astronomy, Aarhus University, Aarhus, http://phys.au.dk/en/research/research-areas/accelerator-mass-spectroscopy/

$\square$ Finland lon beam analysis laboratory, Department of Physics, University of Helsinki, Helsinki, http://www.physics.helsinki.fi/tutkimus/mat/english/research/ionbeam

- France, ARTEMIS, Pelletron CEA, Saclay, Gif-sur-Yvette, http://www2.cnrs.fr/en/291.htm

$\square$ France, CEREGE - Centre Européen de Recherche et d'Enseignement en Géosciences de l'Environment, Marseille, https://www.cerege.fr/

$\square$ Germany, Max Planck Institute for Biogeochemistry, University of Jena, Jena, http://www.bgc-jena.mpg.de/

$\square$ Germany, GAMS Accelerator Mass Spectrometry Group, Maier-Leibnitz-Laboratory, Garching, http://www.gams.ph.tum.de/

$\square$ Germany, HZDR Helmholtz Zentrum Dresden Rossendorf, Ion Beam Centre, Dresden, http://www.hzdr.de/db/Cms?pOid=27781\&pNid=2791

$\square$ Germany, Centre for Accelerator Mass Spectrometry, University of Cologne, Cologne, http://www.cologneams.uni-koeln.de

$\square$ Hungary, Hertelendi Laboratory of Environmental Studies, Debrecen, http://w3.atomki.hu/hekal/

$\square$ Israel, Max Planck Weizmann Joint Center in the Field of Integrative Archaeology and Anthropology, Weizmann Institute, Rehovot, http://www.weizmann.ac.il/pages/center-of-integrative-archaeology-and-anthropology

$\square$ Italy, CEDAD Centro di Datazione e Diagnostica, Lecce Tandetron Laboratory, Lecce, http://cedad.unisalento.it/en/tandetron.php

$\square$ Italy, CIRCE Center for Isotopic Research on the Cultural and Environmental heritage, seconda universita degli studi di Napoli, Naples, http://www.matfis.unina2.it/dipartimento-205/laboratori/CIRCE

$\square$ Italy, INFN LABEC, Florence http://labec.fi.infn.it/

$\square$ The Netherlands, Radio Carbon AMS, University of Groningen, Groningen, http://www.rug.nl/research/isotope-research/projects/radiocarbon/radiocarbonams

$\square$ Norway, NTNU The National Laboratory of Age Determination, Trondheim, https://www.ntnu.edu/museum/archaeometry

$\square$ Poland, Poznan Radiocarbon Laboratory, Poznan, http://radiocarbon.pl/

$\square$ Romania, Horia Hulubei National Institute for Nuclear Physics and Engineering, Bucharest-Magurele, http://www.nipne.ro/facilities/facilities/tandetron1mv.php 
Spain, Centro Nacional de Aceleradores, CNA, is a joint centre of Universidad de Sevilla, Junta de Andalucía and CSIC, Seville, http://acdc.sav.us.es/cna/

$\square$ Sweden, Radiocarbon Dating Laboratory, Lund University, Lund, http://www.geol.lu.se/c14/en/

$\square$ Sweden, AMS Department of Physics and Astronomy, University of Uppsala, Uppsala, http://www.physics.uu.se/research/applied-nuclear-physics/groups/ion-physics

$\square$ Switzerland, LARA AMS Laboratory, University of Bern, Bern, http://www.14c.unibe.ch/

$\square$ Switzerland, LIP Laboratory for Ion Beam Physics, EHT Zürich, http://www.ipp.phys.ethz.ch/research/ion-beam-physics.html

$\square$ United Kingdom, 14Chrono Centre for Climate, the Environment, and Chronology, Queen's University, Belfast http://www.chrono.qub.ac.uk/

$\square$ United Kingdom, Oxford Radiocarbon Accelerator Unit, Oxford, https://c14.arch.ox.ac.uk/embed.php?File=oxcal.html

$\square$ United Kingdom, SUERC Scottish Universities Environmental Research Centre, East Kilbride, http://www.gla.ac.uk/suerc/

\section{OTHER EUROPEAN CENTRES, FACILITIES AND LABORATORIES}

\section{Magnetic Resonance Centres}

$\square$ Germany, MARC Magnetic Resonance Center, RWTH Aachen University, Aachen www.marc.rwth-aachen.de

$\square$ Slovenia, NMR Centre, Ljubliana http://www.nmr.ki.si/

\section{Gamma Sources}

$\square$ Romania, Extreme Light Infrastructure - Nuclear Physics (ELI-NP), Horia Hulubei National Institute for R\&D in Physics and Nuclear Engineering, Bucharest-Magurele http://www.eli-np.ro/

\section{Luminescence Research}

$\square$ Israel, Geological Survey of Israel, Jerusalem, Luminescence Dating Laboratory, http://www.gsi.gov.il/

$\square$ Poland, Institute of Physics, Faculty of Physics, Astronomy and Informatics, Nicolaus Copernicus University, Toruń, https://www.umk.pl/en/

$\square$ Portugal, Centro de Ciências e Tecnologias Nucleares (C2TN), Instituto Superior Técnico (IST), Lisbon, http://c2tn.tecnico.ulisboa.pt/en

\section{Surface Research}

$\square$ Italy, NIS centre for nanostructured interfaces and surfaces, Universita degli Studi di Torino http://www.nis.unito.it/index.html

\section{X-ray applications}

$\square$ Romania, Accent Pro 2000 S.R.L. (AP2K) Research \& Development Company http://www.accent.ro/

\section{European Research Consortia}

$\square$ Integrated Platform for the European Research Infrastructure ON Culture Heritage (IPERION CH), http://www.iperionch.eu/

$\square$ Central European Research Infrastructure Consortium (CERIC), http://www.ceric-eric.eu/

$\square$ Neutron Scattering and Muon Spectroscopy (NMI3), http://nmi3.eu/

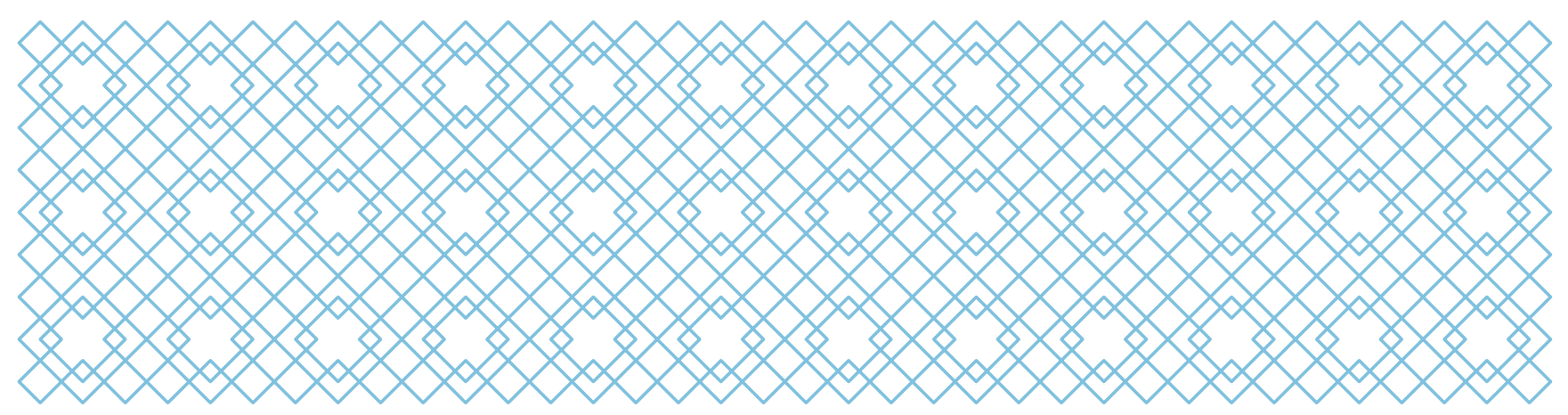




\section{APPENDIX B: GLOSSARY OF TERMS}

(a,b) Nuclear reaction: a target is hit by particle a and particle $b$ is emitted.

\section{AMS Accelerator Mass Spectrometry}

Antiquity The period of human history and prehistory prior to the Middle Ages ( $6^{\text {th }}-15^{\text {th }}$ centuries).

Anti-neutrino A very light, neutral anti-particle emitted in $\boldsymbol{\beta}$-decay.

Alpha (a) particles A heavy, positive, doubly charged particle emitted from some unstable heavy nuclei.

Archaeometallurgy The study of human use of metals in antiquity.

Beta $(\boldsymbol{\beta})$ particles A light, singly charged particle, emitted in $\beta^{-}$-decay. The negatively charged $\beta^{-}$particle has the same properties as an electron. Its $\beta^{+}$anti-particle is positively charged and is emitted in $\beta^{+}$-decays.

Bomb peak A peak in ${ }^{14} \mathrm{C}$ concentration which occurs in biological cells created after the 1950s. It is due to the effects of atmospheric nuclear tests and is relevant to the interpretation of radiocarbon dating measurements.

${ }^{14} \mathrm{C} \quad \beta$-unstable Carbon isotope with 6 protons and 8 neutrons.

Charged particles Particles such as protons and electrons which carry electric charge.

CT Computed tomography: a 2D x-ray or $\mathrm{Y}$-ray image constructed from multiple 1D images taken at different angles.

Concentration The relative abundance of a constituent part or element. Concentration is often presented in units of atomic percent (at.\%).

CPMG sequence Car-Purcell-Meiboom-Gill sequence of radio-frequency pulses commonly used in NMR imaging.

Cross section A measure of the probability, or likelihood, of a reaction taking place

Cultural heritage Artefacts which represent aspects of history of human culture, civilisation and society.

Curation The preservation, cataloguing and safeguarding of historical artefacts.

Deuterium A heavy Hydrogen atom whose atomic nucleus contains one proton plus one neutron.
Dosimetry The measurement of exposure to ionising radiation.

DNA DeoxyriboNucleic Acid. DNA is the building block of biological cells, encoding genetic information.

EDX Energy Dispersive X-ray spectroscopy is an electron-induced $X$-ray technique used for elemental analysis, often used in conjunction with electon microscopy.

Electrons Light, negatively charged particles which are part of each atom, orbiting its positively charged nucleus.

Element A substance which cannot be broken into simpler substances by chemical means. Each element has unique physical and chemical properties and its own place in the periodic table of the elements. An atom is the smallest unit of each element.

Elemental analysis An analysis of which elements which make up an object.

ELI-NP Extreme Light Infrastructure-Nuclear Physics: a very high intensity laser and $\mathbf{y}$-beam facility for Nuclear Physics studies, Bucharest-Magurele, Romania.

ERDA Elastic Recoil Detection Analysis.

$\mathbf{e V}, \mathbf{k e V}, \mathbf{M e V}$ The eV is a unit of energy, specifically the energy an electron acquires when it passes a potential difference of $1 \mathrm{~V}$; keV (1000s of eV) are typical energies of X-rays; $\mathrm{MeV}(1,000,000$ s of $\mathrm{eV})$ are typical energies of $\mathrm{Y}$-rays.

External beam A beam of particles outside a vacuum chamber, often emerging through a thin window.

Y-rays Very high energy electromagnetic radiation in the MeV energy range, produced by high energy bremsstrahlung or transitions between nuclear energy levels.

Ge(Li) Lithium-drifted Germanium solid state detector, used to measure $\gamma$-ray energies.

Half-life The characteristic time taken for the intensity of a radioactive substance to reduce by a factor of two.

Heavy ions Charged atoms from heavy elements

HIERDA Elastic Recoil Detection Analysis using a heavy ion beam.

Homogeneous Having the same value of some quantity

HPGe Hyper-pure Germanium detector, used to measure Y-ray energies. 
IAEA International Atomic Energy Agency

Ion Beams A beam, or flow, of flux of positively, or negatively, charged atoms

IBA Ion Beam Analysis.

IBIL Ion Beam Induced Luminescence.

INAA Instrumental Neutron Activation Analysis.

INFN Italian Nuclear Physics Research Agency: Istituto Nazionale di Fisica Nucleare

Inhomogeneous Having non-constant values of some quantity

In-situ In its customary position, not in a laboratory.

Ionising radiation Radiation which causes ionisation of atoms. Ionising radiation includes ion beams, a-particles, $\beta$-particles, $\gamma$-rays and $X$-rays. Neutron radiation does not directly cause ionisation, but can also lead to the break-up of atoms.

Irradiation Subjecting an object to a dose of radiation.

ISIS Research laboratory near Oxford, UK, with spallation neutron beam facilities.

Isotope An atom with a particular number of neutrons in its nucleus.

LABEC Laboratorio di Tecniche Nucleari per i Beni Culturali, in Florence, Italy.

Luminescence Emission of light from energy stored in excited atomic states.

OSL Optically-stimulated luminescence.

NAA Neutron Activation Analysis.

NDP Neutron Depth Profiling.

Neutrons Heavy neutral particles, found as constiuents of atomic nuclei.

NMR Nuclear Magnetic Resonance.

NMR-MOUSE Nuclear Magnetic Resonance-Mobile Universal Surface Explorer: a small portable NMR measurement system capable of measuring depth profiles.

NRA Nuclear Reaction Analysis.

NRF Nuclear Resonance Fluorescence.

$\boldsymbol{\mu}$-IBIL Micro-beam Ion Beam Induced Luminescence.

$\mathbf{\mu - X R F \quad M i c r o - b e a m ~ X - R a y ~ F l u o r e s c e n c e . ~}$

PGAA or PGNAA Prompt Gamma Activation Analysis.

PIGE Particle-Induced $\gamma$-ray Emission.

PIGME Particle-Induced $\gamma$-ray Emission.

PIXE Particle-Induced X-ray Emission.
PMP Proton Micro Probe.

Protons Heavy positively charged particles, found as constiuents of all atomic nuclei.

Provenance The source or origin of an object or artefact.

Radiocarbon dating Dating of historical objects containing carbon by measuring their ${ }^{14} \mathrm{C}$ concentration.

Raman Spectroscopy Optical technique used to identify molecules.

RBS Rutherford Back-Scattering.

RBS/C RBS-Channelling spectrometry.

REE Rare Earth Element. One of 17 heavy elements which have a low natural abundance. Rare Earth Elements include the complete Lanthanide series of elements

Reactor A vessel in which a controlled nuclear reaction takes place. Reactors are commonly used as intense sources of neutron beams.

RNAA Radiochemical separation of a sample undergoing Neutron Activation Analysis.

Relaxation time The characteristic time taken for magnetic polarisation to decay in NMR studies.

RNRA Resonant Nuclear Reaction Analysis.

SANS Small Angle Neutron Scattering.

SEM-EDX Scanning Electron Microscopy coupled with Energy Dispersive X-ray spectroscopy.

SDD Silicon Drift Detector: a semiconductor detector commonly used to detect $\mathrm{X}$-rays.

SIMP Scanning Ion Micro Probe.

SINQ The Swiss Spallation Neutron Source at the Paul Scherrer Institute, Villigen, Switzertland.

$\mathrm{Si}(\mathrm{Li}) \quad$ Lithium-drifted Silicon solid state detector, used to measure $\mathrm{X}$-ray energies

TL Thermo-Luminescence.

Trace element Elements which occur as a low-concentration constituent or impurity in an object.

TOF Time-of-Flight: a technique used to measure particle energies by the time they take to cover a known distance.

Triptych A three-panel painting or icon.

TOF-ND Time-of-flight neutron diffraction.

X-rays High energy electromagnetic radiation in the keV energy range, produced by electron bremsstrahlung or transitions between atomic energy levels.

XRF X-Ray Fluorescence. 


\section{APPENDIX C: EXPERTISE OF AUTHORS}

\section{Bogdan Constantinescu}

Horia Hulubei National Institute for Nuclear Physics and Engineering, Bucharest, Romania. XRF and micro-PIXE analysis of archaeological artefacts and geological samples.

\section{Bernhard Blümich}

Professor of Macromolecular Chemistry at the RWTH Aachen University, Germany. Specialist in nuclear magnetic resonance instrumentation, methods, and applications to materials and processes.

\section{Dimiter L. Balabanski}

Extreme Light Infrastructure - Nuclear Physics, Horia Hulubei National Institute for R\&D in Physics and Nuclear Engineering, Bucharest-Magurele, Romania. Senior researcher, Head of research on nuclear physics and applications with high-brilliance gamma-beams; expertise in experimental nuclear physics and applications.

\section{Alicja Chruścińska}

Institute of Physics, Faculty of Physics, Astronomy and Informatics, Nicolaus Copernicus University, Toruń, Poland. Specialist in luminescent studies and dating of archaeological artefacts.

\section{Mariaelena Fedi}

National Institute of Nuclear Physics (INFN), Florence, Italy. Physicist/technologist working in applied nuclear physics. Expertise in radiocarbon dating and IBA, initiator of radiocarbon activity in Florence.

\section{Nicla Gelli}

National Institute of Nuclear Physics (INFN), Florence, Italy. Physicist/researcher working in nuclear and applied nuclear physics. Expertise in IBA, external microprobe, nuclear physics.

\section{Lorenzo Giuntini}

University of Florence, Department of Physics, Florence, Italy. Associate professor of Physics. Expertise in external microprobe and IBA, initiator of microbeam activity in Florence.

\section{Novella Grassi}

University of Turin, Department of Physics, Turin, Italy. Physicist working in applied nuclear physics. Expertise in IBA.

\section{Vladimír Hnatowicz and Vladimír Havránek}

Neutron Physics Department, CANAM (Centre for Accelerators and Nuclear Analytical Methods), Nuclear Physics Institute of the Czech Academy of Sciences, Řež, Czech Republic. Ion beam applications for material science, nanostructures deposition and characterisation, microbeam applications, archaeometry, environment studies. Experience in simulation, polymer degradation by irradiation and interaction of energetic ions with materials.

\section{Violeta Iancu}

Extreme Light Infrastructure - Nuclear Physics, Horia Hulubei National Institute for R\&D in Physics and Nuclear Engineering, Bucharest-Magurele, Romania. Junior researcher. Expertise in experimental applied physics, nanostructure deposition and characterisation and numerical simulations.

\section{Mihai Iovea}

ACCENT PRO 2000 s.r.l., Research \& Development Company, Romania. Expert in the field of X-ray imaging, industrial X-ray tomography and X-ray diffraction.

\section{Milko Jaksic}

Ruđer Bošković Institute, Division of experimental physics, Laboratory for ion beam interactions and Centre of excellence for materials and sensing devices (CEMS) Zagreb, Croatia. Senior scientist. Expertise in development and applications of ion microbeam techiques for material modification and analysis.

\section{Zsolt Kasztovszky}

Nuclear Analysis and Radiography Department MTA Centre for Energy Research, Budapest, Hungary. Expertise in prompt gamma analysis after neutron activation.

\section{Anabelle Kriznar}

Centro Nacional de Aceleradores CNA, Universidad de Sevilla, Spain. Interests and expertise: Art History and Hispanic language. Currently a researcher at the University of Seville, analysing $15^{\text {th }}$ and $16^{\text {th }} \mathrm{C}$. panel paintings and polychrome sculpture at the Fine Arts Museum of Seville using non-destructive techniques (UV light, IR spectroscopy, X-Ray fluorescence). 


\section{Jan Kučera}

Neutron Physics Department, Nuclear Physics Institute of the Czech Academy of Sciences, CANAM (Centre of Accelerators and Nuclear Analytical Methods), Rež, Czech Republic. Expertise in nuclear analytical methods, namely neutron activation analysis and its applications in material science, archaeometry, environmental geochemical and biomedical studies, and in preparation and certification of reference materials.

\section{Walter Kutschera}

Emeritus Professor of Physics at the University of Vienna, Austria. Research fields: archaeology, art, atmospheric science, atomic and molecular physics, biomedicine, environmental physics, forensic medicine, Egyptology, geochronology, geomorphology, geophysics, glaciology, groundwater dating, nuclear astrophysics, nuclear physics, oceanography, paleoclimatology.

\section{Lucia Liccioli}

University of Florence, Department of Chemistry, Department of Physics, Florence, Italy. Scientist for cultural heritage. PhD student with expertise in radiocarbon.

\section{Franco Lucarelli}

University of Florence, Department of Physics, Florence, Italy. Associate professor of Physics. Expertise in IBA and environmental studies. Initiator of IBA for aerosol studies in Florence.

\section{Anna Macková}

Neutron Physics Department, CANAM (Centre of Accelerators and Nuclear Analytical Methods), Nuclear Physics Institute of the Czech Academy of Sciences, Řež, Czech Republic. Nuclear physicist working with a wide range of ion beams for material science, nanostructure deposition and characterisation, microbeam applications, archaeometry and environment studies. Associate professor at the J.E. Purkynje University, Usti nad Labem, Czech Republic.

\section{Pier Andrea Mandò}

University of Florence, Department of Physics, Florence, Italy. Professor of Physics working in applied nuclear physics. Expertise in radiocarbon and IBA, initiator of IBA in Florence.

\section{Mirko Massi}

National Institute of Nuclear Physics (INFN), Florence, Italy. Physicist in the field of applied nuclear physics; working position: technologist; expertise in microprobes and IBA, initiator of microbeam activity in Florence.

\section{Anna Mazzinghi}

University of Florence, Department of Physics, scientist for cultural heritage, working position: $\mathrm{PhD}$ student, expertise in diagnostics for $\mathrm{CH}$.

\section{Douglas MacGregor}

University of Glasgow, United Kingdom. Professor of Physics. Vice-chair EPS Nuclear Physics Division; chair EPS committee for IBA prize for Applied Nuclear Science and Nuclear Methods in Medicine; expertise in intermediate energy photonuclear physics.

\section{Alessandro Migliori}

International Atomic Energy Agency, Vienna, Austria. Physicist working in applied nuclear physics; expertise in IBA.

\section{Valentin Moise}

Horia Hulubei National Institute for Nuclear Physics and Engineering, Bucharest, Romania. Experience - gammarays disinfection and radio-polymerisation for wooden artefacts consolidation.

\section{Alessandro Re}

University of Turin, Department of Physics, Turin, Italy. Physicist, research fellow, working in applied material science; expertise in science for cultural heritage.

\section{Miguel Angel Respaldiza}

University of Seville, Sevilla, Spain. Full Professor in Atomic, Molecular and Nuclear Physics Department, Director of the National Accelerator Centre from its creation till 2005, Scientific Adviser to the Centre 2005-2009. Co-ordinator of the "Network Science and Heritage" at the University of Seville. Research in $X$-Ray Fluorescence spectrometry: milli-, micro- and confocalXRF; non-destructive analysis of Cultural Heritage objects, IBA applications in archaeometry, biomedicine, environment and material science. Gamma-ray spectrometry.

\section{László Rosta}

Department of Neutron Spectroscopy, Wigner Research Centre For Physics, Budapest, Hungary. A senior expert in neutron scattering methods (SANS, TOF-ND, etc.).

\section{Chiara Ruberto}

University of Florence, Department of Chemistry, Florence, Italy. Scientist for cultural heritage, working position: $\mathrm{PhD}$ student. Expertise in diagnostics for cultural heritage.

\section{Francisco Javier Santos}

Centro Nacional de Aceleradores CNA, Universidad de Sevilla, Consejo Superior de Investigaciones Científicas CSIC, Junta de Andalucía, Spain. Physicist in the AMS Unit and one of the heads of the Radiocarbon unit. Research fields: the use and technical maintenance of two AMS accelerators (SARA and Micadas) at CNA, since 2005.

\section{Simona Scrivano}

Centro Nacional de Aceleradores CNA, Molecular and Nuclear Physics Department, Universita Sevilla, Spain. The development of portables devices for the analysis of Cultural Heritage and the study of archaeological metallic objects. 


\section{Zdravko Siketić}

Ruđer Bošković Institute, Division of experimental physics, Laboratory for ion beam interactions and Centre of excellence for materials and sensing devices (CEMS) Zagreb, Croatia. Research associate with expertise in development and applications of depth profiling and surface analysis techniques based on ion beams.

\section{Gabriel Suliman}

Extreme Light Infrastructure - Nuclear Physics, Horia Hulubei National Institute for R\&D in Physics and Nuclear Engineering, Bucharest-Magurele, Romania. Junior researcher; expertise in experimental nuclear physics and numerical Monte Carlo simulations.

\section{Žiga Šmit}

Faculty of Mathematics and Physics, University of Ljubljana, and Jožef Stefan Institute, Ljubljana, Slovenia. PIXE,PIGE and microbeam analysis of archaeological artefacts, geological and environmental samples, expert in the field of ion beam analysis of various kinds of archaeological artefacts.

\section{Zoltán Szökefalvi-Nagy}

MTA Wigner Research Centre for Physics, Budapest, Hungery. Research professor emeritus, expertise in development and application of PIXE, XRF and other IBA techniques.

\section{Francesco Taccetti}

National Institute of Nuclear Physics (INFN), Florence, Italy. Physicist; applied nuclear physics and electronic; working position: technologist; expertise in radiocarbon and IBA.

\section{Calin Alexandru Ur}

Extreme Light Infrastructure - Nuclear Physics, Horia Hulubei National Institute for R\&D in Physics and Nuclear Engineering, Bucharest-Magurele, Romania. Senior researcher, Head of Research on High-brilliance gammabeam system; expertise in experimental nuclear physics and particle accelerators.

\section{Wasif Zia}

Institut für Technische und Makromolekulare Chemie (ITMC), RWTH Aachen University, Germany. Physicist studying low-field NMR, instruments, methods, and applications. Coauthored the authoritative monograph "Compact NMR" (De Gruyter Berlin, 2014) and is currently working towards miniaturised NMR sensors for everyday use.

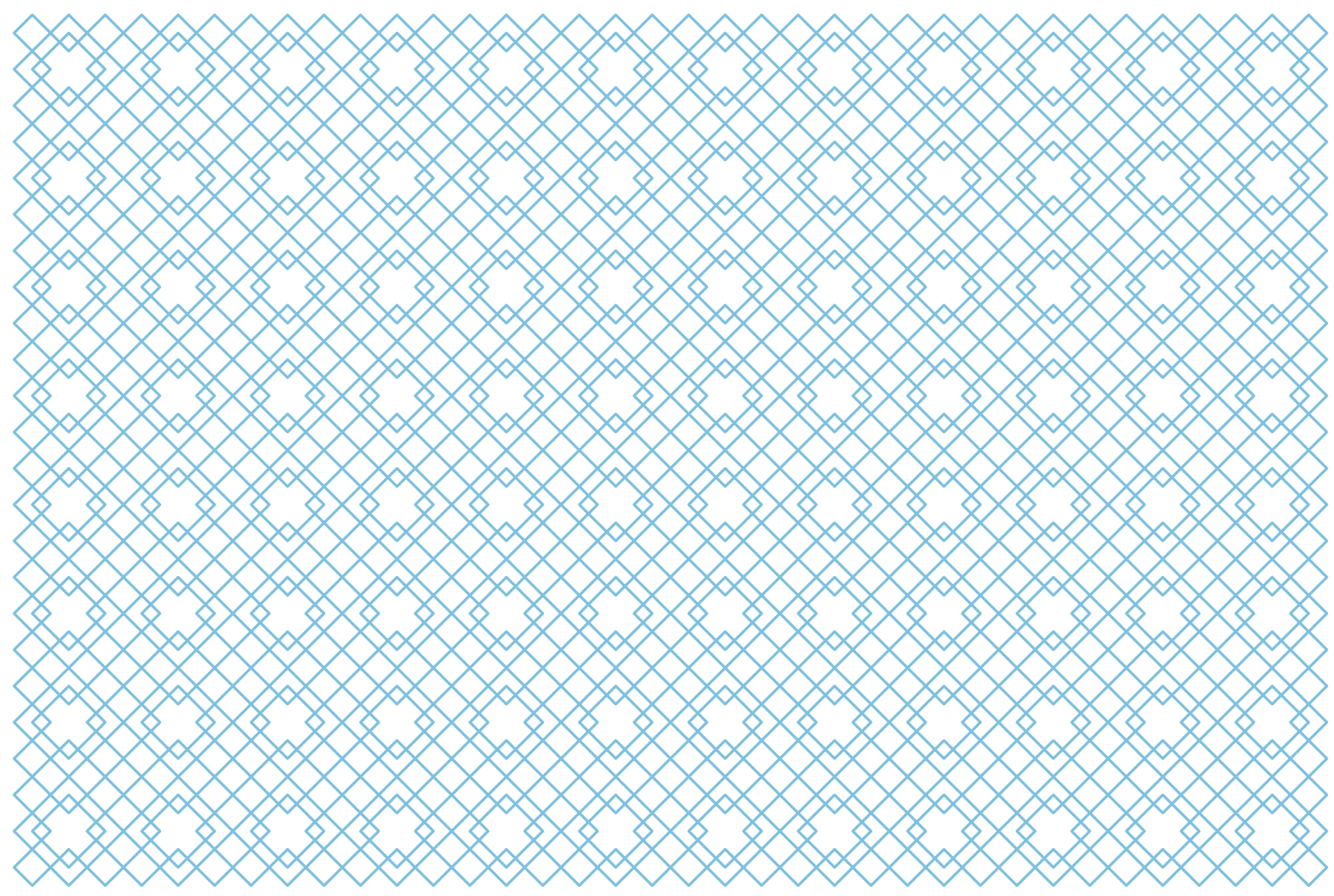




\section{REFERENCES}

\section{FOREWORD}

1. Nuclear Physics for Medicine, Nuclear Physics European Collaboration Committee (NuPECC), 2014, Ed.

F. Azaiez, A. Bracco, J. Dobeš, A. Jokinen, G.E. Körner, A. Maj, A. Murphy, P. Van Duppen, http://www.nupecc.org/npmed/ npmed2014_hires.pdf

2. Energy for the Future: the Nuclear Option, Position Paper of the European Physical Society,
H. Freiesleben, R.C. Johnson, O. Scholten, A. Türler, R. Wyss, 2007

http://c.ymcdn.com/sites/www.eps.org/resource/ resmgr/policy/eps_pp_option_2007.pdf

3. Nuclear Techniques for Cultural Heritage Research, IAEA Radiation Technology Series No. 2, International Atomic Energy Agency, 2011 http://www-pub.iaea.org/MTCD/Publications/PDF/ p1501_web.pdf

\section{CHAPTER 2. ION BEAM ANALYTICAL METHODS}

4. Ion/Neutral Probe Techniques,

A. Macková, A. Pratt, in Handbook of Spectroscopy: Second Edition, Ed. G. Gauglitz, D.S. Moore, Wiley-VCH, 2014, vol. 2, 741-779, ISBN: 978-3-527-32150-6

5. Handbook of Modern Ion-Beam Materials Analysis, Eds. J.R. Tesmer, M. Nastasi, C.J.Maggiore, J.C. Barbour, J.W. Mayer, Materials Research Society, Pittsburgh, PA, 1995.

6. Activation Analysis, Vols I and II, Ed. Z. B. Alfassi, CRC Press, Boca Raton, Florida, USA, 1990, ISBN: 978-0849345845.

7. Use of coulomb excitation by heavy ions $\left({ }^{35} \mathrm{Cl}\right.$, $55 \mathrm{MeV}$ ) for analytical purposes: possibilities and quantitative analysis,

B. Borderie, J.N. Barrrandon, B. Delaunay, M. Basutsu, Nucl. Instrum. Meth. 163 (1979) 441-451 http://dx.doi.org/10.1016/0029-554X(79)90130-7

8. Particle-InducedX-Ray Emission Spectrometry (PIXE), Eds. S.A.E. Johansson, J.L. Campbell and K. G. Malmqvist, John Wiley \& Sons, Inc., New York, 1995, ISBN: 978-0-471-58944-0.

9. Particle-induced gamma-ray emission spectrometry applied to the determination of light elements in individual grains of granite minerals, M. Volfinger and J.L. Robert,
J. Radioanal. Nucl. Chem. 185 (1994) 273-291, http://dx.doi.org/10.1007/BF02041301

10. Ion Beams for Materials Analysis,

Ed. J.R. Bird and S.J. Williams, Academic Press, Sydney, 1989, ISBN: 978-0-12-099740-4.

11. The Heidelberg Proton microprobe, F. Bosch, A. El Goresy, B. Martin, B. Povh, R. Nobiling, D Scwalm, K. Traxel,

Nucl. Instrum. Meth. 149 (1978) 665-668, http://dx.doi.org/10.1016/0029-554X(78)90948-5

12. INAA andPIXE of atmosphericand combustionaerosols, J. Kučera, V. Havranek, J. Smolik, J. Schwarz, V. Vesely, J. Kugler, I. Sykorova, J. Santroch, Biol. Trace Elem. Res. 71-2 (1999) 233-245, http://dx.doi.org/10.1007/BF02784209

13. RBS, PIXE and NDP study of erbium incorporation into glass surface for photonics applications,

A. Mackova, V. Havranek, J. Vacık, L. Salavcova, J. Spirkova,

Nucl. Instrum. Meth. Phys. Res. B, 249 (2006) 856858, http://dx.doi.org/10.1016/j.nimb.2006.03.152

14. RBS/NRA/channeling analysis of implanted immiscible species,

H. Naramoto, S. Yamamoto, K. Narumi, Nucl. Instrum. Meth. Phys. Res. B, 161-163 (2000) 534-538,

http://dx.doi.org/10.1016/S0168-583X(99)00960-X 
15. Modification of surface properties of high and low density polyethylene by Ar plasma discharge, V. Svorcik, K. Kolarova, P. Slepicka, A. Mackova, M. Novotna, V. Hnatowicz,

Polym. Degrad. Stab., 91 (2006) 1219-1225, http://dx.doi.org/10.1016/j.polymdegradstab. 2005.09.007

16. The effects of surface topography in nuclear microprobe Rutherford backscattering analysis, C.P. Hobbs, J.W. McMillan and D.W. Palmer, Nucl. Instrum. Meth. Phys. Res. B, 30 (1988) 342-348, http://dx.doi.org/10.1016/0168-583X(88)90023-7

17. Encyclopedia of Chemical Physics and Physical Chemistry,

N.D. Spencer and J.H. Moore,

Institute of Physics Publishing, Bristol, 1995.

18. Definitive proof of graphene hydrogenation by Clemmensen reduction: use of deuterium labelling, Z. Sofer, O. Jankovský, A. Libánská, P. Šimek, M. Nováček, D. Sedmidubský, A. Macková, R. Mikšováb and M. Pumera,

Nanoscale 7 (2015) 10535: 1-9,

http://dx.doi.org/10.1039/C5NR01356A

19. Materials Analysis by Ion Channeling,

L.C. Feldman, J.W. Mayer and S. T. Picraux, Academic Press, New York, 1982, ISBN: 0-12-252680-5.

20. Characterization of Er:LiNbO ${ }_{3}$ and APE:Er:LiNbO3 by RBS-channeling and XRD techniques,

A. Mackova, R. Groetzschel, F. Eichhorn, P. Nekvindova, J. Spirkova,

Surf. Interface Anal., 36 (2004) 949-951,

http://dx.doi.org/10.1002/sia.1808

21. Characterization of InGaN/GaN heterostructures by means of RBS/channeling,

L. Nowicki, R. Ratajczak, A. Stonert, A. Turos, J.M. Baranowski, R. Banasik, K. Pakula,

Nucl. Instrum. Meth. Phys. Res. B, 161-163 (2000) 539-543,

http://dx.doi.org/10.1016/S0168-583X(99)00840-X

22. Forward Recoil Spectrometry, $J$. Tirira, $Y$. Serruys and P. Trocellier, PlenumPress, New York, 2006, ISBN: 978-1-4613-0353-4.

23. Element-dependent ERDA probing depths using different detection systems,

S. Grigull, U. Kreissig, H. Huber, W. Assmann,

Nucl. Instrum. Meth. Phys. Res. B, 132 (1997) 709-717, http://dx.doi.org/10.1016/S0168-583X(97)00489-8
24. Elemental Analysis by Particle Accelerators, Z.B. Alfassi and M. Peisach, CRC Press, Boca Raton, Florida, 1991, ISBN: 978-0849360312.

25. Using microprobe distribution maps to determine homogeneity and correlation between elements,

F. Munnik, K.A. Sjoland and U. Watjen,

Nucl. Instrum. Meth. Phys. Res. B, 161-163 (2000) 348-353,

http://dx.doi.org/10.1016/S0168-583X(99)00925-8

26. Electron and Ion Microscopy and Microanalysis: Principles and Applications,

L.E. Murr,

Marcel Dekker, New York, 1991,

ISBN: 978-0824785567

27. The beginning of the use of brass in Europe with particular reference to the southeastern Alpine region, J. Istenič, Ž. Šmit,

in Metals and mines: studies in archaeometallurgy, Eds. S. La Niece, D.R. Hook, P.T. Craddock, 2007, 140-147, ISBN: 978-1904982197.

28. The production of brass in Antiquity with particular reference to Roman Britain,

J. Bayley,

in 2000 Years of Zinc and Brass, Ed. P.T. Craddock, British Museum Occasional paper 50, 1990, 7-27, ISBN: 978-0861590506.

29. Archaeometric analysis of Alesia group brooches from sites in Slovenia,

Ž. Šmit, J. Istenič, V. Gerdun, Z. Milič, A. Mladenovič, Arh. Vestnik 56 (2005) 213-233.

30. PIXE analysis of Late La Tène scabbards with nonferrous openwork plates (and associated swords) from Slovenia, Ž. Šmit, J. Istenič, S. Perovšek, Arh. Vestnik 61 (2010) 165-173.

31. Celts and Romans: the contribution of archaeometallurgy to research into cultural interaction, J. Istenič, Ž. Šmit,

in Under the volcano: Proc. Int. Symp. on the Metallurgy of the European Iron Age (SMEIA), Mannheim, Germany, 20 -22 April 2010,

Ed. E. Pernicka, R. Schwab, (Forschungen zur Archäometrie und Altertumwissenschaft, Bd. 5). Rahden/ Westf.: M. Leiderf, 2014, p. 205-220.

32. Plating of archaeological metallic objects - studies by differential PIXE,

Ž. Šmit, J. Istenič, T. Knific, 
Nucl. Instrum. Meth. Phys. Res. B 266 (2008) 2333, http://dx.doi.org/10.1016/j.nimb.2008.03.057

33. External beamline setup for plated target investigation, D. Jezeršek, Ž. Šmit, P. Pelicon,

Nucl. Instrum. Meth. Phys. Res. B 268 (2010) 2006-2009,

http://dx.doi.org/10.1016/j.nimb.2010.02.118

34. Total IBA - Where are we?,

C. Jeynes, M.J. Bailey, N.J. Bright, M.E. Christopher, G.W. Grime, B.N. Jones, V.V. Palitsin, R.P. Webb, Nucl. Instrum. Meth. Phys. Res. B 271 (2012) 107-118, http://dx.doi.org/10.1016/j.nimb.2011.09.020

35. Trade routes across the Mediterranean: a $\mathrm{Sr} / \mathrm{Nd}$ isotopic investigation on Roman colourless glass,

M. Ganio, S. Boyen, D. Brems, R. Scott, D. Foy, K. Latruwe, G. Molin, A. Silvestri, F. Vanhaecke, P. Degryse,

Glass Technology: Eur. J. Glass Sci. Technol. 53(2012) 217-224.

36. Chemical composition of glass beads from the necropolis of Apollonia Pontica $\left(5^{\text {th }}-3^{\text {rd }}\right.$ C. BC),

V. Ljubomirova, Ž. Šmit, H. Fajfar, I. Kuleff,

Archaeologia Bulgarica 18 (2014) 1-17.

37. Analysis of Roman glass from Albania by PIXEPIGE method,

Ž. Šmit, F. Tartari, F. Stamati, A. Vevecka-Priftaj, J. Istenič,

Nucl. Instrum. Meth. Phys. Res. B 296 (2013) 7-13, http://dx.doi.org/10.1016/j.nimb.2012.12.007

38. Characterization of the chemical composition of archaeological glass finds from South-Eastern Bulgaria using PIXE, PIGE and ICP-AES,

D. Lesigyarski, Ž. Šmit, B. Zlateva-Rangelova, K. Koseva, I. Kuleff,

J. Radioanal. Nucl. Chem. 295 (2013) 1605-1619, http://dx.doi.org/10.1007/s10967-012-2326-5

39. PIXE-PIGE investigation of Roman Imperial vessels and window glass from Mt. Kosmaj, Serbia (Moesia Superior),

M.M. Stojanović, Ž. Šmit, M. Glumac, J. Mutić,

J. Arch. Sci. Reports 1 (2015) 53-63, http://dx.doi.org/10.1016/j.jasrep.2014.11.001

40. A raw glass chunk from the vicinity of Nauportus (Vrhnika),

J. Istenič, Ž. Šmit,

in Emona: between Aquileia and Pannonia,

Ed. B. Županek, I. Lazar,

Annales Mediterranei, Koper (2012) 301-309.
41. Analysis of glass from the post-Roman settlement Tonovcov grad (Slovenia)

by PIXE-PIGE and LA-ICP-MS,

Ž. Šmit, T. Milavec, H. Fajfar, Th. Rehren, J.W. Lankton, B. Gratuze,

Nucl. Instrum. Meth. Phys. Res. B 311 (2014) 53-59, http://dx.doi.org/10.1016/j.nimb.2013.06.012

42. Analysis of early medieval glass beads - Glass in the transition period,

Ž. Šmit, T. Knific, D. Jezeršek, J. Istenič,

Nucl. Instrum. Meth. Phys. Res. B 278 (2012) 8-14, http://dx.doi.org/10.1016/j.nimb.2012.01.044

43. PIXE-PIGE characterization of medieval glass, Ž. Šmit, P. Pelicon, M. Holc, M Kos,

Nucl. Instrum. Meth. Phys. Res. B 189 (2002) 344, http://dx.doi.org/10.1016/S0168-583X(01)01084-9

44. Spread of façon-de-Venise glassmaking through central and western Europe,

Ž. Šmit, K. Janssens, O. Schalm, M. Kos,

Nucl. Instrum. Meth. Phys. Res. B 213 (2004) 717, http://dx.doi.org/10.1016/S0168-583X(03)01691-4

45. Trace element fingerprinting of façon-de-Venise glass,

Ž. Šmit, K. Janssens, E. Bulska, B. Wagner, M. Kos, I. Lazar,

Nucl. Instrum. Meth. Phys. Res. B 239 (2005) 94, http://dx.doi.org/10.1016/j.nimb.2005.06.182

46. Evidence of early medieval soda ash glass in the archaeological site of San Genesio (Tuscany),

S. Cagno, L. Favaretto,M.Mendera, A. Izmer,F. Vanhaecke, K. Janssens,

J. Arch. Sci. 39 (2012) 1540-1552,

http://dx.doi.org/10.1016/j.jas.2011.12.031

47. Analysis of Venetian type glass fragments from the ancient city of Lezha (Albania),

Ž. Šmit, F. Stamati, N. Civici, A. Vevecka-Priftaj, M. Kos, D. Jezeršek,

Nucl. Instrum. Meth. Phys. Res. B 267 (2009) 2538, http://dx.doi.org/10.1016/j.nimb.2009.05.048

48. PIXE-PIGE analysis of colored historic glass, H. Fajfar, Ž. Šmit, M. Kos,

Glass Technol.: Eur. J. Glass Sci. Technol. A 54 (2013) 218-225.

49. PIXE-PIGE analysis of $18^{\text {th }}$ and early $19^{\text {th }}$ century creamware from Slovenia and Northern Italy,

M. Kos, Ž. Šmit,

J. Cult. Herit. 12 (2011) 236-242,

http://dx.doi.org/10.1016/j.culher.2010.12.010 
50. PIXE/PIGE characterisation of emeralds using an external micro-beam,

T. Calligaro, J.-C. Dran, J.-P. Poirot, G. Querré, J. Salomon, J.C. Zwaan,

Nucl. Instrum. Meth. Phys. Res. B 161-163 (2000) 769-774,

http://dx.doi.org/10.1016/S0168-583X(99)00974-X

51. The origin of emeralds embedded in archaeological artefacts in Slovenia,

A. Kržič, Ž. Šmit, H. Fajfar, M. Dolenec, B. Činč Juhant, M. Jerše,

Geologija 56 (2013) 29-46,

http://dx.doi.org/10.5474/geologija.2013.003

52. Combined external-beam and $\mu$-Raman characterization of garnets used in Merovingian jewelry,

T. Calligaro, S. Colinart, J.-P. Poirot, C. Sudres,

Nucl. Instrum. Meth. Phys. Res. B 189 (2002) 320-327,

http://dx.doi.org/10.1016/S0168-583X(01)01078-3

53. Contribution à l'étude des grenats mérovingiens (Basilique de Saint-Denis et autres collections du musée d'Archéologie nationale, diverses collections publiques et objets de fouilles récentes),

T. Calligaro, P. Perin, F. Vallet, J.-P. Poirot,

Antiquités nationales 38 (2006-2007) 111-144.

54. Study of the provenance of Belgian Merovingian garnets by PIXE at IPNAS cyclotron,

F. Mathis, O. Vrielynck, K. Laclavetine, G. Chêne, D. Strivay,

Nucl. Instrum. Meth. Phys. Res. B 266 (2008) 23482352, http://dx.doi.org/10.1016/j.nimb.2008.03.055

55. Analysis of garnets from the archaeological sites in Slovenia,

Ž. Šmit, H. Fajfar, M. Jeršek, T. Knific, J. Lux,

Nucl. Instrum. Meth. Phys. Res. B 328 (2014) 89-94,

http://dx.doi.org/10.1016/j.nimb.2014.02.121

56. Concentration profiles in paint layers studied by differential PIXE,

Ž. Šmit, M. Uršič, P. Pelicon, T. Trček-Pečak, B. Šeme, A. Smrekar, I. Langus, I. Nemec, K. Kavkler,

Nucl. Instrum. Meth. Phys. Res. B 266 (2008) 2047-2059

http://dx.doi.org/10.1016/j.nimb.2008.03.191

57. Analysis of iron gall inks by PIXE,

M. Budnar, M. Uršič, J. Simčič, P. Pelicon, J. Kolar, V.S. Šelih, M. Strlič,

Nucl. Instrum. Meth. Phys. Res. B 243 (2006) 407-416, http://dx.doi.org/10.1016/j.nimb.2005.10.013
58. Proton microscopy on 3 MV Tandetron Accelerator at NPI Řež.

P. Malinský, V. Havránek, V. Peřina, V. Voseček, K. Kranda,

in Mikroskopie 2012. Praha 4: Československá mikroskopická společnost, 2012. S. 16-16.

59. Determination of $\mathrm{As}, \mathrm{Cd}, \mathrm{Cu}, \mathrm{Hg}, \mathrm{Mo}, \mathrm{Sb}$, and $\mathrm{Se}$ in biological reference materials by radiochemical neutron activation analysis,

J. Kučera, L. Soukal,

J. Radioanal. Nucl. Chem. 168 (1993) 185-99, http://dx.doi.org/10.1007/BF02040891

60. Was He Murdered Or Was He Not?-Part l: Analyses of Mercury in the Remains of Tycho Brahe,

K. L. Rasmussen, J. Kučera, L. Skytte, J. Kameník,

V. Havránek, J. Smolík, P. Velemínský, N. Lynnerup,

J. Bruzek, J. Vellev,

Archaeometry 55 (2013) 1187-1195,

http://dx.doi.org/10.1111/j.1475-4754.2012.00729.x

61. The Sarmizegetusa Bracelets,

B. Constantinescu, E. Oberländer-Târnoveanu, R. Bugoi, V. Cojocaru, M. Radtke,

Antiquity Journal, London, Volume 84 (2010) 1028-1042,

http://dx.doi.org/10.1017/S0003598X00067053

62. Elemental analysis through X-ray techniques applied in archaeological gold authentication - the case of Transylvanian gold and of the Dacian bracelets, B. Constantinescu, R. Bugoi, V. Cojocaru, R. Simon, D. Grambole, F. Munnik, E. Oberländer-Târnoveanu, Spectrochimica Acta B 64 (2009) 1198-1203, http://dx.doi.org/10.1016/j.sab.2009.08.007

63. Studies on archaeological gold items found in Romanian territory using X-Ray-based analytical spectrometry,

B. Constantinescu, A. Vasilescu, D. Stan, M. Radtke, G. Buzanich, D. Ceccato, U. Reinholz, E. OberlaenderTarnoveanu,

J. Anal. At. Spectrom. 27 (2012) 2076-2081, 2012, http://dx.doi.org/10.1039/c2ja30158j

64. SR-XRF and micro-PIXE studies on ancient metallurgy of thirteen Dacian gold bracelets,

B. Constantinescu, A. Vasilescu, M. Radtke, U. Reinholz, Appl. Phys. A 109 (2012) 395-402, http://dx.doi.org/10.1007/s00339-012-7306-7

65. Lapis lazuli from Sar-e-Sang, Badakhshan, Afghanistan,

J. Wyart, P. Bariand, J. Filippi,

Gems \& Gemmology 17 (1981) 184-190. 
66. The ionoluminescence apparatus at the LABEC external microbeam facility,

S. Calusi, E. Colombo, L. Giuntini, A. Lo Giudice, C. Manfredotti, M. Massi, G. Pratesi, E. Vittone, Nucl. Instrum. Meth. Phys. Res. B 266 (2008) 2306-2310,

http://dx.doi.org/10.1016/j.nimb.2008.03.077

67. Recent developments of ion beam induced luminescence at the external scanning microbeam facility of the LABEC laboratory in Florence, E. Colombo, S. Calusi, R. Cossio, L. Giuntini, A. Lo Giudice, P.A. Mandò, C. Manfredotti, M. Massi, M.A. Mirto, E. Vittone,

Nucl. Instrum. Meth. Phys. Res. B 266 (2008) 1527-1532,

http://dx.doi.org/10.1016/j.nimb.2007.11.067

68. Multitechnique characterization of lapis lazuli for provenance study,

A. Lo Giudice, A. Re, S. Calusi, L. Giuntini, M. Massi, P. Olivero, G. Pratesi, M. Albonico, E. Conz,

Anal. Bioanal. Chem. 395 (2009) 2211-2217,

http://dx.doi.org/10.1007/s00216-009-3039-7

69. Lapis lazuli provenance study by means of micro-PIXE, A. Re, A. Lo Giudice, D. Angelici, S. Calusi, L. Giuntini, M. Massi, G. Pratesi,

Nucl. Instrum. Meth. Phys. Res. B, 269(20) (2011) 2373-2377,

http://dx.doi.org/10.1016/j.nimb.2011.02.070

70. New markers to identify the provenance of lapis lazuli: trace elements in pyrite by means of micro-PIXE,

A. Re, D. Angelici, A. Lo Giudice, E. Maupas, L. Giuntini,

S. Calusi, N. Gelli,M. Massi, A. Borghi, L.M. Gallo,

G. Pratesi. P.A. Mandò,

Appl. Phys. A, 111(1) (2013) 69-74,

http://dx.doi.org/10.1007/s00339-013-7597-3

71. Ion Beam Analysis for the provenance attribution of lapis lazuli used in glyptic art: The case of the "Collezione Medicea",

A. Re, D. Angelici, A. Lo Giudice, J. Corsi, S. Allegretti, A.F. Biondi, G. Gariani, S. Calusi, N. Gelli, L. Giuntini, M. Massi, F. Taccetti, L. La Torre, V. Rigato, G. Pratesi, Nucl. Instrum. Meth. Phys. Res. B, 348 (2015) 278-284, http://dx.doi.org/10.1016/j.nimb.2014.11.060

72. $\mu$-XRF Analysis of Trace Elements in Lapis LazuliForming Minerals for a Provenance Study,

D. Angelici, A. Borghi, F. Chiarelli, R. Cossio, G. Gariani,

A. Lo Giudice, A. Re, G. Pratesi, G. Vaggelli,

Microscopy and Microanalysis, 21(2) (2015) 526-533

http://dx.doi.org/10.1017/S143192761500015X
73. Mineralogical characterization of the blue pigment of Michelangelo's fresco "The Last Judgment",

P. Ballirano, A. Maras,

American Mineralogist 91 (2006) 997-1005, http://dx.doi.org/10.2138/am.2006.2117

74. Where does lapis lazuli come from? Non-destructive provenance analysis by PGAA,

J. Zoldfoldi, S. Richter, Zs. Kasztovszky, J. Mihaly,

in Proc. 34 ${ }^{\text {th }}$ Int. Symp. on Archaeometry (Zaragoza, Spain, 2004) 2006 353-361,

http://ifc.dpz.es/recursos/publicaciones/26/10/_ ebook.pdf, ISBN: 84-7820-848-8

75. $\mathrm{CO}_{2}$ entrapment in natural ultramarine blue, C. Miliani, A. Daveri, B.G. Brunetti, A. Sgamellotti, Chem. Phys. Lett. 466 (2008) 148-151, http://dx.doi.org/10.1016/j.cplett.2008.10.038

76. An integrated spectroscopic approach for the identification of what distinguishes Afghan lapis lazuli from others,

M. Bacci, C. Cucci, E. Del Federico, A. Ienco, A. Jerschow, J.M. Newman, M. Picollo,

Vibrational Spect. 49 (2009) 80-83,

http://dx.doi.org/10.1016/j.vibspec.2008.05.002

77. Characterization of Lapis Lazuli Pigments Using a Multitechnique Analytical Approach: Implications for Identification and Geological Provenancing, C.M. Schmidt, M.S. Walton, K. Trentelman, Anal. Chem. 81 (2009) 8513-8518, http://dx.doi.org/10.1021/ac901436g

78. Advances in elemental imaging of rocks using the AGLAE external microbeam,

T. Calligaro, Y. Coquinot, L. Pichon, B. Moignard,

Nucl. Instrum. Meth. Phys. Res. B269 (2011)2364-2372, http://dx.doi.org/10.1016/j.nimb.2011.02.074

79. Characterization of lapis lazuli and corresponding purified pigments for a provenance study of ultramarine pigments used in works of art,

M. Favaro, A. Guastoni, F. Marini, S. Bianchin, A. Gambirasi,

Analytical and Bioanalytical Chemistry 402 (2012) 2195-2208.

http://dx.doi.org/10.1007/s00216-011-5645-4

80. Characterization of the lapis lazuli from the Egyptian treasure of Tôd and its alteration using external $\mu$-PIXE and $\mu$-IBIL

T. Calligaro, Y. Coquinot, L. Pichon, G. PierratBonnefois, P. de Campos, A. Re, D. Angelici,

Nucl. Instrum. Meth. Phys. Res. B 318 (2014) 139-144, http://dx.doi.org/10.1016/j.nimb.2013.06.063 
81. In-air broad beam ionoluminescence microscopy as a tool for rocks and stone artworks characterisation, A. Lo Giudice, A. Re, D. Angelici, S. Calusi, N. Gelli, L. Giuntini, M. Massi, G. Pratesi, Anal. Bioanal. Chem. 404 (2012) 277-281, http://dx.doi.org/10.1007/s00216-012-6110-8

82. Differential PIXE for investigating the layer structure of paintings,

P.A. Mando, M.E. Fedi, N. Grassi, A. Migliori,

Nucl. Instrum. Meth. Phys. Res. B, 239 (2005) 71-76, http://dx.doi.org/10.1016/j.nimb.2013.06.063

83. Identification of lapis-lazuli pigments in paint layers by PIGE measurements,

N. Grassi, A. Migliori, P.A. Mando, H. Calvo del Castillo, Nucl. Instrum. Meth. Phys. Res. B 219-220 (2004) 48-52,

http://dx.doi.org/10.1016/j.nimb.2004.01.026

84. Differential and scanning-mode external PIXE for the analysis of the painting "Ritratto Trivulzio" by Antonello da Messina,

N. Grassi,

Nucl. Instrum. Meth. Phys. Res. B 267 (2009) 825, http://dx.doi.org/10.1016/j.nimb.2008.12.018

85. Galileo's writings: chronology by PIXE,

L. Giuntini, F. Lucarelli, P.A. Mandò, W. Hooper, P.H. Barker,

Nucl. Instrum. Meth. Phys. Res. B 95 (1995) 389,
http://dx.doi.org/10.1016/0168-583X(94)00538-9

86. Further results from PIXE analysis of inks in Galileo's notes on motion,

P. Del Carmine, L. Giuntini, W. Hooper, F. Lucarelli, P.A. Mandò,

Nucl. Instrum. Meth. Phys. Res. B 113 (1996) 354-358,

http://dx.doi.org/10.1016/0168-583X(95)01335-0

87. Applied nuclear physics in the final years at Arcetri and the emergence of new activities at the LABEC of the Polo Scientifico at Sesto Fiorentino,

P.A. Mandò

Il Colle di Galileo, 3, (2014) 15-29,

http://dx.doi.org/10.13128/Colle_Galileo-14645

88. Development of a time-of-flight spectrometer at the Ruđer Bošković Institute in Zagreb,

Z. Siketić, I. Bogdanović Radović, M. Jakšić,

Nucl. Instrum. Meth. Phys. Res. B 266 (2008) 1328-1332,

http://dx.doi.org/10.1016/j.nimb.2007.12.070

89. Potku - New analysis software for heavy ion elastic recoil detection analysis,

K. Arstila, J. Julin, M.I. Laitinen, J. Aalto, T. Konu, S. Kärkkäinen, S. Rahkonen, M. Raunio, J. Itkonen, J.-P. Santanen, T. Tuovinen, T. Sajavaara,

Nucl. Instrum. Meth. Phys. Res. B 331 (2014) 34-41 http://dx.doi.org/10.1016/j.nimb.2014.02.016

\section{CHAPTER 3. NEUTRON BEAM ANALYTICAL METHODS}

90. Principles of PGAA method,

Zs. Révay, T. Belgya,

in Handbook of Prompt Gamma Activation Analysis with Neutron Beams,

Ed. G.L. Molnár, Kluwer Academic Publishers, Dordrecht, 2004, pp. 1-30,

ISBN: 978-1402013041

91. Neutronsources.org website

http://neutronsources.org/

92. The NPI cyclotron-based fast neutron facility, P. Bém, V. Burjan, J. Dobeš, U. Fisher, M. Gotz, M. Honusek, V. Kroha, J. Novák, S.P. Simakov, E. Šimečková,

in ND 2007, Int.. Conf. on Nuclear Data for Science and Technology, April 2007, Nice, France,

Ed. O.Bersillon, F.Gunsing, E.Bauge, R.Jacqmin, S.Leray,

EDP Sciences, 2008, Vol. 1 pp. 555-558, ISBN: 978-2-7598-0090-2
93. Beams and Facilities,

R.M. Lindstrom, Zs. Révay,

in Handbook of Prompt Gamma Activation Analysis with Neutron Beams,

Ed. G.L. Molnár, Kluwer Academic Publishers, Dordrecht, 2004, pp. 31-58,

ISBN: 978-1402013041

94. How Can Neutrons Contribute to Cultural Heritage Research?,

Zs. Kasztovszky, L. Rosta,

Neutron News 23:1, 2012, 25-28,

http://dx.doi.org/10.1080/10448632.2012.645689

95. Applications of PGAA with Neutron Beams,

D.L. Anderson, Zs. Kasztovszky,

in Handbook of Prompt Gamma Activation Analysis with Neutron Beams,

Ed. G.L. Molnár, Kluwer Academic Publishers, Dordrecht, 2004,pp. 137-172,

ISBN: 978-1402013041 
96. Neutron depth profiling: overview and description of NIST facilities,

R.G. Downing, G.P. Lamaze, J.K. Langland, S.T. Hwang, NIST J. Res. 98 (1993) 109,

http://dx.doi.org/10.6028/jres.098.008

97. Neutron depth profiling,

D. Fink,

Hahn-Meitner Institute Berlin, Report HMI-B 539

(1996) 1-314, ISSN 0936-0891

98. Diffusion of ${ }^{6} \mathrm{Li}$ in $\mathrm{Ta}$ and $\mathrm{W}$,

J. Vacik, V. Hnatowicz, U. Koester,J. Červená, V. Havránek, G. Pasold,

Nucl. Instrum. Meth. Phys. Res. Meth. B 24(2006) 865868, http://dx.doi.org/10.1016/j.nimb.2006.03.153

99. Applications of $(n, p)$ and $(n, a)$ reactions and a backscattering technique to fusion reactor materials, archeometry, and nuclear spectroscopy,

D. Fink, J.P. Biersack, H. Grawe, J. Riederer, K. Mueller, R. Henkelmann,

Nucl. Instrum. Meth. 168 (1980) 453-457, http://dx.doi.org/10.1016/0029-554X(80)91293-8

100. Cold neutron prompt gamma activation analysis - a non-destructive method for characterisation of high silica content chipped stone tools and raw materials, Z. Kasztovszky, K. T. Biró, A. Markó, V. Dobosi, Archaeometry 50 (2008) 12-29, http://dx.doi.org/10.1111/j.1475-4754.2007.00348.x

101. Kora-neolitikus radiolarit és obszidián kőeszközök vizsgálata prompt-gamma aktivációs analízissel (in Hungarian),

Zs. Kasztovszky, T. Težak-Greg,

in Őskoros Kutatók 6. Összejövetele Konferencia Kötet, Ed. I. Gábor, Szombathely, 2009, pp. 189-195.

102. Incipient archaeometry in Venezuela: Provenance study of pre-Hispanic pottery figurines,

L. Sajo-Bohus, M.M. Mackowiak de Antczak, E.D. Greaves, A. Antczak, J. Bermudez, Zs. Kasztovszky, T. Poirier, A. Simonits,
J. Radioanal. and Nucl. Chem. 265 (2005) 247-256, http://dx.doi.org/10.1007/s10967-005-0816-4

103. Early Neolithic pottery and its environment in Hungary, H. Taubald, K.T. Biró, Zs. Kasztovszky, M. Balla, in Proc. $36^{\text {th }}$ Int. Symp. on Archaeometry, Eds. J.F. Moreau, R. Auger, J. Chabot and A. Herzog, Cahiers d'archéologie du CELAT, No. 25, Série archéométrie no. 7, (2009) pp. 237-241, ISBN 978 2-920576-98-8

104. Petro-mineralogy and geochemistry as tools of provenance analysis on archaeological pottery: Study of Inka Period ceramics from Paria, Bolivia, V. Szilágyi, J. Gyarmati, M. Tóth, H. Taubald, M. Balla, Zs. Kasztovszky, Gy. Szakmány, J. South American Earth Sciences 36 (2012) 1-17, http://dx.doi.org/10.1016/j.jsames.2011.11.001

105. Archaeometric investigation of red-figure pottery fragments from Gioiosa Guardia (Messina, Sicily) by INAA, FT-IR and TOF-ND techniques,

D. Barilaro, V. Crupi, S. Interdonato, F. Longo, G. Maisano, D. Majolino, V. Venuti, G. Barone, P. Mazzoleni, T. Tigano, S. Imberti and W. Kockelmann, II Nuovo Cimento C 31, (2008) 371-388, http://dx.doi.org/10.1393/ncc/i2008-10297-1

106. Comparative archaeometrical study of Roman silver coins by prompt gamma activation analysis and SEM-EDX,

Zs. Kasztovszky, E. Panczyk, W. Fedorowicz, Zs. Révay, B. Sartowska,

J. Radioanal. Nucl. Chem. 265 (2005) 193-199, http://dx.doi.org/10.1007/s10967-005-0809-3

107. 5,000 years old Egyptian iron beads made from hammered meteoritic iron,

T. Rehren, T. Belgya, A. Jambon, G. Káli, Zs. Kasztovszky, Z. Kis, I. Kovács, B. Maróti, M. MartinónTorres, G. Miniaci, V.C. Pigott, M. Radivojevi, L. Rosta, L. Szentmiklósi, Z. Szőkefalvi-Nagy,

J. Arch. Sci. 40 (2013) 4785-4792, http://dx.doi.org/10.1016/j.jas.2013.06.002

\section{CHAPTER 4. ACCELERATOR MASS SPECTROMETRY AND LUMINESCENT DATING METHODS}

108. Thermoluminescence Dating, M.J. Aitken, Academic Press, London, 1985, ISBN: 978-0120463817

109. Partial matrix doses for thermoluminescence dating, H.L. Oczkowsk, K. Przegiętka, Physica Scripta 58 (1998) 534-53, http://dx.doi.org/10.1088/0031-8949/58/5/019
110. Dose-rate conversion factors: update, G. Guérin, N. Mercier, G. Adamiec, Ancient TL, 29 (2011) 5-8, http://www.ecu.edu/cs-cas/ physics/Ancient-Timeline/upload/ATL_29-1-Guerin.pdf

111. Radiocarbon Dating,

W.F. Libby,

University of Chicago Press, Chicago 1952. 
112. A high sensitivity optically stimulated luminescence scanning system for measurement of single sandsized grains,

G.A.T. Duller. L. Bøtter-Jensen, P. Kohsiek, A.S. Murray, Radiat. Prot. Dosim. 84 (1999) 325-330,

https://rpd.oxfordjournals.org/content/84/1-4/325. full.pdf+html

113. Radiation in archaeometry: archaeological dating, M. Martini, E. Sibilia,

Rad. Phys. Chem. 61 (2001) 241-246.

http://dx.doi.org/10.1016/S0969-806X(01)00247-X

114. A review of quartz optically stimulated luminescence characteristics and their relevance in single-aliquot regeneration dating protocol,

A.G. Wintle, A.S. Murray,

Rad. Meas. 41 (2006) 369-391,

http://dx.doi.org/10.1016/j.radmeas.2005.11.001

115. MICADAS: a new compact radiocarbon AMS system, H.A. Synal, M. Stocker, M. Suter,

Nucl. Instrum. Meth. Phys. Res. Meth. B 259 (2007) 7-13, http://dx.doi.org/10.1016/j.nimb.2007.01.138

116. Über die Datierung von Keramik und Ziegel durch Thermolumineszenz (in German),

N. Grogler, F.G. Houtermans, H. Stauffer,

Helvetica Physica Acta 33 (1960) 595-596.

117. Dating by thermoluminescence,

G.C. Kennedy, L. Knopf,

Archaeology 13 (1960) 147-148.

118. Methodological developments in the luminescence dating of brick from English late-medieval and postmedieval buildings,

I.K. Bailiff,

Archaeometry 49 (2007) 827-851,

http://dx.doi.org/10.1111/j.1475-4754.2007.00338.x

119. Thermoluminescence analysis in historical architecture: application to the ornamentations "in cotto" of the atrium of the Abbey of Pomposa,

F. Bevilacqua, C. Chiavari, C. Di Francesco, E. Migliorini, M. Martini, E. Sibilia,

in $6^{\text {th }}$ Int. Conf. on Non Destructive Testing and Microanalysis for the Diagnostics and Conservation of the Cultural and Environmental Heritage, Rome, 1999.

120. Combined dating methods applied to building archaeology: The contribution of thermoluminescence to the case of the bell tower of St Martin's church, Angers (France),

S. Blain, P. Guibert, D. Prigent, P. Lanos, C. Oberlin, C Sapin, A. Bouvier, P. Dufresne,
Geochronometria 38 (2011) 55-63,

http://dx.doi.org/10.2478/s13386-011-0010-0

121. TL-dating applied to building archaeology: The case of the medieval church Notre-Dame-Sous-Terre (Mont-Saint-Michel, France),

S. Blain, P. Guibert, A. BouvierE. Vieillevigne, F. Bechtel, C. Sapin, M. Baylé,

Rad. Meas. 42 (2007) 1483-1491,

http://dx.doi.org/10.1016/j.radmeas.2007.07.015

122. An intercomparison study of luminescence dating protocols and techniques applied to medieval brick samples from Normandy (France),

S. Blain, I.K. Bailiff, P. Guibert, A. Bouvier, M. Baylé, Quaternary Geochronology 5 (2010) 311-316, http://dx.doi.org/10.1016/j.quageo.2009.02.016

123. Luminescence dating of bricks from the gothic Saint James church in Toruń,

A. Chruścińska, A. Cicha, N. Kijek, P.Palczewski, K.R. Przegiętka, K. Sulkowska-Tuszyńska, Geochronometria 41 (2014) 352 - 360, http://dx.doi.org/10.2478/s13386-013-0165-y

124. Thermoluminescence dating in architectural history: Venetian villas,

C. Goedicke, K.Slusallek, M. Kubelik, J. Soc. Architectural Historians 40 (1981) 203-217, http://dx.doi.org/10.2307/989694

125. Luminescence dating of architectural ceramics from an early medieval abbey: The St Philbert Intercomparison (Loire Atlantique, France),

P. Guibert, I.K. Bailiff, S. Blain, A.M. Gueli AM, M. Martini, E. Sibilia, G. Stell, S.O. Troja,

Radiation Measurements 44 (2009) 488-493,

http://dx.doi.org/10.1016/j.radmeas.2009.06.006

126. The Chronology of the neolithic sequence at Dikili Tash, Macedonia, Greece: TL dating of domestic ovens,

C. Roque, P. Guibert, E. Vartanian, F. Bechtel, R. Treuil, P. Darcque, H. Koukouli-Chryssanthaki, D. Malamidou, Archaeometry 44 (4) (2002) 613-633, http://dx.doi.org/10.1111/1475-4754.t01-1-00089

127. Dependence of luminescence characteristics of irradiated quartz with thermal treatment and consequences for TL-dating: Application to Dating of Solutrean heated quartz pebbles from Laugerie Haute (Dordogne, France),

C. Roque, P. Guibert, M. Duttine, E. Vartania, R. Chapoulie, F. Bechtel,

Geochronometria 23 (2004) 1-8,

http://www.geochronometria.pl/geo_23.html 
128. Discovering forgeries of modern art by the ${ }^{14} \mathrm{C}$ bomb peak,

L. Caforio, M. E. Fedi, P. A. Mando, F. Minarelli, E. Peccenini, V. Pellicori, F. C. Petrucci, P. Schwartzbaum, F. Taccetti,

Eur. Phys. J. Plus, 129 (2014) 1-5,

http://dx.doi.org/10.1140/epjp/i2014-14006-6

129. The external scanning proton microprobe of Firenze:

A comprehensive description,

L. Giuntini, M. Massi, S. Calusi,

Nucl. Instrum. Meth. A 576 (2007) 266-273,

http://dx.doi.org/10.1016/j.nima.2007.03.021
130. The external beam microprobe facility in Florence: Set-up and performance,

M. Massi, L. Giuntini, M. Chiari, N. Gelli, P.A. Mandò, Nucl. Instrum. Meth. Phys. Res. B 190 (2002) 276-282, http://dx.doi.org/10.1016/S0168-583X(01)01212-5

131. The Artemidorus papyrus: solving an ancient puzzle with radiocarbon and Ion Beam Analysis measurements,

M.E. Fedi, L. Carraresi, N. Grassi, A. Migliori, F. Taccetti, F. Terrasi, P.A. Mandò,

Radiocarbon 52 (2010) 356-363 https://journals.uair. arizona.edu/index.php/radiocarbon/issue/view/186

\section{CHAPTER 5. COMPLEMENTARY ANALYTICAL METHODS - GAMMA-BEAM METHODS, XRF AND NMR}

132. Nondestructive determination of the ${ }^{13} \mathrm{C}$ content in isotopic diamond by nuclear resonance fluorescence, O. Beck, T. Ruf, Y. Finkelstein, M. Cardona, T. R. Anthony, D. Belic, T. Eckert, D. Jäger, U. Kneissl, H. Maser, R. Moreh, A. Nord, H. H. Pitz and A. Wolpert, J. Appl. Phys. 83 (1998) 5484-5488. http://dx.doi.org/10.1063/1.367378

133. Nondestructive detection of hidden chemical compounds with laser Compton-scattering gamma rays,

T. Hayakawa, H. Ohgaki, T. Shizuma, R. Hajima, N. Kikuzawa, E. Minehara, T. Kii, H. Toyokawa, Rev. Sci. Instrum. 80 (2009) 045110, http://dx.doi.org/10.1063/1.3125022

134. Two-Dimensional isotope imaging of radiation shielded materials using nuclear resonance fluorescence, H. Toyokawa, H. Ohgaki, T. Hayakawa, T. Kii, T. Shizuma, R. Hajima, N. Kikuzawa, K. Masuda, F. Kitatani, H. Harada,

Jap. J. Appl. Phys. 50 (2011) 100209: 1-3

http://dx.doi.org/10.1143/JJAP.50.100209

135. Transmission-based detection of nuclides with nuclear resonance fluorescence using a quasimonoenergetic photon source, C.A. Hagmann, J.M. Hall, M.S. Johnson, D.P. McNabb, J.H. Kelley, C. Huibregtse, E. Kwan, G. Rusev, A.P. Tonchev,

J. Appl. Phys. 106 (2009) 084901, http://dx.doi.org/10.1063/1.3238328

136. Gamma-beam industrial applications at ELI-NP, G. Suliman, V. Iancu, C. A. Ur, M. Iovea, I. Daito, H. Ohgaki,

Romanian Reports in Physics (2016) in press, http://www.rrp.infim.ro/
137. Technical Design Report EuroGammaS proposal for the ELI-NP Gamma beam System,

0 . Adriani et al., arXiv:1407.3669, http://arxiv.org/abs/1407.3669

138. Fundamentals of computerized tomography: Image reconstruction from projection,

G.T. Herman

$2^{\text {nd }}$ edition, Springer, Heidelberg (2009), ISBN: 978-1852336172

139. Simulation Study on Computer Tomography Imaging of Nuclear Distribution by Quasi Monoenergetic Gamma Rays with Nuclear Resonance Fluorescence: case study for ELI-NP application,

I. Daito, H. Ohgaki, G. Suliman, V. Iancu, C.A. Ur, M. lovea,

Energy Precedia (to be published).

140. Handbook of Practical X-Ray Fluorescence, Eds.B.Beckhoff, B. Kanngiesser, N. Langhoff, R. Weddell, H. Wolf,

Springer, 2005, ISBN-10 3-540-28603-9

141. Portable X-Ray Fluorescence Spectrometry, Eds. P.J. Potts and M. West, RSC Publishing, 2008, ISBN 978-0-85404-552-5

142. ANew Method of Measuring Nuclear Magnetic Moment, I. Rabi, J.R. Zacharias, S. Millman, P. Kusch, Phys. Rev. 53 (1938) 318-318, http://dx.doi.org/10.1103/PhysRev.53.318

143. Mobile single-sided NMR, B. Blümich, J. Perlo, F. Casanova, Progress in Nuclear Magnetic Resonance Spectroscopy, 52 (2008) 197-269, http://dx.doi.org/10.1016/j.pnmrs.2007.10.002 
144. Noninvasive Testing of Art and Cultural Heritage by Mobile NMR,

B. Blümich, F. Casanova, J. Perlo, F. Presciutti, C. Anselmi, B. Doherty,

Accounts Chem. Res. 43 (2010) 761-770, http://dx.doi.org/10.1021/ar900277h

145. Mobile depth profiling and sub-surface imaging techniques for historical paintings - A review, M. Alfeld, J.A.C. Broekaert, Spectrochim Acta B. 88 (2013) 211-230, http://dx.doi.org/10.1016/j.sab.2013.07.009

146. NMR at low magnetic fields,

B. Blümich, F. Casanova,S. Appelt, Chem. Phys. Lett. 277 (2009) 231-240, http://dx.doi.org/10.1016/j.cplett.2009.06.096

147. The NMR MOUSE, a Mobile Universal Surface Explorer,

G. Eidmann, R. Savelsberg, P. Blümle, B. Blümich, J. Mag. Resonance, Series A 122 (1996) 104-109, http://dx.doi.org/10.1006/jmra.1996.0185

148. Compact NMR,

B. Blümich, S. Haber-Pholemeir, W. Zia,

De Greuyter (2014),

ISBN: 978-3-11-026671-9

149. Nuclear Magnetic Resonance to characterize and monitor Cultural Heritage,

D. Capitani, V. Di Tullio, N. Proietti,

Prog. Nuc. Mag. Res. Spectr. 64 (2012) 29-69,

http://dx.doi.org/10.1016/j.pnmrs.2011.11.001

150. Nondestructive Investigation of the Internal Structure of Fresco Paintings,

K. Fukunaga, T. Meldrum, W. Zia, M. Ohno, T. Fuchida, B. Blümich,

Proc. Digital Heritage (2013), pp. 81-88,

151. Modern Analytical Methods in Art and Archaeology, Eds. E. Cilibert, G. Spoto, Vol. 155 Chemical Analysis Series, John Willey \& Sons, 2000,

ISBN: 0-471-29361-X

152. Palazzo degli Alberti: le collezioni d'arte della Cariprato, CariPrato (in Italian),

Gruppo Banca Popolare di Vicenza, Ed. Skira, Milano (2004) pp.111-112, ISBN: 978-8884919922

153. X-ray Spectrometry (review),

K. Tsuji, K. Nakano, Y. Takahashi, K. Hayashi, Chul-Un Ro,

Anal. Chem., 84 (2012) 636-668,

http://dx.doi.org/10.1021/ac202871b
154. Analysis of Art objects Using a Portable X-ray Fluorescence Spectrometer,

P. Moioli, C. Seccaroni,

X-Ray Spectrom. 29 (2000) 48-52

http://dx.doi.org/10.1002/(SICl)1097-4539(200001/

02)29:1<48::AID-XRS404>3.0.CO;2-H

155. The differential PIXE set-up at the Van de Graaff laboratory in Florence,

M.E. Fedi, M. Chiari, L. Giuntini, F. Lucarelli, P.A. Mandò, Nucl. Instrum. Meth. Phys. Res. B 189 (2002) 56, http://dx.doi.org/10.1016/S0168-583X(01)00994-6

156. A novel portable XRF spectrometer with range of detection extended to low-Z elements,

A. Migliori, P. Bonanni, L. Carraresi, N. Grassi, P.A. Mandò, X-Ray Spectrom., 40 (2011) 107-112, http://dx.doi.org/10.1002/xrs.1316

157. New criterion for in situ, quick discrimination between traditionally maintained and artificially restored Japanese swords (katanas) by XRF spectrometry, L. Castelli, L. Giuntini, F. Taccetti, E. Barzagli, F. Civita, C. Czelusniak, M.E. Fedi, N. Gelli, A. Mazzinghi, L. Palla, F.P. Romano, P.A. Mandò,

X-Ray Spectrom., 42 (2013), 537-540,

http://dx.doi.org/10.1002/xrs.2516

158. XRF study on the gilding technique of the fresco 'Crocifissione con Santi' by Beato Angelico in the San Marco monastery in Florence,

A. Mazzinghi, L. Giuntini, N. Gelli, C. Ruberto,

X-Ray Spectrometry 44 (2015) 4,

http://dx.doi.org/10.1002/xrs.2650

159. XRF analyses for the study of paintings technique and degradation on frescoes by Beato Angelico: first results,

A. Mazzinghi,

II Nuovo Cimento C 37 (2014) 253-262, http://dx.doi.org/10.1393/ncc/i2014-11809-0

160. Earth pigments in painting: characterisation and differentiation by means of FTIR spectroscopy and SEM-EDS microanalysis,

C. Genestar, C. Pons,

Anal. Bioanal. Chem. 382 (2005) 269-274,

http://dx.doi.org/10.1007/s00216-005-3085-8

161. Ochre-differentiation through micro-Raman and micro-FTIR spectroscopies: application on wall paintings at Meteora and Mount Athos, Greece,

D. Bikiaris, Sister Daniilia, S. Sotiropoulou, O. Katsimbiri,

E. Pavlidou, A.P. Moutsatsou, Y. Chryssoulakis,

Spectrochimica Acta A 56 (2000) 3-18,

http://dx.doi.org/10.1016/S1386-1425(99)00134-1 
162. Diagnostics in Arts and Culture,

H. Oliver, in Handbook of Technical Diagnostics, Ed. H. Czichos, Springer, 2013, pp. 545-556, ISBN 978-3-642-25850-3

163. Non-destructive analysis and testing of museum objects: An overview of 5 years of research,

A. Adriaens,

Spectrochimica Acta B 60 (2005) 1503-1516, http://dx.doi.org/10.1016/j.sab.2005.10.006

164. Non-invasive studies of objects from cultural Heritage, E.H. Lehmann, P. Vontobel, E. Deschler-Erb, M. Soares, Nucl. Instrum. Meth. Phys. Res. A 542 (2005) 68-75, http://dx.doi.org/10.1016/j.nima.2005.01.013

165. Advantages of scanning-mode ion beam analysis for the study of Cultural Heritage,

N. Grassi, L. Giuntini, P.A. Mandò, M. Massi,

Nucl. Instrum. Meth. Phys. Res. B 256 (2007) 712-718, http://dx.doi.org/10.1016/j.nimb.2006.12.196

166. Micro-Raman and X-ray fluorescence spectroscopy data fusion for the classification of ochre pigments, P.M. Ramos, I. Ruisánchez, K.S. Andrikopoulos, Talanta 75 (2008) 926-936, http://dx.doi.org/10.1016/j.talanta.2007.12.030

167. Identification of copper-based green pigments in Jaume Huguet's Gothic altarpieces by Fourier transform infrared microspectroscopy and synchrotron radiation $X$-ray diffraction,

N. Salvadó, T. Pradell, E. Pantos, M.Z. Papiz, J. Molera, M. Seco, M. Vendrell-Saz,

J. Synchrotron Rad. 9 (2002) 215-222, http://dx.doi.org/10.1107/S0909049502007859

168. Lead White,

R.J. Gettens, H. Kühn, W.T. Chase,

Studies in conservation 12 (1967) 125-139,

http://dx.doi.org/10.1179/sic.1967.013
169. La chimica nel restauro, I Materiali dell'arte pittorica, M. Matteini, A. Moles, Nardini, Florence, 2007, ISBN 9788840441542

170. Historical pigments: a collection analyzed with X-ray diffraction analysis and $X$-ray fluorescence analysis in order to create a database,

B. Hochleitner, V. Desnica, M. Mantler, M. Schreiner, Spectrochimica Acta Part B 58 (2003) 641-649, http://dx.doi.org/10.1016/S0584-8547(02)00280-X

171. Raffaello, "La Muta" - Indagini e restauro, M. Ciatti, M.R. Valazzi, (Problemi di conservazione e restauro 43), Florence, 2015, ISBN 88-7970-736-1

172. Vermilion and Cinnabar,

R.J. Gettens, R.L. Feller, W.T. Chase,

Studies in Conservation 17 (1972) 45-69,

http://dx.doi.org/10.1179/sic.1972.006

173. Multispectral Infrared Reflectography to Differentiate Features in Paintings,

C. Daffara, R. Fontana,

Microsc. Microanal. 17 (2011) 691-695,

http://dx.doi.org/10.1017/S1431927611000031

174. I principi scientifici del Divisionismo. (La tecnica della pittura),

G. Previati,

Torino Bocca 1906.

175. Neo-Impressionism,

R. L. Herbert,

The Solomon R. Guggenheim Foundation, New York, 1968.

176. Non-destructive XRF analysis of paintings,

Z. Szőkefalvi-Nagy, I. Demeter, A. Kocsonya, I. Kovács, Nucl. Instrum. Meth. Phys. Res. B 226 (2004) 53-59, http://dx.doi.org/10.1016/j.nimb.2004.03.074

\section{CHAPTER 6. PRESERVATION OF CULTURAL HERITAGE}

177. Sauvé des eaux - Le patrimoine archéologique en bois, histoires de fouilles et de restaurations, $H$. Bernard-Maugiron, P. Coeuré, M. Clermont-Joly, P. Veysseyre,

ARCNucléart, 2007, IBSN: 978-2-9529035-0-9

178. Procédés radiochimiques pour la conservation des biens culturels, P. Coeuré, Q.K. Tran,

Actualite Chimique, 11 (1999) 141-143.
179. Nuclear Techniques for Preservation of Cultural Heritage Artefacts (brochure),

L. Cortella, Q.K. Tran, W.J. Głuszewski, I.V. Moise, C.C. Ponta,

IAEA Technical Cooperation Project - RER 8015: Using Nuclear Techniques for the Characterization and Preservation of Cultural Heritage Artefacts in the European Region, 2011.

180. Maria Skłodowska Curie-the precursor of radiation sterilization methods, 
W. Głuszewski, Z.P. Zagórski, Q.K. Tran, Q. Khoï, L. Cortella,

Anal. Bioanal. Chem. 400 (2011) 1577-1582, http://dx.doi.org/10.1007/s00216-011-4699-7

181. Establishing the irradiation dose for paper decontamination,

I.V. Moise, M. Virgolici, C.D. Negut, M. Manea, M. Alexandru, L. Trandafir, F.L. Zorila, C.M. Talasman, D. Manea, S. Nisipeanu, M. Haiducu, Z. Balan,

Rad. Phys. Chem. 81 (2012) 1045-1050,

http://dx.doi.org/10.1016/j.radphyschem.2011. 11.063

182. Gamma radiation for preservation of biologically damaged paper,

M.E. Gonzalez, A.M. Calvo, E. Kairiyama,

Rad. Phys. Chem. 63 (2002) 263-265,

http://dx.doi.org/10.1016/S0969-806X(01)00510-2

183. The recovery of biodeteriorated books and archive documents through gamma radiation: Some considerations on the results achieved,

G. Magaudda,

J. Cult. Heritage 5 (2004) 113-118,

http://dx.doi.org/10.1016/j.culher.2003.07.003

184. Gamma radiation effects on physical properties of parchment documents: Assessment of Dmax,
I. Nunes, N. Mesquita, S.C. Verde, M.J. Trigo, A. Ferreira, M.M. Carolino, A. Portugal, M.L. Botelho,

Rad. Phys. Chem. 81 (2012) 1943-1946,

http://dx.doi.org/10.1016/j.radphyschem.2012.07.016

185. ARC-Nucléart, http://www.arc-nucleart.fr

186. Nucleart: Nuclear techniques applied to art, R.Ramiere, Q.K. Tran, Nucl. Europe 7 (1989) 50

187. La désinfection des biens culturels par irradiation gamma,

R. Ramière,

in Les contaminants biologiques des biens culturels, Ed. M.F. Roquebert, 2002, Elsevier, Paris, 291-293.

188. Impregnation with radiation-curing monomers and resins,

Q.K. Tran, R. Ramiere, A. Giniergillet,

Advances in Chemistry series, 225 (1990) 217-233, http://dx.doi.org/10.1021/ba-1990-0225.ch009 ISBN13: 9780841216235

189. Irradiation Conservation of Cultural Heritage, C.C. Ponta

Nucl Phys News 18 (2008), 22-24, http://dx.doi.org/10.1080/10506890801927213

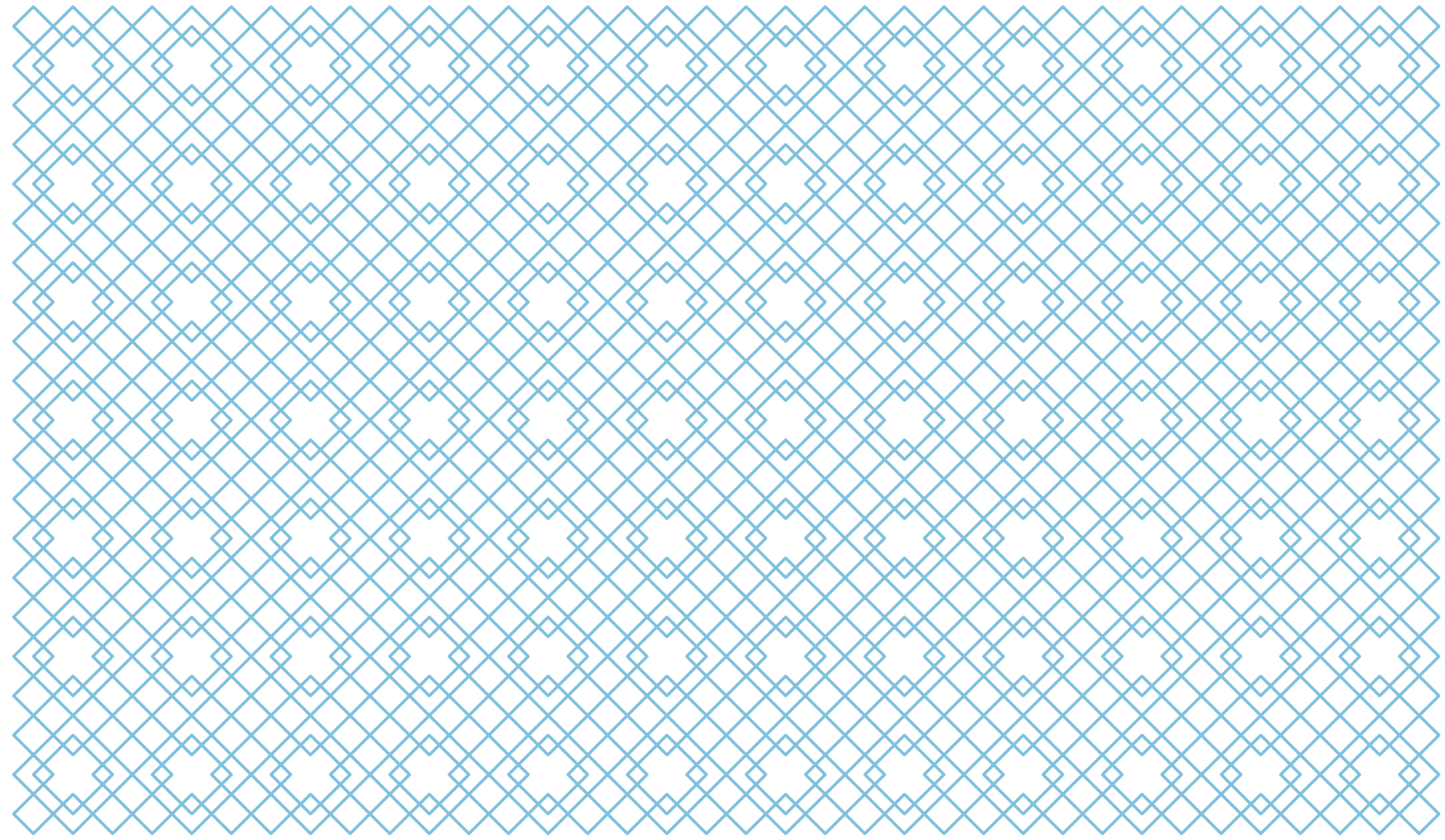




\section{AE \\ Nuclear \\ Physics \\ 世}

ADDRESS

6, RUE DES FRÈRES LUMIÈRE 68200 MULHOUSE - FRANCE

\section{CONTACT}

WEBSITE: WWW.EPS.ORG

PHONE: $\quad+33389329440$

FAX: $\quad+33389329449$

This is an open access article

under the CC BY-NC-ND license

(https://creativecommons.org/licenses/

by-nc-nd/4.0/).

DOI: $10.1071 / 978-2-7598-2091-7$

ISBN: 978-2-7598-2091-7

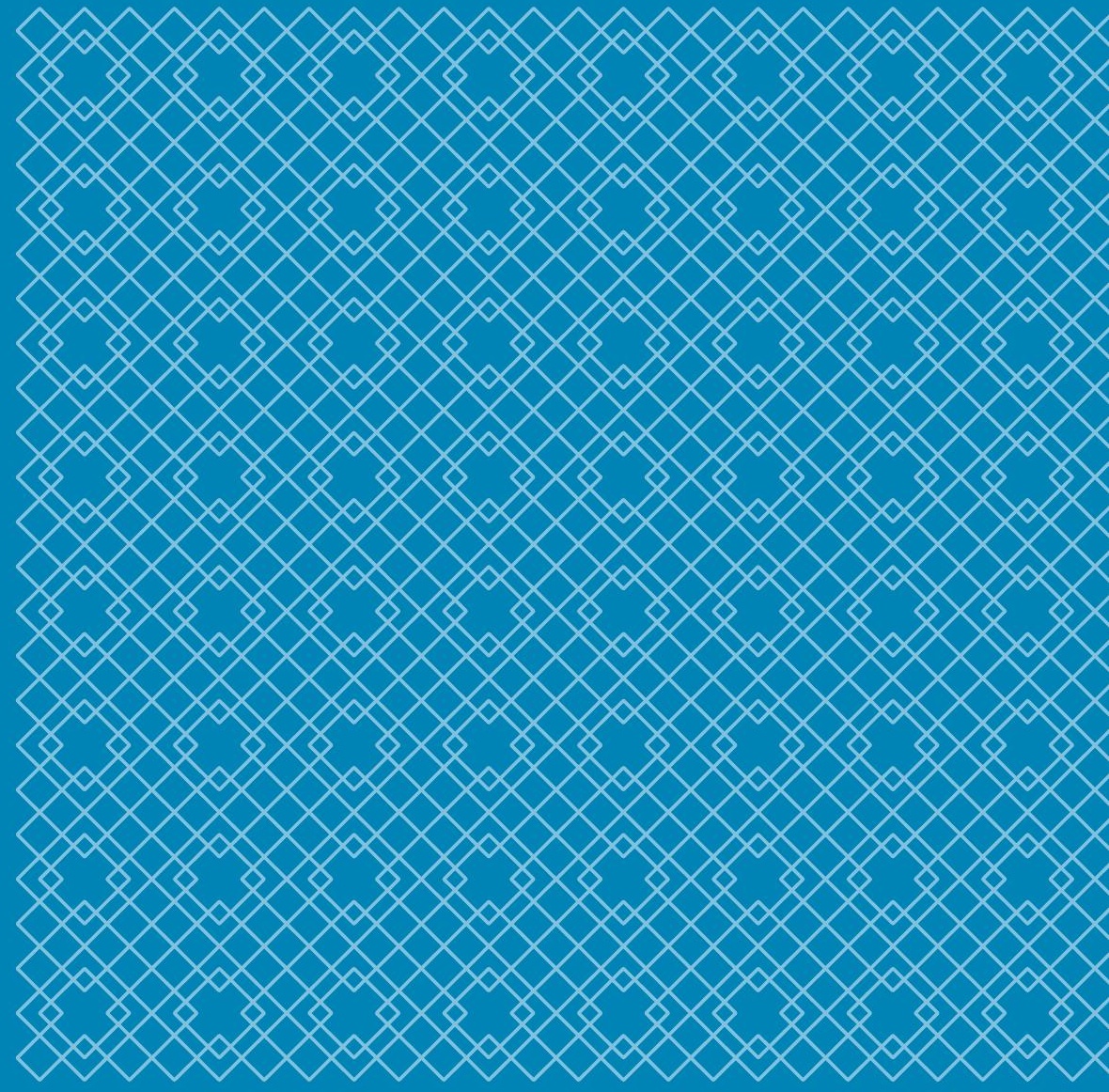

